\title{
LOCAL MODELS OF SHIMURA VARIETIES AND A CONJECTURE OF KOTTWITZ
}

\author{
G. PAPPAS AND X. ZHU
}

\begin{abstract}
We give a group theoretic definition of "local models" as sought after in the theory of Shimura varieties. These are projective schemes over the integers of a $p$-adic local field that are expected to model the singularities of integral models of Shimura varieties with parahoric level structure. Our local models are certain mixed characteristic degenerations of Grassmannian varieties; they are obtained by extending constructions of Beilinson, Drinfeld, Gaitsgory and the second-named author to mixed characteristics and to the case of general (tamely ramified) reductive groups. We study the singularities of local models and hence also of the corresponding integral models of Shimura varieties. In particular, we study the monodromy (inertia) action and show a commutativity property for the sheaves of nearby cycles. As a result, we prove a conjecture of Kottwitz which asserts that the semi-simple trace of Frobenius on the nearby cycles gives a function which is central in the parahoric Hecke algebra.
\end{abstract}

Key words: Shimura variety, affine flag variety, local model, group scheme, nearby cycles.

\section{Contents}

Introduction

$\S 1$. Preliminaries

$\S 2$. Reductive groups over $\mathcal{O}\left[u, u^{-1}\right]$

$\S 3$. Parahoric group schemes over $\mathcal{O}[u]$

$\S 4$. Classical groups

$\S 5$. Loop groups and affine Grassmannians

$\S 6$. Local models

$\S 7$. Shimura varieties and local models

$\S 8$. The special fibers of the local models

$\S 9$. Nearby cycles and the conjecture of Kottwitz

$\S 10$. Appendix: Homogeneous spaces References

Date: November 27, 2012.

G. P partially supported by NSF grant DMS11-02208.

X. Z partially supported by NSF grant DMS10-01280. 


\section{INTRODUCTION}

The "local models" of this paper are projective schemes over the integers of a $p$-adic local field that are expected to model the singularities of integral models of Shimura varieties at places of (tame, parahoric) bad reduction. This is meant in the sense that each point on the integral model of the Shimura variety should have an étale neighborhood which is isomorphic to an étale neighborhood of a corresponding point on the local model. The simplest example is for the classical modular curve $X_{0}(p)$ with $\Gamma_{0}(p)$-level structure. In this case, the local model is obtained by blowing up the projective line $\mathbb{P}_{\mathbb{Z}_{p}}^{1}$ over $\operatorname{Spec}\left(\mathbb{Z}_{p}\right)$ at the origin 0 of the special fiber $\mathbb{P}_{\mathbb{F}_{p}}^{1}$. More generally, local models for Shimura varieties of PEL type with parahoric level structure were given by Rapoport and Zink in [55]. Their construction was tied to the description of the Shimura variety as a moduli space of abelian schemes with additional structures; the fact that they capture the singularities of this moduli space is a consequence of the Grothendieck-Messing deformation theory of abelian schemes. However, it was soon realized that the Rapoport-Zink construction is not adequate when the group of the Shimura variety is ramified at $p$ and in many cases of orthogonal groups. Indeed, then the corresponding integral models are often not flat ([46]). In the ramified PEL case, corrected integral models were considered and studied in [46], 47], 48], [50]. These are flat by definition but they do not always have a neat moduli space interpretation. Unfortunately, the constructions in these works are somewhat ad-hoc and are mostly done case-by-case: Ultimately, they are based on representing the corresponding reductive group explicitly as the neutral component of the group of automorphisms of a suitable bilinear (symmetric, alternating, hermitian etc.) form on a vector space over a division ring. Then, its parahoric subgroups are the connected stabilizers of a self-dual chain of lattices as explained in [8], [10] (see also [55, Appendix to Ch. 3]) and the local models are given as flat closures in certain corresponding linked Grassmannians. We refer the reader to the survey [51] for precise definitions and more references.

In this paper, we provide a general group theoretic definition of local models that is not tied to a particular representation of the group. This approach allows us to make significant progress and resolve several open questions. Our local models are constructed starting from the "local Shimura data", i.e triples $(G, K,\{\mu\})$, where $G$ is a (connected) reductive group over $\mathbb{Q}_{p}, K \subset G\left(\mathbb{Q}_{p}\right)$ a parahoric "level" subgroup, and $\{\mu\}$ a geometric conjugacy class of one parameter subgroups $\mu: \mathbb{G}_{\mathrm{m}} / \overline{\mathbb{Q}}_{p} \rightarrow G_{\overline{\mathbb{Q}}_{p}}$ over an algebraic closure $\overline{\mathbb{Q}}_{p}$. Here, we assume that $\mu$ is minuscule. Denote by $E$ the field of definition of the conjugacy class $\{\mu\}$. This is the local reflex field; it is a finite extension of $\mathbb{Q}_{p}$ and is contained in a splitting field for $G$. We will assume that $G$ splits over a tamely ramified extension of $\mathbb{Q}_{p}$. Let $\mathcal{O}_{E}$ be the ring of integers of $E$ and $k_{E}$ its residue field. By definition, the local model $\mathrm{M}^{\text {loc }}$ is a projective scheme over $\operatorname{Spec}\left(\mathcal{O}_{E}\right)$ with generic fiber the homogeneous space for $G_{E}$ that corresponds to $\mu$. Our first main result is the following (a weaker version of a combination of Theorems 8.1 and 8.3):

\footnotetext{
${ }^{1}$ This is an important assumption that we keep throughout the paper.
} 
Theorem 0.1. Suppose that the prime $p$ does not divide the order of the fundamental group $\pi_{1}\left(G\left(\overline{\mathbb{Q}}_{p}\right)_{\text {der }}\right)$. Then $\mathrm{M}^{\text {loc }}$ is normal with reduced special fiber; all the irreducible components of the geometric special fiber $\mathrm{M}^{\mathrm{loc}} \otimes_{\mathcal{O}_{E}} \overline{\mathbb{F}}_{p}$ are normal and Cohen-Macaulay. In fact, $\mathrm{M}^{\mathrm{loc}} \otimes_{\mathcal{O}_{E}} \overline{\mathbb{F}}_{p}$ can be identified with the reduced union of a finite set of affine Schubert varieties in the affine flag variety $\mathrm{Gr}_{\mathcal{G}, \mathbb{F}_{p}} \otimes_{\mathbb{F}_{p}} \overline{\mathbb{F}}_{p}$; the set parametrizing this union is the " $\mu$-admissible set" defined by Kottwitz and Rapoport.

The definition of $\mathrm{M}^{\mathrm{loc}}$ and of the affine flag variety $\mathrm{Gr}_{\mathcal{G}, \mathbb{F}_{p}} \otimes_{\mathbb{F}_{p}} \overline{\mathbb{F}}_{p}$ will be explained below; the rest is given in 8, . The main ingredients in the proof of this theorem are results of [16, 49] on the structure of Schubert varieties in affine flag varieties and the coherence conjecture of [49] which was shown by the second-named author in [76].

In the case of Shimura data of PEL type, we show that the local models $\mathrm{M}^{\text {loc }}$ agree with the "corrected local models" which are obtained (in most cases at least) by taking the flat closures of the "naive local models" of Rapoport-Zink; these corrected local models do describe the étale local structure of corresponding integral models of Shimura varieties as we discussed in the beginning of the introduction. For PEL types the result in the above theorem was conjectured and verified for a few special cases in several papers ([19], [20], [47], [48], [50], 49], see also [51]). In [76], the theorem is proven for the ramified unitary similitude groups. Our approach here allows a unified treatment in almost all cases.

For example, let us explain a result that follows by combining the above with the work of Rapoport and Zink. Suppose that $\mathfrak{D}=\left(\mathbf{B}, \mathcal{O}_{\mathbf{B}},{ }^{*}, \mathbf{V},(),, \mu,\{\mathcal{L}\}, K^{p}\right)$ give PEL data as in $97 . \mathrm{b}$, 7 .b.4 with corresponding group $\mathbf{G}$ over $\mathbb{Q}$ and reflex field $\mathbf{E}$. Assume that $p$ is odd, that $K^{p} \subset \mathbf{G}\left(\mathbb{A}_{f}^{p}\right)$ is sufficiently small and that the subgroup $K=K_{p}$ of $\mathbf{G}\left(\mathbb{Q}_{p}\right)$ that stabilizes the lattice chain $\{\mathcal{L}\}$ is parahoric. Suppose that $G=\mathbf{G}_{\mathbb{Q}_{p}}$ splits over a tamely ramified extension of $\mathbb{Q}_{p}$ and, in addition, that $\mathbf{G}$ is connected. Let $\mathfrak{P}$ be a prime of $\mathbf{E}$ over $p$. Under these assumptions, we obtain:

Theorem 0.2. The Shimura variety $S h_{\mathbf{K}}$ defined by the PEL data $\mathfrak{D}$ affords a flat integral model $\mathcal{S}_{\mathbf{K}}$ over $\mathcal{O}_{\mathbf{E}_{\mathfrak{P}}}$ which is, locally for the étale topology, isomorphic to the local model $\mathrm{M}^{\mathrm{loc}}$ for $(G, K,\{\mu\})$. The scheme $\mathcal{S}_{\mathbf{K}}$ is normal with reduced special fiber; the geometric special fiber $\mathcal{S}_{\mathbf{K}} \otimes_{\mathcal{O}_{E}} \overline{\mathbb{F}}_{p}$ admits a stratification with locally closed strata parametrized by the $\mu$-admissible set; the closure of each stratum is normal and Cohen-Macaulay.

In fact, the result is more precise: We show the existence of a "local model diagram" (see (7.1) ); also, the model $\mathcal{S}_{\mathbf{K}}$ is the flat closure of the generic fiber of the corresponding Rapoport-Zink integral model and thus supports a natural morphism to a Siegel moduli scheme. See $\$ 7 . b .5$ for the proof and for more similar results, in particular for a discussion of cases in which $\mathbf{G}$ is not connected. (Note that, in general, $S h_{\mathbf{K}}$ above is equal to a disjoint union of Shimura varieties in the sense of Deligne; this is related to the failure of the Hasse principle, see [36, §8] and Remark 7.3) In general, we conjecture that the general Shimura variety with local data $(G, K,\{\mu\})$ has an integral model which affords a local model diagram and hence is locally for the étale topology isomorphic to $\mathrm{M}^{\mathrm{loc}}$. Showing this, in cases of Shimura varieties of abelian type, is the subject of joint work in preparation of the first author with M. Kisin [31]. This combined with Theorem 0.1 will then imply that the conclusion of Theorem 0.2 also holds for such Shimura varieties. 
Before considering Kottwitz's conjecture, we will explain our definition of local models. This uses the construction of certain group schemes over a two-dimensional base and of their corresponding (affine) flag varieties. We believe that these objects are of independent interest and we begin by discussing them in some detail.

We start by discussing the group schemes. Let $F$ be a $p$-adic field with ring of integers $\mathcal{O}$ and residue field $k$. Suppose $G$ is a reductive group over $F, K$ a parahoric subgroup of $G(F)$. By definition, $K$ is the (connected) stabilizer of a point $x$ in the Bruhat-Tits building $\mathcal{B}(G, F)$ of $G(F)$. In [7], Bruhat-Tits construct a smooth group scheme $\mathcal{P}_{x}$ over the discrete valuation ring $\mathcal{O}$ such that $K=\mathcal{P}_{x}(\mathcal{O})$. Assume that $G$ splits over a tamely ramified extension of $F$. Choose a uniformizer $\varpi$ of $\mathcal{O}$. In the first part of the paper, we construct a smooth affine group scheme $\mathcal{G}$ over the affine line $\mathbb{A}_{\mathcal{O}}^{1}=\operatorname{Spec}(\mathcal{O}[u])$ which has connected fibers, is reductive over the complement of $u=0$ and specializes to $\mathcal{P}_{x}$ along the section $\operatorname{Spec}(\mathcal{O}) \rightarrow \mathbb{A}_{\mathcal{O}}^{1}$ given by $u \mapsto \varpi$. In addition, the base changes of $\mathcal{G}$ by $\mathcal{O}[u] \rightarrow F[[u]]$ and $\mathcal{O}[u] \rightarrow k[[u]]$ give corresponding Bruhat-Tits group schemes over these two discrete valuation rings.

Having given $\mathcal{G}$, we can now define various mixed characteristic versions of the familiar (from the theory of geometric Langlands correspondence) global and local versions of the affine Grasmannian and the affine flag variety. For simplicity, we set $X=\mathbb{A}_{\mathcal{O}}^{1}=$ $\operatorname{Spec}(\mathcal{O}[u])$. The main actor is the global affine Grassmannian $\operatorname{Gr}_{\mathcal{G}, X}$; this is the moduli functor on schemes over $X$ which to $y: S \rightarrow X$ associates the set of isomorphism classes of $\mathcal{G}$-bundles over $X \times_{\mathcal{O}} S$ together with a trivialization on the complement of the graph of $y$. When $\mathcal{O}$ is replaced by a field and $\mathcal{G}$ is a constant split reductive group, this is the global affine Grassmannian (over the affine line) of Beilinson and Drinfeld. We show that $\mathrm{Gr}_{\mathcal{G}, X}$ is represented by an ind-scheme which is ind-proper over $X$. Denote by $\operatorname{Gr}_{\mathcal{G}, \mathcal{O}} \rightarrow \operatorname{Spec}(\mathcal{O})$ the base change of $\operatorname{Gr}_{\mathcal{G}, X} \rightarrow X$ by $\operatorname{Spec}(\mathcal{O}) \rightarrow X$ given by $u \mapsto \varpi$. We can easily see, using the descent lemma of Beauville and Laszlo, that the generic fiber $\operatorname{Gr}_{\mathcal{G}, \mathcal{O}} \otimes_{\mathcal{O}} F$ is isomorphic to the affine Grassmannian $\operatorname{Gr}_{G, F}$ for the loop group $G \otimes_{F} F((t)), t=u-\varpi$. Recall that $\operatorname{Gr}_{G, F}$ represents the fpqc sheaf given by $R \mapsto G(R((t))) / G(R[[t]])$. Similarly, the special fiber $\operatorname{Gr}_{\mathcal{G}, \mathcal{O}} \otimes_{\mathcal{O}} k$ is isomorphic to an affine flag variety $\operatorname{Gr}_{\mathcal{G}, k}$ for the group $\mathcal{G}(k((u)))$ over the local field $k((u))$ and its parahoric subgroup $\mathcal{G}(k[[u]])$ 2 3 Here $\operatorname{Gr}_{\mathcal{G}, k}$ represents $R \mapsto \mathcal{G}(R((u))) / \mathcal{G}(R[[u]])$.

We are now ready to give our definition of the local model $\mathrm{M}^{\mathrm{loc}}$. For this we take $F=\mathbb{Q}_{p}$ in the above and use the "local Shimura data" $(G, K,\{\mu\})$ with local reflex field $E$. As above, we need to assume that $G$ splits over a tamely ramified extension of $\mathbb{Q}_{p}$. Since $\mathbb{G}_{\mathrm{m}}=\operatorname{Spec}\left(\mathbb{Q}_{p}\left[t, t^{-1}\right]\right)$, the coweight $\mu$ provides a $\overline{\mathbb{Q}}_{p}((t))$-valued point $s_{\mu}$ of $G$ and hence a $\overline{\mathbb{Q}}_{p}$-valued point $\left[s_{\mu}\right]$ of $\operatorname{Gr}_{G, \mathbb{Q}_{p}}$. Because $\mu$ is minuscule, the (left) $G\left(\overline{\mathbb{Q}}_{p}[[t]]\right)$ orbit of $\left[s_{\mu}\right]$ is a smooth projective variety $X_{\mu}$ (actually a homogeneous space for $G_{E}$ ) defined over the local reflex field $E ; X_{\mu}$ is a closed subvariety of the affine Grassmannian $\operatorname{Gr}_{G, \mathbb{Q}_{p}} \otimes_{\mathbb{Q}_{p}} E=\operatorname{Gr}_{\mathcal{G}, \mathcal{O}} \otimes_{\mathcal{O}} E$. By definition, the local model $\mathrm{M}^{\mathrm{loc}}:=M_{\mathcal{G}, \mu}$ is the reduced

\footnotetext{
${ }^{2}$ In [49, affine flag/Grassmannian varieties for groups that are not necessarily constant are referred to as "twisted". Here we omit this adjective.
} 
projective scheme over $\operatorname{Spec}\left(\mathcal{O}_{E}\right)$ given by the Zariski closure of $X_{\mu} \subset \operatorname{Gr}_{\mathcal{G}, \mathcal{O}} \otimes_{\mathcal{O}} E$ in the ind-scheme $\operatorname{Gr}_{\mathcal{G}, \mathcal{O}} \otimes_{\mathcal{O}} \mathcal{O}_{E}$.

By construction, the special fiber $\mathrm{M}^{\mathrm{loc}} \otimes_{\mathcal{O}_{E}} k_{E}$ is a closed subscheme of the affine flag variety $\operatorname{Gr}_{\mathcal{G}, \mathbb{F}_{p}} \otimes_{\mathbb{F}_{p}} k_{E}$. Let us remark here that the local models $\mathrm{M}^{\text {loc }}$ are given by taking a Zariski closure and, as a result, they do not always have a neat moduli space interpretation. This issue does not concern us in this paper. Indeed, the close relation of $\mathrm{M}^{\text {loc }}$ to the affine Grassmannians allows us to show directly many favorable properties as in Theorem 0.1. These then imply nice properties of corresponding integral models for Shimura varieties as in Theorem 0.2 .

The same connection with the theory of affine Grassmannians also allows us to obtain results about the sheaf of nearby cycles $\mathrm{R} \Psi\left(\overline{\mathbb{Q}}_{\ell}\right)$ of the scheme $\mathrm{M}^{\text {loc }} \rightarrow \operatorname{Spec}\left(\mathcal{O}_{E}\right)$. (Here $\ell$ is a prime different from $p$.) We will describe these below. Recall that we conjecture that $\mathrm{M}^{\mathrm{loc}}$ describes the étale local structure of an integral model of the Shimura variety. Therefore, the nearby cycles of the local models should also be determining the nearby cycles for integral models of Shimura varieties with parahoric level structure. (As follows from the above, this is indeed the case for most PEL types.) Our results will be useful in expressing the local factor of the Hasse-Weil zeta function of the Shimura variety at places of (tame) parahoric reduction as a product of local factors of automorphic L-functions. The strategy of using the local model to determine the (semi-simple) HasseWeil zeta function was first suggested by Rapoport [54. It has since been advanced by Kottwitz, Haines-Ngô ([25]), Haines and others. A centerpiece of this approach is a conjecture of Kottwitz that states that, in the case that $G$ is split, the semi-simple trace of Frobenius on the sheaf of nearby cycles gives a central function in the parahoric Hecke algebra. This was proven by Haines and Ngô ([25]) in (split) types A and C by following an argument of Gaitsgory [17. Gaitsgory proved a stronger statement for general split groups in the function field case; he showed that the perverse sheaf of nearby cycles satisfies a commutativity constraint with respect to the convolution product. This implies Kottwitz's conjecture in the function field case. The main tools in Gaitsgory's approach are various versions of the global affine Grassmannian of Beilinson-Drinfeld. In this paper, we are able to generalize and simplify the approaches of both Gaitsgory and Haines-Ngô by using the mixed characteristic affine Grassmannians $\operatorname{Gr}_{\mathcal{G}, X}$ and various other related ind-schemes. In particular, we obtain a general result even for non-split groups as follows:

Our construction of the group scheme $\mathcal{G}$ over $X=\operatorname{Spec}\left(\mathbb{Z}_{p}[u]\right)$ also provides us with a reductive group $G^{\prime}=\mathcal{G} \times{ }_{X} \operatorname{Spec}\left(\mathbb{F}_{p}((u))\right)$ over $\mathbb{F}_{p}((u))$ and a parahoric subgroup $K^{\prime}$ which correspond to $G$ and $K$ respectively. By definition, if $\mathbb{F}_{q} \supset k_{E}$, we have an equivariant embedding $\mathrm{M}^{\mathrm{loc}} \otimes_{\mathcal{O}_{E}} \mathbb{F}_{q} \subset \mathrm{Gr}_{\mathcal{G}, \mathbb{F}_{p}} \otimes_{\mathbb{F}_{p}} \mathbb{F}_{q}=\operatorname{Gr}_{G^{\prime}, \mathbb{F}_{p}} \otimes_{\mathbb{F}_{p}} \mathbb{F}_{q}$. This allows us to view the Frobenius trace function $\tau_{\mathrm{R} \Psi}^{\mathrm{sS}}(x)=\operatorname{tr}^{\mathrm{SS}}\left(\operatorname{Frob}_{x}, \mathrm{R} \Psi\left(\overline{\mathbb{Q}}_{\ell}\right)_{\bar{x}}\right), x \in \mathrm{M}^{\mathrm{loc}}\left(\mathbb{F}_{q}\right)$, as an element of the parahoric Hecke algebra $\mathcal{H}_{q}\left(G^{\prime}, K^{\prime}\right)$ of bi- $K^{\prime}\left(\mathbb{F}_{q}[[u]]\right)$-invariant, compactly supported locally constant $\overline{\mathbb{Q}}_{\ell}$-valued functions on $G^{\prime}\left(\mathbb{F}_{q}((u))\right)$. 
Theorem 0.3. (Kottwitz's conjecture) The semi-simple trace of Frobenius on the sheaf of nearby cycles $\mathrm{R} \Psi\left(\overline{\mathbb{Q}}_{\ell}\right)$ of $\mathrm{M}^{\mathrm{loc}} \rightarrow \operatorname{Spec}\left(\mathcal{O}_{E}\right)$ gives a central function $\tau_{\mathrm{R} \Psi}^{\mathrm{ss}}$ in the parahoric Hecke algebra $\mathcal{H}_{q}\left(G^{\prime}, K^{\prime}\right)$.

See $99 . \mathrm{d}$ for more details and in particular Theorem 9.14 for the precise statement which is more general. In the split Iwahori case, as a corollary of this theorem, one can give an explicit formula for the semi-simple Frobenius trace function using Bernstein's presentation of the Iwahori-Hecke algebra, as was explained in [25]. In fact, more generally, when $G$ is unramified and the subgroup an arbitrary parahoric, we show that the semi-simple trace can be expressed as a Bernstein function in the parahoric Hecke algebra as was also conjectured by Kottwitz and by Haines. Let us mention here that Kottwitz's conjecture for quasi-split (but not split) unramified unitary groups was also shown independently by S. Rostami in his thesis [59].

We also obtain results for quasi-split ramified groups when the level subgroup is very special. Here and elsewhere, we say that $K \subset G\left(\mathbb{Q}_{p}\right)$ is very special, if the corresponding parahoric subgroup of $G\left(\mathbb{Q}_{p}^{\text {unr }}\right)$ is special in the sense of Bruhat-Tits; then $K$ itself is also special. In this case, we give a characterization of the function $\tau_{\mathrm{R} \Psi}^{\mathrm{ss}}$ by identifying its trace on unramified representations; this also agrees with the prediction of a conjecture of Haines and Kottwitz. Corresponding to $\mu$ we have a minuscule coweight for $G^{\prime}$ which for simplicity we still denote by $\mu$. The conjugacy class $\{\mu\}$ defines an algebraic $\overline{\mathbb{Q}}_{\ell^{-}}$ representation $V_{\mu}$ of the Langlands dual group ${ }^{L} G^{\prime}=H^{\vee} \rtimes \operatorname{Gal}_{\mathbb{F}_{q}((u))}$ of $G^{\prime}$ over $\mathbb{F}_{q}((u))$. (Here $H$ is the Chevalley split form of $G^{\prime}$ ). The inertia invariants $V_{\mu}^{I}$ give a representation of $\left(H^{\vee}\right)^{I} \rtimes \operatorname{Gal}\left(\overline{\mathbb{F}}_{q} / \mathbb{F}_{q}\right)$. If $\pi$ is an irreducible smooth representation of $G^{\prime}\left(\mathbb{F}_{q}((u))\right)$ with a $K^{\prime}\left(\mathbb{F}_{q}[[u]]\right)$-fixed vector, one can define its Langlands-Satake parameter $\operatorname{Sat}(\pi): W \rightarrow$ ${ }^{L} G^{\prime}$ : Among other properties, one can show that if $\Phi_{q} \in W$ is a (geometric) Frobenius element in the Weil group, then $\operatorname{Sat}(\pi)\left(\Phi_{q}\right)$ is a semi-simple element in $\left(H^{\vee}\right)^{I} \times$ Frob $_{q}^{-1}$, which is well-defined up to $\left(H^{\vee}\right)^{I}$-conjugacy and completely determines $\operatorname{Sat}(\pi)$. Our characterization of the Frobenius trace function is the identity

$$
\operatorname{tr}\left(\pi\left(\tau_{\mathrm{R} \Psi}^{\mathrm{ss}}\right)\right)=\operatorname{tr}\left(\operatorname{Sat}(\pi)\left(\Phi_{q}\right), V_{\mu}^{I}\right)
$$

for all $\pi$ as above. This is shown by combining our constructions with the results in [75], [58. See the last section of the paper for more details.

As we mentioned above, when the Shimura data are of PEL type, the local model does indeed describe the étale local structure of an integral model of the Shimura variety. Therefore, in this case, our results also give the semi-simple trace of Frobenius on the nearby cycles of an integral model of the Shimura variety. One can then apply them to the calculation of the local factor of the Hasse-Weil zeta function of the Shimura variety following the arguments of Kottwitz, Rapoport and Haines (e.g [54, 23]), but we will not go into this here. (We also expect that this approach will be extended to many Shimura varieties with parahoric level which are not of PEL type using [31.) Here we should also mention very recent results of Scholze [60] and Scholze and Shin 61] that make progress towards this calculation without having to explicitly identify the semi-simple trace. 
Finally, we give several results about the action of monodromy (i.e of inertia) on the sheaves of nearby cycles. These also imply corresponding results for Shimura varieties. Here is an example:

Theorem 0.4. Assume that $G$ is split over the (tamely ramified) extension $F / \mathbb{Q}_{p}$ and that $K$ is a very special subgroup of $G\left(\mathbb{Q}_{p}\right)$. Then the inertia subgroup $I_{F}=\operatorname{Gal}\left(\overline{\mathbb{Q}}_{p} / \mathbb{Q}_{p}^{\text {unr }} F\right)$ acts trivially on the sheaf of nearby cycles $\mathrm{R} \Psi\left(\overline{\mathbb{Q}}_{\ell}\right)$ of $\mathrm{M}^{\mathrm{loc}} \rightarrow \operatorname{Spec}\left(\mathcal{O}_{E}\right)$.

More generally, without the assumption that $K$ is very special, we show that the action of $I_{F}$ on the sheaf of nearby cycles $\mathrm{R} \Psi\left(\overline{\mathbb{Q}}_{\ell}\right)$ is unipotent. In this case, we can describe the action of the full inertia group $I_{E}$ in terms of the geometric Satake equivalence for ramified groups of [75]. This is obtained by comparing nearby cycles along two directions over the two-dimensional base $\mathcal{O}[u]$ and is used in proving the identity (0.1) above. In the case that $G=\operatorname{Res}_{F / \mathbb{Q}_{p}} \mathrm{GL}_{n}$ with $F / \mathbb{Q}_{p}$ tame, this description of the inertia action confirms a conjecture in [47.

Recall that, throughout the paper, we have restricted to the case that the group $G$ splits over a tamely ramified extension of the base field. This assumption is important for the construction of the group scheme $\mathcal{G}$ over $\mathcal{O}[u]$; it is always satisfied when $p \geq 5$ and $G$ is absolutely simple and is either adjoint or simply connected. A combination of our methods with the idea of splitting models from [48 can be used to also deal with groups that are Weil restrictions of scalars of tame groups down from a (possibly) wildly ramified extension. In particular, one can define local models and show Theorem 0.1 in some such cases 3 Of course, a general reductive group is isogenous to a product of Weil restrictions of absolutely simple adjoint groups and so this would allow us to handle most cases.

Let us now give an overview of the various sections of the paper and explain some aspects of our constructions. In $\S 1$ we discuss preliminaries on reductive groups over local fields; the main emphasis is on obtaining forms of a split group by descent as inner twists of a quasi-split form. In $\S 2$ we generalize this approach and give a certain reductive group scheme $\underline{G}$ over $\mathcal{O}\left[u, u^{-1}\right]$ which specializes to $G$ under the base change $\mathcal{O}\left[u, u^{-1}\right] \rightarrow F$ given by $u \mapsto \varpi$. Here, a crucial observation is that $\mathcal{O}\left[u, u^{-1}\right] \rightarrow F$ identifies the étale fundamental group of $\mathcal{O}\left[u, u^{-1}\right]$ with the tame quotient of the Galois group of $F$; our tameness hypothesis enters this way. Our construction of the "parahoric type" smooth affine group schemes $\mathcal{G}$ over $\mathcal{O}[u]$ is given in $\S 3$ (Theorem 3.1). Here is a brief outline of the construction of $\mathcal{G}$ : When $G$ is split over $F$ the existence of such a smooth group scheme $\mathcal{G}$ follows from [7]. However, showing that this is affine is not so straightforward. We do this in two stages. In the case that $K$ is contained in a hyperspecial subgroup, we realize $\mathcal{G}$ as a dilatation of the corresponding Chevalley group scheme over $\mathcal{O}[u]$ along a subgroup supported along $u=0$. In general, still for $G$ split, we reduce to the above case by using the fact that there is always a finite ramified extension $L / F$ such that the stabilizer of $x$ in $G(L)$ is contained in a hyperspecial subgroup. Next, we construct $\mathcal{G}$ when $G$ is quasi-split (and splits over a tamely ramified extension) by taking fixed points of a

\footnotetext{
${ }^{3}$ See the forthcoming thesis of B. Levin.
} 
corresponding group scheme for the split form. Finally, the general case is obtained by descent along an étale extension of $\mathcal{O}[u]$; this descent resembles a corresponding argument in [7]. In $\S 4$, we give several examples and eventually explain how, when $G$ is a classical group and $K$ is the connected stabilizer of a self-dual lattice chain (cf. [8], [10]), we can realize concretely the group schemes $\mathcal{G}$ as (the neutral components of) automorphisms of certain self-dual $\mathcal{O}[u]$-lattice chains.

In $\S 5$, we prove that the global affine Grassmannian $\operatorname{Gr}_{\mathcal{G}, X}$ is represented by an indscheme which is eventually shown to be ind-proper over $X$. The strategy here is to first demonstrate that we can find a faithful linear representation $\mathcal{G} \rightarrow \mathrm{GL}_{n}$ such that the quotient $\mathrm{GL}_{n} / \mathcal{G}$ is represented by a quasi-affine scheme. Given this we can reduce the proof of representability of $\mathrm{Gr}_{\mathcal{G}, X}$ to the standard case of $\mathcal{G}=\mathrm{GL}_{n}$. Here we are dealing with objects over the two-dimensional base $X=\mathbb{A}_{\mathcal{O}}^{1}$ and we need to work harder than in the usual situation in which the base is a smooth curve over a field. For example, it is not trivial to show that a smooth group scheme $\mathcal{G}$ with connected fibers over a two-dimensional regular base can be embedded as a closed subgroup scheme of $\mathrm{GL}_{n}$, as was proven by Thomason [70]. We can upgrade this to also show that there is such an embedding with $\mathrm{GL}_{n} / \mathcal{G}$ a quasi-affine scheme; this is done in the appendix $(\S 10)$. Also, general homogeneous spaces over a (regular) two dimensional base are not always represented by schemes; this complicates our proof. Raynaud asked whether the quotients of the form $\mathcal{H} / \mathcal{G}$, where $\mathcal{G}$ is a closed subgroup scheme of $\mathcal{H}$ and both $\mathcal{G}$ and $\mathcal{H}$ are smooth affine with connected fibers over a normal base, are represented by schemes. In the appendix, we also give an affirmative answer in the case when the base is excellent regular Noetherian and of Krull dimension two.

Our definition of the local models and certain generalizations is then given in $\S 6$. In $\S 7$, we describe the relation of local models to integral models of Shimura varieties and explain why in the PEL case our local models coincide with the (corrected) local models of Rapoport-Zink, Pappas-Rapoport and others. This uses our description of the group schemes $\mathcal{G}$ for classical groups given in $\S 4$ and the work of Rapoport and Zink. In $\S 8$ we show Theorem 0.1 and other related results. Finally, $\S 9$ is devoted to the study of nearby cycles and of the semi-simple trace of Frobenius; we show Theorem 0.3 , Theorem 0.4 and the identity (0.1).

Notations: In this paper, $\mathcal{O}$ denotes a discrete valuation ring with fraction field $F$ and perfect residue field $k$ of characteristic $p>0$. Most of the time $F$ will be a finite extension of the field of $p$-adic numbers. Usually, $G$ will denote a (connected) reductive group over $F$ and $\mathcal{B}(G, F)$ will be the Bruhat-Tits building of $G(F)$ (here by this we mean the "enlarged" building). As usual, we will denote by $G_{\text {der }}$, resp. $G_{\text {ad }}$, the derived subgroup, resp. the adjoint quotient of $G$, and by $G_{\mathrm{sc}}$ the simply-connected cover of $G_{\mathrm{der}}$. If $A$ is an affine algebraic group over a field $L$, we will use $\mathbb{X}^{\bullet}(A)$ (resp. $\mathbb{X}_{\bullet}(A)$ ) to denote the character group (resp. cocharacter group) over a separable closure $\bar{L}^{s}$. Then the Galois group $\operatorname{Gal}\left(\bar{L}^{s} / L\right)$ acts on $\mathbb{X}^{\bullet}(A)$ and $\mathbb{X}_{\bullet}(A)$. We will often write $X=\operatorname{Spec}(\mathcal{O}[u])$ for the affine line over $\operatorname{Spec}(\mathcal{O})$. We will denote by $R[[u]]$ the ring of formal power series ring in the variable $u$ with coefficients in $R, R((u))=R[[u]]\left[u^{-1}\right]$ is the corresponding ring of 
formal Laurent power series. If $Z$ is a set with an action of a group $\Gamma$, we will write $Z^{\Gamma}$ for the subset of elements $z$ of $Z$ which are fixed by the action: $\gamma \cdot z=z$ for all $\gamma \in \Gamma$.

Acknowledgements: The authors would like to warmly thank M. Rapoport, B. Conrad, T. Haines and B. Levin for useful discussions and comments.

\section{Preliminaries}

1.a. In all this chapter, unless we say to the contrary, we suppose that $F$ is either a $p$-adic field (i.e a finite extension of $\mathbb{Q}_{p}$ ), or the field of Laurent power series $k((t))$ with $k$ finite or algebraically closed of characteristic $p$. Recall $\mathcal{O}$ is the valuation ring of $F$. We fix a separable closure $\bar{F}^{s}$ of $F$ and denote by $\breve{F}$ the maximal unramified extension of $F$ in $\bar{F}^{s}$.

\section{1.b. Pinnings and quasi-split forms.}

1.b.1. We refer to 65] or [13] for background on reductive group schemes over a general base. Recall that a pinned Chevalley group over $\mathcal{O}$ is the data $\left(H, T_{H}, B_{H}, e\right)$ where $H$ is a Chevalley (reductive, connected, split) group scheme over $\mathcal{O}, B_{H}$ is a Borel subgroup scheme of $H, T_{H}$ a maximal split torus contained in $B_{H}$ and $e=\sum_{a \in \Delta} e_{a} \in \operatorname{Lie}\left(B_{H}\right)$, where $\Delta$ is the set of simple roots, $e_{a}$ is a generator of the rank $1 \mathcal{O}$-module $\operatorname{Lie}\left(U_{a}\right)$. Here, $U_{a}$ is the root group corresponding to $a$. The group of automorphisms $\Xi_{H}=\operatorname{Aut}\left(\Sigma_{H}\right)$ of the based root datum $\Sigma_{H}=\left(\mathbb{X}^{\bullet}\left(T_{H}\right), \Delta, \mathbb{X}_{\bullet}\left(T_{H}\right), \Delta^{\vee}\right)$ is canonically isomorphic to the group of automorphisms of $\left(H, T_{H}, B_{H}, e\right)$. We will call an element $\gamma$ of $\Xi_{H}$ a diagram automorphism of $\left(H, T_{H}, B_{H}, e\right)$.

1.b.2. Let $S$ be a (finite type, separated) scheme over $\operatorname{Spec}(\mathcal{O})$.

Definition 1.1. A pinned quasi-split (isotrivial) form of the group $H$ over $S$ is a quadruple $(\underline{G}, \underline{T}, \underline{B}, \underline{e})$, where $\underline{G}$ is a reductive group scheme over $S$ (see $[65]), \underline{T} \subset \underline{B}$ are closed subgroup schemes of $\underline{G}$ and $\underline{e} \in \operatorname{Lie}(\underline{B})$ a section such that locally for the finite étale topology of $S,(\underline{G}, \underline{T}, \underline{B}, \underline{e}) \simeq\left(H, T_{H}, B_{H}, e\right) \times_{\mathcal{O}} S$. We will denote the groupoid of pinned quasi-split (isotrivial) forms of the group $H$ over $S$ by $\mathrm{PQ}(H, S)$.

Remark 1.2. In this paper we only need isotrivial forms, i.e forms that split after a finite étale extension. We could also consider forms that split over a general étale extension but we choose not to do this here.

We will give a more combinatorial description of $\mathrm{PQ}(H, S)$. We say a scheme $\eta$ locally of finite presentation over $S$ is an isotrivial $\Xi_{H}$-torsor if it admits a right action of $\Xi_{H}$ and can be trivialized, i.e $\eta \times_{S} S^{\prime} \simeq \Xi_{H} \times S^{\prime}$, after a finite étale base change $S^{\prime} \rightarrow S$.

Proposition 1.3. The category $\mathrm{PQ}(H, S)$ is equivalent to the category given by quadruples $\left(T_{H}, B_{H}, e, \eta\right)$, where $\left(T_{H}, B_{H}, e\right)$ is a pinning of $H$ and $\eta \rightarrow S$ is an isotrivial $\Xi_{H}$-torsor. 
Proof. Indeed, given such a $\Xi_{H}$-torsor $\eta$ we can construct a reductive group scheme $\underline{G}$ over $S$ together with subgroup schemes $\underline{T}, \underline{B}$ and $\underline{e} \in \operatorname{Lie}(\underline{B})$ via twisting. Conversely, let $(\underline{G}, \underline{T}, \underline{B}, \underline{e})$ be a quadruple corresponding to an object of $\mathrm{PQ}(H, S)$. Let

$$
\eta\left(S^{\prime}\right)=\operatorname{Isom}\left(\left(\underline{G}_{S^{\prime}}, \underline{T}_{S^{\prime}}, \underline{B}_{S^{\prime}}, \underline{e}_{S^{\prime}}\right),\left(H, T_{H}, B_{H}, e\right) \times_{\mathcal{O}} S^{\prime}\right) .
$$

This is represented by a closed subscheme of $\operatorname{Isom}\left(\underline{G}, H_{S}\right)$; this isomorphism scheme is a $\underline{\text { Aut }}(H)$-torsor, and the latter group scheme is separated and smooth over $S$. Therefore, the morphism $\eta \rightarrow S$ is also separated and locally of finite presentation; the rest follows from the above.

1.b.3. Now let $s$ be a geometric point of $S$. Recall that there is an equivalence between the category of isotrivial $\Xi_{H}$-torsors on $S$ and the following groupoid: objects are continuous group homomorphisms $\rho: \pi_{1}(S, s) \rightarrow \Xi_{H}$, and the morphisms between $\rho_{1}$ and $\rho_{2}$ are elements $h \in \Xi_{H}$ such that $\rho_{2}=h \rho_{1} h^{-1}$. Therefore, we can describe $\operatorname{PQ}(H, S)$ as the category of quadruples $\left(T, B, e, \rho: \pi_{1}(S, s) \rightarrow \Xi_{H}\right)$.

This description of $\mathrm{PQ}(H, S)$ admits an immediate generalization as follows. Let $\Gamma$ be a profinite group. We let $\mathrm{PQ}(H, \Gamma)$ be the category of quadruples $\left(T, B, e, \rho: \Gamma \rightarrow \Xi_{H}\right)$. If $\pi: \pi_{1}(S, s) \rightarrow \Gamma$ is a continuous group homomorphism, there is a functor $\operatorname{PQ}(H, \Gamma) \rightarrow$ $\mathrm{PQ}(H, S)$ which is a full embedding if $\pi$ is surjective. We denote the image of this functor by $\mathrm{PQ}^{\Gamma}(H, S)$.

Now, suppose $S_{1} \rightarrow S_{2}$ is a morphism of schemes, and $s_{1}, s_{2}$ corresponding geometric points of $S_{1}$ and $S_{2}$ so that we have $\pi_{1}\left(S_{1}, s_{1}\right) \rightarrow \pi_{1}\left(S_{2}, s_{2}\right)$. Suppose that there is a surjective map $\pi_{1}\left(S_{2}, s_{2}\right) \rightarrow \Gamma$ such that the composition $\pi_{1}\left(S_{1}, s_{1}\right) \rightarrow \Gamma$ is also surjective. Then pullback along $S_{1} \rightarrow S_{2}$ induces an equivalence of categories

$$
\mathrm{PQ}^{\Gamma}\left(H, S_{2}\right) \stackrel{\sim}{\rightarrow} \mathrm{PQ}^{\Gamma}\left(H, S_{1}\right) .
$$

1.c. Fixed points. Here, we give some useful statements about the fixed points of an automorphism of a Chevalley group scheme.

1.c.1. If $\gamma$ is an automorphism of a separated scheme $Z \rightarrow S$, we will denote by $Z^{\gamma}$ the closed subscheme ([15, Prop. 3.1]) of fixed points for the action of $\gamma$ on $Z$ so that, by definition, we have $Z^{\gamma}(R)=Z(R)^{\gamma}:=\{z \in Z(R) \mid \gamma \cdot z=z\}$. We start with the useful:

Proposition 1.4. Let $H$ be a Chevalley group scheme (affine connected, reductive and split) over $\mathcal{O}$ with a pair $(T, B)$ of a maximal split torus $T$ and a Borel subgroup scheme $B$ that contains $T$. Suppose that $\gamma$ is an automorphism of $H$ of order e prime to $p$ that preserves both $T$ and $B$. Denote by $\Gamma=\langle\gamma\rangle$ the group generated by $\gamma$. Suppose that $E$ is a separably closed field which is an $\mathcal{O}$-algebra. Then the group of connected components $\pi_{0}\left(H_{E}^{\gamma}\right)$ of $H_{E}^{\gamma}$ is commutative with order that divides e and is independent of $E$.

Proof. We start with the case of tori. When $E=\mathbb{C}$ the isomorphisms in the Lemma below can be found in [35, Lemma 2.2]:

Lemma 1.5. Suppose that $T$ is a split torus over $\mathcal{O}$ which supports an action of the cyclic group $\Gamma=\langle\gamma\rangle$ of order e prime to $p$. Let $E$ be a separably closed field which is an 
O-algebra. We have

$$
\pi_{0}\left(\left(T_{E}\right)^{\gamma}\right) \simeq \mathrm{H}^{1}\left(\Gamma, \mathbb{X}_{\bullet}(T)\right), \quad \mathrm{H}^{1}(\Gamma, T(E)) \simeq \mathrm{H}^{2}\left(\Gamma, \mathbb{X}_{\bullet}(T)\right),
$$

and so in particular these groups are finite of order annihilated by e and are independent of $E$. The fppf sheaf over $\operatorname{Spec}(\mathcal{O})$ associated to $R \mapsto \mathrm{H}^{1}(\Gamma, T(R))$ is represented by a finite étale commutative group scheme of rank equal to the order of $\mathrm{H}^{2}\left(\Gamma, \mathbb{X}_{\bullet}(T)\right)$.

Proof. Consider the norm homomorphism $N=\prod_{i=0}^{e-1} \gamma^{i}: T \rightarrow T$. By comparing Lie algebras, we can see that the image $N\left(T_{E}\right)$ is the connected component $\left(T_{E}^{\gamma}\right)^{0}$ and so $\pi_{0}\left(T_{E}^{\gamma}\right)=T^{\gamma}(E) / N(T(E)) \simeq \mathrm{H}^{2}(\Gamma, T(E))=\mathrm{H}^{2}\left(\Gamma, \mathbb{X}_{\bullet}(T) \otimes_{\mathbb{Z}} E^{*}\right)$. Now consider the group $\mu_{e}(E)$ of roots of unity of order a power of $e$ in $E$. The quotient $E^{*} / \mu_{e}$ is uniquely divisible by powers of $e$ and we can conclude that $\mathrm{H}^{2}\left(\Gamma, \mathbb{X}_{\bullet}(T) \otimes_{\mathbb{Z}} E^{*}\right) \simeq$ $\mathrm{H}^{2}\left(\Gamma, \mathbb{X}_{\bullet}(T) \otimes_{\mathbb{Z}} \mu_{e} \infty\right)$. Since $\mu_{e}(E) \simeq \prod_{l \mid e} \mathbb{Q}_{l} / \mathbb{Z}_{l}$ and $\mathbb{Q}_{l}$ is similarly uniquely divisible, we obtain $\mathrm{H}^{2}\left(\Gamma, \mathbb{X}_{\bullet}(T) \otimes_{\mathbb{Z}} \mu_{e} \infty\right) \simeq \mathrm{H}^{1}\left(\Gamma, \mathbb{X}_{\bullet}(T) \otimes_{\mathbb{Z}} \prod_{l \mid e} \mathbb{Z}_{l}\right)=\mathrm{H}^{1}\left(\Gamma, \mathbb{X}_{\bullet}(T)\right)$. The proof of the second isomorphism is similar. Now, we can see that the fppf sheaf associated to the presheaf $R \mapsto \mathrm{H}^{1}(\Gamma, T(R))$ is given by the quotient group scheme $\operatorname{ker}(N) / T^{\gamma-1}$ and so the last statement also follows from the above.

Remark 1.6. Following [12], we call a split torus $T$ over $\mathcal{O}$ with $\Gamma$-action $\Gamma$-quasi-trivial, if $\mathbb{X}_{\bullet}(T)$ is a permutation $\Gamma$-module, i.e if $\mathbb{X} \bullet(T)$ has a $\mathbb{Z}$-basis which is stable under the action of $\Gamma$. We will call the split torus $T$ over $\mathcal{O}$ with $\Gamma$-action $\Gamma$-flasque if for all subgroups $\Gamma^{\prime} \subset \Gamma$, we have $\mathrm{H}^{1}\left(\Gamma^{\prime}, \mathbb{X}_{\bullet}(T)\right)=(0)$. Notice that by Shapiro's Lemma, if $T$ is $\Gamma$-quasi-trivial, then $T$ is also $\Gamma$-flasque. By the above lemma, if $T$ is $\Gamma$-flasque, then $T^{\gamma}$ is connected.

We now continue with the proof of Proposition 1.4 .

We will first discuss the cohomology set $\mathrm{H}^{1}(\Gamma, H(E))$. We will show that $\mathrm{H}^{1}(\Gamma, H(E))$ is finite of cardinality independent of $E$. For simplicity, we will omit $E$ from the notation and write $H$ instead of $H(E)$ etc. By [69, Lemma 7.3], every element of $H$ is $\gamma$-conjugate to an element of $B$. This implies that the natural map $\mathrm{H}^{1}(\Gamma, B) \rightarrow \mathrm{H}^{1}(\Gamma, H)$ is surjective. Now notice that $\mathrm{H}^{1}(\Gamma, T)=\mathrm{H}^{1}(\Gamma, B)$. (Indeed, $\mathrm{H}^{1}(\Gamma, U)=(0)$ because $\Gamma$ has order prime to the characteristic of $E$. In fact, for the same reason, $\mathrm{H}^{1}\left(\Gamma,{ }_{c} U\right)=(0)$ where ${ }_{c} U$ is $U$ with $\gamma$-action twisted by a cocycle $c$ in $Z^{1}(\Gamma, B)$. Since $T=B / U$, the long exact sequence of cohomology and [63, Cor. 2, I. §5] implies that $\mathrm{H}^{1}(\Gamma, B) \rightarrow \mathrm{H}^{1}(\Gamma, T)$ is injective. The splitting $T \rightarrow B$ shows that this map is also surjective.) Now suppose that $t, t^{\prime} \in T$ give cohomologous 1-cochains in $H$, i.e

$$
t^{\prime}=x t \gamma(x)^{-1}, \text { for some } x \in H .
$$

Using $H=\sqcup_{n \in N} U n U$, where $N$ is the normalizer of $T$, we write $x=u_{1} n u_{2}$. Then we get

$$
t^{\prime}=u_{1} n u_{2} t \gamma\left(u_{2}\right)^{-1} \gamma(n)^{-1} \gamma\left(u_{1}\right)^{-1}, \quad \text { or } \quad t^{\prime} \gamma\left(u_{1}\right) \gamma(n) \gamma\left(u_{2}\right)=u_{1} n u_{2} t .
$$

Since $T$ normalizes $U$, this implies $t^{\prime} \gamma(n)=n t$, so $t^{\prime}=n t \gamma(n)^{-1}$. This shows that two classes $\left[t^{\prime}\right],[t]$ in $\mathrm{H}^{1}(\Gamma, T)$ are identified in $\mathrm{H}^{1}(\Gamma, H)$ if and only if they are identified in $\mathrm{H}^{1}(\Gamma, N)$. Now use the exact sequence

$$
1 \rightarrow T^{\gamma} \rightarrow N^{\gamma} \rightarrow W^{\gamma} \rightarrow \mathrm{H}^{1}(\Gamma, T) \rightarrow \mathrm{H}^{1}(\Gamma, N),
$$


63. Prop. 39 (ii), I. $\S 5]$, and the above to conclude that $\mathrm{H}^{1}(\Gamma, H)$ can be identified with the set of orbits of a right action of $W^{\gamma}$ on $\mathrm{H}^{1}(\Gamma, T)$. This action is given as follows ([63, I. $\S 5,5.5])$. Suppose $w$ is in $W^{\gamma}$; lift $w$ to $n \in N$ and consider $t_{w}:=n^{-1} \gamma(n) \in T$. Then we set $[t] \cdot w=\left[n^{-1} t n \cdot t_{w}\right]=\left[n^{-1} t \gamma(n)\right]$. By Lemma 1.5, $\mathrm{H}^{1}(\Gamma, T) \simeq \mathrm{H}^{2}\left(\Gamma, X_{*}(T)\right)$ is independent of $E$. We can now easily see, by picking the lift $n$ of $w \in W^{\gamma}$ in $N(\mathcal{O})$, that the set of orbits $\mathrm{H}^{1}(\Gamma, T) / W^{\gamma}$ is also independent of the field $E$.

Let us now consider the group of connected components $\pi_{0}\left(H_{E}^{\gamma}\right)$.

Write $T_{\mathrm{der}}, T_{\mathrm{sc}}$, resp. $B_{\mathrm{der}}, B_{\mathrm{sc}}$, for the preimages of $T, B$, in the derived group $H_{\mathrm{der}}$, simply-connected cover $H_{\mathrm{sc}}$ of the derived group of $H$. The automorphism $\gamma$ gives corresponding automorphisms of $H_{\mathrm{der}}, H_{\mathrm{sc}}$ that preserve the pairs $\left(T_{\mathrm{der}}, B_{\mathrm{der}}\right),\left(T_{\mathrm{sc}}, B_{\mathrm{sc}}\right)$. By [69, Theorem 8.2], $H_{\mathrm{sc}}^{\gamma}$ is connected. Following the arguments in the proof of [12, Prop.-Def. 3.1] mutatis-mutandis, we see that we can find a central extension of reductive split groups with $\Gamma$-action over $\mathcal{O}$

$$
1 \rightarrow S \rightarrow H^{\prime} \rightarrow H \rightarrow 1
$$

where $S$ is a $\Gamma$-flasque torus, $H_{\text {der }}^{\prime}$ is simply connected and $H^{\prime} / H_{\text {der }}^{\prime}=D$ is a $\Gamma$-quasitrivial torus. In fact, we can make sure that $\gamma$ preserves a corresponding pair $\left(T^{\prime}, B^{\prime}\right)$ of $H^{\prime}$ with $1 \rightarrow S \rightarrow T^{\prime} \rightarrow T \rightarrow 1$. As before, we will now use the same letters to denote the base changes of these groups to $E$. We see that the exact sequence

$$
1 \rightarrow H_{\mathrm{der}}^{\prime} \rightarrow H^{\prime} \rightarrow D \rightarrow 1
$$

gives

$$
1 \rightarrow H_{\text {der }}^{\prime \gamma} \rightarrow H^{\prime \gamma} \rightarrow D^{\gamma} \rightarrow \mathrm{H}^{1}\left(\Gamma, H_{\text {der }}^{\prime}\right)
$$

By [69, Theorem 8.2], $H_{\text {der }}^{\prime \gamma}$ is connected; similarly $D^{\gamma}$ is connected by Lemma 1.5, cf. Remark 1.6. We can conclude that $H^{\prime \gamma}$ is connected. Now (1.2) gives

$$
1 \rightarrow S^{\gamma} \rightarrow H^{\prime \gamma} \rightarrow H^{\gamma} \rightarrow \mathrm{H}^{1}(\Gamma, S) \rightarrow \mathrm{H}^{1}\left(\Gamma, H^{\prime}\right) .
$$

Since both $S^{\gamma}$ and $H^{\prime \gamma}$ are connected (by Remark 1.6 and the above respectively), we obtain an exact sequence

$$
1 \rightarrow \pi_{0}\left(H^{\gamma}\right) \rightarrow \mathrm{H}^{1}(\Gamma, S) \rightarrow \mathrm{H}^{1}\left(\Gamma, H^{\prime}\right) .
$$

Since $S$ is central in $H^{\prime}$, the connecting map $\pi_{0}\left(H^{\gamma}\right) \rightarrow \mathrm{H}^{1}(\Gamma, S)$ is a group homomorphism and $\pi_{0}\left(H^{\gamma}\right)$ is identified with the subgroup of elements of $\mathrm{H}^{1}(\Gamma, S)$ that map to the trivial class in $\mathrm{H}^{1}\left(\Gamma, H^{\prime}\right)$. We can now conclude using the above results on $\mathrm{H}^{1}$ applied to $H=S, H=H^{\prime}$.

1.d. Reductive groups over local fields. In this section, we give some preliminaries on reductive groups over the local field $F$. In particular, we explain how we can present, using descent, such a group $G$ as a form of a split group $H$.

1.d.1. Let $G$ be a connective reductive group over $F$. Suppose that $G$ splits over the finite Galois extension $\tilde{F} / F$ with Galois group $\Gamma=\operatorname{Gal}(\tilde{F} / F)$. Denote by $H$ the Chevalley group scheme over $\mathbb{Z}$ which is the split form of $G$, i.e we have $G \otimes_{F} \tilde{F} \simeq H \otimes_{\mathbb{Z}} \tilde{F}$. In what follows, we fix a pinning $\left(T_{H}, B_{H}, e\right)$ of $H$ over $\mathcal{O}$. 
1.d.2. Let $A$ be a maximal $F$-split torus of $G$. Also, let $S$ be a maximal $\breve{F}$-split torus in $G$ which contains $A$ and is defined over $F$. Such a torus exists by [7, 5.1.12]. Let $T=Z_{G}(S), M=Z_{G}(A)$. Since $G_{\breve{F}}$ is quasi-split, $T$ is a maximal torus of $G$ which is defined over $F$ and splits over $\tilde{F}$.

As in [68, 16.4.7], by adjusting the isomorphism $G \otimes_{F} \tilde{F} \simeq H \otimes_{\mathbb{Z}} \tilde{F}$, we may identify $T$ with the maximal split torus $T_{H} \otimes_{\mathcal{O}} \tilde{F}$ of the split form $H$ of $G$ given by the pinning. We now represent the indexed root datum ([68, 16.2]) of $G$ over $F$ as a collection $\left(\mathbb{X}^{\bullet}(T), \Delta, \mathbb{X}_{\bullet}(T), \Delta^{\vee}, \Delta_{0}, \tau\right)$ where $\tau: \Gamma \rightarrow \Xi_{H}$ is a group homomorphism and $\Delta_{0}$ is a subset of $\Delta$ which is stable under the action of the group $\Gamma$ via $\tau$. Then using Proposition 1.3 we see that $\tau$ together with the pinning $\left(T_{H}, B_{H}, e\right)$ of $H$ gives a pinned quasi-split form $\left(G^{*}, T^{*}, B^{*}, e^{*}\right)$ of $H$ over $F$. Concretely,

$$
G^{*}=\left(\operatorname{Res}_{\tilde{F} / F}\left(H \otimes_{F} \tilde{F}\right)\right)^{\Gamma},
$$

where $\gamma \in \Gamma$ acts on $H \otimes_{F} \tilde{F}$ as $\tau(\gamma) \otimes \gamma$. This is the quasi-split form of $G$ over $F$.

Let $B_{H} \subset P_{0}$ be the standard parabolic subgroup of $H$ that corresponds to $\Delta_{0}$ and let $M_{0}$ be the Levi subgroup of $P_{0}$. Since $\tau$ leaves $\Delta_{0}$ stable, we can also similarly consider $M^{*}=M_{0}^{*}=\left(\operatorname{Res}_{\tilde{F} / F}\left(M_{0} \otimes_{\mathcal{O}} \tilde{F}\right)\right)^{\Gamma}$ which is also quasi-split over $F$. Then $T^{*}=\left(\operatorname{Res}_{\tilde{F} / F}\left(T_{H} \otimes_{\mathcal{O}} \tilde{F}\right)\right)^{\Gamma} \subset M^{*}$. Denote by $A^{*}$ the maximal $F$-split subtorus of $T^{*}$ and by $S^{*}$ the maximal $\breve{F}$-split subtorus of $T^{*}$ which is clearly defined over $F$ and which is a maximal $\breve{F}$-split torus of $G^{*}$.

By [68, 16.4.8], $G$ is an inner twist of $G^{*}$ which is given by a class $\mathrm{H}^{1}\left(\Gamma, G_{\mathrm{ad}}^{*}\left(\bar{F}^{s}\right)\right)$. Then there is a $\operatorname{Gal}\left(\bar{F}^{s} / F\right)$-stable $G_{\text {ad }}^{*}\left(\bar{F}^{s}\right)$-conjugacy class of isomorphisms $\psi: G_{\bar{F}^{s}} \stackrel{\sim}{\rightarrow} G_{\bar{F}^{s}}^{*}$ such that

$$
\gamma \mapsto \operatorname{Int}\left(g_{\gamma}\right)=\psi \cdot \gamma(\psi)^{-1}=\psi \gamma \psi^{-1} \gamma^{-1} \in G_{\mathrm{ad}}^{*}\left(\bar{F}^{s}\right)
$$

gives a 1-cocycle that represents the corresponding class in $\mathrm{H}^{1}\left(\Gamma, G_{\text {ad }}^{*}\left(\bar{F}^{s}\right)\right)$.

Notice that $G_{\breve{F}} \simeq G_{\breve{F}}^{*}$ since they are both quasi-split and they are inner forms of each other. This implies that we can realize the inner twist $G$ over $\breve{F}$. (In fact, over a finite extension of $F$ contained in $\breve{F}$.) More precisely, any inner twist $G_{\breve{F}} \otimes_{\breve{F}} \bar{F}^{s} \stackrel{\sim}{\rightarrow} G_{\breve{F}}^{*} \otimes_{\breve{F}} \bar{F}^{s}$ is conjugate over $\bar{F}^{s}$ to an isomorphism $\psi: G_{\breve{F}} \stackrel{\sim}{\rightarrow} G_{\breve{F}}^{*}$ (cf. [27, 11.2]). Then

$$
\operatorname{Int}(g)=\psi \cdot \sigma(\psi)^{-1}=\psi \sigma \psi^{-1} \sigma^{-1} \in G_{\text {ad }}^{*}(\breve{F})
$$

is an inner automorphism of $G_{\breve{F}}^{*}$, where $\sigma$ is the topological generator of $\operatorname{Gal}(\breve{F} / F) \simeq \hat{\mathbb{Z}}$ given by the lift of the (arithmetic) Frobenius. In what follows, we will choose this isomorphism $\psi$ more carefully. For this purpose, it is convenient to formulate the following:

Definition 1.7. A rigidification of $G$ is a triple $(A, S, P)$, where $A$ is a maximal $F$-split torus of $G, S \supset A$ a maximal $\breve{F}$-split torus of $G$ defined over $F$, and $P \supset M=Z_{G}(A)$ a minimal parabolic subgroup defined over $F$. A rigidified group over $F$ is a reductive group over $F$ together with a rigidification. The groupoid of rigidified groups over $F$ will be denoted by $\mathrm{RG}(F)$.

Lemma 1.8. The group $G_{\mathrm{ad}}(F)$ acts transitively on the set of rigidifications of $G$. 
Proof. Let $\left(A_{1}, S_{1}, P_{1}\right)$ and $\left(A_{2}, S_{2}, P_{2}\right)$ be two rigidifications. After conjugation, we can assume that $A_{1}=A_{2}, P_{1}=P_{2}$. Let $M=Z_{G}(A)$. Then we can replace $G$ by $M$. In addition, we can assume that it is of adjoint type; hence we reduce to the case that $G$ is adjoint anisotropic. We need to show that $S_{1}$ and $S_{2}$ are conjugate in this case. As $G$ is anisotropic, there is a unique parahoric group scheme of $G$ (the Iwahori group scheme, see [7, 5.2.7]); let us denote it by $\mathcal{P}$. By the construction of $\mathcal{P}$ in [7], we see that the Néron models $\mathcal{S}_{1}$ and $\mathcal{S}_{2}$ of $S_{1}$ and $S_{2}$ map naturally into $\mathcal{P}$ as closed subgroup schemes. Therefore, $\mathcal{S}_{1}(\breve{\mathcal{O}}), \mathcal{S}_{2}(\breve{\mathcal{O}}) \subset \mathcal{P}(\breve{\mathcal{O}})$. We can therefore choose $g$ in $\mathcal{P}(\breve{\mathcal{O}})$ such that $g S_{1} g^{-1}=S_{2}$. Since $\mathcal{P}$ is smooth and has connected fibers, $\mathrm{H}^{1}(\hat{\mathbb{Z}}, \mathcal{P}(\breve{\mathcal{O}}))=(1)($ see $[9,3.4$ Lemme 2]), and a standard argument shows that we can choose $g \in \mathcal{P}(\mathcal{O}) \subset G(F)$.

Proposition 1.9. Denote by $M^{\prime *}:=N_{G_{\mathrm{ad}}^{*}}\left(M^{*}\right) \cap P_{\mathrm{ad}}^{*}$ the Levi subgroup of $G_{\mathrm{ad}}^{*}$ that corresponds to $M^{*} \subset G^{*}$. The inner twist $G_{\bar{F}^{s}} \stackrel{\sim}{\rightarrow} G_{\bar{F}^{s}}^{*}$ is conjugate over $\bar{F}^{s}$ to an isomorphism $\psi: G_{\breve{F}} \stackrel{\sim}{\rightarrow} G_{\breve{F}}^{*}$ which is such that $\operatorname{Int}(g)=\psi \cdot \sigma(\psi)^{-1}$ lies in the normalizer $N_{M^{\prime *}}\left(S_{\mathrm{ad}}^{*}\right)$ of the torus $S_{\mathrm{ad}}^{*}$ in $M^{\prime *}$.

Proof. We pick up a rigidification $(A, S, P)$ of $G$. Recall $M=Z_{G}(A)$. Then $M^{*}$ is the unique standard Levi of $G^{*}$ (i.e. $T^{*} \subset M^{*}$ ) corresponding to $M$. The group $M^{*}$ is the quasi-split inner form of $M([68,16.4 .7])$. Indeed, let $P^{*}=B^{*} M^{*}=\left(\operatorname{Res}_{\tilde{F} / F}\left(P_{0} \otimes_{F} \tilde{F}\right)\right)^{\Gamma}$ (a standard parabolic subgroup in $G^{*}$ ) correspond to $P$. Observe that there is an inner twist $\psi: G_{\breve{F}} \stackrel{\sim}{\rightarrow} G_{\breve{F}}^{*}$ sending $P_{\breve{F}}$ to $P_{\breve{F}}^{*}$ and $M_{\breve{F}}$ to $M_{\breve{F}}^{*}$. Now $\operatorname{Int}(g)=\psi \sigma \psi^{-1} \sigma^{-1}$ preserves $M_{\breve{F}}^{*} \subset P_{\breve{F}}^{*}$. In particular, $g \in M^{\prime *}(\breve{F})$, where $M^{\prime *}:=N_{G_{\text {ad }}^{*}}\left(M^{*}\right) \cap P_{\text {ad }}^{*}$ is the corresponding Levi in $G_{\text {ad }}^{*}$. Therefore, $\operatorname{Int}(g): M_{\breve{F}}^{*} \rightarrow M_{\breve{F}}^{*}$ is inner. In particular, when restricted to the (connected) centers of $M$ and $M^{*}, \psi$ is an isomorphism of $F$ groups. Then it automatically sends $A=Z(M)_{s}^{0}$ (the maximal split torus in $Z(M)^{0}$ ) to $Z\left(M^{*}\right)_{s}^{0} \subset A^{*}$. By composing by an inner automorphism of $G^{*}(\breve{F})$ induced by an element in $M^{*}(\breve{F})$, we can further assume that $\psi: M_{\breve{F}} \rightarrow M_{\breve{F}}^{*}$ sends $S_{\breve{F}} \rightarrow S_{\breve{F}}^{*}$.

Let us denote

$$
N^{\prime *}=N_{M^{\prime *}}\left(T_{\mathrm{ad}}^{*}\right)=N_{M^{\prime *}}\left(S_{\mathrm{ad}}^{*}\right), \quad N_{\mathrm{ad}}^{*}:=\operatorname{Im}\left(N^{\prime *} \subset M^{\prime *} \rightarrow M_{\mathrm{ad}}^{*}\right) .
$$

Corollary 1.10. With the above notations, there is a unique class $\left[c^{\text {rig }}\right] \in \mathrm{H}^{1}\left(\hat{\mathbb{Z}}, N^{\prime *}(\breve{F})\right)$ whose image $[c]$ under

$$
\mathrm{H}^{1}\left(\hat{\mathbb{Z}}, N^{\prime *}(\breve{F})\right) \rightarrow \mathrm{H}^{1}\left(\hat{\mathbb{Z}}, M^{\prime *}(\breve{F})\right) \rightarrow \mathrm{H}^{1}\left(\hat{\mathbb{Z}}, G_{\mathrm{ad}}^{*}(\breve{F})\right) \subset \mathrm{H}^{1}\left(F, G_{\mathrm{ad}}^{*}\right),
$$

gives $G$ as an inner twist of its quasi-split form $G^{*}$, where we identify $\hat{\mathbb{Z}}=\operatorname{Gal}(\breve{F} / F)$ by sending 1 to the Frobenius $\sigma$.

Proof. The existence is given by Proposition 1.9 after choosing a rigidification $(G, A, S, P)$. Let $\left[c^{\prime}\right]$ be another class in $\mathrm{H}^{1}\left(\hat{\mathbb{Z}}, N^{\prime *}(\breve{F})\right)$ that also maps to $[c]$. Then via twisting $\left(G^{*}, S^{*}, M^{*}, P^{*}\right)$ by $c^{\prime}$, we obtain $\left(G^{\prime}, S^{\prime}, M^{\prime}, P^{\prime}\right)$, where $S^{\prime}$ is an $F$-torus of $G^{\prime}, M^{\prime} \supset T^{\prime}$ is a $F$-Levi subgroup of $G^{\prime}$ and $P^{\prime} \supset M^{\prime}$ is an $F$-parabolic subgroup of $G^{\prime}$. Let $A^{\prime}=Z\left(M^{\prime}\right)_{s}^{0}$ be an $F$-split torus of $G^{\prime}$. Since $G$ and $G^{\prime}$ are isomorphic as $F$-groups, $P^{\prime}$ is a minimal $F$-parabolic subgroup of $G^{\prime}, M^{\prime} \subset P^{\prime}$ is a minimal $F$-Levi subgroup of $G^{\prime}$, and therefore $A^{\prime}$ is a maximal $F$-split torus. In addition, $S^{\prime}$ is a maximal $\breve{F}$-split torus of $G^{\prime}$ defined 
over $F$. Therefore, there is an $F$-isomorphism $(G, A, S, P) \simeq\left(G^{\prime}, A^{\prime}, S^{\prime}, P^{\prime}\right)$ by Lemma 1.8. We can now conclude that $\left[c^{\prime}\right]$ coincides with $[c]$ given by Proposition [1.9,

Remark 1.11. Note that we do not claim that the map $\mathrm{H}^{1}\left(\hat{\mathbb{Z}}, N^{\prime *}(\breve{F})\right) \rightarrow \mathrm{H}^{1}\left(F, G_{\text {ad }}^{*}\right)$ is injective. In fact, the group $N^{\prime *}$ depends on $[c]$.

Remark 1.12. Let $(G, A, S, P)$ be a rigidified group over $F$; then $M=Z_{G}(A)$ is a minimal $F$-Levi of $G$ and $T=Z_{G}(S)$ a maximal torus of $G$. Let $M^{\prime}, T_{\text {ad }}$ be the images of $M, T$ in $G_{\text {ad }}$. We can see that the elements in $G_{\text {ad }}$ that fix the triple $(A, S, P)$ are those elements in $M^{\prime}$ that fix $T$ (or equivalently fix $T_{\mathrm{ad}}$ ). Therefore, we obtain the following exact sequence of $F$-groups

$$
1 \rightarrow N^{\prime} \rightarrow \operatorname{Aut}(G, A, S, P) \rightarrow \operatorname{Out}(G) \rightarrow 1
$$

where $N^{\prime}=N_{M^{\prime}}\left(T_{\mathrm{ad}}\right)$.

1.d.3. Now observe that the center of $M^{\prime *}=N_{G_{\text {ad }}^{*}}\left(M^{*}\right) \cap P_{\text {ad }}^{*}$ is connected. To see this, recall that $\mathbb{X}^{\bullet}\left(T_{\text {ad }}^{*}\right)=Q\left(G_{\text {ad }}^{*}\right)$, and $Q\left(M_{\text {ad }}^{*}\right)$ is just a direct factor of $Q\left(G_{\text {ad }}^{*}\right)$, where for a connected reductive group $L, Q(L)$ denote its absolute root lattice. Indeed, to specify $M_{\text {ad }}^{*}$ is the same as to choose a $\operatorname{Gal}(\bar{F} / F)$-stable subset $\Delta_{M^{*}} \subset \Delta_{G^{*}}$ of simple roots for $G_{\text {ad }}^{*}$, and $Q\left(M_{\text {ad }}^{*}\right)$ is the lattice generated by the simple roots in $\Delta_{M^{*}}$. Then the center of $M^{\prime *}$, which is the subgroup of $T_{\text {ad }}^{*}$ defined as the intersection of the kernel of all $a$ for $a \in \Delta_{M^{*}}$, is indeed an induced torus 4 , denoted by $Z^{*}$. We have

$$
1 \rightarrow Z^{*} \rightarrow N^{\prime *} \rightarrow N_{\text {ad }}^{*} \rightarrow 1
$$

Since $Z^{*}$ is induced, Hilbert theorem 90 and Shapiro's Lemma implies that $N^{\prime *}(\breve{F}) \rightarrow$ $N_{\text {ad }}^{*}(\breve{F})$ is surjective. Then, by [63, I. $\left.\S 5.7\right]$ we obtain an exact sequence of pointed sets

$$
\mathrm{H}^{1}\left(\hat{\mathbb{Z}}, N^{\prime *}(\breve{F})\right) \hookrightarrow \mathrm{H}^{1}\left(\hat{\mathbb{Z}}, N_{\text {ad }}^{*}(\breve{F})\right) \rightarrow \mathrm{H}^{2}\left(\hat{\mathbb{Z}}, Z^{*}(\breve{F})\right)
$$

with the first map injective. Write $Z^{*}=\prod_{j} \operatorname{Res}_{F_{j} / F} \mathbb{G}_{\mathrm{m}}$. Then using Shapiro's Lemma we see

$$
\mathrm{H}^{2}\left(\hat{\mathbb{Z}}, Z^{*}(\breve{F})\right)=\prod_{j} \operatorname{Br}\left(F_{j}\right) \simeq \prod_{j} \mathbb{Q} / \mathbb{Z}
$$

In the next subsection, we recall an explicit cocycle representing the image of $\left[c^{\text {rig }}\right]$ under $\mathrm{H}^{1}\left(\hat{\mathbb{Z}}, N^{\prime *}(\breve{F})\right) \rightarrow \mathrm{H}^{1}\left(\hat{\mathbb{Z}}, N_{\text {ad }}^{*}(\breve{F})\right)$.

1.d.4. Here we assume in addition that $F$ is a $p$-adic field, i.e a finite extension of $\mathbb{Q}_{p}$. Then we can choose the inner twist $\psi$ of Proposition 1.9 even more carefully: Indeed, recall that since $M=Z_{G}(A)$, the group $M_{\text {ad }}$ is anisotropic. Therefore, by [32, Satz 3], $M_{\text {ad }}$ is isomorphic to $\prod_{i} \operatorname{Res}_{E_{i} / F}\left(B_{i}^{\times} / E_{i}^{\times}\right)$, where $B_{i}$ are central division algebras of degree $m_{i}$ over $E_{i}$, and $E_{i}$ are finite extensions of $F$. Therefore, we have an isomorphism

$$
\rho: M_{\mathrm{ad}}^{*} \stackrel{\sim}{\rightarrow} \prod \operatorname{Res}_{E_{i} / F} \operatorname{PGL}\left(m_{i}\right),
$$

sending $S_{\text {ad }}^{*}$ to $\prod \operatorname{Res}_{E_{i} / F} \mathrm{D}_{m_{i}}$, where $\mathrm{D}_{n}$ is the standard torus in $\operatorname{PGL}(n, E)$.

\footnotetext{
${ }^{4}$ The torus $Z^{*}$ is induced because the Galois group permutes $\Delta_{G^{*}} \backslash \Delta_{M^{*}}$
} 
Lemma 1.13. Suppose that $B$ is a central division algebra over the local field $E$ of degree $n^{2}$ and with Brauer group invariant $r / n$ with $0<r<n$ and $\operatorname{gcd}(r, n)=1$. Denote by $\tau$ the permutation $(12 \cdots n)$. If $\varpi$ is a uniformizer of $E$ we let $\underline{n}:=\underline{n}_{r}(\varpi)$ be the element of $\operatorname{GL}(n, \breve{E})$ given by $\underline{n}\left(e_{i}\right)=e_{\tau^{r}(i)}$, if $i \neq n$, and $\underline{n}\left(e_{n}\right)=\varpi \cdot e_{\tau^{r}(n)}$. Then there is an isomorphism

$$
\psi: B \otimes_{E} \breve{E} \stackrel{\sim}{\rightarrow} M_{n \times n}(\breve{E})
$$

so that $\psi \sigma \psi^{-1} \sigma^{-1}=\operatorname{Int}(\underline{n})$.

Proof. Suppose that $E_{n} / E$ is an unramified extension of degree $n$ and $\sigma$ a generator of $\operatorname{Gal}\left(E_{n} / E\right)$. Then we can represent $B$ as the associative $E_{n}$-algebra with generator $\Pi$ and relations $\Pi^{n}=\varpi, a \cdot \Pi=\Pi \cdot \sigma(a)^{r}$, i.e

$$
B=\left\{\oplus_{i=0}^{n-1} E_{n} \cdot \Pi^{i} \mid \Pi^{n}=\varpi, a \cdot \Pi=\Pi \cdot \sigma(a)^{r}\right\} .
$$

Sending $\Pi$ to $\underline{n}=\underline{n}_{r}(\varpi)$ as above and $a \in E_{n}$ to the diagonal matrix $\left(a, \sigma(a), \ldots, \sigma^{n-1}(a)\right)$, gives an isomorphism $\psi: B \otimes_{E} E_{n} \stackrel{\sim}{\rightarrow} M_{n \times n}\left(E_{n}\right)$. The result now follows from an explicit calculation.

Suppose that the Brauer group invariant of $B_{i}$ is $r_{i} / m_{i}$ with $1 \leq r_{i}<m_{i}, \operatorname{gcd}\left(r_{i}, m_{i}\right)=$ 1. By the above lemma we can always choose an inner twist

$$
\psi^{\prime}:\left(M_{\mathrm{ad}}\right)_{\breve{F}} \stackrel{\sim}{\rightarrow}\left(\prod \operatorname{Res}_{E_{i} / F} \operatorname{PGL}\left(m_{i}\right)\right)_{\breve{F}},
$$

such that the element $g \in \prod \operatorname{PGL}\left(m_{i}, E_{i} \otimes_{F} \breve{F}\right)$ given by $\operatorname{Int}(g)=\psi^{\prime} \sigma \psi^{\prime-1} \sigma^{-1}$ satisfies $g=\prod g_{i}$, and $g_{i}=\underline{n}_{r_{i}}\left(\varpi_{i}\right)$ where $\varpi_{i}$ is a uniformizer of $E_{i}$. Combining with the discussion in 1.d.3, we obtain that

Proposition 1.14. Assume that $F$ is a p-adic field and choose an isomorphism (1.9). Then we can find $\psi: G_{\breve{F}} \stackrel{\sim}{\rightarrow} G_{\breve{F}}^{*}$ as in Proposition 1.9, such that in addition the image of $\operatorname{Int}(g)$ under $M^{\prime *}(\breve{F}) \rightarrow M_{\text {ad }}^{*}(\breve{F})$ is given by the element $g=\prod_{i} g_{i}$ with $g_{i}$ as above.

\section{Reductive groups over $\mathcal{O}\left[u, u^{-1}\right]$}

Recall $G$ is a connected reductive group over $F$. We will assume that:

(Tameness hypothesis) $G$ splits over a finite tamely ramified extension $\tilde{F} / F$.

Let $\varpi$ be a uniformizer of $\mathcal{O}$. Our goal in this section is to construct, when $F$ is a $p$-adic field, a reductive group scheme $\underline{G}$ over $\operatorname{Spec}\left(\mathcal{O}\left[u^{ \pm 1}\right]\right)$ which extends $G$ in the sense that its base change

$$
\underline{G} \otimes_{\mathcal{O}\left[u^{ \pm 1}\right]} F, \quad u \mapsto \varpi,
$$

is isomorphic to $G$. This is done by following the procedure of the construction of $G$ from its split form $H$ which was described in $\$ 1 . d$, we first extend the quasi-split form $G^{*}$ to $\underline{G}^{*}$ and then give $\underline{G}$ by an appropriate descent. (We write $\mathcal{O}\left[u^{ \pm 1}\right]$ instead of $\mathcal{O}\left[u, u^{-1}\right]$ for brevity.) 
2.a. The tame splitting field. We assume that $F$ is either a $p$-adic field, or $F=k((t))$ with $k$ finite and that in either case the residue field $k$ has cardinality $q=p^{m}$. Denote by $\tilde{F}_{0}$ the maximal unramified extension of $F$ that is contained in $\tilde{F}$ and by $\tilde{\mathcal{O}}_{0}$, $\tilde{\mathcal{O}}$ the valuation rings of $\tilde{F}_{0}, \tilde{F}$ respectively. Set $e=\left[\tilde{F}: \tilde{F}_{0}\right]$ (which is then prime to $p$ ) and let $\gamma_{0}$ be a generator of $\operatorname{Gal}\left(\tilde{F} / \tilde{F}_{0}\right)$. Recall that by Steinberg's theorem, the group $G_{\breve{F}}:=G \otimes_{F} \breve{F}$ is quasi-split. By possibly enlarging the splitting field $\tilde{F}$, we can now assume that

- $G_{\tilde{F}_{0}}$ is quasi-split,

- $\tilde{F} / F$ is Galois with group $\Gamma=\operatorname{Gal}(\tilde{F} / F)=\langle\sigma\rangle \rtimes\left\langle\gamma_{0}\right\rangle$ which is the semi-direct product of $\langle\sigma\rangle \simeq \mathbb{Z} /(r)$, where $\sigma$ is a lift of the (arithmetic) Frobenius Frob $_{q} \in$ $\operatorname{Gal}\left(\tilde{F}_{0} / F\right)$, with the normal inertia subgroup $I:=\operatorname{Gal}\left(\tilde{F} / \tilde{F}_{0}\right)=\left\langle\gamma_{0}\right\rangle \simeq \mathbb{Z} /(e)$, with relation $\sigma \gamma_{0} \sigma^{-1}=\gamma_{0}^{q}$,

- there is a uniformizer $\tilde{\varpi}$ of $\tilde{\mathcal{O}}$ such that $\tilde{\varpi}^{e}=\varpi$.

Without further mention, we will assume that the extension $\tilde{F} / F$ is as above. Then we also have $\tilde{\mathcal{O}}=\tilde{\mathcal{O}}_{0}[\tilde{\varpi}] \simeq \tilde{\mathcal{O}}_{0}[x] /\left(x^{e}-\varpi\right)$ and $\tilde{\mathcal{O}}_{0}$ contains a primitive $e$-th root of unity $\zeta=\gamma_{0}(\tilde{\varpi}) \tilde{\varpi}^{-1}$.

2.b. Covers of $\mathcal{O}[u]$ and $\mathcal{O}\left[u^{ \pm 1}\right]$. Suppose that $F$ is a $p$-adic field with ring of integers $\mathcal{O}$ and residue field $k=\mathbb{F}_{q}$. Let $\tilde{F} / F$ be a finite tamely ramified Galois extension of $F$ as in 2.a with Galois group $\Gamma=\left\langle\gamma_{0}, \sigma \mid \sigma^{r}=\gamma_{0}^{e}=1, \sigma \gamma_{0} \sigma^{-1}=\gamma_{0}^{q}\right\rangle$. The maximal tamely ramified extension $F^{t}$ of $F$ in $\bar{F}$ is the union of fields $\tilde{F}$ as above and its Galois group

$$
\Gamma^{t}:=\operatorname{Gal}\left(F^{t} / F\right) \simeq \prod_{l \neq p} \mathbb{Z}_{l}(1) \rtimes \hat{\mathbb{Z}}
$$

is the projective limit of the corresponding groups $\Gamma$.

2.b.1. Consider the affine line $\mathbb{A}_{\mathcal{O}}^{1}=\operatorname{Spec}(\mathcal{O}[u])$ and its cover

$$
\pi: \mathbb{A}_{\tilde{\mathcal{O}}_{0}}^{1}=\operatorname{Spec}\left(\tilde{\mathcal{O}}_{0}[v]\right) \rightarrow \mathbb{A}_{\mathcal{O}}^{1}=\operatorname{Spec}(\mathcal{O}[u])
$$

given by $u \mapsto v^{e}$. The (abstract) group $\Gamma$ described as above acts on $\tilde{\mathcal{O}}_{0}[v]$ by

$$
\sigma\left(\sum_{i} a_{i} v^{i}\right)=\sum_{i} \sigma\left(a_{i}\right) v^{i}, \quad \gamma_{0}\left(\sum_{i} a_{i} v^{i}\right)=\sum_{i} a_{i} \zeta^{i} v^{i}
$$

where $\zeta$ is the primitive $e$-th root of unity $\gamma_{0}(\tilde{\varpi}) \tilde{\varpi}^{-1}$ in $\tilde{\mathcal{O}}_{0}$. We have $\tilde{\mathcal{O}}_{0}[v]^{\Gamma}=\mathcal{O}[u]$ and $\pi$ is a $\Gamma$-cover ramified over $u=0$. The restriction of $\pi$ over the open subscheme $u \neq 0$ gives a $\Gamma$-torsor

$$
\pi_{0}: \operatorname{Spec}\left(\tilde{\mathcal{O}}_{0}\left[v^{ \pm 1}\right]\right) \rightarrow \operatorname{Spec}\left(\mathcal{O}\left[u^{ \pm 1}\right]\right)=\mathbb{G}_{m \mathcal{O}}
$$

Notice that base changing the $\Gamma$-cover $\pi$ via the map $\mathcal{O}[u] \rightarrow F$ given by $u \mapsto \varpi$ gives $\operatorname{Spec}(\tilde{F}) \rightarrow \operatorname{Spec}(F)$ with its Galois action. In this way, we realize $\Gamma^{t}$ as a quotient of the fundamental group of $\mathbb{G}_{m \mathcal{O}}$ such that the composed map $\operatorname{Gal}(\bar{F} / F) \rightarrow \Gamma^{t}$ coincides with the tame quotient of $\operatorname{Gal}(\bar{F} / F)$. In fact, we can easily see that $\pi_{1}\left(\mathbb{G}_{\mathrm{m} \mathcal{O}}, \operatorname{Spec}(\bar{F})\right) \rightarrow \Gamma^{t}$ is an isomorphism. 
2.b.2. Suppose that we take $F=\mathbb{Q}_{p}$ and that $L$ is a tamely ramified finite extension of $\mathbb{Q}_{p}$. Let $\tilde{F}$ be a Galois extension of $F=\mathbb{Q}_{p}$ that contains $L$ and satisfies the assumptions of 2. a with $\Gamma=\operatorname{Gal}\left(\tilde{F} / \mathbb{Q}_{p}\right)$. In particular, $\tilde{F}_{0}$ is the maximal unramified extension of $\mathbb{Q}_{p}$ contained in $\tilde{F}$. Denote by $L_{0}$ the maximal unramified extension of $\mathbb{Q}_{p}$ contained in $L$ and suppose $L \simeq L_{0}[x] /\left(x^{e_{L}}-p \cdot c\right), c \in \mathcal{O}_{L_{0}}^{*}$. Let $\Gamma_{L}$ the subgroup of $\Gamma$ that fixes $L$. We can then see that there is a $\mathbb{Z}_{p}[u]$-algebra homomorphism

$$
\left(\tilde{\mathcal{O}}_{0}[v]\right)^{\Gamma_{L}} \simeq \mathcal{O}_{L_{0}}[w]
$$

in which the right hand side is a $\mathbb{Z}_{p}[u]$-algebra via $u \mapsto w^{e_{L}} \cdot c^{-1}$.

2.c. Groups over $\mathcal{O}\left[u^{ \pm 1}\right]$. We will now give the construction of the group schemes $\underline{G}$ over $\mathcal{O}\left[u^{ \pm 1}\right]$.

2.c.1. Recall that $G$ is a connected reductive group over the $p$-adic field $F$ which splits over the tame extension $\tilde{F} / F$ as in $\$ 2$.a. We will use the notations of $\$ 1$. In particular, $H$ over $\mathcal{O}$ is the Chevalley (split) form of $G$; we pick a pinning on $H$ as in 1 .b.1. Then the indexed root datum for $G$ gives a group homomorphism $\tau: \Gamma=\operatorname{Gal}(\tilde{F} / F) \rightarrow \operatorname{Aut}_{\mathcal{O}}(H)$ and using 81. b.3 we obtain the pinned quasi-split group $\left(G^{*}, T^{*}, B^{*}, e\right)$ over $F$.

We apply the equivalence of categories (1.1) to the following case: Let $S_{2}=\mathbb{G}_{m \mathcal{O}}=$ $\operatorname{Spec}\left(\mathcal{O}\left[u^{ \pm 1}\right]\right)$ and $s=S_{1}=\operatorname{Spec}(F) \rightarrow S_{2}$ be the point given by $u=\varpi$. Let $\bar{s}=\operatorname{Spec}(\bar{F})$ be a geometric point over $s$. Then we obtain an equivalence

$$
\mathrm{PQ}\left(H, \mathbb{G}_{m \mathcal{O}}\right) \simeq \mathrm{PQ}^{\Gamma^{t}}\left(H, \mathbb{G}_{m \mathcal{O}}\right) \stackrel{\sim}{\rightarrow} \mathrm{PQ}^{\Gamma^{t}}(H, F) .
$$

We choose a quasi-inverse of this functor and therefore, for any pinned quasi-split group $\left(G^{*}, T^{*}, B^{*}, e^{*}\right)$ over $F$, we have $\left(\underline{G}^{*}, \underline{T}^{*}, \underline{B}^{*}, \underline{e}^{*}\right)$ over $\mathcal{O}\left[u^{ \pm 1}\right]$ together with an isomorphism $\left(\underline{G}^{*}, \underline{T}^{*}, \underline{B}^{*}, \underline{e}^{*}\right) \otimes_{\mathcal{O}\left[u, u^{-1}\right]} F \simeq\left(G^{*}, T^{*}, B^{*}, e^{*}\right)$.

In particular, $G^{*}$ is the reductive group scheme over $\mathcal{O}\left[u^{ \pm 1}\right]$ which is the twisted form of $\underline{H}=H \otimes_{\mathcal{O}} \mathcal{O}\left[u^{ \pm 1}\right]$ obtained from the $\Gamma$-torsor $\pi_{0}: \operatorname{Spec}\left(\tilde{\mathcal{O}}_{0}\left[v^{ \pm 1}\right]\right) \rightarrow \operatorname{Spec}\left(\mathcal{O}\left[u^{ \pm 1}\right]\right)$ using $\tau$. More concretely,

$$
\underline{G}^{*}=\left(\operatorname{Res}_{\tilde{\mathcal{O}}_{0}\left[v^{ \pm 1}\right] / \mathcal{O}\left[u^{ \pm 1}\right]}\left(H \otimes \mathcal{O} \tilde{\mathcal{O}}_{0}\left[v^{ \pm 1}\right]\right)\right)^{\Gamma}
$$

where $\gamma \in \Gamma$ acts diagonally via $\tau(\gamma) \otimes \gamma$. The same construction applies to other groups that inherit a pinning from $G^{*}$; for example, it applies to the Levi subgroup $M_{0}$ that corresponds to the $\Gamma$-stable subset $\Delta_{0} \subset \Delta$. We obtain

$$
\underline{M}^{*}=\left(\operatorname{Res}_{\tilde{\mathcal{O}}_{0}\left[v^{ \pm 1}\right] / \mathcal{O}\left[u^{ \pm 1}\right]}\left(M_{0} \otimes_{\mathcal{O}} \tilde{\mathcal{O}}_{0}\left[v^{ \pm 1}\right]\right)\right)^{\Gamma} .
$$

Again base changing along $u \mapsto \varpi$ gives a group canonically isomorphic to $M^{*} \subset G^{*}$.

2.c.2. The goal of the rest of this chapter is to explain how to construct a quadruple of group schemes $(\underline{G}, \underline{A}, \underline{S}, \underline{P})$, whose specialization along $u=\varpi$ gives rise to $G$ together with a rigidification $(A, S, P)$.

First as above, we obtain adjoint quasi-split forms $\underline{M}_{\mathrm{ad}}^{*}, \underline{G}_{\mathrm{ad}}^{*}$ over $\mathcal{O}\left[u^{ \pm 1}\right]$ that specialize to $M_{\mathrm{ad}}^{*}, G_{\mathrm{ad}}^{*}$ over $F$ after the base change $u \mapsto \varpi$. Then let $\underline{N^{\prime *}}=N_{\underline{M}^{\prime *}}\left(\underline{T}_{\mathrm{ad}}^{*}\right)$ and $\underline{N}_{\mathrm{ad}}^{*}=\operatorname{Im}\left(\underline{N}^{\prime *} \rightarrow \underline{M}_{\mathrm{ad}}^{*}\right)$. We also obtain a short exact (central) sequence

$$
1 \rightarrow \underline{Z}^{*} \rightarrow \underline{N}^{*} \rightarrow \underline{N}_{\mathrm{ad}}^{*} \rightarrow 1
$$


which specializes to (1.6). We will consider the base change of these groups after the étale extension $\mathcal{O}\left[u^{ \pm 1}\right] \rightarrow \breve{\mathcal{O}}\left[u^{ \pm 1}\right]$. For simplicity, we will sometimes omit this base change from the notation. Here $\underline{Z}^{*}$ is an induced torus and by (2.2) we have

$$
\underline{Z}^{*} \otimes_{\mathcal{O}} \breve{\mathcal{O}} \simeq \prod_{j} \operatorname{Res}_{\breve{\mathcal{O}}\left[w^{ \pm 1}\right] / \breve{\mathcal{O}}\left[u^{ \pm 1}\right]} \mathbb{G}_{\mathrm{m}}
$$

where $w^{d_{j}}=u c_{j}^{-1}$ for $p \nmid d_{j}$. By applying $\operatorname{Pic}\left(\breve{\mathcal{O}}\left[w^{ \pm 1}\right]\right)=(1)$ and Hilbert's theorem 90 we see that $\mathrm{H}_{\text {et }}^{1}\left(\operatorname{Spec}\left(\breve{\mathcal{O}}\left[u^{ \pm 1}\right]\right), \underline{Z}^{*}\right)=(1)$. This gives that

$$
\underline{N^{\prime *}}\left(\breve{\mathcal{O}}\left[u^{ \pm 1}\right]\right) \rightarrow \underline{N}_{\text {ad }}^{*}\left(\breve{\mathcal{O}}\left[u^{ \pm 1}\right]\right)
$$

is surjective. The exact cohomology sequence for a central quotient now gives that

$$
\mathrm{H}^{1}\left(\hat{\mathbb{Z}}, \underline{N^{\prime *}}\left(\breve{\mathcal{O}}\left[u^{ \pm 1}\right]\right)\right) \hookrightarrow \mathrm{H}^{1}\left(\hat{\mathbb{Z}}, \underline{N}_{\text {ad }}^{*}\left(\breve{\mathcal{O}}\left[u^{ \pm 1}\right]\right)\right) \rightarrow \mathrm{H}^{2}\left(\hat{\mathbb{Z}}, \underline{Z}^{*}\left(\breve{\mathcal{O}}\left[u^{ \pm 1}\right]\right)\right)
$$

is an exact sequence of pointed sets. There are natural maps between the exact sequences (2.8) and (1.7) obtained by sending $\breve{\mathcal{O}}\left[u^{ \pm 1}\right] \rightarrow \breve{F}$ via $u \mapsto \varpi$.

Recall the description of $M_{\mathrm{ad}}$ and the isomorphism (1.9) that follow from Kneser's theorem. Using (2.3) we obtain that there is an isomorphism

$$
\underline{\rho}: \underline{M}_{\mathrm{ad}}^{*} \stackrel{\sim}{\longrightarrow} \prod_{i} \operatorname{Res}_{\tilde{\mathcal{O}}_{0}\left[v^{ \pm 1}\right]^{\Gamma_{i}} / \mathcal{O}\left[u^{ \pm 1}\right]} \operatorname{PGL}\left(m_{i}\right)
$$

where $\Gamma_{i} \subset \Gamma$ are subgroups of finite index in the Galois group $\Gamma$ of $\tilde{\mathcal{O}}_{0}\left[v^{ \pm 1}\right] / \mathcal{O}\left[u^{ \pm 1}\right]$ which specializes under $u \mapsto \varpi$, to the isomorphism (1.9).

2.c.3. Now recall that the anisotropic kernel $M$ (an inner form of $M^{*}$ ) gives a welldefined class $[c(M)]$ in $\mathrm{H}^{1}\left(\hat{\mathbb{Z}}, M_{\text {ad }}^{*}(\breve{F})\right)$. Applying Proposition 1.10 to $M$ and the exact sequence (1.7), we see that $[c(M)]$ has the following properties:

1) It is the image of a unique class $\left[c^{\text {rig }}\right]$ in $\mathrm{H}^{1}\left(\hat{\mathbb{Z}}, N^{\prime *}(\breve{F})\right)$, and,

2) The image of $\left[c^{\text {rig }}\right]$ under $\mathrm{H}^{1}\left(\hat{\mathbb{Z}}, N^{\prime *}(\breve{F})\right) \rightarrow \mathrm{H}^{1}\left(\hat{\mathbb{Z}}, G_{\text {ad }}^{*}(\breve{F})\right)$ gives the inner twist $G$ of $G^{*}$.

We have $\mathrm{H}^{2}\left(\hat{\mathbb{Z}}, \breve{\mathcal{O}}^{*}\right)=(1)$, hence $\mathrm{H}^{2}\left(\hat{\mathbb{Z}}, \breve{\mathcal{O}}\left[u^{ \pm 1}\right]^{*}\right)=\mathrm{H}^{2}\left(\hat{\mathbb{Z}}, \breve{\mathcal{O}}^{*} \times u^{\mathbb{Z}}\right) \simeq \mathrm{H}^{2}(\hat{\mathbb{Z}}, \mathbb{Z}) \simeq \mathbb{Q} / \mathbb{Z}$, and similarly $\operatorname{Br}(F)=\mathrm{H}^{2}\left(\hat{\mathbb{Z}}, \breve{F}^{*}\right)=\mathrm{H}^{2}\left(\hat{\mathbb{Z}}, \breve{\mathcal{O}}^{*} \times \varpi^{\mathbb{Z}}\right) \simeq \mathbb{Q} / \mathbb{Z}$. Therefore, $u \mapsto \varpi$ gives an isomorphism $\mathrm{H}^{2}\left(\hat{\mathbb{Z}}, \breve{\mathcal{O}}\left[u^{ \pm 1}\right]^{*}\right) \stackrel{\sim}{\rightarrow} \mathrm{H}^{2}\left(\hat{\mathbb{Z}}, \breve{F}^{*}\right)$. Similarly, using (2.6) $)$, and Shapiro's Lemma we obtain that $u \mapsto \varpi$ gives an isomorphism

$$
\mathrm{H}^{2}\left(\hat{\mathbb{Z}}, \underline{Z}^{*}\left(\breve{\mathcal{O}}\left[u^{ \pm 1}\right]\right)\right) \stackrel{\sim}{\rightarrow} \mathrm{H}^{2}\left(\hat{\mathbb{Z}}, Z^{*}(\breve{F})\right) .
$$

Note that using (2.9), (2.2) and Shapiro's lemma we obtain an isomorphism

$$
\mathrm{H}^{1}\left(\hat{\mathbb{Z}}, \underline{M}_{\mathrm{ad}}^{*}\left(\breve{\mathcal{O}}\left[u^{ \pm 1}\right]\right) \stackrel{\sim}{\rightarrow} \prod_{i} \mathrm{H}^{1}\left(\hat{\mathbb{Z}}, \mathrm{PGL}_{m_{i}}\left(\breve{\mathcal{O}}\left[w_{i}^{ \pm 1}\right]\right)\right)\right. \text {. }
$$

The constructions explained in 4.c.1 and 4.c.3 produce Azumaya algebras $\mathcal{B}_{i}$ over $\mathcal{O}\left[w_{i}^{ \pm 1}\right]$ that under $w \mapsto \varpi_{i}$ specialize to the central division algebras $B_{i}$ that appear in the decomposition of the anisotropic kernel $M_{\text {ad }}$. These Azumaya algebras split over $\breve{\mathcal{O}}\left[w_{i}^{ \pm 1}\right]$; hence, by using the isomorphism (2.11), we obtain a well-defined class $[\underline{c}] \in \mathrm{H}^{1}\left(\hat{\mathbb{Z}}, \underline{M}_{\text {ad }}^{*}\left(\breve{\mathcal{O}}\left[u^{ \pm 1}\right]\right)\right)$ that specializes to $[c(M)] \in \mathrm{H}^{1}\left(\hat{\mathbb{Z}}, M_{\text {ad }}^{*}(\breve{F})\right)$. 
Now, using Lemma 1.13, the explicit constructions of 4.c.1, 4.c.3 and our discussion in $\$ 2 . \mathrm{C}$, the class $[\underline{c}]$ corresponding to the product of Azumaya algebras in fact comes from a canonical class $\left[\underline{c}^{\text {rig }}\right]$ in $\mathrm{H}^{1}\left(\hat{\mathbb{Z}}, \underline{N}_{\text {ad }}^{*}\left(\breve{\mathcal{O}}\left[u^{ \pm 1}\right]\right)\right)$, whose specialization to $\mathrm{H}^{1}\left(\hat{\mathbb{Z}}, N_{\text {ad }}^{*}(\breve{F})\right)$ is the image of $\left[c^{\text {rig }}\right]$ in Corollary 1.10 under the map $\mathrm{H}^{1}\left(\hat{\mathbb{Z}}, N^{\prime *}(\breve{F})\right) \rightarrow \mathrm{H}^{1}\left(\hat{\mathbb{Z}}, N_{\text {ad }}^{*}(\breve{F})\right)$.

By (1.7), $[c]$ maps to the trivial class in $\mathrm{H}^{2}\left(\hat{\mathbb{Z}}, Z^{*}(\breve{F})\right)$. Using (2.8) and the isomorphism (2.10) we see that $\left[\underline{c}^{\text {rig }}\right]$ belongs to $\mathrm{H}^{1}\left(\hat{\mathbb{Z}}, \underline{N}^{*}\left(\breve{\mathcal{O}}\left[u^{ \pm 1}\right]\right)\right)$ and maps to the class $\left[c^{\text {rig }}\right]$ in $\mathrm{H}^{1}\left(\hat{\mathbb{Z}}, N^{\prime *}(\breve{F})\right)$ under the specialization $u \mapsto \varpi$.

Remark 2.1. In fact, one can show that specialization $u \mapsto \varpi$ induces isomorphisms

$$
\mathrm{H}^{1}\left(\hat{\mathbb{Z}}, \underline{N^{\prime *}}\left(\breve{\mathcal{O}}\left[u^{ \pm 1}\right]\right)\right) \stackrel{\sim}{\rightarrow} \mathrm{H}^{1}\left(\hat{\mathbb{Z}}, N^{\prime *}(\breve{F})\right), \quad \mathrm{H}^{1}\left(\hat{\mathbb{Z}}, \underline{N}_{\text {ad }}^{*}\left(\breve{\mathcal{O}}\left[u^{ \pm 1}\right]\right)\right) \stackrel{\sim}{\rightarrow} \mathrm{H}^{1}\left(\hat{\mathbb{Z}}, N_{\text {ad }}^{*}(\breve{F})\right) .
$$

We do not use this fact here.

2.c.4. The class $\left[\underline{c}^{\text {rig }}\right]$ allows us to define an inner twist $\underline{G}$ of $\underline{G}^{*}$ : Let $\underline{c}^{\text {rig }}$ be a 1-cocycle representing this class and denote by $\operatorname{Int}(\mathbf{g})$ the element in $\underline{M^{\prime *}}\left(\breve{\mathcal{O}}\left[u^{ \pm 1}\right]\right) \subset \underline{G}_{\text {ad }}^{*}\left(\breve{\mathcal{O}}\left[u^{ \pm 1}\right]\right)$ which is the value at 1 of this cocycle. We define

$$
\underline{G}(R)=\underline{G}^{*}\left(\breve{\mathcal{O}}\left[u^{ \pm 1}\right] \otimes_{\mathcal{O}\left[u^{ \pm 1}\right]} R\right)^{\hat{\mathbb{Z}}}
$$

where the action of the topological generator 1 of $\hat{\mathbb{Z}}$ is given by $\operatorname{Int}(\mathbf{g}) \cdot \sigma$. (In other words, $\underline{G}$ is given by the Weil descent datum obtained from the automorphism $\operatorname{Int}(\mathbf{g}) \cdot \sigma$.) By descent, $\underline{G}$ is a reductive group over $\mathcal{O}\left[u^{ \pm 1}\right]$ with base change to $\breve{\mathcal{O}}\left[u^{ \pm 1}\right]$ isomorphic to $\underline{G}^{*} \otimes_{\mathcal{O}} \mathcal{O}$

In fact, we obtain more. Namely, we have

$$
(\underline{G}, \underline{A}, \underline{S}, \underline{T}, \underline{M}, \underline{P}),
$$

where $(\underline{G}, \underline{T}, \underline{M}, \underline{P})$ is obtained from $\left(\underline{G}^{*}, \underline{T}^{*}, \underline{M}^{*}, \underline{P}^{*}\right)$ via twisting by the cocycle $\underline{c}^{\text {rig }}, \underline{S}$ is the maximal $\breve{\mathcal{O}}\left[u^{ \pm 1}\right]$-split subtorus of $\underline{T}$, and $\underline{A}$ is the maximal $\mathcal{O}\left[u^{ \pm 1}\right]$-split subtorus of $\underline{T}$. In addition, the specialization of $(\underline{G}, \underline{A}, \underline{S}, \underline{P})$ by $u \mapsto \varpi$ is a rigidified reductive group

$$
\left(G_{0}, A_{0}, S_{0}, P_{0}\right):=(\underline{G}, \underline{A}, \underline{S}, \underline{P}) \otimes_{\mathcal{O}\left[u^{ \pm 1}\right]} F .
$$

By our construction, we have an isomorphism $G \simeq G_{0}$.

2.c.5. Similarly to Definition [1.7, let us define the groupoid $\operatorname{RG}\left(\mathcal{O}\left[u^{ \pm 1}\right]\right)$ of rigidified groups over $\mathcal{O}\left[u^{ \pm 1}\right]$. An object is a quadruple $(\underline{G}, \underline{A}, \underline{S}, \underline{P})$, where $\underline{G}$ is a connected reductive group over $\mathcal{O}\left[u^{ \pm 1}\right], \underline{A}$ is a maximal torus of $\underline{G}, \underline{S} \supset \underline{A}$ is a torus, which is maximal $\breve{\mathcal{O}}\left[u^{ \pm 1}\right]$-split, and $\underline{P}$ is a parabolic subgroup of $\underline{G}$ containing $\underline{M}=Z_{\underline{G}}(\underline{A})$ as a Levi factor, such that: (i) $(\underline{G}, \underline{A}, \underline{S}, \underline{P}) \otimes_{\mathcal{O}\left[u^{ \pm 1}\right]} F$ is a rigidified group as in Definition 1.7. (ii) there exists a pinned quasi-split form $\left(\underline{G}^{*}, \underline{T}^{*}, \underline{B}^{*}, \underline{e}^{*}\right)$ over $\mathcal{O}\left[u^{ \pm 1}\right]$ and an inner twist

$$
\underline{\psi}:(\underline{G}, \underline{S}, \underline{P}) \otimes_{\mathcal{O}\left[u^{ \pm 1}\right]} \breve{\mathcal{O}}\left[u^{ \pm 1}\right] \stackrel{\sim}{\rightarrow}\left(\underline{G}^{*}, \underline{S}^{*}, \underline{P}^{*}\right) \otimes_{\mathcal{O}\left[u^{ \pm 1}\right]} \breve{\mathcal{O}}\left[u^{ \pm 1}\right],
$$

where $\underline{S}^{*}$ is the maximal $\breve{\mathcal{O}}\left[u^{ \pm 1}\right]$-split subtorus of $\underline{T}^{*}$ and $\underline{P}^{*}$ is a parabolic subgroup of $\underline{G}^{*}$ containing $\underline{B}^{*}$.

Observe that an inner twist $\underline{\psi}$ defines a cocycle $\underline{c}=\psi \cdot \sigma(\psi)^{-1}$ with values in $\underline{N^{\prime *}}$, where as before $\underline{N}^{\prime *}$ is the normalizer of $\underline{T}^{*}$ in $\underline{M}^{\prime *}$, and $\underline{M}^{\prime *}$ is the standard Levi in $\underline{G}_{\text {ad }}^{*}$ given by $\underline{P}_{\text {ad }}^{*}$. 
By definition, we have the specialization functor $\operatorname{RG}\left(\mathcal{O}\left[u^{ \pm 1}\right]\right) \rightarrow \operatorname{RG}(F)$. Our construction shows that the essential image of this functor consists of all rigidified groups over $F$ that are split over $F^{t}$ (we denote this latter subgroupoid by $\operatorname{RG}\left(F^{t} / F\right)$ ). In fact, assuming Remark 2.1, it is easy to see that the isomorphism classes in $\operatorname{RG}\left(\mathcal{O}\left[u^{ \pm 1}\right]\right)$ biject to the isomorphism classes in $\operatorname{RG}\left(F^{t} / F\right)$.

Remark 2.2. Let $H$ be a Chevalley group over $\mathbb{Z}$. Recall that given a base scheme $S$, the set of isomorphism classes of forms $\mathcal{H}$ of $H$ over $S$ are classified by the (étale) cohomology set $\mathrm{H}^{1}(S, \underline{\mathrm{Aut}}(H))$. As every reductive group over $F$ admits a rigidification, a corollary of the above discussion is that the natural specialization map

$$
\mathrm{H}^{1}\left(\operatorname{Spec}\left(\mathcal{O}\left[u^{ \pm 1}\right]\right), \underline{\operatorname{Aut}}(H)\right) \rightarrow \mathrm{H}^{1}\left(F^{t} / F, \underline{\operatorname{Aut}}(H)\right)
$$

is surjective, where $\mathrm{H}^{1}\left(F^{t} / F\right.$, Aut $\left.(H)\right)$ denotes the set of isomorphism classes of forms of $H$ over $F$ that split over $F^{t}$.

It is natural to ask whether the map (2.14) is also injective. If this is the case, then each form of $H$ over $\mathcal{O}\left[u^{ \pm 1}\right]$, whose specialization along $u \mapsto \varpi$ is isomorphic to $G$, is isomorphic to $\underline{G}$. This would characterize $\underline{G}$ uniquely up to isomorphism. So far, we can prove a weaker result: If a form $\mathcal{H}$ of $H$ over $\mathcal{O}\left[u^{ \pm 1}\right]$ contains a maximal torus and splits over a finite Galois extension (as in 2.b.1) with Galois group $\Gamma$ of order prime to $p$, then $\mathcal{H}$ is isomorphic to $\underline{G}$ where $G=\mathcal{H} \otimes_{\mathcal{O}\left[u^{ \pm 1}\right]} F$ is the specialization of $\mathcal{H}$ along $u \mapsto \varpi$. This fact implies that (2.14) is injective when restricted to the subset which corresponds to such forms and provides, in the case that the Galois closure of the splitting field of $G$ has order prime to $p$, a characterization of the reductive group $\underline{G}$ as the unique up to isomorphism form of $H$ over $\mathcal{O}\left[u^{ \pm 1}\right]$ which contains a maximal torus, splits after a base change as above, and extends $G$. This result is obtained by using ideas of Pianzola and Gille; they have developed a theory of "loop reductive groups" over Laurent polynomial rings $K\left[u_{1}^{ \pm 1}, \ldots, u_{n}^{ \pm 1}\right]$ with $K$ a field (see for example [52], [11]). Essentially, these are reductive groups that afford a maximal torus over this base. The fibers $\underline{G} \otimes_{\mathcal{O}\left[u^{ \pm 1}\right]} F\left[u^{ \pm 1}\right]$, $\underline{G} \otimes_{\mathcal{O}\left[u^{ \pm 1}\right]} k\left[u^{ \pm 1}\right]$, of the groups $\underline{G}$ defined above are loop reductive groups in their sense. We intend to undertake a more comprehensive study of reductive groups over $\mathcal{O}\left[u^{ \pm 1}\right]$ and report on this in another paper.

\section{Parahoric group schemes over $\mathcal{O}[u]$}

In this chapter, we give our construction of the "parahoric" group schemes $\mathcal{G}$ over $\mathcal{O}[u]$ which are among the main tools of the paper. The main results are Theorem 3.1 and Corollary 3.2 which describes the properties of these group schemes.

3.a. Preliminaries. We start with some preliminaries on Bruhat-Tits buildings. Suppose that $K$ is a discretely valued quasi-local field ([40, 1.1.1]) with valuation ring $\mathcal{O}_{K}$ and with perfect residue field. If $G$ is a connected reductive group over $K$, we can consider the Bruhat-Tits building $\mathcal{B}(G, K)$. If $S$ is a maximal split torus of $G$, we denote by $\mathcal{A}(G, S, K)$ the corresponding apartment in $\mathcal{B}(G, K)$. 
Note that if $\hat{K}$ is the completion of $K$, the natural maps give identifications $\mathcal{A}(G, S, K)=$ $\mathcal{A}\left(G_{\hat{K}}, S_{\hat{K}}, \hat{K}\right), \mathcal{B}(G, K)=\mathcal{B}\left(G_{\hat{K}}, \hat{K}\right)$ ([40, Prop. 2.1.3]).

3.a.1. Suppose now that $\left(H, T_{H}, B_{H}, e\right)$ is a pinned Chevalley group scheme $H$ over $\mathbb{Z}$ and consider $H_{K}=H \otimes_{\mathbb{Z}} K$ and $T_{K}=T_{H} \otimes_{\mathbb{Z}} K$ over the field $K$. We will sometimes omit the subscripts when the choice of the field $K$ is clear. After fixing an identification of the value groups $v\left(K^{*}\right)=\mathbb{Z}$, we can identify the apartments $\mathcal{A}\left(H, T_{H}, K\right) \subset \mathcal{B}(H, K)$ for all such fields $K$. In particular, we can identify $\mathcal{A}\left(H, T_{H}, \kappa((u))\right)$ for $\kappa=F, k$ and also $\mathcal{A}\left(H, T_{H}, F\right)$ such that, under this identification, the hyperspecial points of $\mathcal{A}\left(H, T_{H}, K\right)$ with stabilizer $H\left(\mathcal{O}_{K}\right)$ correspond to each other. Observe that we can also identify the Iwahori-Weyl groups $\widetilde{W}=N_{H}\left(T_{H}\right)(K) / T_{H}\left(\mathcal{O}_{K}\right)$ for different $K$ canonically such that the above identification of the apartments is compatible with the action of $\widetilde{W}$.

3.a.2. The above generalizes to the quasi-split case as follows. Let $\left(\underline{G}^{*}, \underline{T}^{*}, \underline{B}^{*}, \underline{e}^{*}\right)$ be a pinned quasi-split group over $\mathcal{O}\left[u^{ \pm 1}\right]$. Let $\underline{S}^{*}$ be the maximal $\breve{\mathcal{O}}\left[u^{ \pm 1}\right]$-split subtorus of $\underline{T}^{*}$ and $\underline{A}^{*}$ be the maximal $\mathcal{O}\left[u^{ \pm 1}\right]$-split torus of $\underline{T}^{*}$. Then as the centralizer of $\underline{S}^{*}$ is $\underline{T}^{*}$, we see that $\underline{S}_{\kappa^{\prime}((u))}^{*}:=\underline{S}^{*} \otimes_{\mathcal{O}\left[u^{ \pm 1}\right]} \kappa^{\prime}((u))$ is a maximal $\kappa^{\prime}((u))$-split torus of $\underline{G}_{\kappa^{\prime}((u))}^{*}:=$ $\underline{G}^{*} \otimes_{\mathcal{O}\left[u^{ \pm 1}\right]} \kappa^{\prime}((u))$ for $\kappa^{\prime}=\bar{k}, \breve{F}$. Similarly, $\underline{S}_{\breve{F}}^{*}:=\underline{S}^{*} \otimes_{\mathcal{O}\left[u^{ \pm 1}\right]} \breve{F}$ is a maximal $\breve{F}$-split torus of $\underline{G}_{\breve{F}}^{*}:=\underline{G}^{*} \otimes_{\mathcal{O}[u \pm 1]} \breve{F}$. We have canonical identifications

$$
\mathcal{A}\left(\underline{G}_{\kappa^{\prime}((u))}^{*}, \underline{S}_{\kappa^{\prime}((u))}^{*}, \kappa^{\prime}((u))\right)=\mathcal{A}\left(\underline{G}_{\breve{F}}^{*}, \underline{S}_{\breve{F}}^{*}, \breve{F}\right) .
$$

Indeed, these apartments are identified with $\mathcal{A}\left(H, T_{H}, K\right)^{\gamma}$ (using the tameness assumption, as in [7], or [39, Ch. IV, §10], see also [53]), where $K=\kappa^{\prime}((u))$ or $\breve{F}$, and $\gamma$ is a generator of the inertia subgroup. Similary, we can identify the Iwahori-Weyl group for $\left(\underline{G}_{K}^{*}, \underline{S}_{K}^{*}\right)$ canonically and compatibly with the identification of the apartments.

3.a.3. We also have the following further generalization. Let $(\underline{G}, \underline{A}, \underline{S}, \underline{P})$ be a rigidified group over $\mathcal{O}\left[u^{ \pm 1}\right]$ as defined in 92. .c.5. Then $\underline{A}_{F}$ is a maximal $F$-split torus of $\underline{G}_{F}$. Let $x \in \mathcal{A}\left(\underline{G}_{F}, \underline{A}_{F}, F\right) \subset \mathcal{B}\left(\underline{G}_{F}, F\right)$ be a point in the apartment. The identification (3.1) induces

$$
\begin{aligned}
\mathcal{A}\left(\underline{G}_{F}, \underline{A}_{F}, F\right) & =\mathcal{A}\left(\underline{G}_{\breve{F}}, \underline{S}_{\breve{F}}, \breve{F}\right)^{\sigma}=\mathcal{A}\left(\underline{G}_{\breve{F}}^{*}, \underline{S}_{\breve{F}}^{*}, \breve{F}\right)^{\operatorname{Int}(\mathrm{g}) \sigma} \\
= & \mathcal{A}\left(\underline{G}_{\kappa^{\prime}((u))}^{*}, \underline{S}_{\kappa^{\prime}((u))}^{*}, \kappa^{\prime}((u))\right)^{\operatorname{Int}(\mathrm{g}) \sigma}=\mathcal{A}\left(\underline{G}_{\kappa^{\prime}((u))}, \underline{S}_{\kappa^{\prime}((u))}, \kappa^{\prime}((u))\right)^{\sigma},
\end{aligned}
$$

and therefore we obtain $x_{\kappa((u))} \in \mathcal{A}\left(\underline{G}_{\kappa^{\prime}((u))}, \underline{S}_{\kappa^{\prime}((u))}, \kappa^{\prime}((u))\right)^{\sigma}$. Therefore, using again $\mathcal{B}\left(\underline{G}_{\kappa^{\prime}((u))}, \kappa^{\prime}((u))\right)^{\sigma}=\mathcal{B}\left(\underline{G}_{\kappa((u))}, \kappa((u))\right)$, we see that $x_{\kappa((u))}$ can be regarded as a point in the building $\mathcal{B}\left(\underline{G}_{\kappa((u))}, \kappa((u))\right)$.

3.b. The main construction. We now state the main result of this section. Let $(\underline{G}, \underline{A}, \underline{S}, \underline{P})$ be a rigidified group over $\mathcal{O}\left[u^{ \pm 1}\right]$ as defined in 92. c.5. Let $x \in \mathcal{A}\left(\underline{G}_{F}, \underline{A}_{F}, F\right)$. By 3.a.3, we also obtain from $x$ points $x_{\kappa((u))}$ in $\mathcal{B}\left(\underline{G}_{\kappa((u))}, \kappa((u))\right)$ for $\kappa=F$ or $k$.

Theorem 3.1. There is a unique smooth, affine group scheme $\mathcal{G}=\mathcal{G}_{x} \rightarrow \mathbb{A}_{\mathcal{O}}^{1}=$ $\operatorname{Spec}(\mathcal{O}[u])$ (called a Bruhat-Tits group scheme for $\underline{G}$ ) with connected fibers and with the following properties: 
1) The group scheme $\mathcal{G}_{\mid \mathcal{O}\left[u, u^{-1}\right]}$ is the group scheme $\underline{G}$.

2) The base change of $\mathcal{G}$ under $\operatorname{Spec}(\mathcal{O}) \rightarrow \mathbb{A}_{\mathcal{O}}^{1}$ given by $u \mapsto \varpi$ is the parahoric group scheme $\mathcal{P}_{x}$ for $\underline{G}_{F}$ (as defined in [7]).

3) The base change of $\mathcal{G}$ under $\operatorname{Spec}(\kappa[[u]]) \rightarrow \mathbb{A}_{\mathcal{O}}^{1}$ given by $\mathcal{O}[u] \rightarrow \kappa[[u]]$ is the parahoric group scheme $\mathcal{P}_{x_{\kappa((u))}}$ for $\underline{G}_{\kappa((u))}$.

3.b.1. We first prove the uniqueness statement in Theorem 3.1. Recall that the parahoric group scheme $\mathcal{P}_{x}$ is a group scheme (smooth, affine, connected) over $\operatorname{Spec}(\mathcal{O})$ with generic fiber $\underline{G}_{F}$ such that $\mathcal{P}_{x}(\mathcal{O}) \subset \underline{G}(F)$ is the connected stabilizer of $x$. Similarly for $\mathcal{P}_{x_{\kappa((u))}}$.

We will show that if $\mathcal{G}^{\prime}$ is a smooth affine connected group scheme over $\mathcal{O}[u]$ with $\mathcal{G}\left[u^{-1}\right]=\underline{G}$ and which is such that $\mathcal{G}^{\prime}(F[[u]]) \subset \underline{G}(F((u)))$ is the connected stabilizer of $x_{F((u))}$ in the building of $\underline{G}_{F((u))}$ then $\mathcal{G}^{\prime}=\mathcal{G}_{x}$. In particular, this will show the uniqueness. To see this set $\mathcal{G}_{x}=\operatorname{Spec}(B), \mathcal{G}^{\prime}=\operatorname{Spec}\left(B^{\prime}\right)$. Our assumptions imply $B\left[u^{-1}\right]=B^{\prime}\left[u^{-1}\right]$ and that $\mathcal{G}^{\prime}(F[[u]])=\mathcal{G}_{x}(F[[u]])$. Since $F$ is infinite and perfect, $F[[u]]$ is henselian and both the group schemes $\mathcal{G}^{\prime}$ and $\mathcal{G}_{x}$ are smooth, condition (ET 2) of [7, 1.7.2] is satisfied; hence the second identity implies that $B^{\prime} \otimes_{\mathcal{O}[u]} F[[u]]=B \otimes_{\mathcal{O}[u]} F[[u]]$. Observe now that since $B$ is smooth over $\mathcal{O}[u]$,

$$
B=B\left[u^{-1}\right] \cap\left(B \otimes_{\mathcal{O}[u]} F[[u]]\right)
$$

and similarly for $B^{\prime}$. Hence $B=B^{\prime}$ which gives $\mathcal{G}^{\prime}=\mathcal{G}_{x}$. This actually shows that $\mathcal{G}_{x}$ only depends on $\underline{G}$ and $x_{F((u))}$.

3.b.2. Here we show the existence part of Theorem 3.1.

a) First suppose that $\underline{G}=H \otimes_{\mathbb{Z}} \mathcal{O}\left[u^{ \pm 1}\right]$ is split; we will then consider more general convex subsets of the apartment (and not just points). Denote by $\Phi=\Phi\left(H, T_{H}\right)$ the corresponding root system. The pinning $\left(H, T_{H}, B_{H}, e\right)$ gives a hyperspecial vertex $x_{0}$ of the apartment $\mathcal{A}\left(H, T_{H}, K\right)$ of $T_{H}$. This determines a filtration $\left\{U_{a}(K)_{x_{0}, r}\right\}_{r \in \mathbb{R}}$ of the corresponding root subgroups for any local field $K$. Let $f: \Phi \rightarrow \mathbb{R}$ be a concave function; there is an associated convex subset $\Omega=\Omega_{f}$ of the apartment $A$ given by

$$
\Omega=\left\{x \in A \mid a \cdot\left(x-x_{0}\right)+f(a) \geq 0\right\} .
$$

Conversely, to a convex bounded subset $\Omega \subset A$, we can associate the concave function $f_{\Omega}: \Phi \rightarrow \mathbb{R}$ given by

$$
f_{\Omega}(a)=\inf \left\{\lambda \in \mathbb{R} \mid a \cdot\left(x-x_{0}\right)+\lambda \geq 0, \forall x \in \Omega\right\}
$$

Notice that $x_{0} \in \Omega_{f}$ if and only if $f \geq 0$. Now denote by $H(K)_{x_{0}, f}$ the subgroup of $H(K)$ generated by $U_{a}(K)_{x_{0}, f(a)}$ and $T_{H}\left(\mathcal{O}_{K}\right)$. By [7], there is an associated connected affine smooth group scheme $\mathcal{P}_{x_{0}, f, K} \operatorname{over} \operatorname{Spec}\left(\mathcal{O}_{K}\right)$. This only depends on $\Omega$ and we can denote it by $\mathcal{P}_{\Omega}$.

We will start by explaining how to construct a smooth affine group scheme $\mathcal{G}_{x_{0}, f}$ over $\mathcal{O}[u]$ that lifts $\mathcal{P}_{x_{0}, f, K}$ for $K=F$ and $K=k((u))$ as it is required in the statement of the theorem. We first consider the additive group schemes $\mathcal{U}_{a, x_{0}, f}=u^{\langle f(a)\rangle} U_{a} \otimes_{\mathbb{Z}} \mathcal{O}[u] \simeq$ $\mathbb{G}_{a} \otimes_{\mathbb{Z}} \mathcal{O}[u]$ over $\mathcal{O}[u]$ and the torus $\mathcal{T}=T_{H} \otimes_{\mathbb{Z}} \mathcal{O}[u]$. (Here $\langle r\rangle$ is the smallest integer that is larger or equal to $r)$. Notice here that by definition $u U_{a} \otimes_{\mathbb{Z}} \mathcal{O}[u]=\operatorname{Spec}(\mathcal{O}[u, x / u])$ is 
the dilatation of $U_{a} \otimes_{\mathbb{Z}} \mathcal{O}[u] \simeq \mathbb{G}_{a} \otimes_{\mathbb{Z}} \mathcal{O}[u]$ along the zero section $\operatorname{Spec}(\mathcal{O}) \rightarrow \mathbb{G}_{a} \otimes_{\mathbb{Z}} \mathcal{O} \hookrightarrow$ $\mathbb{G}_{a} \otimes_{\mathbb{Z}} \mathcal{O}[u]$ over $u=0$. Similarly, $u^{i} U_{a} \otimes_{\mathbb{Z}} \mathcal{O}[u]=\operatorname{Spec}\left(\mathcal{O}\left[u, u^{-i} x\right]\right)$. Since $f$ is concave, these group schemes give as in [7, 3.1.1], or [39], schematic root data over $\mathcal{O}[u]$. (In particular, see [7, 3.2] for this split case.) By using [7] (3.9.4 together with 2.2.10) we obtain a (smooth) group scheme $\mathcal{G}_{x_{0}, f}$ over $\mathcal{O}[u]$ which has a fiberwise dense open subscheme given by

$$
\mathcal{V}_{x_{0}, f}=\prod_{a \in \Phi^{-}} \mathcal{U}_{a, x_{0}, f} \times \mathcal{T} \times \prod_{a \in \Phi^{+}} \mathcal{U}_{a, x_{0}, f} .
$$

By [7, 1.2.13, 1.2.14] this group scheme is uniquely determined from the schematic root data. By [7] or [39] the group schemes $\mathcal{G}_{x_{0}, f, K}$ are also given by the schematic root data obtained by base changing the schematic root data on $\mathcal{V}_{x_{0}, f}$ via $\mathcal{O}[u] \rightarrow \mathcal{O}_{K}$. This implies that $\mathcal{G}_{x_{0}, f}$ specializes to $\mathcal{P}_{x_{0}, f}$ as in (2) and (3). Property (1) also follows easily. It remains to show that $\mathcal{G}_{x_{0}, f}$ is affine.

(I) We first assume that $\Omega$ contains the hyperspecial vertex $x_{0}$, i.e we can take $f \geq 0$.

When $f=0, \mathcal{G}_{x_{0}, f} \simeq H \otimes_{\mathbb{Z}} \mathcal{O}[u]$ which is affine. We will build on this, showing that, in general, $\mathcal{G}_{x_{0}, f}$ can be obtained from $\mathcal{G}_{x_{0}, 0}$ by a series of dilatations. This follows an argument of $\mathrm{Yu}([74])$ and provides an alternative construction of the group schemes. Since such dilatations are affine, we will obtain the desired conclusion.

(i) Assume first that $0 \leq f \leq 1$. Consider the parabolic subgroup $P_{f} \subset H$ over $\mathbb{Z}$ containing $T=T_{H}$ which corresponds to the set of roots $a \in \Phi$ with $f(a)=0$ : For any field $k, P_{f}(k)$ is generated by $T(k)$ and $U_{a}(k)$ with $f(a)=0$. In this case, we can consider the dilatation of $H \otimes_{\mathbb{Z}} \mathcal{O}[u]$ along the closed subgroup scheme given by $P_{f} \otimes_{\mathbb{Z}} \mathcal{O} \hookrightarrow H \otimes_{\mathbb{Z}} \mathcal{O}[u]$ over $u=0$ : Suppose that $H=\operatorname{Spec}(A)$ and $P_{f}=\operatorname{Spec}(A / I)$. Set $J=\mathcal{O} \cdot I+(u)$ for the ideal generated by $I$ and $u$ in $A \otimes \mathcal{O}[u]$. Then we consider

$$
B=(A \otimes \mathcal{O}[u])\left[j u^{-1} \mid j \in J\right] \subset A \otimes \mathcal{O}\left[u, u^{-1}\right]
$$

and set

$$
\mathcal{H}_{x_{0}, f}=\operatorname{Spec}(B)
$$

(We refer the reader to [73] for basic properties of dilatations of group schemes.) If $R$ is an $\mathcal{O}[u]$-algebra we set $\bar{R}=R /(u)=R \otimes_{\mathcal{O}[u]} \mathcal{O}$ and we denote by $\bar{h} \in H(\bar{R})$ the reduction of $h \in H(R)$. Then $\mathcal{H}_{x_{0}, f}$ is the unique, up to isomorphism, group scheme with $u$ not a zero divisor in its structure sheaf, that supports a homomorphism $\Psi: \mathcal{H}_{x_{0}, f} \rightarrow H \otimes_{\mathbb{Z}} \mathcal{O}[u]$ with the following properties:

- $\Psi$ is an isomorphism away from $u=0$,

- for any $\mathcal{O}[u]$-algebra $R$ with $u$ not a zero divisor in $R, \Psi$ identifies $\mathcal{H}_{x_{0}, f}(R)$ with the subset $\left\{h \in H(R) \mid \bar{h} \in P_{f}(\bar{R})\right\}$ of $H(R)$.

We can see that $\mathcal{H}_{x_{0}, f}$ has connected fibers and is given by the same group germ $\mathcal{V}_{x_{0}, f}$ as $\mathcal{G}_{x_{0}, f}$. Therefore, $\mathcal{G}_{x_{0}, f} \simeq \mathcal{H}_{x_{0}, f}=\operatorname{Spec}(B)$ and is also affine.

(ii) In general, we can find a finite sequence $0=t_{0}<t_{1}<\cdots<t_{n}=1$ such that if $f_{i}=t_{i} \cdot f$, we have $f_{n}=f$, and $f_{i+1} \leq f_{i}+1$. We will use induction on $n$. The case $n=1$ is given by (i) above. Note that by the construction of [7, 3.9.4] we have: 
a) There are closed group scheme embeddings $\mathcal{U}_{a, i} \hookrightarrow \mathcal{H}_{i}$ and $\mathcal{T} \hookrightarrow \mathcal{H}_{i}$ that extend the standard embeddings of the root subgroups and the maximal torus over $\mathcal{O}\left[u, u^{-1}\right]$. (Here and in the rest of the proof, for simplicity, we omit some subscripts and write for example $\mathcal{U}_{a, i}, \mathcal{H}_{i}$, instead of $\mathcal{U}_{a, x_{0}, f_{i}}, \mathcal{H}_{x_{0}, f_{i}}$.)

b) The embeddings in (a) combined with the multiplication morphism induce an open immersion

$$
j: \mathcal{V}_{i}=\prod_{a \in \Phi^{-}} \mathcal{U}_{a, i} \times \mathcal{T} \times \prod_{a \in \Phi^{+}} \mathcal{U}_{a, i} \hookrightarrow \mathcal{G}_{i}
$$

onto a fiberwise dense open subscheme of $\mathcal{G}_{i}$ which makes the schematic root data $\mathcal{D}_{i}=$ $\left(\mathcal{T},\left(\mathcal{U}_{a, i}\right)_{a \in \Phi}\right)$ compatible with $\mathcal{H}_{i}$ (in the sense of [7], 3.1.3).

c) The group scheme multiplication morphism

$$
\mathcal{V}_{i} \times \mathcal{V}_{i} \rightarrow \mathcal{G}_{i}
$$

is surjective on each fiber.

Our induction hypothesis is: The smooth group scheme $\mathcal{G}_{n}:=\mathcal{G}_{x_{0}, f_{n}}$ is affine and supports a group scheme extension

$$
1 \rightarrow R_{n} \rightarrow \overline{\mathcal{G}}_{n} \rightarrow \overline{\mathcal{G}}_{n}^{\text {red }} \rightarrow 1
$$

of a (connected) reductive group scheme by a smooth affine unipotent group scheme (both over $\mathcal{O})$. Here the bar indicates base change via $\mathcal{O}[u] \rightarrow \mathcal{O}$ given by $u \mapsto 0$. The case $n=1$ follows from (i) above. Then, $\overline{\mathcal{G}}_{1}^{\text {red }}$ is the Levi component of the parabolic $P$.

Let us consider $\mathcal{G}_{n+1}$. There is a natural morphism $\mathcal{U}_{a, x_{0}, f_{n+1}} \rightarrow \mathcal{U}_{a, x_{0}, f_{n}}$. This is the identity when $f_{n+1}(a)=f_{n}(a)$ and is given by dilatation of the zero section over $u=0$ when $f_{n+1}(a)>f_{n}(a)$. These morphisms combine to give $f_{n}: \mathcal{V}_{n+1} \rightarrow \mathcal{V}_{n} \subset \mathcal{G}_{n}$. Since $\mathcal{G}_{n+1}\left[u^{-1}\right]=\mathcal{G}_{n}\left[u^{-1}\right]$, by [7, 1.2.13] there is a unique group scheme homomorphism $\tilde{f}_{n}: \mathcal{G}_{n+1} \rightarrow \mathcal{G}_{n}$ that extends $f_{n}$.

We will now show that $\tilde{f}_{n}$ identifies $\mathcal{G}_{n+1}$ with a dilatation of $\mathcal{G}_{n}$. Consider the (settheoretic image) $Q_{n}$ of $\overline{\mathcal{G}}_{n+1} \rightarrow \overline{\mathcal{G}}_{n}$ which is a constructible set. We will show that $Q_{n}$ is closed in $\overline{\mathcal{G}}_{n}$ and is underlying a smooth group scheme which we will also denote by $Q_{n}$. (This will imply that $\mathcal{G}_{n+1} \rightarrow \mathcal{G}_{n}$ factors through $\mathcal{G}_{n+1} \rightarrow \mathcal{G}_{n}^{\prime} \rightarrow \mathcal{G}_{n}$ where $\mathcal{G}_{n}^{\prime} \rightarrow \mathcal{G}_{n}$ is the dilatation of $\mathcal{G}_{n}$ along $Q_{n} \subset \overline{\mathcal{G}}_{n}$.) Using property (c) of $\mathcal{G}_{n+1}$, we see that the image $Q_{n}$ can be identified with the image of $\overline{\mathcal{V}}_{n+1} \times \overline{\mathcal{V}}_{n+1}$ under the product map in $\overline{\mathcal{G}}_{n}$. By construction, we have morphisms $\overline{\mathcal{U}}_{a, n+1} \rightarrow Q_{n}$. Suppose $\kappa$ is either $F$ or $k$. Denote by $V_{a, n}(\kappa)$ the image of the corresponding $\overline{\mathcal{U}}_{a, n+1}(\kappa) \rightarrow Q_{n}(\kappa)$. Then by the above, we see that the $\kappa$-valued points $Q_{n}(\kappa)$ is the subgroup of $\overline{\mathcal{G}_{n}}(\kappa)$ generated by the groups $V_{a, n}(\kappa)$ and $\overline{\mathcal{T}}(\kappa)$. By the argument in [74, 8.3.2], we see that the fibers of $Q_{n}$ over $\kappa=k$ and $F$, are closed in the corresponding fibers of $\mathcal{G}_{n}$. Now consider the extension (3.3). In this, $\overline{\mathcal{G}}_{n}^{\text {red }}$ contains the maximal torus $\overline{\mathcal{T}}$ and it corresponds to the root datum given by $\left(\mathbb{X}^{\bullet}(\overline{\mathcal{T}}), \Phi_{f_{n}}, \mathbb{X}_{\bullet}(\overline{\mathcal{T}}), \Phi_{f_{n}}^{\vee}\right)$ ) (with $\left.\Phi_{f}=\{a \in \Phi \mid f(a)+f(-a)=0\}\right)$. On the other hand, as a scheme, $R_{n}$ is an affine space:

$$
R_{n}=\prod_{\left\{a \in \Phi \mid f_{n}(a)+f_{n}(-a)>0\right\}} \overline{\mathcal{U}}_{a, n} .
$$


We now consider the Zariski closure $\tilde{Q}_{n}$ of the generic fiber $Q_{n}(F)$ in $\overline{\mathcal{G}}_{n}$. This agrees with the Zariski closure of $\overline{\mathcal{V}}_{n+1} \hookrightarrow \overline{\mathcal{G}}_{n}$. We have of course $Q_{n} \subset \tilde{Q}_{n}$. We can see that $\tilde{Q}_{n} \subset \overline{\mathcal{G}}_{n}$ maps in $\overline{\mathcal{G}}_{n}^{\text {red }}$ onto the closed parabolic subgroup scheme $P_{n}$ of $\overline{\mathcal{G}}_{n}^{\text {red }}$ generated by $\overline{\mathcal{T}}$ and $\overline{\mathcal{U}}_{a, n}$ with $f_{n+1}(a)=f_{n}(a)=0$. On the other hand, the intersection of $\tilde{Q}_{n}$ with $R_{n}$ can be identified as the closed subscheme of $R_{n}=\prod_{a, f_{n}(a)+f_{n}(-a)>0} \overline{\mathcal{U}}_{a, n}$ given by the product of those $\overline{\mathcal{U}}_{a, n}$ for which $f_{n+1}(a)=f_{n}(a)$ (with $f_{n}(a)+f_{n}(-a)>0$ ). This and the above allows us to conclude that $\tilde{Q}_{n}$ is an affine fibration over $P_{n}$ and so all fibers of $\tilde{Q}_{n}$ are geometrically connected. It follows that $Q_{n}=\tilde{Q}_{n}$ and so $Q_{n}$ is a smooth closed subgroup scheme of $\overline{\mathcal{G}}_{n}$. We can see following the argument in [74, 8.3.3], that we have

$$
Q_{n} \cap \overline{\mathcal{V}}_{n}=\operatorname{Im}\left(\overline{\mathcal{V}}_{n+1} \rightarrow \overline{\mathcal{V}}_{n}\right)
$$

As above $\mathcal{G}_{n+1} \rightarrow \mathcal{G}_{n}$ factors $\mathcal{G}_{n+1} \rightarrow \mathcal{G}_{n}^{\prime} \rightarrow \mathcal{G}_{n}$, where $\mathcal{G}_{n}^{\prime}$ is the dilatation of $\mathcal{G}_{n}$ along $Q_{n} \subset \overline{\mathcal{G}}_{n}$. Then $\mathcal{G}_{n}^{\prime}$ is a smooth affine group scheme over $\operatorname{Spec}(\mathcal{O}[u])$ with connected fibers. Observe that $\mathcal{U}_{a, f_{n+1}}$ is by definition isomorphic to the dilatation of $\mathcal{U}_{a, f_{n}}$ along the image of the morphism $\overline{\mathcal{U}}_{a, f_{n+1}} \rightarrow \overline{\mathcal{U}}_{a, f_{n}}$. As a result, the dilatation of $\mathcal{V}_{n}$ along the image of $\overline{\mathcal{V}}_{n+1} \rightarrow \overline{\mathcal{V}}_{n}$ is isomorphic to $\overline{\mathcal{V}}_{n+1}$. It now follows from the functoriality of the dilatation construction and (3.4) that the dilatation $\mathcal{G}_{n}^{\prime}$ of $\mathcal{G}_{n}$ along $Q_{n}$ has an open subscheme isomorphic to $\mathcal{V}_{n+1} \subset \mathcal{G}_{n+1}$. Since $\mathcal{V}_{n+1}$ is fiberwise dense in $\mathcal{G}_{n+1}$ it follows that $\mathcal{G}_{n+1}=\mathcal{G}_{n}^{\prime}$ and hence $\mathcal{G}_{n+1}$ is also affine. The rest of the induction hypothesis for $\mathcal{G}_{n+1}$ also follow. Again, $\overline{\mathcal{G}}_{n+1}^{\text {red }}$ is the Levi component of the parabolic of $\overline{\mathcal{G}}_{n}^{\text {red }}$ that corresponds to $a \in \Phi_{f_{n}}$ for which $f_{n+1}(a)=f_{n}(a)$.

II) We continue to assume that $\underline{G}=H \otimes_{\mathbb{Z}} \mathcal{O}\left[u^{ \pm 1}\right]$ is split but now we consider the general case in which $\Omega=\Omega_{f}$ does not contain $x_{0}$. The argument in the proof of [18, Lemma 2.2] (see also [4]) implies that there is an integer $\delta \geq 1$ such that the subset $\Omega_{\delta \cdot f}$ of the apartment contains a hyperspecial vertex $x_{0}^{\prime}$ which is the translate $x_{0}^{\prime}=x_{0}+t$ of $x_{0}$ by an element $t \in \mathbb{X}_{\bullet}(T)$. Consider the homomorphism $\mathcal{O}[u] \rightarrow \mathcal{O}[v]$ given by $u \mapsto v^{\delta}$. Our previous arguments allow us to construct, using successive dilatations of $t\left(H \otimes_{\mathbb{Z}} \mathcal{O}[v]\right) t^{-1} \simeq H \otimes_{\mathbb{Z}} \mathcal{O}[v]$, a smooth affine group scheme $\mathcal{G}_{\Omega}^{\prime}=\mathcal{G}_{x_{0}^{\prime}, f^{\prime}}$ over $\mathcal{O}[v]$. (Here $f^{\prime}=\delta \cdot f+t$ which is positive.) We can however see that base changing the schematic root data for $\mathcal{G}_{\Omega}$ by $\mathcal{O}[u] \rightarrow \mathcal{O}[v]$ gives schematic root data for $\mathcal{G}_{\Omega}^{\prime}$. As above, using [7, $1.2 .13,1.2 .14]$, this implies that $\mathcal{G}_{\Omega} \otimes_{\mathcal{O}[u]} \mathcal{O}[v] \simeq \mathcal{G}_{\Omega}^{\prime}$. By faithful flat descent, $\mathcal{G}_{\Omega}$ is then affine.

b) We now consider the more general case of a quasi-split group $\underline{G}$ that splits over an extension $\mathcal{O}_{0}\left[v^{ \pm 1}\right] / \mathcal{O}\left[u^{ \pm 1}\right]$ as in 2.b.1. In particular, $\mathcal{O}$ has residue field $k=\mathbb{F}_{q}$, $u \mapsto v^{e}$, and $\Gamma^{t}$ acts on $\tilde{\mathcal{O}}_{0}[v]$ by $\gamma_{0}(v)=\zeta \cdot v, \sigma\left(\sum a_{i}^{\prime} v^{i}\right)=\sum_{i} \sigma\left(a_{i}^{\prime}\right) v^{i}$, with invariants $\tilde{\mathcal{O}}_{0}[v]^{\Gamma}=\mathcal{O}[u]$. Let $\tilde{F} / F$ be the base change of $\mathcal{O}_{0}\left[v^{ \pm 1}\right] / \mathcal{O}\left[u^{ \pm 1}\right]$ along $u=\varpi$, with the maximal unramified extension $\tilde{F}_{0} / F$ of degree $r$ and $e=\left[\tilde{F}: \tilde{F}_{0}\right]$.

As $\underline{G}$ is quasi-split, $\underline{G}=\underline{G}^{*}$. We consider $\Omega \subset \mathcal{A}\left(\underline{G}_{F}, \underline{A}_{F}, F\right)=\mathcal{A}\left(H, T_{H}, \tilde{F}\right)^{\Gamma} \subset$ $\mathcal{A}\left(H, T_{H}, \tilde{F}\right)$ and let $\mathcal{H}_{\Omega}$ be the smooth affine group scheme over $\mathbb{A}_{\tilde{\mathcal{O}}_{0}}^{1}=\operatorname{Spec}\left(\tilde{\mathcal{O}}_{0}[v]\right)$ constructed for the split group $H$ as in (a). The group $\Gamma$ acts on the apartment $\mathcal{A}\left(H, T_{H}, \tilde{F}\right)$ via its action on $H$ and $\tilde{F}$. Since $\Omega$ is fixed by $\Gamma$, we can see that $\mathcal{H}_{\Omega}$ supports a $\Gamma$ action that lifts the action on $\Gamma$ on $\mathbb{A}_{\tilde{\mathcal{O}}_{0}}^{1}$. Notice that the Weil restriction of scalars 
$\operatorname{Res}_{\tilde{\mathcal{O}}_{0}[v] / \mathcal{O}[u]} \mathcal{H}_{\Omega}$ is also a smooth affine group scheme over $\mathcal{O}[u]([7$, [15, 2.2]). By the above and [15, 2.4], this supports a $\Gamma$-action over $\operatorname{Spec}(\mathcal{O}[u])$; the inertia groups for this action are always subgroups of $\left\langle\gamma_{0}\right\rangle$. Since, $\gamma_{0}$ has order prime to $p$, by [15, 3.4], the fixed point scheme $\mathcal{G}_{\Omega}^{\prime}=\left(\operatorname{Res}_{\tilde{\mathcal{O}}_{0}[v] / \mathcal{O}[u]} \mathcal{H}_{\Omega}\right)^{\Gamma}$ is a smooth closed subscheme of the smooth affine $\operatorname{Res}_{\tilde{\mathcal{O}}_{0}[v] / \mathcal{O}[u]} \mathcal{H}_{\Omega}$. Hence, it is also flat over $\mathcal{O}[u]$. Consider the base change $\mathcal{G}_{\Omega}^{\prime} \otimes_{\mathcal{O}[u]} \mathcal{O}$ by $u \mapsto 0$. Since this is also smooth over $\mathcal{O}$, it is the disjoint union

$$
\mathcal{G}_{\Omega}^{\prime} \otimes_{\mathcal{O}[u]} \mathcal{O}=Z^{0} \sqcup\left(\sqcup_{i} Z_{i}\right)
$$

of its smooth irreducible components where $Z^{0}$ contains the identity section. By flatness of $\mathcal{G}_{\Omega}^{\prime} \rightarrow \mathbb{A}_{\mathcal{O}}^{1}$, all the components $Z^{0}, Z_{i}$ are divisors in $\mathcal{G}_{\Omega}^{\prime}$. We will set

$$
\mathcal{G}_{\Omega}^{\prime \prime}=\mathcal{G}_{\Omega}^{\prime}-\sqcup_{i} Z_{i}
$$

(i.e the complement of the union of those components that do not contain the identity.) Observe that $\mathcal{G}_{\Omega}^{\prime \prime}$ is affine since it can also be obtained as the dilatation of the affine $\mathcal{G}_{\Omega}^{\prime}$ along the affine and smooth closed subscheme $Z^{0}$ of its fiber over $u=0$. We will now show that $\mathcal{G}_{\Omega}^{\prime \prime}$ is the connected component $\mathcal{G}_{\Omega}^{\prime 0}$ of $\mathcal{G}_{\Omega}^{\prime}$. By [7, 1.2.12] we have to show that each fiber of $\mathcal{G}_{\Omega}^{\prime \prime} \rightarrow \mathbb{A}_{\mathcal{O}}^{1}$ is connected. First observe that the geometric fibers at points where $u \neq 0$ are isomorphic to the split form $H$ and so they are connected. By construction, $\mathcal{G}_{\Omega}^{\prime \prime} \otimes_{\mathcal{O}[u]} \mathcal{O}=Z^{0}$; therefore the fiber $\mathcal{G}_{\Omega}^{\prime \prime} \otimes_{\mathcal{O}[u]} F$ is connected and is the connected component of $\mathcal{G}_{\Omega}^{\prime} \otimes_{\mathcal{O}[u]} F$. In general for $\kappa=F$ or $k$, let $\kappa^{\prime}$ be $\tilde{F}_{0}$, resp. the residue field of $\tilde{\mathcal{O}}_{0}$. We can consider the fiber over $\mathcal{O}[u] \rightarrow \mathcal{O} \rightarrow \kappa^{\prime}$

$$
\mathcal{G}_{\Omega}^{\prime} \otimes_{\mathcal{O}[u]} \kappa^{\prime}=\left(\operatorname{Res}_{\mathcal{O}^{\prime}[v] / \mathcal{O}[u]}\left(\mathcal{H}_{\Omega}\right) \otimes_{\mathcal{O}[u]} \kappa^{\prime}\right)^{\Gamma} .
$$

Since $\Gamma$ is an extension of $\operatorname{Gal}\left(\kappa^{\prime} / \kappa\right)$ by $\left\langle\gamma_{0}\right\rangle$ this is

$$
\left(\operatorname{Res}_{\kappa^{\prime}[v] /\left(v^{e}\right) / \kappa^{\prime}}\left(\mathcal{H}_{\Omega} \otimes_{\tilde{\mathcal{O}}_{0}[v]} \kappa^{\prime}[v] /\left(v^{e}\right)\right)\right)^{\gamma_{0}} .
$$

We have

$$
1 \rightarrow U \rightarrow \operatorname{Res}_{\kappa^{\prime}[v] /\left(v^{e}\right) / \kappa^{\prime}}\left(\mathcal{H}_{\Omega} \otimes_{\tilde{\mathcal{O}}_{0}[v]} \kappa^{\prime}[v] /\left(v^{e}\right)\right) \rightarrow \overline{\mathcal{H}}_{\Omega, \kappa^{\prime}}^{\mathrm{red}} \rightarrow 1
$$

with $U$ unipotent and $\overline{\mathcal{H}}_{\Omega, \kappa^{\prime}}^{\text {red }}$ (split) reductive over $\kappa^{\prime}$. Now the maximal reductive quotient $M:=\overline{\mathcal{H}}_{\Omega}^{\text {red }}$ of $\overline{\mathcal{H}}_{\Omega}=\mathcal{H}_{\Omega} \otimes_{\tilde{\mathcal{O}}_{0}[v]} \tilde{\mathcal{O}}_{0}$ is a Chevalley (reductive) group scheme over $\tilde{\mathcal{O}}_{0}$. Since $\gamma_{0}(\Omega)=\Omega$ we have an action of $\gamma_{0}$ on $M$. Since $\gamma_{0}$ has order prime to $p$, we can see that $\mathrm{H}^{1}\left(\left\langle\gamma_{0}\right\rangle, U\right)=(0)$. Also, by filtering $U$ by vector groups we can see that $U^{\gamma_{0}}$ is connected (see [74, 4.7.2]). It follows that the group of connected components of $\mathcal{G}_{\Omega}^{\prime} \otimes_{\mathcal{O}[u]} \kappa^{\prime}$ is identified with that of $\left(\overline{\mathcal{H}}_{\Omega, \kappa^{\prime}}^{\text {red }}\right)^{\gamma_{0}}=\left(\overline{\mathcal{H}}_{\Omega}^{\text {red }}\right)^{\gamma_{0}} \otimes_{\mathcal{O}^{\prime}} \kappa^{\prime}=M^{\gamma_{0}} \otimes_{\tilde{\mathcal{O}}_{0}} \kappa^{\prime}$. We can now see that the $\gamma_{0}$-action on $M$ satisfies the assumptions of Proposition 1.4 i.e preserves a pair of maximal split torus and a Borel subgroup that contains it: Indeed, by construction, $\gamma_{0}$ preserves the maximal torus given by $T_{H}$. Now, as before, consider the subset $\Phi_{\Omega}$ of the set of roots $\Phi$ such that there is an affine root with vector part $a$ and defining a hyperplane containing $\Omega$. The set $\Phi_{\Omega}$ can be identified with the roots of $M$ with respect to the maximal torus given by $T_{H}$. The group $\left\langle\gamma_{0}\right\rangle$ acts on $\Phi_{\Omega}$. The intersection $\Phi_{\Omega}^{+}:=\Phi^{+} \cap \Phi_{\Omega}$ is a system of positive roots in $\Phi_{\Omega}$ which is stable under $\gamma_{0}$. Let $C$ be the affine chamber containing $\Omega$ in its closure which is given by $\Phi_{\Omega}^{+}$; this provides us with a $\gamma_{0}$-stable Borel subgroup in $M$ which contains $T_{H}$. We can now apply Proposition 1.4 to 
the Chevalley group $M=\overline{\mathcal{H}}_{\Omega}^{\text {red }}$ over $\tilde{\mathcal{O}}_{0}$ and the automorphism induced by $\gamma_{0}$ as above. We obtain that the group scheme of connected components of $\mathcal{G}_{\Omega}^{\prime} \otimes_{\mathcal{O}[u]} \kappa^{\prime}$ is given by the fibers of a finite étale commutative group scheme of order annihilated by $e$ and this order is the same for $\kappa=F$ or $\kappa=k$. Since $\mathcal{G}_{\Omega}^{\prime \prime} \otimes_{\mathcal{O}[u]} F$ is the neutral component of $\mathcal{G}_{\Omega}^{\prime} \otimes_{\mathcal{O}[u]} F$, the base change $\mathcal{G}_{\Omega}^{\prime \prime} \otimes_{\mathcal{O}[u]} \tilde{F}_{0}$ is also connected ([65, Exp. $\left.\mathrm{VI}_{\mathrm{A}}, 2.1 .1\right]$ ). The above now implies that $\mathcal{G}_{\Omega}^{\prime \prime} \otimes_{\mathcal{O}[u]} \tilde{k}$ and hence $\mathcal{G}_{\Omega}^{\prime \prime} \otimes_{\mathcal{O}[u]} k$ is also connected. Therefore, $\mathcal{G}_{\Omega}^{\prime \prime}=\left(\mathcal{G}_{\Omega}^{\prime}\right)^{0}$. We set $\mathcal{G}_{\Omega}:=\mathcal{G}_{\Omega}^{\prime \prime}=\left(\mathcal{G}_{\Omega}^{\prime}\right)^{0}$. It remains to show that the base changes of $\mathcal{G}_{\Omega}$ by $\mathcal{O}[u] \rightarrow \mathcal{O}$, $u \mapsto \varpi$, resp. $\mathcal{O}[u] \rightarrow \kappa[[u]]$, are isomorphic to the parahoric group schemes $\mathcal{P}_{x}$, resp. $\mathcal{P}_{x_{\kappa((u))}}$.

For simplicity, set $L=\bar{\kappa}((u)), L^{\prime}=\bar{\kappa}((v)), R=\bar{\kappa}[[u]], R^{\prime}=\bar{\kappa}[[v]]$ and denote by $H\left(L^{\prime}\right)_{\Omega} \subset H\left(L^{\prime}\right)$ the stabilizer of $\Omega \subset \mathcal{B}(H, L)=\mathcal{B}\left(H, L^{\prime}\right)^{\gamma_{0}}$. We also set $\mathcal{G}(L)=$ $\left(H\left(L^{\prime}\right)\right)^{\gamma_{0}}$ (the points of a connected reductive group over $L$ ). Notice that by construction, $\mathcal{G}_{\Omega}^{\prime}(R)=\mathcal{H}_{\Omega}\left(R^{\prime}\right)^{\gamma_{0}}$ while the result in the split case together with [7, 4.6] gives $\mathcal{H}_{\Omega}\left(R^{\prime}\right) \subset$ $H\left(L^{\prime}\right)_{\Omega}$ with finite index. Hence, we have

$$
\mathcal{G}_{\Omega}^{\prime}(R)=\mathcal{H}_{\Omega}\left(R^{\prime}\right)^{\gamma_{0}} \subset\left(H\left(L^{\prime}\right)_{\Omega}\right)^{\gamma_{0}}=\left(H\left(L^{\prime}\right)^{\gamma_{0}}\right)_{\Omega}=\mathcal{G}(L)_{\Omega}
$$

and we see that $\mathcal{G}_{\Omega}^{\prime}(R)$ is of finite index in the stabilizer $\mathcal{G}(L)_{\Omega}$ of $\Omega \subset \mathcal{B}(\mathcal{G}, L)$ in $\mathcal{G}(L)$. Since $\mathcal{G}_{\Omega}$ is the neutral component of $\mathcal{G}_{\Omega}^{\prime}$ we conclude that $\mathcal{G}_{\Omega} \otimes_{\mathcal{O}[u]} R$ is the smooth connected stabilizer of $\Omega$, i.e we have $\mathcal{G}_{\Omega} \otimes_{\mathcal{O}[u]} R=\mathcal{P}_{x_{\kappa((u))}} \otimes_{\kappa[[u]]} \bar{\kappa}[[u]]$. By [7, 1.7], this shows the desired result for the base change $\mathcal{O}[u] \rightarrow \kappa[[u]]$. The case $\mathcal{O}[u] \rightarrow \mathcal{O}, u \rightarrow \varpi$ is similar. This concludes the proof of Theorem 3.1 in the quasi-split case.

(c) Finally, we consider the general case. Recall our notations and in particular the choice of $\operatorname{Int}(\mathrm{g})$ in ${\underline{N^{\prime}}}^{*}\left(\breve{\mathcal{O}}\left[u^{ \pm 1}\right]\right)$. This gives the semilinear

$$
{ }^{*} \sigma:=\operatorname{Int}(\mathbf{g}) \cdot \sigma: \underline{G}^{*} \otimes_{\mathcal{O}} \breve{\mathcal{O}} \rightarrow \underline{G}^{*} \otimes_{\mathcal{O}} \breve{\mathcal{O}}
$$

which covers the Frobenius $\breve{\mathcal{O}}\left[u^{ \pm 1}\right] \rightarrow \breve{\mathcal{O}}\left[u^{ \pm 1}\right]$. We also have the inner twist $\underline{G}$ of $\underline{G}^{*}$ over $\mathcal{O}\left[u^{ \pm 1}\right]$ defined by taking ${ }^{*} \sigma$ fixed points of $\underline{G} \otimes_{\mathcal{O}} \breve{\mathcal{O}}$ as in (2.12). Our construction applied to the quasi-split $G_{\breve{F}}^{*}$ and the point $x^{*}$ given as $\psi_{*}(x)$ provides with a group scheme $\mathcal{G}_{x^{*}}^{*}$ over $\breve{\mathcal{O}}[u]$ which satisfies the conclusions of the Theorem. In particular, we have

$$
\left.\mathcal{G}_{x^{*}}^{*}\right|_{\breve{\mathcal{O}}\left[u^{ \pm 1}\right]}=\underline{G}^{*} \otimes_{\mathcal{O}} \breve{\mathcal{O}} .
$$

We will now show that ${ }^{*} \sigma: \underline{G}^{*} \otimes_{\mathcal{O}} \breve{\mathcal{O}} \rightarrow \underline{G}^{*} \otimes_{\mathcal{O}} \breve{\mathcal{O}}$ extends to an $\sigma$-semilinear

$$
{ }^{*} \sigma: \mathcal{G}_{x^{*}}^{*} \rightarrow \mathcal{G}_{x^{*}}^{*}
$$

We first verify that it is enough to check that the base change of * $\sigma$ over $\breve{F}((u))$ extends to $\breve{F}[[u]]$ : Indeed, since $\mathcal{G}_{x^{*}}^{*}=\operatorname{Spec}(A)$ is affine and smooth over $\breve{\mathcal{O}}[u]$ we can write $A=A\left[u^{-1}\right] \cap\left(A \otimes_{\mathcal{\mathcal { O }}[u]} \breve{F}[[u]]\right)$. Since ${ }^{*} \sigma$ is defined over $\mathcal{O}\left[u, u^{-1}\right]$, it remains to check that ${ }^{*} \sigma$ preserves $A \otimes_{\mathcal{\mathcal { O }}[u]} \breve{F}[[u]] \subset A \otimes_{\mathcal{\mathcal { O }}[u]} \breve{F}((u))$. Now let check that the base change of * $\sigma$ over $\breve{F}((u))$ extends to $\breve{F}[[u]]$ : Consider $x_{\breve{F}((u))}^{*}$ which by our construction is fixed by $\operatorname{Int}(\mathbf{g}) \cdot \sigma$. This implies that

$$
{ }^{*} \sigma\left(\mathcal{P}_{x_{\breve{F}((u))}^{*}}(\breve{F}[[u]])\right) \subset \mathcal{P}_{x_{\breve{F}((u))}^{*}}(\breve{F}[[u]]) .
$$


Since $F[[u]]$ is henselian and $F$ infinite and perfect, condition (ET 2) of [7, 1.7.2] is satisfied. Therefore, (3.5) implies that ${ }^{*} \sigma$ extends to $\mathcal{P}_{x_{F((u))}^{*}}$ which, by our construction, is the base change of the group scheme $\mathcal{G}_{x^{*}}^{*}$ to $\breve{F}[[u]]$.

We now define $\mathcal{G}_{x}$ to be the group scheme over $\mathcal{O}[u]$ given by the Weil descent datum provided by the action of ${ }^{*} \sigma=\operatorname{Int}(\mathbf{g}) \cdot \sigma$ on $\mathcal{G}_{x^{*}}^{*}$ over $\breve{\mathcal{O}}[u]$. Since $\mathcal{G}_{x^{*}}^{*}$ is affine, we can indeed see that $\mathcal{G}_{x}$ is represented by an affine group scheme over $\mathcal{O}[u]$, which then satisfies all the requirements in the Theorem.

3.c. In this section we start with a connected reductive group $G$ over the $p$-adic field $F$ that splits over the tamely ramified $\tilde{F} / F$ and $x \in \mathcal{B}(G, F)$ a point in the building of $G$. Let $\mathcal{P}_{x}$ be the corresponding parahoric group scheme over $\mathcal{O}$. We will apply Theorem 3.1 to construct a suitable group scheme $\mathcal{G}=\mathcal{G}_{x}$ over $\mathcal{O}[u]$ (see Corollary 3.2 below).

3.c.1. Starting from the above data, we choose a rigidification $(G, A, S, P)$ such that $x \in \mathcal{A}(G, A, F)$. Choose also a pinned split form $H$ of $G$ over $\mathcal{O}$ and let $(\underline{G}, \underline{A}, \underline{S}, \underline{P})$ be the corresponding rigidified group over $\mathcal{O}\left[u^{ \pm 1}\right]$ as constructed in 92. c.2. Let $\psi$ : $(\underline{G}, \underline{S}, \underline{P}) \otimes \breve{\mathcal{O}}\left[u^{ \pm 1}\right] \stackrel{\sim}{\rightarrow}\left(\underline{G}^{*}, \underline{S}^{*}, \underline{P}^{*}\right) \otimes \breve{\mathcal{O}}\left[u^{ \pm 1}\right]$ be an inner twist and $\underline{c}^{\text {rig }}$ be the corresponding cocycle. Let $\left(G_{0}, A_{0}, S_{0}, P_{0}\right):=\left(\underline{G}_{F}, \underline{A}_{F}, \underline{S}_{F}, \underline{P}_{F}\right)$ be the base change via $\mathcal{O}\left[u^{ \pm 1}\right] \rightarrow F$ given by $u \mapsto \varpi$. The specialization of the inner twist by $u \mapsto \varpi$ is denoted by $\psi_{0}$. The set of inner twists

$$
\psi:(G, S, P) \otimes_{F} \breve{F} \stackrel{\sim}{\rightarrow}\left(G^{*}, S^{*}, P^{*}\right) \otimes_{F} \breve{F}
$$

such that $\psi \cdot \sigma(\psi)^{-1}=\left.\underline{c}^{\text {rig }}(1)\right|_{u=\varpi}$ forms an $N^{\prime}(F)$-torsor, where $N^{\prime}=N_{M^{\prime}}(T)$. As the cocycles corresponding to $\psi$ and $\psi_{0}$ are the same, the morphism $\psi_{0} \psi^{-1}$ is defined over $F$. Therefore, the choice of a rigidification $(G, A, S, P)$ of $G$ produces an isomorphism $\alpha: G \stackrel{\sim}{\rightarrow} G_{0}=\underline{G}_{F}$, which is well-defined up to the action of $N^{\prime}(F)$. Since $N^{\prime}$ centralizes $A, N^{\prime}(F)$ acts trivially on $\mathcal{A}(G, A, F)$; hence, $x$ corresponds to a well-defined point $x_{0}$ in $\mathcal{A}\left(\underline{G}_{F}, \underline{A}_{F}, F\right)$. Note that, however, $x_{0}$ depends on the choice of the rigidification $(G, A, S, P)$.

Let $\left(G, A^{\prime}, S^{\prime}, P^{\prime}\right)$ be another choice of rigidification of $G$ and let $x_{0}^{\prime} \in \mathcal{A}\left(\underline{G}_{F}, \underline{A}_{F}, F\right)$ be the corresponding point. By Lemma 1.8, there is $g \in G_{\text {ad }}(F)$ sending $(A, S, P)$ to $\left(A^{\prime}, S^{\prime}, P^{\prime}\right)$. Therefore, as points in the building of $G_{0}=\underline{G}_{F}, x_{0}$ and $x_{0}^{\prime}$ are in the same $G_{0, \text { ad }}(F)$-orbit. Therefore, there is an element $n$ in $N_{G_{0, \text { ad }}}\left(A_{0}\right)$ that sends $x_{0}$ to $x_{0}^{\prime}$.

Now apply Theorem 3.1 to $(\underline{G}, \underline{A}, \underline{S}, \underline{P})$ and $x_{0} \in \mathcal{A}\left(\underline{G}_{F}, \underline{A}_{F}, F\right)$. We obtain a group scheme $\mathcal{G}_{x_{0}}$ whose specialization along $u=\varpi$ gives back $\mathcal{P}_{x}$. Observe that $x_{0}$ is not uniquely determined by $x$, but also depends on the rigidification of $G$. However, as explained before, different $x_{0}$ 's are in the same $N_{G_{0, \text { ad }}}\left(A_{0}\right)(F)$-orbit. It is easy to see that an $N_{G_{0, \text { ad }}}\left(A_{0}\right)(F)$-orbit on $\mathcal{A}\left(G_{0}, A_{0}, F\right)$ is the same as an $\operatorname{Im}\left(N_{\underline{G}_{\text {ad }}}(\underline{A})\left(\mathcal{O}\left[u^{ \pm 1}\right]\right) \stackrel{u=\varpi}{\rightarrow}\right.$ $N_{G_{0, \text { ad }}}\left(A_{0}\right)(F)$ )-orbit. Therefore, different $\mathcal{G}_{x_{0}}$ 's are isomorphic to each other via conjugation by an element in $N_{\underline{G}_{\text {ad }}}(\underline{A})\left(\mathcal{O}\left[u^{ \pm 1}\right]\right)$ and so the isomorphism class of this group scheme is independent of choices; we will denote the group scheme by $\mathcal{G}_{x}$. In particular, we obtain:

Corollary 3.2. Starting with $G$ and $x$ as in the beginning of $3 . c$, there exists a smooth, affine group scheme $\mathcal{G}=\mathcal{G}_{x} \rightarrow \mathbb{A}_{\mathcal{O}}^{1}=\operatorname{Spec}(\mathcal{O}[u])$ with connected fibers such that 
1) The group scheme $\mathcal{G}_{\mid \mathcal{O}\left[u, u^{-1}\right]}$ is $\underline{G}$;

2) The base change of $\mathcal{G}$ under $\operatorname{Spec}(\mathcal{O}) \rightarrow \mathbb{A}_{\mathcal{O}}^{1}$ given by $u \mapsto \varpi$ is $\mathcal{P}_{x}$ and the base change of $\mathcal{G}$ under $\operatorname{Spec}(\kappa[[u]]) \rightarrow \mathbb{A}_{\mathcal{O}}^{1}$ given by $\mathcal{O}[u] \rightarrow \kappa[[u]]$ is $\mathcal{P}_{x_{\kappa((u))}}$.

\section{Classical groups}

Recall that when $G$ is a classical group over the local field $F$, Bruhat and Tits have given a description of the building $\mathcal{B}(G, F)$ as a set of certain norms on the space of the "natural representation" ([8], [10]). At least when $p$ is odd, this produces a description of the facets of the building in terms of self-dual lattice chains in this space. The corresponding parahoric group scheme can also be explicitly described as the neutral component of the polarized automorphisms of the lattice chain. In this chapter, we extend some of this picture to the group schemes over $\mathcal{O}[u]$ constructed in Theorem 3.1 .

4.a. Lattice chains. First we recall the set-up of lattice chains over $\mathcal{O}$ (cf. [8], [10], [55]).

4.a.1. Suppose first that $D$ is a central division $F$-algebra of degree $d$ and Brauer invariant $s / d$ with $0<s<d$ and $\operatorname{gcd}(s, d)=1$. Recall $\mathcal{O}$ has residue field $\mathbb{F}_{q}, q=p^{m}$. Let $F_{d}=F \mathbb{Q}_{p^{m d}}$ which is then an unramified extension of $F$ of degree $d$ with integers $\mathcal{O}_{d}$. Set $\sigma=$ Frob $_{p^{m}}$ for the generator of the Galois group of $F_{d} / F$. We can write

$$
D=F_{d} \oplus F_{d} \cdot \Pi \oplus \cdots \oplus F_{d} \cdot \Pi^{d-1}
$$

with relations $\Pi^{d}=\varpi, a \cdot \Pi=\Pi \cdot \sigma^{s}(a)$ for all $a \in F_{d}$. This contains the maximal order $\mathcal{O}_{D}=\mathcal{O}_{F_{d}} \oplus \mathcal{O}_{F_{d}} \cdot \Pi \oplus \cdots \oplus \mathcal{O}_{F_{d}} \cdot \Pi^{d-1}$.

Consider $V=D^{n}$ as a left $D$-module and let $G=\operatorname{GL}_{n}(D)=\operatorname{Aut}_{D}(V)$ identified by sending the matrix $A \in \mathrm{GL}_{n}(D)$ to the automorphism $x \mapsto x \cdot A^{-1}$. A lattice $\mathcal{L}$ in $V=D^{n}$ is a finitely generated $\mathcal{O}_{D}$-submodule of $V$ that contains a $D$-basis of $V$; then $\mathcal{L}$ is $\mathcal{O}_{D}$-free of rank $n$. Recall that a lattice chain in $V$ is a totally ordered (non-empty) set $\mathcal{L} \bullet$ of $\mathcal{O}_{D}$-lattices in $V$ which is stable under homotheties. It can be represented as:

$$
\cdots \subset \Pi \mathcal{L}_{0} \subset \mathcal{L}_{r-1} \subset \cdots \subset \mathcal{L}_{1} \subset \mathcal{L}_{0} \subset \cdots
$$

By [8], the facets $\Omega$ in $\mathcal{B}(G, F)$ correspond bijectively to $\mathcal{O}_{D^{-}}$-lattice chains (cf. [55]) in $V=D^{n}$ (Bruhat and Tits consider right modules but this is equivalent). Then the parahoric group scheme $\mathcal{P}_{x}(x \in \Omega)$ is the group scheme over $\mathcal{O}$ given by the $\mathcal{O}_{D}$-linear automorphisms of the corresponding chain, i.e

$$
\mathcal{P}_{x}(R)=\operatorname{Aut}_{\mathcal{O}_{D} \otimes_{\mathcal{O}} R}\left(\left\{\mathcal{L} \bullet \otimes_{\mathcal{O}} R\right\}\right)
$$


4.a.2. More generally suppose that $D$ is a central simple $L$-algebra with an involution $\tau$ such that $\tau\left(\mathcal{O}_{D}\right)=\mathcal{O}_{D}$; assume that $F$ is the fixed field of the involution on $L$. Let $\epsilon= \pm 1$ and let $h$ be an $\epsilon$-hermitian $D$-valued form on $V=D^{n}$ with respect to $\tau$. If $\mathcal{L}$ is an $\mathcal{O}_{D}$-lattice in $V$, we can consider its dual $\mathcal{L}^{\vee}=\left\{x \in \mathcal{L} \mid h(x, \lambda) \in \mathcal{O}_{D}, \forall \lambda \in \mathcal{L}\right\}$. A lattice chain $\mathcal{L}_{\bullet}$ is called self dual if $\mathcal{L} \in \mathcal{L}_{\bullet}$ if and only if $\mathcal{L}^{\vee} \in \mathcal{L}_{\bullet}$. The form $h$ defines an involution $*$ on $\mathrm{GL}_{n}(D)$ by $h(x A, y)=h\left(x, y A^{*}\right)$. Consider the unitary group $\mathrm{U}(V, h)=\left\{A \in \mathrm{GL}_{n}(D) \mid\left(A^{*}\right)^{-1}=A\right\}$ given by elements of $\mathrm{GL}_{n}(D)$ that respect $h$ and let $G$ be its neutral component. Recall that we assume $p$ is odd. By [53], the building $\mathcal{B}(G, F)$ can be identified with the fixed points of the action induced by $A \mapsto\left(A^{*}\right)^{-1}$ on $\mathcal{B}\left(\mathrm{GL}_{n}(D), L\right)$. Using the above, we now see that facets $\Omega$ in $\mathcal{B}(G, F)$ correspond to self-dual $\mathcal{O}_{D}$-lattice chains $\mathcal{L}_{\bullet}$ in $V$. (This also follows from the explicit description in [10], noting that when $p \neq 2$, the maximinorante norms of loc. cit. can be described via self-dual graded lattice chains). It then also follows that the parahoric Bruhat-Tits $\mathcal{P}_{x}$ ( $x$ a generic point in $\Omega$ ) is the neutral component of the group scheme over $\mathcal{O}$ given by $\mathcal{O}_{D}$-linear automorphisms of the chain $\mathcal{L} \bullet$ that respect the perfect forms $\mathcal{L}_{i} \times \mathcal{L}_{j} \rightarrow \mathcal{O}_{D}$ obtained from $h$. There is also a similar description for unitary similitude groups.

We now extend most of this picture to the group schemes over $\mathcal{O}[u]$. We first start by describing some cases of split groups.

\section{4.b. Some split classical groups.}

4.b.1. The case of $\mathrm{GL}_{N}$. Suppose $W=\mathcal{O}[u]^{N}$ and set $\bar{W}=W \otimes_{\mathcal{O}[u], u \mapsto 0} \mathcal{O}$. Write $\bar{W}=\oplus_{j=0}^{r-1} V_{i}$ and consider the parabolic subgroup $Q \subset \mathrm{GL}(\bar{W})$ which is the stabilizer of the flag $F_{i}=\oplus_{j \geq i} V_{j}$ given by the $V_{i}$. Denote by $d_{i}$ the $\mathcal{O}$-rank of $V_{i}$. For $0 \leq i \leq r-1$, set $W_{i}$ for the preimage of $F_{i}$ under $W \rightarrow \bar{W}$ so that

$$
u W \subset W_{r-1} \subset \cdots \subset W_{1} \subset W_{0}=W .
$$

Extend the index set by requiring $W_{i+k \cdot r}=u^{k} W_{i}$ for $k \in \mathbb{Z}$. Denote by $\iota_{i}: W_{i+1} \rightarrow W_{i}$ the inclusion. We have a natural identification $\mathrm{GL}\left(W_{i}\right)=\mathrm{GL}\left(W_{i+r}\right)$ given by conjugating by $u$.

The dilatation $\mathcal{G}=\mathrm{GL}(W)_{Q}$ of $\mathrm{GL}(W)$ along $Q$ is isomorphic to the closed subgroup scheme in $H=\prod_{i=0}^{r-1} \mathrm{GL}\left(W_{i}\right)=\prod_{i \in \mathbb{Z} / r \mathbb{Z}} \mathrm{GL}\left(W_{i}\right)$ of tuples that commute with the maps $\iota_{i}: W_{i} \rightarrow W_{i+1}$. It is isomorphic to the group scheme over $\mathcal{O}[u]$ obtained by Corollary 3.2 applied to $G=\mathrm{GL}_{N}$ and a point $x$ corresponding to the lattice chain $\left\{W_{i} \otimes_{\mathcal{O}[u], u \mapsto \varpi} \mathcal{O}\right\}_{i}$. (The lattice chains $\left\{W_{i} \otimes_{\mathcal{O}[u]} \kappa((u))\right\}_{i}$ correspond to $x_{\kappa((u))}$.)

4.b.2. The case of $\mathrm{GSp}_{2 n}$. Consider $W=\oplus_{i=1}^{2 n} \mathcal{O}[u] \cdot e_{i}$ with the perfect $\mathcal{O}[u]$-bilinear alternating form $h: W \times W \rightarrow \mathcal{O}[u]$ determined by $h\left(e_{i}, e_{2 n+1-j}\right)=\delta_{i j}, h\left(e_{i}, e_{j}\right)=$ $h\left(e_{2 n+1-i}, e_{2 n+1-j}\right)=0$ for $1 \leq i, j \leq n$. Let us fix a chain of $\mathcal{O}[u]$-submodules

$$
u W \subset W_{r-1} \subset \cdots \subset W_{1} \subset W_{0} \subset W
$$

such that

(i) $W_{i}^{\vee}=u^{-1} W_{r-i-1}$, for $0 \leq i \leq r-1$,

(ii) $W_{i} / W_{i+1} \simeq \mathcal{O}^{d_{i}}$. 
Again, extend the index set by periodicity by setting $W_{i+k \cdot r}=u^{k} W_{i}$ so that the form $h$ gives $W_{i}^{\vee}=W_{-i-a}$, with $a=0$ or 1 , and set $\iota_{i}: W_{i+1} \rightarrow W_{i}$ as before. Consider the group scheme $\mathcal{G}$ over $\mathcal{O}[u]$ of similitude automorphisms of the "polarized" system $\left(W_{i}, h_{i}\right)_{i \in \mathbb{Z}}$; more precisely this is the subgroup scheme of $\mathbb{G}_{\mathrm{m}} \times \prod_{i=0}^{r-1} \mathrm{GL}\left(W_{i}\right)=\mathbb{G}_{\mathrm{m}} \times \prod_{i \in \mathbb{Z} / r \mathbb{Z}} \mathrm{GL}\left(W_{i}\right)$ consisting of $\left(c,\left(g_{i}\right)\right)$ such that

$$
h\left(g_{i}(x), g_{-i-a}(y)\right)=c \cdot h(x, y), \quad \text { for all } i \in \mathbb{Z} .
$$

As in [55, Appendix to Ch. 3], we can see that $\mathcal{G}$ is smooth over $\mathcal{O}[u]$; it is isomorphic to the group scheme obtained by Corollary 3.2 applied to $G=\mathrm{GSp}_{2 n}$ and a point $x$ corresponding to the self-dual lattice chain $\left\{W_{i} \otimes_{\mathcal{O}[u], u \mapsto \varpi} \mathcal{O}\right\}_{i}$.

4.c. Non-split classical groups. We now extend this to (essentially) the general classical case. When in the sections below we consider symmetric or hermitian forms, we will assume that the prime $p$ is odd. We first mostly concentrate on describing explicitly the group schemes $\underline{G}$.

4.c.1. Division algebras. With the notations of 4.a.1, consider the associative (central) $\mathcal{O}[u]$-algebra given by

$$
\mathcal{O}(\mathcal{D})=\mathcal{O}_{d}[u] \oplus \mathcal{O}_{d}[u] \cdot X \oplus \cdots \oplus \mathcal{O}_{d}[u] \cdot X^{d-1},
$$

with relations $X^{d}=u, f \cdot X=X \cdot \sigma(f)^{s}$ for $f \in \mathcal{O}_{d}[u]$ with $\sigma\left(\sum a_{i} v^{i}\right)=\sum \sigma\left(a_{i}\right) v^{i}$. Notice that $\mathcal{D}:=\mathcal{O}(\mathcal{D})\left[u^{-1}\right]$ is an Azumaya algebra over $\mathcal{O}\left[u^{ \pm 1}\right]$ which splits after the unramified extension $\mathcal{O}\left[u^{ \pm 1}\right] \rightarrow \mathcal{O}_{d}\left[u^{ \pm 1}\right]$; then $\mathcal{O}(\mathcal{D})$ is a maximal order in this Azumaya algebra. We have isomorphisms

$$
\mathcal{D} \otimes_{\mathcal{O}\left[u^{ \pm 1}\right]} F \simeq D, \quad \mathcal{O}(\mathcal{D}) \otimes_{\mathcal{O}[u]} \mathcal{O}_{F} \simeq \mathcal{O}_{D}
$$

where the ring homomorphisms are given by $u \mapsto \varpi$. In addition, reducing $\mathcal{O}(\mathcal{D})$ modulo $\varpi$ followed by completing at $(u)$ also produces a maximal order in a central division algebra of degree $d$ and invariant $s / d$ over the local field $\mathbb{F}_{p^{m}}((u))$.

For $n \geq 1$, we can consider the affine group scheme over $\mathcal{O}\left[u^{ \pm 1}\right]$ given by

$$
R \mapsto \operatorname{Aut}_{\mathcal{D} \otimes_{\mathcal{O}[u \pm 1} R}\left(\mathcal{D}^{n} \otimes_{\mathcal{O}\left[u^{ \pm 1}\right]} R\right) .
$$

We can see directly from the construction of $\$ 2 . c$ that this group scheme is isomorphic to $\underline{G}$ for $G=\mathrm{GL}_{n}(D)$.

We can also consider the affine group scheme $\mathcal{G}$ over $\mathcal{O}[u]$ given by

$$
\mathcal{G}(R):=\operatorname{Aut}_{\mathcal{O}(\mathcal{D}) \otimes_{\mathcal{O}[u]}}\left(\mathcal{O}(\mathcal{D})^{n} \otimes_{\mathcal{O}[u]} R\right) .
$$

The group $\mathcal{G}$ is smooth over $\mathcal{O}[u]$ and is isomorphic to the group scheme of Corollary 3.2 for the vertex corresponding to the lattice chain given by the multiples of $\mathcal{O}_{D}$.

4.c.2. In what follows, the base is $\mathbb{Q}_{p}$ and we will be discussing group schemes over $\mathbb{Z}_{p}\left[u^{ \pm 1}\right]$. We assume $F$ is a tame finite extension of $\mathbb{Q}_{p}$ and let $\mathbb{Q}_{p^{r}}=\operatorname{Frac}\left(W\left(\mathbb{F}_{p^{r}}\right)\right)$ the maximal unramified extension of $\mathbb{Q}_{p}$ contained in $F$. Denote by $\mathbb{Z}_{p^{r}}$ the ring of integers $W\left(\mathbb{F}_{p^{r}}\right)$ of $\mathbb{Q}_{p^{r}}$. When $r$ is clear, we will simply write $W$ for $\mathbb{Z}_{p^{r}}=W\left(\mathbb{F}_{p^{r}}\right)$. We will then denote by $W_{d}$ the integers of the unique unramified extension $W\left(\mathbb{F}_{p^{r d}}\right)$ of $W$ of degree $d \geq 2$. 
4.c.3. We can now explicitly construct the group scheme $\underline{G}$ over $\mathbb{Z}_{p}\left[u^{ \pm 1}\right]$ associated to the restriction of scalars $G=\operatorname{Res}_{F / \mathbb{Q}} \mathrm{GL}_{m}(D)$ with $D$ a division algebra over $F$ as above. We choose a $W$-algebra isomorphism $j: W[x] /\left(x^{e}-p c\right) \stackrel{\sim}{\rightarrow} \mathcal{O}_{F}$ where $p \nmid e$ and $c \in W^{*}$; i.e a uniformizer $\varpi$ of $F$ such that $\varpi^{e}$ is in $W$.

As above, we construct an associative (central) $W[v]$-algebra given by

$$
\mathcal{O}(\mathcal{D})=W_{d}[v] \oplus W_{d}[v] \cdot X \oplus \cdots \oplus W_{d}[v] \cdot X^{d-1},
$$

with relations $X^{d}=v, f \cdot X=X \cdot \sigma(f)^{s}$ for $f \in W_{d}[v]$ with $\sigma\left(\sum a_{i} v^{i}\right)=\sum \sigma\left(a_{i}\right) v^{i}$. (After the base change $W[v] \rightarrow \mathcal{O}[u], v=u$, this produces the algebra denoted by the same symbol in the previous paragraph.) Again, $\mathcal{D}=\mathcal{O}(\mathcal{D})\left[v^{-1}\right]$ is an Azumaya algebra over $W\left[v^{ \pm 1}\right]$. We have isomorphisms

$$
\mathcal{D} \otimes_{W\left[v^{ \pm 1}\right]} F \simeq D, \quad \mathcal{O}(\mathcal{D}) \otimes_{W[v]} \mathcal{O}_{F} \simeq \mathcal{O}_{D}
$$

where $W\left[v^{ \pm 1}\right] \rightarrow F, W[v] \rightarrow \mathcal{O}_{F}$, are given by $v \mapsto \varpi$. In addition, reducing $\mathcal{O}(\mathcal{D})$ modulo $p$ followed by completing at $(v)$ also produces a maximal order in a central division algebra of degree $d$ and invariant $s / d$ over the local field $\mathbb{F}_{p^{r}}((v))$.

Define $\phi: \mathbb{Z}_{p}[u] \rightarrow \mathbb{Z}_{p^{r}}[v]$ by $u \mapsto v^{e} \cdot c^{-1}$ with $c=\varpi^{e} \cdot p^{-1}$. For $m \geq 1$, we set $M=\mathcal{O}(\mathcal{D})^{m}$. We consider

$$
\mathcal{G}^{\prime}(R)=\operatorname{Aut}_{\mathcal{O}(\mathcal{D}) \otimes_{\mathbb{Z}_{p}[u]} R}\left(M \otimes_{\mathbb{Z}_{p}[u]} R\right) .
$$

This defines a smooth affine group scheme over $\mathbb{Z}_{p}[u]$ such that

$$
\mathcal{G}^{\prime} \otimes_{\mathbb{Z}_{p}[u], u \mapsto p} \mathbb{Q}_{p} \simeq \operatorname{Res}_{F / \mathbb{Q}_{p}}\left(\operatorname{GL}_{m}(D)\right) .
$$

Suppose we choose another uniformizer $\varpi_{1}$ with $\varpi_{1}^{e}=p c_{1}$ and denote by $\phi_{1}$ the corresponding map as above. Let $y=\varpi_{1} / \varpi \in \mathcal{O}_{F}^{*}$. Since $y^{e} \in W^{*}$ and $p \nmid e$, the extension $\mathbb{Q}_{p^{r}}(y) / \mathbb{Q}_{p^{r}}$ is unramified and therefore $y$ is in $W^{*}=\mathbb{Z}_{p^{r}}^{*}$. Sending $v$ to $y \cdot v$ then gives an isomorphism $\alpha: W[v] \stackrel{\sim}{\rightarrow} W[v]$ that maps $\left(v^{e}-p c\right)$ to $\left(v^{e}-p c_{1}\right)$ and commutes with $\phi, \phi_{1}$. Find $z \in W_{d}^{*}$ such that $N_{\mathbb{Q}_{p^{r d}} / \mathbb{Q}_{p} r}(z)=y$; sending $X \mapsto X \cdot z$ gives $\mathcal{O}(\mathcal{D}) \stackrel{\sim}{\rightarrow} \mathcal{O}(\mathcal{D}) \otimes_{W[v], \alpha} W[v]$. This implies that $\mathcal{G}^{\prime}$ is independent from the choice of $\varpi$ with $\varpi^{e} \in W$. The group scheme $\mathcal{G}_{\mid \mathbb{Z}_{p}\left[u^{ \pm 1}\right]}^{\prime}$ is isomorphic to the group scheme $\underline{G}$ obtained from $G=\operatorname{Res}_{F / \mathbb{Q}_{p}}\left(\mathrm{GL}_{m}(D)\right)$ as above; this follows directly from the construction of $92 . \mathrm{C}$ using (2.2) . The restriction $\mathcal{G}_{\mid \mathbb{Z}_{p}\left[u^{ \pm 1}\right]}^{\prime} \rightarrow \operatorname{Spec}\left(\mathbb{Z}_{p}\left[u^{ \pm 1}\right]\right)$ is the Weil restriction of scalars from $W\left[v^{ \pm 1}\right]$ of a twisted form of $\mathrm{GL}_{m d}$ over $W\left[v^{ \pm 1}\right]$; this twisted form is the group of automorphisms of the module $\mathcal{D}^{m}=\mathcal{O}(\mathcal{D})\left[v^{-1}\right]^{m}$ for the Azumaya algebra $\mathcal{D}$ over $W\left[v^{ \pm 1}\right]$.

4.c.4. Here again $W$ is $\mathbb{Z}_{p^{r}}=W\left(\mathbb{F}_{p^{r}}\right)$ and $W_{d}=W\left(\mathbb{F}_{p^{r d}}\right)$ as above. Write

$$
W\left[v^{ \pm 1}\right]^{*} /\left(W\left[v^{ \pm 1}\right]^{*}\right)^{2}=\{1, \alpha, v, \alpha v\}
$$

where $\alpha$ is an element of $W^{*}$ which is not a square.

We consider a $W[v]$-algebra $\mathfrak{R}$ given as

$$
\mathfrak{R}=W_{2}[v], \quad \text { or } \quad \mathfrak{R}=W\left[v^{\prime}\right], v \mapsto v^{\prime 2}, \quad \text { or } \quad \mathfrak{R}=W\left[v^{\prime}\right], v \mapsto \alpha^{-1} v^{\prime 2} .
$$


We will refer to the first possibility as the unramified case. The other two possibilities are the ramified case. We have a $W[v]$-symmetric bilinear form

$$
h_{\mathfrak{R}}: \mathfrak{R} \times \mathfrak{R} \rightarrow W[v] ; \quad h_{\mathfrak{R}}(x, y)=\frac{1}{2} \operatorname{Tr}_{\mathfrak{R} / W[v]}(x \bar{y})
$$

where $r \mapsto \bar{r}$ is the order two automorphism of $\mathfrak{R}$ over $W[v]$. The form $h_{\Re}$ is perfect in the unramified case; $h_{\mathfrak{R}}\left[v^{-1}\right]$ on $\mathfrak{R}\left[v^{-1}\right]$ is always perfect.

We also consider the central $W[v]$-algebra

$$
\mathcal{O}(\mathcal{Q})=W_{2}[v] \oplus W_{2}[v] \cdot X
$$

with relations $X^{2}=v, f \cdot X=X \cdot \sigma(f)$ for $f \in W_{2}[v]$; this corresponds to the quaternion case $(s, d)=(1,2)$ as above. Denote by $x \mapsto \bar{x}$ the main involution of $\mathcal{O}(\mathcal{Q})$ which is $\sigma$ on $W_{2}[v]$ and maps $X$ to $-X$. Let $\zeta$ be a root of unity that generates $W_{2}$ over $W$, $W_{2}=W(\zeta)$. Then $\bar{\zeta}=-\zeta$. The reduced norm $\operatorname{Norm}(r)=r \cdot \bar{r}$ defines a $W[v]$-linear quadratic form on $\mathcal{O}(\mathcal{Q})$. Denote by $h_{\mathcal{O}(\mathcal{Q})}: \mathcal{O}(\mathcal{Q}) \times \mathcal{O}(\mathcal{Q}) \rightarrow W[v]$ the corresponding $W[v]$-bilinear symmetric form.

We will use the symbol $\mathfrak{O}$ to denote one of $W[v], \mathfrak{R}$, or $\mathcal{O}(\mathcal{Q})$; each of these $W[v]$ algebras supports an involution $x \mapsto \bar{x}$ as above (this is trivial in the case of $W[v]$ ). In the following paragraph we will give each time a free (left) module $M$ over $\mathfrak{O}$ which is equipped with a certain form $h$ (alternating, symmetric, hermitian, etc.). All the forms below are "perfect" after we invert $v$, i.e over $W\left[v^{ \pm 1}\right]$. We will consider the group scheme $\mathcal{G}^{\prime}$ over $W[v]$ given by the $\mathfrak{O}$-module automorphisms of $M$ that respect the corresponding form $h$. Suppose that $F$ is a totally tamely ramified extension of $\mathbb{Q}_{p^{r}}$ of degree e. Choose a uniformizer $\varpi$ of $F$ with $\varpi^{e} \in W$ as above and consider the base change $W\left[v^{ \pm 1}\right] \rightarrow F$ given by $v \rightarrow \varpi$. In the list below, we mention the type of the isogeny class of the group $\left.\mathcal{G}^{\prime}\right|_{W\left[v^{ \pm 1}\right]} \otimes_{W\left[v^{ \pm 1}\right]} F$ over $F$ according to the tables 4.2 and 4.3 of [71, p. 60-65]. The determination of these types follows [71, 4.4, 4.5]. The corresponding symbol is read from the first column of these tables.

4.c.5. Alternating forms.

- $M=W[v]^{2 n}=\oplus_{i=1}^{2 n} W[v] \cdot e_{i}$ with the alternating $W[v]$-bilinear form $h$ determined by $h\left(e_{i}, e_{2 n+1-j}\right)=\delta_{i j}, 1 \leq i \leq n$. (cf. 4 .b.2). (For $n \geq 2$, the type is $C_{n}$.)

4.c.6. Symmetric forms. (Set $n=2 m+1$, or $n=2 m$.)

- Split: $M=W[v]^{n}=\oplus_{i=1}^{n} W[v] \cdot e_{i}$ with the symmetric $W[v]$-bilinear form $h=$ $h(n)$ determined by $h(n)\left(e_{i}, e_{n+1-j}\right)=\delta_{i j}$. (For $n \geq 6$, the type is $B_{m}$, or $D_{m}$ respectively.)

- Quasi-split, even case: Here $n$ is even and $M=W[v]^{n-2} \oplus \Re$ with the symmetric $W[v]$-bilinear form $h$ given as the direct sum $h(n-2)+h_{\Re}$. (The types are ${ }^{2} D_{m}$ if $\mathfrak{R}=W_{2}[v]$ (unramified) and $n \geq 8$ and $C-B_{m-1}$ if $\mathfrak{R}$ is ramified and $n \geq 6$.)

- Non quasi-split, even case: Here $n$ is even and $M=W[v]^{n-4} \oplus \mathcal{O}(\mathcal{Q})$ with the symmetric $W[v]$-bilinear form $h$ given as the direct sum $h(n-4)+h_{\mathcal{O}(\mathcal{Q})}$. (For $n \geq 6$, the type is ${ }^{2} D_{m}^{\prime}$.) 
- Non quasi-split, odd case: Here $n$ is odd and $M=W[v]^{n-3} \oplus \mathcal{O}(\mathcal{Q})^{0}$ with the symmetric $W[v]$-bilinear form $h$ given as the direct sum $h(n-3)+h_{\mathcal{O}(\mathcal{Q})^{0}}$. We denote by $\mathcal{O}(\mathcal{Q})^{0}$ the submodule of elements $r$ for which $r+\bar{r}=0$ and by $h_{\mathcal{O}(\mathcal{Q})^{0}}$ the restriction of $h_{\mathcal{O}(\mathcal{Q})}$ to this submodule. (For $n \geq 6$, the type is ${ }^{2} B_{m}^{\prime}$.)

4.c.7. Hermitian forms. (Set $n=2 m+1$, or $n=2 m$.)

- quasi-split: $M=\mathfrak{R}^{n}$ with hermitian form $h=H(n)$ given by

$$
H(n)(x, y)=x^{t} \cdot K_{n} \cdot \bar{y}
$$

where $K_{n}$ is the antidiagonal $n \times n$ unit matrix. There are subcases here according to the choice of $\mathfrak{R}$. (Suppose $n \geq 3$. In the unramified case, the type is ${ }^{2} A_{n-1}^{\prime}$. In the ramified case, the type is $C-B C_{m}$ if $n=2 m+1$, or $B-C_{m}$ if $n=2 m$.)

- non quasi-split, ramified, even case: Here $n=2 m$ is even, $M=\mathfrak{R}^{n-2} \oplus \mathfrak{R}^{2}, \mathfrak{R}$ ramified with hermitian form $H$ given as the direct sum $h=H(n-2) \oplus H_{\alpha}$ with

$$
H_{\alpha}\left(\left(x_{1}, x_{2}\right),\left(y_{1}, y_{2}\right)\right)=x_{1} \bar{y}_{1}-\alpha \cdot x_{2} \bar{y}_{2}
$$

and $\alpha \in W^{*}$ which is not in $\left(W^{*}\right)^{2}$. (If $n \geq 3$, the type is ${ }^{2} B-C_{m}$.)

- non quasi-split, unramified, even case: Here again $n=2 m$ is even, $M=\mathfrak{R}^{n-2} \oplus$ $\mathfrak{R}^{2}, \mathfrak{R}=W_{2}[v]$, with hermitian form $h$ given as the direct sum $h=H(n-2) \oplus H_{u}$ with

$$
H_{u}\left(\left(x_{1}, x_{2}\right),\left(y_{1}, y_{2}\right)\right)=x_{1} \bar{y}_{1}-v \cdot x_{2} \bar{y}_{2} .
$$

(If $n \geq 3$, the type is ${ }^{2} A_{n-1}^{\prime \prime}$.)

4.c.8. Quaternionic $\epsilon$-hermitian forms. Let $\epsilon= \pm 1$. If $M$ is a left $\mathcal{O}(\mathcal{Q})$-module, then a $W[u]$-bilinear $H: M \times M \rightarrow \mathcal{O}(\mathcal{Q})$ is called an $\epsilon$-hermitian (i.e hermitian if $\epsilon=1$, anti-hermitian if $\epsilon=-1$ ) form, for the main involution $d \mapsto \bar{d}$, if it satisfies: $H(d x, y)=$ $d H(x, y), \overline{H(x, y)}=\epsilon H(y, x)$ for $d \in \mathcal{O}(\mathcal{Q}), x, y \in M$. Choose a unit $\xi \in W_{2}^{*}$ such that $\operatorname{Norm}(\xi)=-\operatorname{Norm}(\zeta)=\zeta^{2}$.

- Quaternionic hermitian: $M=\mathcal{O}(\mathcal{Q})^{n}$, with hermitian form $h=H(n): M \times M \rightarrow$ $\mathcal{O}(\mathcal{Q})$ given by

$$
H(n)(x, y)=x^{t} \cdot K_{n} \cdot \bar{y} .
$$

(If $n \geq 2$, the type is ${ }^{2} C_{n}$.)

- Quaternionic anti-hermitian: $M=\mathcal{O}(\mathcal{Q})^{m} \oplus \mathcal{O}(\mathcal{Q})^{m} \oplus M_{0}$ where $M_{0}=\mathcal{O}(\mathcal{Q})^{r}$, $n=2 m+r$. The anti-hermitian form $h={ }^{\prime} H: M \times M \rightarrow \mathcal{O}(\mathcal{Q})$ is the direct sum ${ }^{\prime} H(2 m) \oplus^{\prime} H_{0}$ where

$$
{ }^{\prime} H(2 m)(x, y)=x^{t} \cdot\left(\begin{array}{cc}
0 & I_{m} \\
-I_{m} & 0
\end{array}\right) \cdot \bar{y}
$$

is the standard anti-hermitian hyperbolic form and $M_{0}$ with its form ' $H_{0}$ is given as in the one of the following four cases:

- (a) $M_{0}=(0)$. (Here $n$ is even. If $n \geq 6$, the type is ${ }^{2} D_{n}^{\prime \prime}$.)

- (b) $M_{0}=\mathcal{O}(\mathcal{Q})$ with form $x c \bar{y}$ with $c=X, c=\zeta$, or $c=X \xi$. (Here $n$ is odd. The type is either ${ }^{2} D_{n}^{\prime \prime}$ if $c=\zeta$ and $n \geq 5$, or for $n \geq 3,{ }^{2} C-B_{n-1}$ otherwise.) 
- (c) $M_{0}=\mathcal{O}(\mathcal{Q})^{2}$ with form $x_{1} a_{1} \bar{y}_{1}+x_{2} a_{2} \bar{y}_{2}$ with $a_{1}, a_{2}$ two distinct elements of the set $\{X, \zeta, X \xi\}$. (Here $n$ is even. If $n \geq 4$, the type is ${ }^{4} D_{n}$.)

- (d) $M_{0}=\mathcal{O}(\mathcal{Q})^{3}$ with form $x_{1} X \bar{y}_{1}+x_{2} \zeta \bar{y}_{2}+x_{3} X \xi \bar{y}_{3}$. (Here $n$ is odd. If $n \geq 5$, the type is ${ }^{4} D_{n}$.)

4.c.9. Our list of cases above is exhaustive in the following sense: Choose once and for all the uniformizer $\varpi$ of $F$. The connected components of the specializations $\left.\mathcal{G}^{\prime}\right|_{W\left[v^{ \pm 1}\right]} \otimes_{W\left[v^{ \pm 1}\right]}$ $F$, together with the groups $\mathrm{SL}_{m}(D)$ for $F$-central division algebras $D$ (these groups are of type ${ }^{d} A_{m d-1}$ with $d$ the degree of $D$ ), give exactly all the isogeny classes of absolutely almost simple groups over $F$ which are of classical type. (More precisely, if we avoid exceptional isomorphisms by obeying the listed restrictions on $n$, we obtain each isogeny class exactly once.) This follows from the above, the discussion in [71, 4.5], and classical results on the classification of quadratic and (quaternionic) hermitian forms over local fields (e.g. [30], 72]). For example, to deal with the quasi-split case for symmetric forms, we notice that, since the residue characteristic is odd, $v \mapsto \varpi$ gives an isomorphism

$$
W\left[v^{ \pm 1}\right]^{*} /\left(W\left[v^{ \pm 1}\right]^{*}\right)^{2} \stackrel{\sim}{\rightarrow} F^{*} /\left(F^{*}\right)^{2} .
$$

This allows us to realize any quadratic extension $L / F$ as a specialization of a uniquely specified $\mathfrak{R} / W[v]$ at $v \mapsto \varpi$. As a result, the trace form $\frac{1}{2} \operatorname{Tr}_{L / F}($ ) can be obtained by specializing $\frac{1}{2} \operatorname{Tr}_{\mathfrak{R}\left[v^{ \pm 1}\right] / W\left[v^{ \pm}\right]}($) by $v \mapsto \varpi$.

4.c.10. In what follows, the symbol $\mathfrak{O}$ will denote either $\mathcal{O}(\mathcal{D})$ as in (4.5), or $W[v]$, $\mathfrak{R}$, $\mathcal{O}(\mathcal{Q})$ as in the previous sections. Recall that we denote by $\mathcal{G}^{\prime}$ the group scheme over $W[v]$ of $\mathfrak{O}$-automorphisms of $M$ that also preserve the form $h$ if applicable. All the above forms are "perfect" after we invert $v$, i.e over $W\left[v^{ \pm 1}\right]$, and we can see that $\left.\mathcal{G}^{\prime}\right|_{W\left[v^{ \pm 1}\right]}$ is reductive. Denote by $G^{\prime}$ the specialization of the neutral component $\left(\left.\mathcal{G}^{\prime}\right|_{W\left[v^{ \pm 1}\right]}\right)^{\circ} \otimes_{W\left[v^{ \pm 1}\right]} F$ and consider the Weil restriction of scalars $G=\operatorname{Res}_{F / \mathbb{Q}_{p}} G^{\prime}$. Regard $W\left[v^{ \pm 1}\right]$ as a $\mathbb{Z}_{p}\left[u^{ \pm 1}\right]$ algebra via $u \mapsto v^{e} \cdot p \varpi^{-e}$ as before.

Proposition 4.1. The group scheme $\underline{G}$, as constructed in 92.0 from $G$ above, is isomorphic to the neutral component of the group scheme over $\mathbb{Z}_{p}\left[u^{ \pm 1}\right]$ with $R$-valued points the $\mathfrak{O} \otimes_{\mathbb{Z}_{p}\left[u^{ \pm 1}\right]} R$-linear automorphisms of $M \otimes_{\mathbb{Z}_{p}\left[u^{ \pm 1}\right]} R$ that also respect the form $h \otimes_{\mathbb{Z}_{p}\left[u^{ \pm 1}\right]} R$ if applicable.

Proof. As above, consider the neutral component $\underline{J}:=\left(\left.\mathcal{G}^{\prime}\right|_{W\left[v^{ \pm 1}\right]}\right)^{\circ}$ of the group scheme over $W\left[v^{ \pm 1}\right]$ of $\mathfrak{O}$-automorphisms of $M$ that also preserve the form $h$ if applicable. Then the group scheme in the statement of the Proposition is isomorphic to $\operatorname{Res}_{W\left[v^{ \pm 1}\right] / \mathbb{Z}_{p}\left[u^{ \pm 1}\right]} \underline{J}$ and is enough to check that $\underline{J}$ is isomorphic to the group scheme $\underline{G}^{\prime}$ which is obtained by $G^{\prime}$ using our constructions in the previous chapters. This can be shown by a caseby-case verification and we will leave some of the work to the reader: First, in the case of inner forms of type $A_{n}$ where $\mathfrak{O}=\mathcal{O}(\mathcal{D})$, the result is in 4. .c.3 and follows directly from the construction of $42 . \mathrm{C}$. Second, suppose we consider the rest of the cases of the previous section; then we assume that $p$ is odd. The group $G^{\prime}$ contains a standard split torus and we can compute the quasi-split forms and the corresponding anisotropic kernel in the explicit descriptions of the cases above. The adjoint groups of these anisotropic 
kernels are inner forms of products of $\mathrm{PGL}_{2}$ or $\mathrm{PGL}_{4}$ (the latter occuring only in case 4. c.8 (d)). We first check that $\underline{J} \otimes_{W\left[u^{ \pm 1}\right]} \breve{\mathbb{Z}}_{p}\left[v^{ \pm 1}\right]$ is quasi-split over $\breve{\mathbb{Z}}_{p}\left[v^{ \pm 1}\right]$. Then the rigidity of quasi-split forms (\$2.3) shows the base change $\underline{J} \otimes_{W\left[u^{ \pm 1}\right]} \breve{\mathbb{Z}}_{p}\left[v^{ \pm 1}\right]$ is isomorphic to $\underline{G}^{*} \otimes_{W\left[u^{ \pm 1}\right]} \breve{\mathbb{Z}}_{p}\left[v^{ \pm 1}\right]$ and it remains to verify that the inner twists of $\underline{G}^{*}$ that define $\underline{J}$ and $\underline{G}^{\prime}$ agree. This can be done case-by-case; we leave the details to the reader. (Alternatively, since the reductive groups $\underline{J}$ always split over a degree 4 Galois cover of $W\left[u^{ \pm 1}\right]$ and $p$ is odd, the isomorphism between $\underline{J}$ and $\underline{G}^{\prime}$ can also be shown directly using Remark 2.2.)

4.c.11. We can now extend the explicit descriptions of the group schemes $\mathcal{G}$ from the split cases above to the general classical case. Recall $\mathfrak{O}$ will denote either $\mathcal{O}(\mathcal{D})$ as in (4.5), or $W[v], \mathfrak{R}, \mathcal{O}(\mathcal{Q})$ as in the previous sections. Then, we also denote by $Y$ the "standard uniformizer" of each of these algebras, i.e $X$ when $\mathfrak{O}$ is $\mathcal{O}(\mathcal{D})$ or $\mathcal{O}(\mathcal{Q}), v$ when $\mathfrak{O}=W[v]$ or $\mathfrak{R}=W_{2}[v]$ in the unramified case, $v^{\prime}$ when $\mathfrak{O}=\mathfrak{R}$ in the ramified case.

A $\mathfrak{O}$-lattice chain in $\mathfrak{O}\left[v^{ \pm 1}\right]^{n}$ is a totally ordered non-empty set $M_{\bullet}$ of left $\mathfrak{O}$-submodules of $\mathfrak{O}\left[v^{ \pm 1}\right]^{n}$ which are free of rank $n$, which is stable under multiplication by $Y$ and $Y^{-1}$, and of the form

$$
\cdots \subset Y M_{0} \subset M_{r-1} \subset \cdots \subset M_{1} \subset M_{0} \subset \cdots .
$$

with $M_{i} / M_{i+1}$, for all $i \in \mathbb{Z}$, free over $W$.

If $\mathfrak{O}$ is one of $W[v], \mathfrak{R}, \mathcal{O}(\mathcal{Q})$ with form $h$ as in the previous section, and $N$ a $\mathfrak{O}$-lattice in $\mathfrak{O}\left[v^{ \pm 1}\right]^{n}$ we can consider the dual $N^{\vee}=\left\{x \in \mathfrak{O}\left[v^{ \pm 1}\right]^{n} \mid h(x, m) \in \mathfrak{O}, \forall m \in N\right\}$ which is also an $\mathfrak{O}$-lattice. The $\mathfrak{O}$-lattice chain $M_{\bullet}$ is called self-dual, when $N$ belongs to the lattice chain $M_{\bullet}$, if and only if $N^{\vee}$ belongs to the lattice chain $M_{\bullet}$. Then, for each index $i$, there is $j$ such that $h$ induces a prefect pairing

$$
h: M_{i} \times M_{j} \rightarrow \mathfrak{O} .
$$

If $M_{\bullet}$ is a (self-dual) $\mathfrak{O}$-lattice chain, the base change $M_{\bullet} \otimes_{W[v]} \mathcal{O}$, by $v \mapsto \varpi$, gives a (self-dual) lattice chain as in $\$ 4 . \mathrm{a}$,

Remark 4.2. In fact, we can give such self-dual $\mathfrak{O}$-lattice chains by the following construction: Let $M$ be $\mathcal{O}(\mathcal{D})^{n}$ or in general $\mathfrak{D}^{n}$ with the form as in 4.c.5, 4.c.6, 4.c.7, etc. Consider the $F((v))$-vector space $V=M \otimes_{W\left[v^{ \pm 1}\right]} F((v))$; it is a free (left) $\mathfrak{O} \otimes_{W[v]} F((v))$ module and supports the perfect form $h \otimes_{W[v]} F((v))$. Choose a self-dual lattice chain $L^{\bullet}=\left(L_{i}\right)_{i}$ of $\mathfrak{O} \otimes_{W[v]} F[[v]]$-lattices in $V$ in the sense of $\$ 4 . a$ (cf. [55]) (for the equal characteristic dvr $F[[v]])$. Since $M \otimes_{W[v]} F[[v]]$ is also a lattice in $V$, for each $i \in \mathbb{Z}$, there is $r_{i} \geq 0$ so that $v^{r_{i}} M \otimes_{W[v]} F[[v]] \subset L_{i} \subset v^{-r_{i}} M \otimes_{W[v]} F[[v]]$. The intersection $M_{i}:=L_{i} \cap M\left[v^{-1}\right]$ is a finitely generated and reflexive (i.e equal to its double dual) $W[v]$-module. Since $W[v]$ is regular Noetherian of Krull dimension 2 and $(v, p)$ has codimension 2, it follows that $M_{i}$ is a finitely generated projective module which then is actually $W[v]$-free $([64])$. In fact, locally free coherent sheaves on $\operatorname{Spec}(W[v])-\{(v, p)\}$ uniquely extend to locally free coherent sheaves over $\operatorname{Spec}(W[v])$. Similarly, module homomorphisms between two such sheaves uniquely extend. Using this extension property, we now see that $\mathfrak{O}$-multiplication and the perfect forms (considered as maps $L_{i} \rightarrow L_{j}^{\text {dual }}$ ) 
extend to $\left\{M_{i}\right\}$. Suppose that $L_{i} \subset L_{j}$ are two $F[[v]]$-lattices in the chain and consider the corresponding $W[v]$-lattices $M_{i} \subset M_{j}$. Notice that $M_{i} / M_{j}$ has projective dimension 1 as a $W[v]$-module and so it follows from the Auslander-Buchsbaum theorem that $M_{i} / M_{j}$ has depth 1 . On the other hand, since $M_{i}\left[v^{-1}\right]=M_{j}\left[v^{-1}\right], M_{i} / M_{j}$ is supported along $v=0$. By the above, all the associated primes of $M_{i} / M_{j}$ have height 1 , therefore $M_{i} / M_{j}$ has no section supported over $(v, p)$. It now follows that if the quotient $L_{i} / L_{j}$ is annihilated by $v$ and has $F$-rank $d$, then $M_{i} / M_{j}$ is also annihilated by $v$ and is actually $W$-free of rank $d$. Similarly, we see that there are $m_{j} \geq 0$ such that

$$
v^{m_{j}} \mathfrak{O}^{n} \subset M_{i} \subset v^{-m_{j}} \mathfrak{O}^{n}
$$

with $M_{i} / v^{m_{j}} \mathfrak{O}^{n}, v^{-m_{j}} \mathfrak{O}^{n} / M_{i}$ both $W$-free. We can now show that all such $M_{i}$ are free $\mathfrak{O}$-modules. Hence, it also follows that $M_{\bullet}=\left\{M_{i}\right\}_{i}$ is a (self-dual) $\mathfrak{O}$-lattice chain in $M\left[u^{ \pm 1}\right]$ in the sense above.

Now consider the group scheme $\mathcal{G}^{\prime}$ over $\mathbb{Z}_{p}[u]$ with $R$-valued points the $\mathfrak{O} \otimes_{\mathbb{Z}_{p}[u]} R$ linear automorphisms of the chain $M_{\bullet} \otimes_{\mathbb{Z}_{p}[u]} R$ that respect the forms $h \otimes_{\mathbb{Z}_{p}[u]} R$ of (4.10). The arguments in [55, Appendix to Ch. 3] show that $\mathcal{G}^{\prime}$ is smooth. Base changing by $\mathbb{Z}_{p}[u] \rightarrow \mathbb{Z}_{p}$ via $u \mapsto p$ or by $\mathbb{Z}_{p}[u] \rightarrow \mathbb{Q}_{p}((u))$ produces smooth group schemes whose neutral component is a Bruhat-Tits parahoric group scheme (see \$4.a.2). Using 3.b.1, we can now see that the neutral component $\mathcal{G}$ of $\mathcal{G}^{\prime}$ is a group scheme obtained by Corollary 3.2 ,

4.c.12. Variants. Similarly, we can consider:

- anti-hermitian forms $\tilde{h}$ on $\mathfrak{R}^{n}$ obtained by multiplying the hermitian forms $h$ of 4.c.7 by either $\zeta$ (in the unramified case), or by $v^{\prime}$ (in the ramified cases).

- $\epsilon$-hermitian forms $\tilde{h}$ on $\mathcal{O}(\mathcal{D})$ for the "new involution" $d \mapsto d^{\prime}$ on $\mathcal{O}(\mathcal{D})$ given by $d^{\prime}:=X^{-1} \cdot \bar{d} \cdot X$. Such forms can be obtained by multiplying the $\epsilon$-hermitian forms $h$ for the main involution of $\$ 4 . c .8$ by $X$. Indeed, if $h$ is $\epsilon$-hermitian for $d \mapsto \bar{d}$, then $h \cdot X$ is $(-\epsilon)$-hermitian for $d \mapsto d^{\prime}$.

The automorphisms groups of these forms (after specialization to $F$ ) do not produce additional isogeny classes of reductive groups. However, as we will see later, considering these forms is useful in constructing certain symplectic embeddings and so these will appear in our discussion of local models for Shimura varieties of PEL type.

\section{LOOP GROUPS AND AFFInE GRASSMANNIANS}

In this chapter we define and show the (ind-)representability of the various versions ("local" and "global") of the affine Grassmannian that we will use. We start by showing a version of the descent lemma of Beauville-Laszlo for $\mathcal{G}$-torsors.

5.a. A descent lemma. We continue with the same notations, so that $\mathcal{O}$ is a discrete valuation ring with fraction field $F$ and perfect residue field $k$. Let $\mathcal{G}=\operatorname{Spec}(B) \rightarrow$ $X=\mathbb{A}_{\mathcal{O}}^{1}=\operatorname{Spec}(\mathcal{O}[u])$ be a smooth affine group scheme over $\mathbb{A}_{\mathcal{O}}^{1}$ with connected fibers. Suppose that $R$ is an $\mathcal{O}$-algebra and denote by $r: \operatorname{Spec}(R) \rightarrow \operatorname{Spec}(\mathcal{O}[u])$ the $R$-valued 
point of $\mathbb{A}_{\mathcal{O}}^{1}$ given by $u \mapsto r \in R$. We will identify the completion of $R[u]$ along the ideal $(u-r)$ (which cuts out the graph of $r$ ) with $R[[t]]$ using the local parameter $t=u-r$.

The following extends the descent lemma of Beauville-Laszlo [4].

Lemma 5.1. There is a 1-1 correspondence between elements of $\mathcal{G}(R((t)))$ and triples $(\mathcal{T}, \alpha, \beta)$, where $\mathcal{T}$ is a $\mathcal{G}$-torsor over $R[u]$ and $\alpha, \beta$ are trivializations of the torsors $\mathcal{T} \otimes_{R[u]} R\left[t, t^{-1}\right]$ and $\mathcal{T} \otimes_{R[u]} R[[t]]$ respectively. The inverse of the correspondence associates to the triple $(\mathcal{T}, \alpha, \beta)$ the element $\left(\alpha^{-1} \cdot \beta\right)(1)$.

Proof. In [4, this is proven when $\mathcal{G}=\mathrm{GL}_{n}$. More generally, 4] shows how one can construct a $t$-regular $R[u]$-module $M$ from a triple $(M, N, \phi)$ of a $R\left[t, t^{-1}\right]$-module $M$, a $t$-regular $R[[t]]$-module $N$ and an $R((t))$-isomorphism $\phi: R[[t]] \otimes_{R[u]} F \stackrel{\sim}{\rightarrow} N \otimes_{R[[t]]} R((t))$. Starting from $g \in \mathcal{G}(R((t)))$, we can apply this to $M=B \otimes_{R[u]} R\left[t, t^{-1}\right], N=B \otimes_{R[u]} R[[t]]$ and $\phi$ given by the (co)action of $g$, i.e $\phi$ is the composition

$$
B \otimes_{\mathcal{O}[u]} R((t)) \rightarrow\left(B \otimes_{\mathcal{O}[u]} R((t))\right) \otimes_{R((t))}\left(B \otimes_{\mathcal{O}[u]} R((t))\right) \stackrel{i d \otimes g^{*}}{\longrightarrow} B \otimes_{\mathcal{O}[u]} R((t)) .
$$

In this, $g^{*}$ is the $R[u]$-algebra homomorphism $B \rightarrow R((t))$ corresponding to $g \in \mathcal{G}(R((t)))$. Denote by $C$ the corresponding $R[u]$-module obtained using [4]. Notice that $\phi$ is an $R((t))$ algebra homomorphism; this allows us to deduce that $C$ is an $R[u]$-algebra. Since $B$ is flat over $\mathcal{O}[u]$, by [4] we see that $C$ is also flat over $R[u]$. In fact, since by construction, $C \otimes_{R[u]} R[[t]] \simeq B \otimes_{\mathcal{O}[u]} R[[t]]$, the map $\operatorname{Spec}(C) \rightarrow \operatorname{Spec}(R[u])$ is surjective and so $C$ is faithfully flat over $R[u]$. For our choice of $\phi$, the algebra $C$ affords a $B$-comodule structure $C \rightarrow B \otimes_{R[u]} C$ which base-changes to the standard $B$-comodule structures on $B \otimes_{\mathcal{O}[u]} R\left[t, t^{-1}\right]$ and $B \otimes_{\mathcal{O}[u]} R[[t]]$. Now set $\mathcal{T}=\operatorname{Spec}(C) \rightarrow \operatorname{Spec}(R[u])$. By the above, $\mathcal{T}$ is a faithfully flat scheme with $\mathcal{G}$-action which is $\mathcal{G}$-equivariantly isomorphic to $\mathcal{G}$ over $R[[t]]$ and $R\left[t, t^{-1}\right]$. We would like to conclude that $\mathcal{T}:=\operatorname{Spec}(C)$ is the corresponding torsor. Consider the map $m: \mathcal{G} \times_{\operatorname{Spec}(\mathcal{O}[u])} \mathcal{T} \rightarrow \mathcal{T} \times_{\operatorname{Spec}(R[u])} \mathcal{T}$ given by $(g, \tau) \mapsto(g \cdot \tau, \tau)$. It is enough to show that the corresponding ring homomorphism

$$
m^{*}: C \otimes_{R[u]} C \rightarrow B \otimes_{\mathcal{O}[u]} C
$$

is an isomorphism. Observe that $m^{*}$ is injective since $m^{*}\left[t^{ \pm 1}\right]$ is an isomorphism. Similarly, we can see that $m^{*}$ is surjective using [4, Lemme 2] since the base-change $R[u] \rightarrow$ $R\left[t, t^{-1}\right] \times R[[t]]$ is faithful and $m^{*}\left[t^{ \pm 1}\right], m^{*}[[t]]$ are isomorphisms.

\section{5.b. Affine Grassmannians.}

5.b.1. The local affine Grassmannian. If $S=\operatorname{Spec}(R)$ is an affine $\mathcal{O}$-scheme we set $D_{S}=$ $\operatorname{Spec}(R[[u]])$ and $D_{S}^{*}=D_{S}-\{0\}=\operatorname{Spec}(R((u)))$.

Let $\mathcal{G} \rightarrow X=\mathbb{A}_{\mathcal{O}}^{1}=\operatorname{Spec}(\mathcal{O}[u])$ be a smooth affine group scheme with connected fibers. If $R$ is an $\mathcal{O}$-algebra, we set $L \mathcal{G}(R)=\mathcal{G}(R((u)))$ and $L^{+} \mathcal{G}(R)=\mathcal{G}(R[[u]])$. Since $\mathcal{G}$ is affine, we can see that $L \mathcal{G}$, resp. $L^{+} \mathcal{G}$, is represented by an ind-affine scheme, resp. affine scheme, over $\mathcal{O}$. We also consider the quotient fpqc sheaf $\operatorname{Gr}_{\mathcal{G}}:=L \mathcal{G} / L^{+} \mathcal{G}$ on $(\mathrm{Sch} / \mathcal{O})$ associated to $R \mapsto L \mathcal{G}(R) / L^{+} \mathcal{G}(R)$. 
Proposition 5.2. If $S$ is an affine scheme over $\mathcal{O}$, there is a natural identification

$$
\operatorname{Gr}_{\mathcal{G}}(S)=\left\{\begin{array}{l|l}
\text { iso-classes of pairs }(\mathcal{T}, \alpha) & \begin{array}{c}
\mathcal{T} \text { a } \mathcal{G} \text {-torsor on } D_{S}, \\
\alpha \text { a trivialization of }\left.\mathcal{T}\right|_{D_{S}^{*}} ^{*}
\end{array}
\end{array}\right\}
$$

For any scheme $S$ over $\mathcal{O}$, there is a natural identification

$$
\operatorname{Gr}_{\mathcal{G}}(S)=\left\{\begin{array}{l|c}
\text { iso-classes of pairs }(\mathcal{E}, \beta) & \begin{array}{c}
\mathcal{E} \text { a } \mathcal{G} \text {-torsor on } \mathbb{A}_{S}^{1}, \\
\beta \text { a trivialization of }\left.\mathcal{E}\right|_{\mathbb{A}_{S}^{1} \backslash\{u=0\}} ^{1}
\end{array}
\end{array}\right\} .
$$

Proof. The argument showing this can be found in [42, see especially [42, Prop. 3.10]. The crucial point is to observe that every $\mathcal{G}$-torsor $\mathcal{T}$ over $R[[u]]$ can be trivialized over $R^{\prime}[[u]]$ where $R \rightarrow R^{\prime}$ is a faithfully flat extension. Indeed, $\mathcal{T} \otimes_{R[[u]]} R$ has a section after such an extension $R \rightarrow R^{\prime}$; since $\mathcal{T} \rightarrow \operatorname{Spec}(R[[u]])$ is smooth this section can be extended to a section over $R^{\prime}[[u]]$. This shows the first identification. The second identification now follows using the descent lemma 5.1 .

5.b.2. Assume now in addition that $\mathcal{G}=\mathcal{G}_{x} \rightarrow X=\mathbb{A}_{\mathcal{O}}^{1}=\operatorname{Spec}(\mathcal{O}[u])$ is a Bruhat-Tits group scheme in the sense of Theorem 3.1 .

Proposition 5.3. The sheaf $\mathrm{Gr}_{\mathcal{G}}$ is represented by an ind-projective ind-scheme over $\mathcal{O}$.

Proof. For this we can appeal to the sketchy [16] (for the split case) and to [49] when the residue field $k$ is algebraically closed. We give here a general proof by a different argument.

We first show that $\mathrm{Gr}_{\mathcal{G}}$ is representable by an ind-scheme of ind-finite type and separated over $X$. By Proposition 10.7, there is a closed group scheme immersion $\mathcal{G} \hookrightarrow \mathrm{GL}_{n}$ such that the quotient $\mathrm{GL}_{n} / \mathcal{G}$ is representable by a quasi-affine scheme over $\mathcal{O}[u]$. The argument in [5, 4.5.1] (or [17, Appendix], cf. [49]) now shows that the natural functor $\mathrm{Gr}_{\mathcal{G}} \rightarrow \mathrm{Gr}_{\mathrm{GL}_{n}}$ is representable and is a locally closed immersion. In fact, if the quotient $\mathrm{GL}_{n} / \mathcal{G}$ is affine, this functor is a closed immersion. Now note that, as is well-known (loc. cit.), the affine Grassmannian $\mathrm{Gr}_{\mathrm{GL}_{n}}$ is representable by an ind-scheme which is ind-projective over $\mathcal{O}$. It remains to show that $\mathrm{Gr}_{\mathcal{G}}$ is ind-proper.

Assume first that $\underline{G}=H \otimes_{\mathbb{Z}} \mathcal{O}\left[u^{ \pm 1}\right]$ is split. Consider an alcove $C$ whose closure contains $x$. If $y$ is in the interior of the alcove $C$, then an argument as in 3.b.1 shows that there is a group scheme homomorphism $\mathcal{G}_{y} \rightarrow \mathcal{G}_{x}$ which induces $\mathcal{G}_{y}\left[u^{-1}\right]=\mathcal{G}_{x}\left[u^{-1}\right]$. ( $\mathcal{G}_{y}$ is a group scheme corresponding to an Iwahori subgroup.) Hence, the morphism $\mathrm{Gr}_{\mathcal{G}_{y}}=L \mathcal{G}_{y} / L^{+} \mathcal{G}_{y} \rightarrow \operatorname{Gr}_{\mathcal{G}_{x}}=L \mathcal{G}_{x} / L^{+} \mathcal{G}_{x}$ is surjective and it is enough to show that $\operatorname{Gr}_{\mathcal{G}_{y}}$ is ind-proper. Now observe that the closure of the alcove $C$ always contain a hyperspecial point $x_{0}$; then $\mathcal{G}_{x_{0}}$ is reductive, $\mathcal{G}_{x_{0}} \simeq H \otimes_{\mathbb{Z}} \mathcal{O}[u]$. As in the proof of Theorem 3.1 the group scheme homomorphism $\mathcal{G}_{y} \rightarrow \mathcal{G}_{x_{0}}$ identifies $\mathcal{G}_{y}$ with the dilatation of $H \otimes_{\mathbb{Z}} \mathcal{O}[u]$ along a Borel subgroup $B$ of the fiber $H$ over $u=0$. This implies that the fpqc sheaf associated to $R \mapsto \mathcal{G}_{x_{0}}(R[[u]]) / \mathcal{G}_{y}(R[[u]])$ is representable by the smooth projective homogeneous space $Y:=H / B$ over $\mathcal{O}$. Hence, the morphism $\mathrm{Gr}_{\mathcal{G}_{y}}=L \mathcal{G}_{y} / L^{+} \mathcal{G}_{y} \rightarrow \mathrm{Gr}_{\mathcal{G}_{x_{0}}}=L \mathcal{G}_{x_{0}} / L^{+} \mathcal{G}_{x_{0}}$ is an fppf fibration with fibers locally isomorphic to $Y$; in particular it is a projective surjective morphism. (In fact, we note here that, as in [16], we can see that the quotient morphism $L \mathcal{G}_{y} \rightarrow \operatorname{Gr}_{\mathcal{G}_{y}}=L \mathcal{G}_{y} / L^{+} \mathcal{G}_{y}$ is an $L^{+} \mathcal{G}_{y}$-torsor which splits locally for the Zariski 
topology.) Now recall that there is a representation $H \hookrightarrow \mathrm{GL}_{n}$ with $\mathrm{GL}_{n} / H$ affine, while $\mathcal{G}_{x_{0}} \simeq H \otimes_{\mathbb{Z}} \mathcal{O}[u]$. As above, we see then that $\operatorname{Gr}_{\mathcal{G}_{x_{0}}} \hookrightarrow \mathrm{Gr}_{\mathrm{GL}_{n}}$ is a closed immersion, and that $\mathrm{Gr}_{\mathcal{G}_{x_{0}}}$ is ind-projective and also ind-proper.

Next we consider the general case. It is enough to prove that $\operatorname{Gr}_{\mathcal{G}}$ is ind-proper over $\operatorname{Spec}(\mathcal{O})$ after base changing by a finite unramified extension $\mathcal{O}^{\prime} / \mathcal{O}$. Therefore, by replacing $\mathcal{O}$ by $\mathcal{O}^{\prime}$ we may assume that $\underline{G}$ is quasi-split and splits over $\mathcal{O}\left[v^{ \pm 1}\right] / \mathcal{O}\left[u^{ \pm 1}\right]$. We now return to the notations of the proof of Theorem 3.1. In particular, if $x$ is in $\mathcal{A}\left(\underline{G}_{F}, \underline{S}_{F}, F\right)=\mathcal{A}\left(H, T_{H}, \tilde{F}\right)^{\Gamma}$, then $\mathcal{G}_{x}$ is the neutral component of $\left(\operatorname{Res}{ }_{\mathcal{O}[v] / \mathcal{O}[u]} \mathcal{H}_{x}\right)^{\gamma_{0}}$. By the argument in the last part of that proof, we can find a $\gamma_{0}$-stable affine alcove $C$ in the apartment $\mathcal{A}\left(H_{\tilde{F}}, T_{H}, \tilde{F}\right)$ such that $x$ belongs to the closure $\bar{C}$. Denote by $y$ the barycenter of $C$ which is then fixed by $\gamma_{0}$. Then $\mathcal{H}_{y}$ is an Iwahori group scheme and $\overline{\mathcal{H}}_{y}^{\text {red }}=\mathcal{T}$ is the split torus over $\mathcal{O}$. An argument as in the split case above, shows that it is enough to show that $\operatorname{Gr}_{\mathcal{G}_{y}}$ is ind-proper. For simplicity, set $\mathcal{G}=\mathcal{G}_{y}, \mathcal{H}=\mathcal{H}_{y}$. There is an exact sequence of pointed sets

$$
\mathcal{H}(R((v)))^{\gamma_{0}} / \mathcal{H}(R[[v]])^{\gamma_{0}} \hookrightarrow\left(\mathcal{H}(R((v))) / \mathcal{H}(R[[v]])^{\gamma_{0}} \stackrel{\delta}{\rightarrow} \mathrm{H}^{1}(\Gamma, \mathcal{H}(R[[v]])) .\right.
$$

Now observe $\left(\mathcal{H}(R((v))) / \mathcal{H}(R[[v]])^{\gamma_{0}}=\operatorname{Gr}_{\mathcal{H}}(R)^{\gamma_{0}}=\operatorname{Gr}_{\mathcal{H}}^{\gamma_{0}}(R)\right.$, where $\operatorname{Gr}_{\mathcal{H}}^{\gamma_{0}}$ is the closed ind-subscheme of $\operatorname{Gr}_{\mathcal{H}}$ given by taking $\gamma_{0}$-fixed points. The kernel of $\left.\mathcal{H}(R[[v]])\right) \rightarrow$ $\overline{\mathcal{H}}^{\text {red }}(R)=\mathcal{T}(R)$ is affine pro-unipotent and we see that $\mathrm{H}^{1}(\Gamma, \mathcal{H}(R[[v]]))=\mathrm{H}^{1}(\Gamma, \mathcal{T}(R))$. Now, consider the closed subgroup scheme $Q_{\mathcal{T}}$ of $\mathcal{T}$ of elements $x$ that satisfy the equation $N(x)=\prod_{i=0}^{e-1} \gamma_{0}^{i}(x)=1$. We can see that the sheaf $R \mapsto \mathrm{H}^{1}(\Gamma, \mathcal{T}(R))$ is given by the quotient $Q_{\mathcal{T}} / \mathcal{T}^{\gamma_{0}-1}$. The map $\delta$ is given as follows: starting with $x \in\left(\mathcal{H}(R((v))) / \mathcal{H}(R[[v]])^{\gamma_{0}}\right.$ we can find $h \in \mathcal{H}(R((u)))$ such that $h \gamma_{0}(h)^{-1}$ is in $\left.\mathcal{H}(R[[v]])\right)$. We set $\delta(x)=\overline{h \gamma_{0}(h)^{-1}}$ which is well-defined in $Q_{\mathcal{T}} / \mathcal{T}^{\gamma_{0}-1}$. Using Proposition 1.4 we see that $Q_{\mathcal{T}} / \mathcal{T}^{\gamma_{0}-1}$ is a finite étale commutative group scheme $Q$ over $\mathcal{O}$ (of order that divides $e$ ). The above exact sequence now gives that the sheaf associated to the presheaf $R \mapsto \mathcal{H}(R((v)))^{\gamma} / \mathcal{H}(R[[v]])^{\gamma_{0}}$ is represented by the fiber of the ind-scheme morphism $\delta: \operatorname{Gr}_{\mathcal{H}}^{\gamma_{0}} \rightarrow Q$ over the identity section $\operatorname{Spec}(\mathcal{O}) \rightarrow Q$. We conclude that the fpqc quotient $L \mathcal{H}^{\gamma_{0}} / L^{+} \mathcal{H}^{\gamma_{0}}$ is represented by an ind-proper ind-scheme over $\mathcal{O}$. To finish the proof recall that by construction $\mathcal{G}$ is the neutral component of $\left(\operatorname{Res}_{\mathcal{O}[v] / \mathcal{O}[u]} \mathcal{H}\right)^{\gamma_{0}}$. Using this, Corollary 1.4 and the fact that $\gamma_{0}$-fixed points of affine pro-unipotent groups are connected, we see that the sheaf associated to

$$
R \rightarrow\left(\operatorname{Res}_{\mathcal{O}[v] / \mathcal{O}[u]} \mathcal{H}\right)^{\gamma_{0}}(R[[u]]) / \mathcal{G}(R[[u]])=\mathcal{H}(R[[v]])^{\gamma_{0}} / \mathcal{G}(R[[u]])
$$

is represented by the finite étale commutative group scheme of connected components of $\mathcal{T}=\overline{\mathcal{H}}^{\text {red }}$. Therefore, $\operatorname{Gr}_{\mathcal{G}}$ given by $R \mapsto \mathcal{H}(R((v)))^{\gamma_{0}} / \mathcal{G}(R[[u]])=\mathcal{G}(R((u))) / \mathcal{G}(R[[u]])$ is represented by a finite étale cover of $L \mathcal{H}^{\gamma_{0}} / L^{+} \mathcal{H}^{\gamma_{0}}$. As such it is also an ind-proper ind-scheme over $\mathcal{O}$.

5.b.3. The global affine Grassmannian. We continue with the same assumptions, but for a little while we allow $\mathcal{G}$ to be any smooth affine group scheme over $X=\mathbb{A}_{\mathcal{O}}^{1}$ with connected fibers. 
Let $S \in(\mathrm{Sch} / X)$, with structure morphism $y: S \rightarrow X$. We will denote by $\Gamma_{y} \subset X \times S$ the closed subscheme given by the graph of $y$ and consider the formal completion of $X \times S$ along $\Gamma_{y}$. Suppose that $S=\operatorname{Spec}(R)$ is affine. Then the above completion is an affine formal scheme and following [5, 2.12] we can also consider the affine scheme $\hat{\Gamma}_{y}$ given by the relative spectrum of the ring of regular functions on that completion. There is a natural closed immersion $\Gamma_{y} \rightarrow \hat{\Gamma}_{y}$ and we will denote by $\hat{\Gamma}_{y}^{\circ}:=\hat{\Gamma}_{y}-\Gamma_{y}$ the complement of the image. If $y: \operatorname{Spec}(R) \rightarrow X=\mathbb{A}_{\mathcal{O}}^{1}$ is given by $u \mapsto y$, we have $\Gamma_{y} \simeq \operatorname{Spec}(R[u] /(u-y))$, $\hat{\Gamma}_{y} \simeq \operatorname{Spec}(R[[w]])$. When $y=0, \hat{\Gamma}_{y}=D_{S}, \hat{\Gamma}_{y}^{\circ}=D_{S}^{*}$ as before. We can see directly that there is a morphism $\hat{\Gamma}_{y} \rightarrow X \times S$ given by $R[u] \rightarrow R[[w]] ; u \mapsto w+y$. We will often write $\hat{\Gamma}_{y}=\operatorname{Spec}(R[[u-y]])$. Then $\hat{\Gamma}_{y}^{\circ}=\operatorname{Spec}\left(R[[u-y]]\left[(u-y)^{-1}\right]\right)$.

5.b.4. We will now consider various functors on $(\mathrm{Sch} / X)$. These will be fpqc sheaves on $X$ that can be described by giving their values on affine schemes over $X$.

First consider the functor that associates to an $\mathcal{O}[u]$-algebra $R$ (given by $u \mapsto y$ ) the group

$$
\mathcal{L G}(R)=\mathcal{G}\left(\hat{\Gamma}_{y}^{\circ}\right)=\mathcal{G}\left(R[[u-y]]\left[(u-y)^{-1}\right]\right) .
$$

Since $\mathcal{G} \rightarrow \operatorname{Spec}(\mathcal{O}[u])$ is smooth and affine, $\mathcal{L G}$ is represented by a formally smooth ind-scheme over $X$.

Next consider the functor that associates to an $\mathcal{O}[u]$-algebra $R$ the group

$$
\mathcal{L}^{+} \mathcal{G}(R)=\mathcal{G}\left(\hat{\Gamma}_{y}\right)=\mathcal{G}(R[[u-y]]) .
$$

We can see that $\mathcal{L}^{+} \mathcal{G}$ is represented by a scheme over $X$ (not of finite type) which is formally smooth.

Finally define the global affine Grassmannian of $\mathcal{G}$ over $X$ to be the functor on $(\mathrm{Sch} / X)$ given by

$$
\operatorname{Gr}_{\mathcal{G}, X}(S)=\left\{\begin{array}{l|l}
\text { iso-classes of pairs }(\mathcal{E}, \beta) & \begin{array}{c}
\mathcal{E} \text { a } \mathcal{G} \text {-torsor on } X \times S, \\
\beta \text { a trivialization of }\left.\mathcal{E}\right|_{(X \times S) \backslash \Gamma_{y}}
\end{array}
\end{array} .\right.
$$

Here and everywhere else the fiber products are over $\operatorname{Spec}(\mathcal{O})$.

Similarly to Proposition 5.2 , the descent lemma 5.1 implies that for $S=\operatorname{Spec}(R)$ the natural map given by restriction along $\hat{\Gamma}_{y} \rightarrow X \times S$

$$
\operatorname{Gr}_{\mathcal{G}, X}(R) \rightarrow\left\{\begin{array}{l|l}
\text { iso-classes of pairs }(\mathcal{E}, \beta) & \begin{array}{c}
\mathcal{E} \text { a } \mathcal{G} \text {-torsor on } \hat{\Gamma}_{y}, \\
\beta \text { a trivialization of }\left.\mathcal{E}\right|_{\hat{\Gamma}_{y}^{\circ}}
\end{array}
\end{array}\right\}
$$

is a bijection for each $\mathcal{O}[u]$-algebra $R$. This provides with an alternative description of $\operatorname{Gr}_{\mathcal{G}, X}$. Using this description, we can see that $\mathcal{L} \mathcal{G}, \mathcal{L}^{+} \mathcal{G}$ act on $\operatorname{Gr}_{\mathcal{G}, X}$ by changing the trivialization $\beta$. In fact, we have $\operatorname{Gr}_{\mathcal{G}, X} \simeq \mathcal{L} \mathcal{G} / \mathcal{L}^{+} \mathcal{G}$ but we are not going to use this.

5.b.5. Suppose now that $\mathcal{G}$ is as in Theorem 3.1. Let $\kappa$ be either the fraction field $F$ or the residue field $k$ of $\mathcal{O}$. Let $x: \operatorname{Spec}(\kappa) \rightarrow X$, where $\kappa$ is as above and identify the completed local ring $\widehat{\mathcal{O}}_{x}$ of $X \times \operatorname{Spec}(\kappa)$ with $\kappa[[t]]$, using the local parameter $t=u-x$. Let

$$
\mathcal{G}_{\kappa, x}:=\mathcal{G} \times_{\operatorname{Spec}(\mathcal{O}[u])} \operatorname{Spec}(\kappa[[t]])
$$


(i) Suppose that $x$ factors through $0: \operatorname{Spec}(\mathcal{O}) \rightarrow X$. Recall that by Theorem 3.1 the base change $\mathcal{G}_{\kappa, 0}$, can be identified with a Bruhat-Tits group scheme $P_{\kappa}:=\mathcal{P}_{x_{\kappa((u))}}$ over the $\operatorname{dvr} \kappa[[t]]=\kappa[[u]]$.

Let $L^{+} P_{\kappa}$ be the affine group scheme over $\operatorname{Spec}(\kappa)$ representing the functor on $\kappa$ algebras

$$
R \mapsto L^{+} P_{\kappa}(R)=P_{\kappa}(R[[t]]),
$$

and $L P_{\kappa}$ the ind-group scheme over $\operatorname{Spec}(\kappa)$ representing the functor

$$
R \mapsto L P_{\kappa}(R)=P_{\kappa}(R((t)))=G_{\kappa}(R((t))) .
$$

Here $G_{\kappa}=P_{\kappa}\left[t^{-1}\right]$ (which is denoted by $\underline{G}_{\kappa((u) \text { ) }}$ in Chapter 3 ) is the connected reductive group over $\kappa((t))$ which is obtained by base changing $\mathcal{G} \rightarrow \operatorname{Spec}(\mathcal{O}[u])$ along $\mathcal{O}[u] \rightarrow$ $\kappa((t)), u \mapsto t$. As in Proposition 5.3 we see that there is an ind-proper ind-scheme $\mathrm{Gr}_{P_{\kappa}}$ over $\kappa$ which represents the quotient $L P_{\kappa} / L^{+} P_{\kappa}$ of fpqc-sheaves on $\kappa$-schemes. By Proposition 5.2, $\operatorname{Gr}_{P_{\kappa}}$ is the ind-scheme representing

$$
R \mapsto \operatorname{Gr}_{P_{\kappa}}(R)=\left\{\begin{array}{l|l}
\text { iso-classes of pairs }(\mathcal{E}, \beta) & \begin{array}{c}
\mathcal{E} \text { a } P_{\kappa} \text {-torsor on } \operatorname{Spec} R[[t]], \\
\beta \text { a trivialization of }\left.\mathcal{E}\right|_{\operatorname{Spec} R((t))}
\end{array}
\end{array}\right\} .
$$

The base change $\operatorname{Gr}_{P_{\kappa}} \times_{\operatorname{Spec}(\kappa)} \operatorname{Spec}(\bar{\kappa})$ is an affine flag variety as in [49].

(ii) Suppose $x: \operatorname{Spec}(\kappa) \rightarrow X$ does not factor through $0: \operatorname{Spec}(\mathcal{O}) \rightarrow X$. Then by Theorem 3.1 (i), the base change $\mathcal{G}_{\kappa, x}$ is a reductive group scheme which is a form of $H$. We can see that

$$
\mathcal{G}_{\kappa, x} \times_{\operatorname{Spec}(\kappa)} \operatorname{Spec}\left(\kappa^{\prime}\right) \simeq H \times_{\operatorname{Spec}(\mathcal{O})} \operatorname{Spec}\left(\kappa^{\prime}[[t]]\right)
$$

for a finite $\kappa^{\prime} / \kappa$. As above, we also have the affine Grassmannian $\operatorname{Gr}_{\mathcal{G}_{\kappa, x}}$ over $\kappa$; by the above, we can see that $\operatorname{Gr}_{\mathcal{G}_{\kappa, x}} \times{ }_{\operatorname{Spec}(\kappa)} \operatorname{Spec}(\bar{\kappa})$ can be identified with the usual affine Grassmannian $\operatorname{Gr}_{H}$ over $\bar{\kappa}$ for the split reductive group $H$. The following observation now follows from Proposition 5.2 .

Proposition 5.4. Let $x: \operatorname{Spec}(\kappa) \rightarrow X$, where $\kappa$ is either the residue field $k$ of $\mathcal{O}$, or the fraction field $F$ of $\mathcal{O}$, and identify the completed local ring $\widehat{\mathcal{O}}_{x}$ of $X \times \operatorname{Spec}(\kappa)$ with $\kappa[[t]]$, using the local parameter $t=u-x$. Then restricting $\mathcal{G}$-bundles from $\mathcal{O}[u] \otimes_{\mathcal{O}} R$ to $R[[t]], u \mapsto t+x$, induces an isomorphism over $\operatorname{Spec}(\kappa)$,

$$
i_{x}^{*}: \operatorname{Gr}_{\mathcal{G}, X} \times{ }_{X, x} \operatorname{Spec}(\kappa) \stackrel{\sim}{\rightarrow} \operatorname{Gr}_{\mathcal{G}_{\kappa, x}} .
$$

Here $\operatorname{Gr}_{\mathcal{G}_{\kappa, x}}=L \mathcal{G}_{\kappa, x} / L^{+} \mathcal{G}_{\kappa, x}$ denotes the affine Grassmannian over $\kappa$ as above; this is isomorphic to either $\mathrm{Gr}_{P_{\kappa}}$ if $x$ maps to 0 , or to $\mathrm{Gr}_{H}$ over $\bar{\kappa}$ otherwise.

Notice here that at this point we only consider $\operatorname{Gr}_{\mathcal{G}, X}$ as a fpqc sheaf over $X$. However, using the next proposition we will soon see that these are actually isomorphisms of indschemes. Remark here that the above proposition combined with Proposition 5.3 already shows that the fiber of $\operatorname{Gr}_{\mathcal{G}, X}$ over $x: \operatorname{Spec}(\kappa) \rightarrow X$ is represented by an ind-scheme which is ind-projective over $\operatorname{Spec}(\kappa)$.

Proposition 5.5. Suppose that $\mathcal{G}$ is as in Theorem 3.1. The functor $\operatorname{Gr}_{\mathcal{G}, X}$ on $(\mathrm{Sch} / X)$ is representable by an ind-projective ind-scheme over $X$. 
Proof. We first show that $\mathrm{Gr}_{\mathcal{G}, X}$ is representable by an ind-scheme of ind-finite type and separated over $X$. This follows the corresponding argument in the proof of Proposition 5.3. By Proposition 10.7, there is a closed group scheme immersion $\mathcal{G} \hookrightarrow \mathrm{GL}_{n}$ such that the quotient $\mathrm{GL}_{n} / \mathcal{G}$ is representable by a quasi-affine scheme over $\mathcal{O}[u]$. The argument in [5] (or [17, Appendix]) now shows that the natural functor $\operatorname{Gr}_{\mathcal{G}, X} \rightarrow \mathrm{Gr}_{\mathrm{GL}_{n}, X}$ is representable and is a locally closed immersion. In fact, if the quotient $\mathrm{GL}_{n} / \mathcal{G}$ is affine, this functor is a closed immersion. Now note that $\operatorname{Gr}_{\mathrm{GL}_{n}, X}$ is representable by an indscheme separated of ind-finite type over $X$. This is well-known (see for example [5]). In fact, $\mathrm{Gr}_{\mathrm{GL}_{n}, X}$ is ind-projective over $X$.

It remains to show that $\operatorname{Gr}_{\mathcal{G}, X} \rightarrow X$ is ind-proper. By Propositions 5.3 and 5.4 each fiber of $\operatorname{Gr}_{\mathcal{G}, X} \rightarrow X$ is ind-proper. It is enough to show that the base change by $\tilde{X}=\operatorname{Spec}\left(\tilde{\mathcal{O}}_{0}[v]\right) \rightarrow X$ is ind-proper. Notice that, since $\tilde{X}-\{0\} \rightarrow X-\{0\}$ is finite étale, there is an isomorphism $\operatorname{Gr}_{\mathcal{G}, X} \times_{X}(\tilde{X}-\{0\}) \simeq \operatorname{Gr}_{H, X} \times_{X}(\tilde{X}-\{0\})=\operatorname{Gr}_{H} \times_{\mathcal{O}}(\tilde{X}-\{0\})$ (cf. [76, Lemma 3.3], here again $H$ is the split Chevalley form). Therefore, by Proposition 5.3 applied to $\operatorname{Gr}_{H}$, we see that the restriction of $\operatorname{Gr}_{\mathcal{G}, X} \rightarrow X$ over $U=(\tilde{X}-\{0\}) \otimes_{\mathcal{O}} F$ is ind-proper. We can write this restriction as a limit $S_{i}$ of proper schemes over $U$. In fact, using standard results on the structure of the affine Grassmannians $\operatorname{Gr}_{H}$ over the field $F\left([17]\right.$, [16], 49]) we can assume that $S_{i}=\sqcup_{j} S_{i j}$ with $S_{i j}$ proper schemes over $U$ with geometrically connected fibers. Denote by $Y_{i j}$, resp. $Z_{i j}$, the Zariski closures of $S_{i j}$ in $\operatorname{Gr}_{\mathcal{G}, X} \times_{X} \tilde{X}$, resp. $\operatorname{Gr}_{\mathrm{GL}_{n}, X} \times_{X} \tilde{X}$. Since $\mathrm{Gr}_{\mathrm{GL}_{n}, X} \rightarrow X$ is ind-proper, $Z_{i j} \rightarrow \tilde{X}$ is proper. Since $\mathrm{Gr}_{\mathcal{G}, X} \rightarrow \mathrm{Gr}_{\mathrm{GL}_{n}, X}$ is a locally closed immersion, $Y_{i j}$ is open and dense in $Z_{i j}$. Denote by bar fibers at a closed point of $\tilde{X}$. It enough to show that we always have $\bar{Y}_{i j}=\bar{Z}_{i j}$. Since all the fibers of $\operatorname{Gr}_{\mathcal{G}, X} \rightarrow X$ are ind-proper, $\bar{Y}_{i j}$ is proper and so $\bar{Y}_{i j}$ is closed in $\bar{Z}_{i j}$. By Zariski's main theorem applied to $Z_{i j} \rightarrow \tilde{X}$, we see that $\bar{Z}_{i j}$ is connected and so $\bar{Y}_{i j}=\bar{Z}_{i j}$. Hence, $Y_{i j}=Z_{i j}$ and $Y_{i j} \rightarrow \tilde{X}$ is proper. It remains to see that each point of each fiber of $\operatorname{Gr}_{\mathcal{G}, X} \times_{X} \tilde{X} \rightarrow \tilde{X}$ belongs to some $Y_{i j}$. This lifting property can be seen by the argument in the proof of Proposition 8.6.

5.b.6. Specialization along $u=\varpi$. Now let us fix a uniformizer $\varpi$ of $\mathcal{O}$. We denote by $\varpi$ the section of $X=\mathbb{A}_{\mathcal{O}}^{1}$ over $\mathcal{O}$ defined by $u \mapsto \varpi$. Let $G$ be connected reductive over $F$, and split over a tamely ramified extension $\tilde{F} / F$ as in 2.a. let $\underline{G}$ be constructed from $G$ as in \2. In addition, we fix an isomorphism $\underline{G}_{F} \simeq G$ from a rigidification of $G$ as explained in 3.c.1. This produces a group scheme $\mathcal{G}:=\mathcal{G}_{x}$ as in Corollary 3.2, which is independent of the choice of the rigidification of $G$ up to isomorphism.

Notice that there is an isomorphism

$$
\tilde{\mathcal{O}}_{0}\left[v^{ \pm 1}\right] \otimes_{\mathcal{O}\left[u^{ \pm 1}\right]} F[[u-\varpi]] \stackrel{\sim}{\rightarrow} \tilde{F}[[z]]=\tilde{F}[[u-\varpi]],
$$

given by $v \mapsto \tilde{\varpi} \cdot(1+z)$ where $\tilde{\varpi}^{e}=\varpi$. Here $z$ maps to the power series $\left(1+\frac{(u-\varpi)}{\varpi}\right)^{1 / e}-1$, where the $e$-th root is expressed by using the standard binomial formula. This isomorphism matches the action of $\Gamma$ on the left hand side (coming from the cover $\mathcal{O}[u] \rightarrow \tilde{\mathcal{O}}_{0}[v]$ by base change), with the action on $\tilde{F}[[z]]$ given by the Galois action on the coefficients $\tilde{F}$. Using this and the construction of the group scheme $\underline{G}$ in $\$ 2$ we obtain an isomorphism

$$
\mathcal{G}_{F, \varpi} \stackrel{\sim}{\rightarrow} G \times \times_{\operatorname{Spec}(F)} \operatorname{Spec}(F[[u-\varpi]])
$$


well defined up to $G(F)$-conjugation.

Denote by $\mathrm{Gr}_{\mathcal{G}, \mathcal{O}}$ the fiber product

$$
\operatorname{Gr}_{\mathcal{G}, \mathcal{O}}:=\operatorname{Gr}_{\mathcal{G}, X} \times_{X, \varpi} \operatorname{Spec}(\mathcal{O}) \rightarrow \operatorname{Spec}(\mathcal{O}) .
$$

Using Proposition [5.5 we see that this is an ind-projective ind-scheme over $\operatorname{Spec}(\mathcal{O})$.

Proposition 5.4 and the discussion in the beginning of 5. b.5 implies:

Corollary 5.6. 1) The generic fiber $\operatorname{Gr}_{\mathcal{G}, \mathcal{O}} \times_{\operatorname{Spec}(\mathcal{O})} \operatorname{Spec}(F)$ is equivariantly isomorphic to the affine Grassmannian $\operatorname{Gr}_{G, F}$ of $G$ over $\operatorname{Spec}(F)$.

2) The special fiber $\operatorname{Gr}_{\mathcal{G}, \mathcal{O}} \times{ }_{\operatorname{Spec}(\mathcal{O})} \operatorname{Spec}(k)$ is equivariantly isomorphic to the affine Grassmannian $\operatorname{Gr}_{P_{k}}$ over $\operatorname{Spec}(k)$.

5.b.7. Notation. Let $\mathcal{G}$ be as in Corollary 3.2. If $f: S=\operatorname{Spec}(R) \rightarrow X$ is a scheme morphism given by $u \mapsto r$, we will write $\operatorname{Gr}_{\mathcal{G}, R, r}$ for the fiber product $\operatorname{Gr}_{\mathcal{G}, X} \times_{X, f} S$. If $R$ is an $\mathcal{O}$-algebra and $\mathcal{O}[u] \rightarrow R$ is given by $u \mapsto \varpi$, we will simple write $\operatorname{Gr}_{\mathcal{G}, R}$ instead. This agrees with our use of the notation $\operatorname{Gr}_{\mathcal{G}, \mathcal{O}}$ above.

\section{LOCAL MODELS}

Here we give our group-theoretic definition of local models. We also explain how, in the examples of $\mathrm{GL}_{n}, \mathrm{GSp}_{2 n}$ and a minuscule coweight, it follows from [19] and [20] that these agree with the local models of [55]. This last result will be generalized in the next chapter.

\section{6.a. Generalized local models.}

6.a.1. Cocharacters. We continue with the above assumptions and notations. Suppose now that $\{\mu\}$ is a geometric conjugacy class of one parameter subgroups of $G$, defined over an algebraic closure $\bar{F}$ of $F$ that contains the field $\tilde{F}$. Let $E$ be the field of definition of $\{\mu\}$, a finite extension of $F$ contained in $\bar{F}$ (the reflex field of the pair $(G,\{\mu\})$ ).

First observe that since $G$ is quasi-split over the maximal unramified extension $\tilde{F}_{0}$ of $F$ in $\tilde{F}$ we can find ([34, Lemma (1.1.3)]) a representative of $\{\mu\}$ defined over $E^{\prime}=E \tilde{F}_{0}$, which factors $\mu: \mathbb{G}_{\mathrm{m} E^{\prime}} \rightarrow T_{E^{\prime}} \rightarrow G_{E^{\prime}}$, where $T$ is the maximal torus of $G$ given as in 1.d.2. Notice that $\mu$ gives an $E^{\prime}\left[z, z^{-1}\right]$-valued point of $G_{E^{\prime}}$, therefore an $E^{\prime}((z))$-valued point of $G_{E^{\prime}}$, therefore an $E^{\prime}$-valued point of the loop group $L G$. By (5.10) we have an isomorphism

$$
G(F((z))) \stackrel{\sim}{\rightarrow} \mathcal{G}_{F, \varpi}(F((u-\varpi)))=\mathcal{G}_{F, \varpi}(F((t))) .
$$

We denote by $s_{\mu}$ the corresponding $E^{\prime}$-valued point in $L \mathcal{G}_{F, \varpi}$.

6.a.2. Schubert varieties in mixed characteristic. We would like to define a projective scheme $M_{\mathcal{G}, \mu}$ over $\mathcal{O}_{E}$ which we might view as a generalized local model. Recall the definition of $s_{\mu} \in L \mathcal{G}_{F, \varpi}\left(E^{\prime}\right)$ and consider the $L^{+} \mathcal{G}_{F, \varpi}$-orbit $\left(L^{+} \mathcal{G}_{F, \varpi}\right)_{E^{\prime}} \cdot\left[s_{\mu}\right]$ of the corresponding point $\left[s_{\mu}\right]$ in the affine Grassmannian $\left(L \mathcal{G}_{F, \pi} / L^{+} \mathcal{G}_{F, \varpi}\right) \times_{F} E^{\prime}$. This orbit is contained in a projective subvariety of $\left(L \mathcal{G}_{F, \varpi} / L^{+} \mathcal{G}_{F, \varpi}\right) \times_{F} E^{\prime}$ which by Corollary 5.6 (1) above can be identified with the generic fiber of $\operatorname{Gr}_{\mathcal{G}, \mathcal{O}} \otimes_{\mathcal{O}} \mathcal{O}_{E^{\prime}} \rightarrow \operatorname{Spec}\left(\mathcal{O}_{E^{\prime}}\right)$. Since the conjugacy class of $\mu: \mathbb{G}_{\mathrm{m} E^{\prime}} \rightarrow G_{E^{\prime}}$ is defined over $E$, the same is true for the 
$\operatorname{orbit}\left(L^{+} \mathcal{G}_{F, \varpi}\right)_{E^{\prime}} \cdot\left[s_{\mu}\right]$ : There is an $E$-subvariety $X_{\mu}$ of $\left(L \mathcal{G}_{F, \varpi} / L^{+} \mathcal{G}_{F, \varpi}\right) \times_{F} E$ such that $X_{\mu} \times_{E} E^{\prime}=\left(L^{+} \mathcal{G}_{F, \varpi}\right)_{E^{\prime}} \cdot\left[s_{\mu}\right]$.

Definition 6.1. The generalized local model (or mixed characteristic Schubert variety) $M_{\mathcal{G}, \mu}$ is the reduced scheme over $\operatorname{Spec}\left(\mathcal{O}_{E}\right)$ which underlies the Zariski closure of the orbit $X_{\mu}$ in the ind-scheme $\operatorname{Gr}_{\mathcal{G}, \mathcal{O}_{E}}=\operatorname{Gr}_{\mathcal{G}, \mathcal{O}} \times_{\operatorname{Spec}(\mathcal{O})} \operatorname{Spec}\left(\mathcal{O}_{E}\right)$.

Since by Proposition 5.5, $\operatorname{Gr}_{\mathcal{G}, \mathcal{O}_{E}} \rightarrow \operatorname{Spec}\left(\mathcal{O}_{E}\right)$ is ind-projective, $M_{\mathcal{G}, \mu}$ is also projective over $\operatorname{Spec}\left(\mathcal{O}_{E}\right)$.

\section{6.b. Some examples.}

6.b.1. The case of $\mathrm{GL}_{N}$. Recall the notations of \$4.b.1. In particular, $\mathcal{G}$ is the group scheme over $\mathcal{O}[u]$ associated to the lattice chain $\left\{W_{i}\right\}_{i}$.

Consider the functor $\mathfrak{L}$ on $(\mathrm{Sch} / X)$ which to an $X$-scheme $y: S \rightarrow X$, associates the set of isomorphism classes of collections $\left(\mathcal{E}_{i}, \psi_{i}, \alpha_{i}\right)_{i \in \mathbb{Z}}$ where, for each $i \in \mathbb{Z}, \mathcal{E}_{i}$ are locally free coherent $\mathcal{O}_{X \times S}$-sheaves on $X \times S$ of $\operatorname{rank} N, \psi_{i}: \mathcal{E}_{i+1} \rightarrow \mathcal{E}_{i}$ are $\mathcal{O}_{X \times S}$-module homomorphisms, and $\alpha_{i}$ are $\mathcal{O}_{X \times S-\Gamma_{y}}$-module isomorphisms $\alpha_{i}: W_{i} \otimes_{\mathcal{O}[u]} \mathcal{O}_{X \times S-\Gamma_{y}} \stackrel{\sim}{\rightarrow}$ $\mathcal{E}_{i} \otimes_{\mathcal{O}_{X \times S}} \mathcal{O}_{X \times S-\Gamma_{y}}$ that satisfy the following conditions:

(a) the data are periodic of period $r,\left(\mathcal{E}_{i}, \psi_{i}, \alpha_{i}\right)=\left(\mathcal{E}_{i+r}, \psi_{i+r}, \alpha_{i+r}\right)$, for all $i \in \mathbb{Z}$,

(b) we have $\alpha_{i+1} \cdot \psi_{i}=\alpha_{i} \cdot \iota_{i}$, for all $i \in \mathbb{Z}$,

(c) Each composition of $r$ successive $\psi_{i}$ is given by multiplication by $u$, i.e

$$
\prod_{k=0}^{r-1} \psi_{i-k}=u: \mathcal{E}_{i+r}=\mathcal{E}_{i} \rightarrow \mathcal{E}_{i}
$$

for all $i \in \mathbb{Z}$, and,

(d) for each $i \in \mathbb{Z}$, the cokernel $\mathcal{E}_{i} / \psi_{i}\left(\mathcal{E}_{i+1}\right)$ is a locally free $\mathcal{O}_{S}$-module of rank $r_{i}$.

We can see that $\mathfrak{L}$ is an fpqc sheaf on $(\mathrm{Sch} / X)$.

When $S=\operatorname{Spec}(R)$ is affine, and $y: \operatorname{Spec}(R) \rightarrow X=\operatorname{Spec}(\mathcal{O}[u])$ is given by $u \mapsto y$, we have $X \times S-\Gamma_{y}=\operatorname{Spec}\left(R[u]\left[(u-y)^{-1}\right]\right)$. Since $u-y$ is not a zero divisor in $R[u]$ we can use $\alpha_{i}$ to identify $\mathcal{E}_{i}$ with the sheaf corresponding to a $R[u]$-locally free rank $N$ submodule $E_{i}$ of $R[u]\left[(u-y)^{-1}\right]^{N}$.

We can now show:

Proposition 6.2. There is a natural equivalence of functors $\operatorname{Gr}_{\mathcal{G}, X} \stackrel{\sim}{\rightarrow} \mathfrak{L}$ where $\mathcal{G}$ is the group scheme as above.

Proof. Observe that a $\mathcal{G}$-torsor $\mathcal{T}$ over $X \times S$ induces via $\mathcal{G} \hookrightarrow \prod_{i=0}^{r} \operatorname{GL}\left(W_{i}\right) \rightarrow \operatorname{GL}\left(W_{i}\right)$ a $\operatorname{GL}\left(W_{i}\right)$-torsor over $X \times S$. This amounts to giving a locally free coherent $\mathcal{O}_{X \times S}$-sheaf $\mathcal{E}_{i}$ of rank $N$; since $\mathcal{G}$ respects the maps $W_{i+1} \rightarrow W_{i}$, we obtain $\psi_{i}: \mathcal{E}_{i+1} \rightarrow \mathcal{E}_{i}$. A $\mathcal{G}$-trivialization of $\mathcal{T}$ over $X \times S-\Gamma_{y}$ produces isomorphisms $\alpha_{i}$ as above. We extend this data by periodicity; then (a), (b), (c), (d) are satisfied. This gives the arrow $\operatorname{Gr}_{\mathcal{G}, X} \rightarrow \mathfrak{L}$. To show that this is an equivalence, start with data $\left(\mathcal{E}_{i}, \psi_{i}, \alpha_{i}\right)_{i \in \mathbb{Z}}$ giving an element of $\mathfrak{L}(S)$. We would like to show that these are produced by a $\mathcal{G}$-torsor $\mathcal{T}$ with a trivialization over $X \times S-\Gamma_{y}$. It is enough to assume that $S$ is affine, $S=\operatorname{Spec}(R)$. Since, $\mathcal{G}$ is the 
subgroup of $\prod_{i \in \mathbb{Z} / r \mathbb{Z}} \mathrm{GL}\left(W_{i}\right)$ that respects $\iota_{i}$, we can now see that it is enough to show the following: Locally for the Zariski topology on $R$, there are isomorphisms

$$
\lambda_{i}: W_{i} \otimes R[u] \stackrel{\sim}{\rightarrow} \mathcal{E}_{i}
$$

such that $\lambda_{i} \cdot \iota_{i}=\psi_{i} \cdot \lambda_{i+1}$. This follows by an argument similar to the proof of [55, Appendix to Ch. 3, Prop. A.4].

Now if $\mu: \mathbb{G}_{\mathrm{m}} \rightarrow \mathrm{GL}_{N}$ is the minuscule coweight given by $a \mapsto \operatorname{diag}\left(a^{(d)}, 1^{(N-d)}\right)$ and $\mathcal{O}=W\left(\mathbb{F}_{p}\right)$, we can see, using Proposition 6.2, that in this situation, the local models $M_{\mathcal{G}, \mu}$ agree with the Rapoport-Zink local models for $\mathrm{GL}_{N}$ and $\mu$ considered in [55. Indeed, in this case, by [19], the local models of [55] are flat over $\mathcal{O}$ and so they agree with the $M_{\mathcal{G}, \mu}$ above.

6.b.2. The case of $\mathrm{GSp}_{2 n}$. Recall the notations of 4 .b.2. In particular, $\mathcal{G}$ is the group scheme over $\mathcal{O}[u]$ associated to the self-dual lattice chain $\left\{W_{i}\right\}_{i}$.

Consider the functor $\mathfrak{L} \mathfrak{S P}$ on $(\mathrm{Sch} / X)$ which to an $X$-scheme $y: S \rightarrow X$, associates the set of isomorphism classes of collections $\left(\mathcal{E}_{i}, \psi_{i}, \alpha_{i}, h_{i}\right)_{i \in \mathbb{Z}}$ where $\left(\mathcal{E}_{i}, \psi_{i}, \alpha_{i}\right)_{i \in \mathbb{Z}}$ give an object of $\mathfrak{L}$ and in addition

$$
h_{i}: \mathcal{E}_{i} \times \mathcal{E}_{-i-a} \rightarrow \mathcal{O}_{X \times S}
$$

are perfect $\mathcal{O}_{X \times S}$-bilinear forms that satisfy

a) $h_{i}\left(\psi_{i}(x), y\right)=h_{i+1}\left(x, \psi_{-i-1-a}(y)\right)$, for $x \in \mathcal{E}_{i+1}, y \in \mathcal{E}_{-i-a}$.

b) There is $c \in \mathcal{O}_{X \times S-\Gamma_{y}}^{*}$, such that $h_{i} \cdot\left(\alpha_{i}, \alpha_{-i-a}\right)=c \cdot h$ for all $i \in \mathbb{Z}$ (as forms $\left.W_{i} \otimes \mathcal{O}_{X \times S-\Gamma_{y}} \times W_{-i-a} \otimes \mathcal{O}_{X \times S-\Gamma_{y}} \rightarrow \mathcal{O}_{X \times S-\Gamma_{y}}\right)$.

A similar argument as above, (cf. [55, Appendix to Ch. 3, Prop. A. 21]) now gives

Proposition 6.3. There is a natural equivalence of functors $\operatorname{Gr}_{\mathcal{G}, X} \stackrel{\sim}{\rightarrow} \mathfrak{L} \mathfrak{S P}$ where $\mathcal{G}$ is the (symplectic) group scheme as above.

Again as a result of the above, combined with the flatness result of [20], we can see that if $\mu: \mathbb{G}_{\mathrm{m}} \rightarrow \mathrm{GSp}_{2 n}$ is the standard minuscule coweight given by $a \mapsto \operatorname{diag}\left(a^{(n)}, 1^{(n)}\right)$ and $\mathcal{O}=W\left(\mathbb{F}_{p}\right)$, then the local models $M_{\mathcal{G}, \mu}$ in this situation agree with the local models for $\mathrm{GSp}_{2 n}$ considered in [55].

6.b.3. One can find a similar interpretation of $\mathrm{Gr}_{\mathcal{G}, X}$ as moduli spaces of chains of bundles with additional structure given by suitable forms in more cases as in $4 . \mathrm{4}$, for example when $G$ is an orthogonal group or a (ramified) unitary group. We will leave the details to the reader. A corresponding statement comparing the local models $M_{\mathcal{G}, \mu}$ with the local models in the theory of PEL Shimura varieties ([55], 20], [19], 447, [48], [50], [51]) will be explained in the next paragraph. 


\section{Shimura VARIETIES AND LOCAL MOdels}

Here we discuss Shimura varieties and their integral models over primes where the level subgroup is parahoric. We conjecture that there exist integral models that fit in a "local model diagram" in which the local model is given by our construction in the previous chapter. We show this in most cases of Shimura varieties of PEL type. We also explain how Theorem 0.2 of the introduction follows from our main structural results on local models (which will be shown in the next section).

7.a. The local model diagram. Let $S h_{\mathbf{K}}=S h(\mathbf{G},\{h\}, \mathbf{K})$ denote a Shimura variety [14] attached to the triple consisting of a connected reductive group $\mathbf{G}$ over $\mathbb{Q}$, a family of Hodge structures $h$ and a compact open subgroup $\mathbf{K} \subset \mathbf{G}\left(\mathbb{A}_{f}\right)$. We fix a prime number $p$ and assume that $\mathbf{K}$ factorizes as $\mathbf{K}=K^{p} \cdot K_{p} \subset \mathbf{G}\left(\mathbb{A}_{f}^{p}\right) \times \mathbf{G}\left(\mathbb{Q}_{p}\right)$. We assume in addition that $K=K_{p}$ is a parahoric subgroup of $\mathbf{G}\left(\mathbb{Q}_{p}\right)$, i.e it corresponds to the connected stabilizer of a point of the Bruhat-Tits building of $\mathbf{G} \otimes_{\mathbb{Q}} \mathbb{Q}_{p}$. We denote by $\mathcal{P}$ the corresponding Bruhat-Tits group scheme over $\mathbb{Z}_{p}$.

Let $\mathbf{E} \subset \mathbb{C}$ denote the reflex field of $(\mathbf{G},\{h\})$, i.e. the field of definition of the geometric conjugacy class of one-parameter subgroups $\{\mu\}=\left\{\mu_{h}\right\}$ attached to $\{h\}$, cf. [14. Then $\mathbf{E}$ is a finite extension of $\mathbb{Q}$. Fixing an embedding $\overline{\mathbb{Q}} \rightarrow \overline{\mathbb{Q}}_{p}$ determines a place $\wp$ of $\mathbf{E}$ above $p$. We denote by the same symbol the canonical model of $S h_{\mathbf{K}}$ over $\mathbb{E}$ and its base change to $\mathbb{E}_{\wp}$. For simplicity, set $E=\mathbf{E}_{\wp}$ and denote by $\mathcal{O}_{E}$ the ring of integers of $E$ and by $k_{E}$ its residue field. It is then an interesting problem to define a suitable model $\mathcal{S}_{\mathbf{K}}$ of $S h_{\mathbf{K}}$ over $\operatorname{Spec}\left(\mathcal{O}_{E}\right)$. Such a model should be projective if $S h_{\mathbf{K}}$ is (which is the case when $\mathbf{G}_{\text {ad }}$ is $\mathbb{Q}$-anisotropic), and should always have manageable singularities. In particular, it should be flat over $\operatorname{Spec}\left(\mathcal{O}_{E}\right)$, and its local structure should only depend on the "localized" group $G=\mathbf{G} \otimes \mathbb{Q} \mathbb{Q}_{p}$, the geometric conjugacy class $\{\mu\}$ over $\overline{\mathbb{Q}}_{p}$, and the parahoric subgroup $K=K_{p}$ of $G\left(\mathbb{Q}_{p}\right)$. Note that, due to the definition of a Shimura variety, the conjugacy class $\{\mu\}$ is minuscule.

Suppose now in addition that the group $G$ splits over a tamely ramified extension of $\mathbb{Q}_{p}$. We can then apply the constructions of the previous paragraphs to $G, \mathcal{O}=\mathbb{Z}_{p}$, and a point of the building $\mathcal{B}\left(G, \mathbb{Q}_{p}\right)$ that corresponds to $K \subset G\left(\mathbb{Q}_{p}\right)$. By Theorem 3.1, we obtain a smooth affine group scheme $\mathcal{G} \rightarrow \operatorname{Spec}\left(\mathbb{Z}_{p}[u]\right)$; the choice of $\{\mu\}$ allows us to give a projective scheme $M_{\mathcal{G}, \mu} \rightarrow \operatorname{Spec}\left(\mathcal{O}_{E}\right)$. Let us set

$$
\mathrm{M}(G,\{\mu\})_{K}=M_{\mathcal{G}, \mu} .
$$

By its construction, $\mathrm{M}(G,\{\mu\})_{K}$ affords an action of the group scheme $\mathcal{G} \otimes_{\mathbb{Z}_{p}[u], u \mapsto p} \mathcal{O}_{E}=$ $\mathcal{P} \otimes_{\mathbb{Z}_{p}} \mathcal{O}_{E}$. The conjecture is that there exists a model $\mathcal{S}_{\mathbf{K}}$ of the Shimura variety over $\mathcal{O}_{E}$ whose singularities are "described by the local model $\mathrm{M}(G,\{\mu\})_{K}$ ". More precisely:

We conjecture that there is such a $\mathcal{S}_{\mathbf{K}}$ that affords a local model diagram

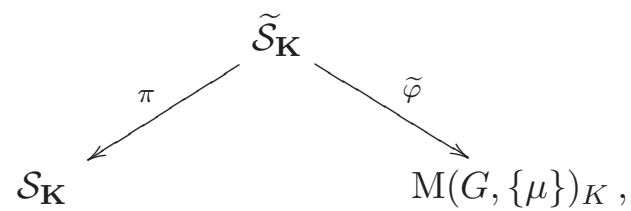


of $\mathcal{O}_{E}$-schemes, in which:

- $\pi$ is a torsor under the group $\mathcal{P}_{\mathcal{O}_{E}}=\mathcal{P} \otimes_{\mathbb{Z}_{p}} \mathcal{O}_{E}$,

- $\widetilde{\varphi}$ is $\mathcal{P}_{\mathcal{O}_{E}}$-equivariant and smooth of relative dimension $\operatorname{dim} G$.

(Equivalently, using the language of algebraic stacks, there should be a smooth morphism of algebraic stacks

$$
\varphi: \mathcal{S}_{\mathbf{K}} \rightarrow\left[\mathrm{M}(G,\{\mu\})_{K} / \mathcal{P}_{\mathcal{O}_{E}}\right]
$$

of relative dimension $\operatorname{dim} G$ where in the brackets we have the stack quotient. See also [51].) In fact, we conjecture that there is such a diagram with $\widetilde{\varphi}$ being in addition surjective.

The existence of the local model diagram implies the following: Suppose $x$ is a point of $\mathcal{S}_{\mathbf{K}}$ with values in the finite field $\mathbb{F}_{q}$. By Lang's theorem the $\mathcal{P}_{\mathcal{O}_{E}}$-torsor $\pi$ splits over $x$, and so there is $\tilde{x} \in \widetilde{\mathcal{S}}_{\mathbf{K}}\left(\mathbb{F}_{q}\right)$ with image $y=\tilde{\phi}(\tilde{x}) \in \mathrm{M}(G,\{\mu\})_{K}\left(\mathbb{F}_{q}\right)$ such that the henselizations of $\mathcal{S}_{\mathbf{K}}$ at $x$ and of $\mathrm{M}(G,\{\mu\})_{K}$ at $y$ are isomorphic. The $\mathcal{P}_{\mathcal{O}_{E}}$-orbit of $y$ in $\mathrm{M}(G,\{\mu\})_{K}$ is well-defined.

7.a.1. Now suppose that there is a closed group scheme immersion $\rho: \mathbf{G} \hookrightarrow \operatorname{GSp}_{2 n}$ such that the composition of $\rho$ with $\mu$ is in the conjugacy class of the standard minuscule cocharacter of $\mathrm{GSp}_{2 n}$. For typesetting simplicity, set $k=\overline{\mathbb{F}}_{p}=\bar{k}_{E}$. We will also assume that there is a self-dual "lattice" chain $W_{\bullet}=\left\{W_{i}\right\}_{i \in \mathbb{Z}}$ in $\mathbb{Z}_{p}[u]^{2 n}$ as in 6 .b.2 such that

- the homomorphism $\mathbf{G} \rightarrow \mathrm{GSp}_{2 n}$ extends to a homomorphism $\mathcal{G} \rightarrow \operatorname{GSp}\left(W_{\bullet}\right)$,

- the homomorphism $\mathcal{G} \otimes_{\mathbb{Z}_{p}[u]} k[[u]] \rightarrow \operatorname{GSp}\left(W_{\bullet}\right) \otimes_{\mathbb{Z}_{p}[u]} k[[u]]$ is a locally closed immersion, the Zariski closure of $\mathcal{G} \otimes_{\mathbb{Z}_{p}[u]} k((u))$ in $\operatorname{GSp}\left(W_{\bullet}\right) \otimes_{\mathbb{Z}_{p}[u]} k[[u]]$ is a smooth group scheme $P_{k}^{\prime}$ over $k[[u]]$ and $P_{k}^{\prime}(k[[u]])$ stabilizes $x_{k((u))}$ in the building of $\mathcal{G}(k((u)))$; then $P_{k}:=\mathcal{G} \otimes_{\mathbb{Z}_{p}[u]} k[[u]]$ is the neutral component of $P_{k}^{\prime}$.

Under these assumptions, extending torsors via the homomorphism $\mathcal{G} \rightarrow \operatorname{GSp}\left(W_{\bullet}\right)$ gives $\operatorname{Gr}_{\mathcal{G}, \mathbb{Z}_{p}} \rightarrow \operatorname{Gr}_{\mathrm{GSp}\left(W_{\bullet}\right), \mathbb{Z}_{p}}$. Restricting to $\mathrm{M}(G,\{\mu\})_{K} \hookrightarrow \mathrm{Gr}_{\mathcal{G}, \mathbb{Z}_{p}} \otimes_{\mathcal{O}} \mathcal{O}_{E}$ gives a morphism of schemes

$$
\iota: \mathrm{M}(G,\{\mu\})_{K} \rightarrow \mathrm{M}\left(\mathrm{GSp}_{2 n}\right)_{W_{\bullet}} \otimes_{\mathbb{Z}_{p}} \mathcal{O}_{E}
$$

where $\mathrm{M}\left(\mathrm{GSp}_{2 n}\right)_{W_{\bullet}}$ is the symplectic local model as in [20] (cf. 6.b.2).

Proposition 7.1. Under the above assumptions, $\iota: \mathrm{M}(G,\{\mu\})_{K} \rightarrow \mathrm{M}\left(\mathrm{GSp}_{2 n}\right)_{W_{\bullet}} \otimes_{\mathbb{Z}_{p}} \mathcal{O}_{E}$ is a closed immersion.

Proof. Recall that the generic fiber $\mathrm{M}(G,\{\mu\})_{K} \otimes_{\mathcal{O}_{E}} E$ of $\mathrm{M}(G,\{\mu\})_{K}$ is the flag variety of parabolics corresponding to $\{\mu\}$; the generic fiber of $\mathrm{M}\left(\mathrm{GSp}_{2 n}\right)_{W_{\bullet}}$ is the Lagrangian Grassmannian $\operatorname{LGr}(n, 2 n)$ of $n$-dimensional isotropic subspaces; our assumption on $\{\rho \circ \mu\}$ implies that $\iota \otimes_{\mathcal{O}_{E}} E$ is a closed immersion. We will now explain why, in this set-up, the morphism on the special fibers $\iota \otimes_{\mathcal{O}_{E}} k_{E}$ is also a closed immersion.

As above $P_{k}=\mathcal{G} \otimes_{\mathbb{Z}_{p}[u]} k[[u]]$ and $P_{k}^{\prime}$ is the closure of $P_{k}\left[u^{-1}\right]$ in $\operatorname{GSp}\left(W_{\bullet}\right) \otimes_{\mathbb{Z}_{p}[u]} k[[u]]$; $P_{k}$ is a parahoric group scheme over $k[[u]]$. By our assumption, $P_{k}^{\prime}$ is a smooth affine group scheme over $k[[u]]$ and $P_{k}$ is the neutral component of $P_{k}^{\prime}$. Both $\operatorname{Gr}_{P_{k}}$ and $\operatorname{Gr}_{P_{k}^{\prime}}$ 
are ind-proper ind-schemes over $k$ and the natural morphism

$$
\mathrm{Gr}_{\mathcal{G}, k}=\operatorname{Gr}_{P_{k}} \rightarrow \operatorname{Gr}_{P_{k}^{\prime}}
$$

is finite étale. In what follows, for simplicity, set $P=P_{k}, P^{\prime}=P_{k}^{\prime}$. Consider the Kottwitz homomorphism $\kappa: P^{\prime}(k((u)))=P(k((u))) \rightarrow \pi_{1}\left(P\left[u^{-1}\right]\right)_{I}$ for the reductive group $P\left[u^{-1}\right]=P^{\prime}\left[u^{-1}\right]$ over $k((u))$. By [26], since $P^{\prime}(k[[u]])$ stabilizes $x_{k((u))}$, the intersection of the kernel $\operatorname{ker}(\kappa)$ with $P^{\prime}(k[[u]])$ is equal to $P(k[[u]])$. By [49], the homomorphism $\kappa$ induces a bijection

$$
\pi_{0}(L P) \simeq \pi_{0}\left(\mathrm{Gr}_{P}\right) \stackrel{\sim}{\rightarrow} \pi_{1}\left(P\left[u^{-1}\right]\right)_{I}
$$

between the set of connected components of $\operatorname{Gr}_{P}$ and the group $\pi_{1}\left(P\left[u^{-1}\right]\right)_{I}$. The above now imply that $\operatorname{Gr}_{\mathcal{G}, k}=\operatorname{Gr}_{P} \rightarrow \operatorname{Gr}_{P^{\prime}}$ identifies each connected component of $\operatorname{Gr}_{P}=\operatorname{Gr}_{\mathcal{G}, k}$ with a connected component of $\operatorname{Gr}_{P^{\prime}}$.

Now $P^{\prime}$ is a closed subgroup scheme of $Q:=\operatorname{GSp}\left(W_{\bullet}\right) \otimes_{\mathcal{O}[u]} k[[u]]$. By [1] and [56, VI. $2.5]$, the quotient $Q / P^{\prime}$ is quasi-projective over $k[[u]]$. Suppose that $A$ is an Artin local $k$ algebra. Then $A((u))$ is a local ring and so each morphism $\operatorname{Spec}(A((u))) \rightarrow Q / P^{\prime}$ factors through an open affine subscheme of $Q / P^{\prime}$. Using this together with the argument of [17, Appendix], we can see that the fibered product $\operatorname{Spec}(A) \times{ }_{\mathrm{Gr}_{Q}} \operatorname{Gr}_{P^{\prime}}$ is represented by the closed subscheme of $\operatorname{Spec}(A)$ where the morphism $\operatorname{Spec}\left(A^{\prime}((u))\right) \rightarrow Q / P^{\prime}$ obtained from a corresponding $\operatorname{Spec}(A((u))) \rightarrow Q / P^{\prime}$ extends to $\operatorname{Spec}\left(A^{\prime}[[u]]\right) \rightarrow Q / P^{\prime}$. In particular, for any such $A, \operatorname{Gr}_{P^{\prime}}(A) \rightarrow \operatorname{Gr}_{Q}(A)$ is injective. Now let $\operatorname{Gr}_{P^{\prime}}=\underline{\lim }_{i} Y_{i}, \operatorname{Gr}_{Q}=\underline{\lim }_{j} Z_{j}$, with $Y_{i}, Z_{j}$ proper closed subschemes and suppose $Y_{i}$ maps to $Z_{j(i)}$. Applying the above, we see that $f_{i}: Y_{i} \rightarrow Z_{j(i)}$ is quasi-finite; since $Y_{i}$ is proper, $f_{i}$ is also proper and hence finite by Zariski's main theorem. Since $f_{i}(A)$ is injective for all $A$ as above, we see that $f_{i}$ is a closed immersion. We conclude that

$$
\mathrm{Gr}_{P^{\prime}} \rightarrow \mathrm{Gr}_{Q}
$$

is a closed immersion. Now notice that Zariski's main theorem implies that the special fiber $\mathrm{M}(G,\{\mu\})_{K} \otimes_{\mathcal{O}_{E}} k$ of $\mathrm{M}(G,\{\mu\})_{K}$ is connected: indeed, the generic fiber $\mathrm{M}(G,\{\mu\})_{K} \otimes_{\mathcal{O}_{E}} E$ over $E$ is geometrically connected and $\mathrm{M}(G,\{\mu\})_{K} \rightarrow \operatorname{Spec}\left(\mathcal{O}_{E}\right)$ is proper by construction. Since each connected component of $\operatorname{Gr}_{\mathcal{G}, k}=\operatorname{Gr}_{P}$ identifies with a connected component of $\operatorname{Gr}_{P^{\prime}}$ and $\mathrm{M}(G,\{\mu\})_{K} \otimes_{\mathcal{O}_{E}} k$ is connected we conclude from by above that the morphism $\mathrm{M}(G,\{\mu\})_{K} \otimes_{\mathcal{O}_{E}} k \rightarrow \mathrm{Gr}_{Q}$ is a closed immersion. Therefore the morphism $\iota \otimes_{\mathcal{O}_{E}} k$ is also a closed immersion.

We will now show that $\iota$ is a closed immersion. For simplicity, set $M=M(G,\{\mu\})_{K}$. Denote by $\iota(\mathrm{M})$ the closed scheme theoretic image of $\iota: \mathrm{M} \rightarrow \mathrm{M}\left(\mathrm{GSp}_{2 n}\right)_{W_{\bullet}} \otimes_{\mathcal{O}} \mathcal{O}_{E}$. (Since $\iota \otimes \mathcal{O}_{E} E$ is a closed immersion and $\mathrm{M}$ is integral, $\iota(M)$ coincides with the Zariski closure of $\mathrm{M} \otimes_{\mathcal{O}_{E}} E$ in $\left.\mathrm{M}\left(\mathrm{GSp}_{2 n}\right)_{W_{\bullet}} \otimes_{\mathcal{O}} \mathcal{O}_{E}.\right)$ We would like to show that $\mathrm{M}=\iota(\mathrm{M})$. Once again, by Zariski's main theorem $\iota(\mathrm{M})_{k}=\iota(\mathrm{M}) \otimes_{\mathcal{O}_{E}} k$ is connected. Using the valuative criterion of properness and the fact that both $\mathrm{M}$ and $\iota(\mathrm{M})$ are proper and flat over $\mathcal{O}_{E}$, we see that $\mathrm{M}_{k} \rightarrow \iota(\mathrm{M})_{k}$ is surjective. Consider $\iota \otimes_{\mathcal{O}_{E}} k: \mathrm{M}_{k} \rightarrow \iota(\mathrm{M})_{k} \hookrightarrow \mathrm{M}\left(\mathrm{GSp}_{2 n}\right)_{W} \otimes_{\mathcal{O}} k$; this is a closed immersion, and therefore so is $\mathrm{M}_{k} \rightarrow \iota(\mathrm{M})_{k}$. The map $\mathrm{M} \rightarrow \iota(\mathrm{M})$ is proper and quasi-finite, hence finite. Let $A$ be the local ring of $\iota(\mathrm{M})$ at a closed point $\iota(x)$ of $\iota(\mathrm{M})_{k}$ which is the image of a closed point $x$ of $\mathrm{M}_{k}$. Denote by $B$ the local ring of $\mathrm{M}$ at $x$, then 
$A \subset B$. Also since $x$ is the unique point of $\mathrm{M}$ that maps to $\iota(x), B$ is finitely generated over $A$. Since $\iota \otimes_{\mathcal{O}_{E}} k$ is a closed immersion, $A / \varpi_{E} A$ surjects onto $B / \varpi_{E} B$ and hence $\varpi_{E} \cdot B / A=(0)$. Applying Nakayama's lemma to the finitely generated $A$-module $B / A$ we can conclude $A=B$. From this and the above we deduce $\mathrm{M}=\iota(\mathrm{M})$.

7.b. The PEL case. In this paragraph, we elaborate on the local models for Shimura varieties of PEL type. We will assume throughout that the prime $p$ is odd.

We follow [55, Chapter 6] (see also [36]): Let $\mathbf{B}$ be a finite dimensional semisimple algebra over $\mathbb{Q}$ with a positive involution $*$. Then the center $\mathbf{F}$ of $\mathbf{B}$ is a product of $\mathbf{C M}$ fields and totally real fields. Let $\mathbf{V}$ be a finite dimensional $\mathbb{Q}$-vector space of dimension $2 n$ with a perfect alternating $\mathbb{Q}$-bilinear form $():, \mathbf{V} \times \mathbf{V} \rightarrow \mathbb{Q}$. Assume that $\mathbf{V}$ is equipped with a B-module structure, such that

$$
(b v, w)=\left(y, b^{*} w\right), \quad \forall v, w \in \mathbf{V}, \quad b \in \mathbf{B} .
$$

Set $\mathbf{G} \subset \operatorname{Aut}_{\mathbf{B}}(\mathbf{V})$ to be the closed algebraic subgroup over $\mathbb{Q}$ such that

$$
\mathbf{G}(\mathbb{Q})=\left\{g \in \operatorname{Aut}_{\mathbf{B}}(\mathbf{V}) \mid(g v, g w)=c(g)(v, w), \forall v, w \in \mathbf{V}, c(g) \in \mathbb{Q}\right\} .
$$

Let $h: \mathbf{S}:=\operatorname{Res} \mathbb{C}_{\mathbb{R}} \mathbb{G}_{\mathrm{m} \mathbb{C}} \rightarrow \mathbf{G}_{\mathbb{R}}$ be a morphism that defines on $\mathbf{V}_{\mathbb{R}}$ a Hodge structure of type $(1,0),(0,1)$, such that $(v, h(\sqrt{-1}) w)$ is a symmetric positive bilinear form on $\mathbf{V}_{\mathbb{R}}$. This gives a B-invariant decomposition $\mathbf{V}_{\mathbb{C}}=\mathbf{V}_{0, \mathbb{C}} \oplus \mathbf{V}_{1, \mathbb{C}}$ where $z \in \mathbf{S}$ acts on $\mathbf{V}_{0, \mathbb{C}}$ by multiplication by $\bar{z}$ and on $\mathbf{V}_{1, \mathbb{C}}$ by multiplication by $z$. Then $(\mathbf{G},\{h\})$ defines a Shimura variety of PEL type (cf. [14, [36]) 5 The reflex field $\mathbf{E}$ is the subfield of $\mathbb{C}$ that is generated by the traces $\operatorname{Tr}_{\mathbb{C}}\left(b \mid \mathbf{V}_{0, \mathbb{C}}\right)$ for $b \in \mathbf{B}$. Using the isomorphism $\mathbf{S}_{\mathbb{C}} \simeq \mathbb{C}^{*} \times \mathbb{C}^{*}, z \rightarrow(z, \bar{z})$, we define $\mu: \mathbb{G}_{\mathrm{m} \mathbb{C}} \rightarrow \mathbf{G}_{\mathbb{C}}$ as $\mu(z)=h_{\mathbb{C}}(z, 1)$; the field $\mathbf{E}$ is the field of definition of the G-conjugacy class of $\mu$. By definition, we have an embedding $\rho: \mathbf{G} \hookrightarrow \operatorname{GSp}(\mathbf{V},())=,\operatorname{GSp}_{2 n}$ and $\rho \circ \mu$ is conjugate to the standard minuscule coweight of $\mathrm{GSp}_{2 n}$.

7.b.1. Now set $G^{b}=\mathbf{G}_{\mathbb{Q}_{p}}$ and denote by $G=\mathbf{G}_{\mathbb{Q}_{p}}^{\circ}$ the neutral component. Let $\mathfrak{P} \mid(p)$ be a prime of $\mathbf{E}$ and set $E^{b}=\mathbf{E}_{\mathfrak{P}} \subset \overline{\mathbb{Q}}_{p}$; we see that $\mu$ gives a unique conjugacy class of coweights $\mathbb{G}_{\mathrm{m} \overline{\mathbb{Q}}_{p}} \rightarrow G_{\overline{\mathbb{Q}}_{p}}^{b}$ which is defined over $E^{b}$. Observe that each cocharacter $\mu: \mathbb{G}_{\mathrm{m} \overline{\mathbb{Q}}_{p}} \rightarrow G_{\overline{\mathbb{Q}}_{p}}^{b}$ lands in the neutral component $G_{\overline{\mathbb{Q}}_{p}}$ and we can choose a representative $\mu: \mathbb{G}_{\mathrm{m} \overline{\mathbb{Q}}_{p}} \rightarrow G_{\overline{\mathbb{Q}}_{p}}$. Then the corresponding geometric $G_{\overline{\mathbb{Q}}_{p}}$-conjugacy class is defined over $E$ which is a finite extension of $E^{b}$ (and can depend on our choice). We will also denote this conjugacy class by $\{\mu\}$. As in [55] we suppose that there exists an order $\mathcal{O}_{\mathbf{B}}$ of $B$ such that $\mathcal{O}_{B}:=\mathcal{O}_{\mathbf{B}} \otimes \mathbb{Z}_{p}$ is a maximal order which is stable under the involution $*$. Let $\{\mathcal{L}\}$ be a self-dual multi-chain of $\mathcal{O}_{B}$-lattices in $V=\mathbf{V}_{\mathbb{Q}_{p}}$ with respect to $*$ and the alternating form $\left(\right.$, ) (in the sense of [55, Chapter 3]). Consider the group scheme $\mathcal{P}^{b}$ over $\mathbb{Z}_{p}$ whose $S$-valued points is the group of $\mathcal{O}_{B} \otimes \mathcal{O}_{S}$-isomorphisms of the multi-chain $\left\{\mathcal{L} \otimes \mathcal{O}_{S}\right\}$ that respect the forms up to (common) similitude in $\mathcal{O}_{S}^{*}$. The generic fiber of $\mathcal{P}^{b}$ is $G^{b}$. It follows from [55, Appendix to Ch. 3] that $\mathcal{P}^{b}$ is smooth over $\mathbb{Z}_{p}$.

\footnotetext{
${ }^{5}$ Note that $\mathbf{G}$ is not always connected and so the set-up differs slightly from the previous paragraph where it was assumed that $\mathbf{G}$ is connected.
} 
As we discussed in 4.a, each such self-dual multichain $\{\mathcal{L}\}$ gives a point $x(\mathcal{L})$ in the Bruhat-Tits building $\mathcal{B}\left(G, \breve{\mathbb{Q}}_{p}\right)$; the group $\mathcal{P}^{b}\left(\breve{\mathbb{Z}}_{p}\right) \cap G\left(\breve{\mathbb{Q}}_{p}\right)$ is the stabilizer subgroup of $x(\mathcal{L})$ in $G\left(\breve{\mathbb{Q}}_{p}\right)$. By [7], there is a unique affine smooth group scheme $\mathcal{P}^{\prime}$ over $\operatorname{Spec}\left(\mathbb{Z}_{p}\right)$ with generic fiber $G$ such that $\mathcal{P}^{\prime}\left(\breve{\mathbb{Z}}_{p}\right)$ is the stabilizer of $x(\mathcal{L})$ in $G\left(\breve{\mathbb{Q}}_{p}\right)$. Then there is a group scheme embedding $\mathcal{P}^{\prime} \hookrightarrow \mathcal{P}^{b}$ which extends $G=\left(G^{b}\right)^{\circ} \hookrightarrow G^{b}$ and in fact, $\mathcal{P}^{\prime}$ is the Zariski closure of $G$ in $\mathcal{P}^{b}$. Finally, the neutral component $\mathcal{P}:=\left(\mathcal{P}^{\prime}\right)^{\circ}$ is the parahoric group scheme of $G$ associated to $x(\mathcal{L})$.

7.b.2. In this paragraph we describe some constructions from [55]. The reader is referred to this work for more details.

We will consider $B:=\mathbf{B} \otimes_{\mathbb{Q}} \mathbb{Q}_{p}$ as a central $F:=\mathbf{F} \otimes_{\mathbb{Q}} \mathbb{Q}_{p}$-algebra. For simplicity, we will assume that the invariants of the involution $*$ on the center $F$ of $B$ are a field $F_{0}$. (The general case of $(B, *, V,()$,$) can be decomposed into a direct sum of cases with$ this property.) There are two cases:

(A) The center of $B$ is a product $Z=F \times F$, then $B \simeq M_{n}(D) \times M_{n}\left(D^{\text {opp }}\right)$ and $(x, y)^{*}=(y, x)$. (Here $D$ is a central division algebra over $F$. Note that $D=D^{\text {opp }}$ as sets.)

(B) The center of $B \simeq M_{n}(D)$ is a field $F$.

(Case A) We set $\mathcal{O}_{B}=M_{n}\left(\mathcal{O}_{D}\right) \times M_{n}\left(\mathcal{O}_{D}{ }^{\text {opp }}\right)$; denote by $*$ the exchange involution on $\mathcal{O}_{B}$. Set $U=\mathcal{O}_{D}^{n} \otimes_{\mathcal{O}_{D}} T$ (a left $M_{n}\left(\mathcal{O}_{D}\right)$-module) where $T \simeq \mathcal{O}_{D}^{m}$ and $\tilde{U}=\operatorname{Hom}_{\mathbb{Z}_{p}}\left(U, \mathbb{Z}_{p}\right)$ which is then also naturally a left $M_{n}\left(\mathcal{O}_{D}{ }^{\text {opp }}\right)$-module. Set $W=U \oplus \widetilde{U}$ which is a left $\mathcal{O}_{B}$-module; then $W$ also supports a unique perfect alternating $\mathbb{Z}_{p}$-bilinear form ( , ) for which $U, \widetilde{U}$ are isotropic and $((u, 0),(0, \tilde{u}))=\tilde{u}(u)$ for all $u \in U, \tilde{u} \in \widetilde{U}$. In this case, the lattice chain $\mathcal{L}_{\bullet}$ comes about as follows: Choose an $\mathcal{O}_{D}$-lattice chain $\Gamma_{\bullet}$ in $\mathcal{O}_{D}^{m}=\oplus_{i=1}^{m} \mathcal{O}_{D} e_{i}$

$$
\Gamma_{r}=\Pi \cdot \Gamma_{0} \subset \Gamma_{r-1} \subset \cdots \subset \Gamma_{0}=\mathcal{O}_{D}^{m}
$$

such that $\Gamma_{j}=\oplus_{j=1}^{m} \Pi^{a_{j}} \mathcal{O}_{D}$ for some $1 \geq a_{j} \geq 0$. Consider

$$
U_{\bullet}=\mathcal{O}_{D}^{n} \otimes_{\mathcal{O}_{D}} \Gamma_{\bullet}
$$

Then $\mathcal{L}_{\bullet}=U_{\bullet} \oplus \widetilde{U_{\bullet}}$ for a unique choice of $\Gamma_{\bullet}$ as above.

(Case B) This can be split into three cases (B1), (B2), (B3) which correspond to (II), (III), (IV) of [55], p. 135.

(B1) $B=M_{n}(F), F=F_{0}$.

(B2) $B=M_{n}(F)$ and $F / F_{0}$ is a quadratic extension.

(B3) $B=M_{n}(D)$ where $D$ is a quaternion algebra over $F$ and $F=F_{0}$.

Recall that, as in [55], we always assume that we have a maximal order $\mathcal{O}_{D}$ such that $\mathcal{O}_{B}=M_{n}\left(\mathcal{O}_{D}\right)$ is stable under the involution $*$. Here, we use $D$ to denote either the quaternion algebra $D$ or $F$ depending on the case we are considering. As in [55, Appendix to Ch. 3], we see that there is a certain perfect form $H: \mathcal{O}_{D}^{n} \times \mathcal{O}_{D}^{n} \rightarrow \mathcal{O}_{D}$ (the possible types are: symmetric, alternating, hermitian, anti-hermitian, quaternionic hermitian or antihermitian for the main or the new involution on $D$ ) on the (right) $\mathcal{O}_{D}$-module $U=\mathcal{O}_{D}^{n}$ 
such that the involution $*$ satisfies

$$
H(A u, v)=H\left(u, A^{*} v\right), \quad u, v \in U, A \in M_{n}\left(\mathcal{O}_{D}\right) .
$$

Here we identify $M_{n}\left(\mathcal{O}_{D}\right)$ with the right- $\mathcal{O}_{D}$-module endomorphisms End $\mathcal{O}_{D}(U)$ of $U$. We will denote the involution of $\mathcal{O}_{D}$ that we are using by $d \mapsto \breve{d}$. Then $d \mapsto \breve{d}$ can be trivial, conjugation, the main involution or the new involution in the case of quaternion algebras. For simplicity, we will also refer to all the possible types of forms as $\epsilon$-hermitian for the involution $d \mapsto \breve{d}$. If this involution is trivial, "1-hermitian" means symmetric and "(-1)-hermitian" means alternating.

Let $\vartheta_{F}$ be a generator of the different of $F / \mathbb{Q}$ such that $\bar{\vartheta}_{F}=-\vartheta_{F}$ if $F \neq F_{0}$. Define $h: \mathcal{O}_{D} \times \mathcal{O}_{D} \rightarrow \mathbb{Z}_{p}$ by $h=\operatorname{Tr}_{F / \mathbb{Q}_{p}}\left(\vartheta_{F}^{-1} H\right)$ if $D=F$, and by $h=\operatorname{Tr}_{F / \mathbb{Q}_{p}}\left(\vartheta_{F}^{-1} \operatorname{Tr}^{0}\left(\Pi^{-1} H\right)\right)$ if $D$ is quaternion, where $\operatorname{Tr}^{0}: D \rightarrow F$ is the reduced trace.

As explained in [55, Appendix to Ch. 3], we can now employ Morita equivalence and write $V=M_{n}(D) \otimes_{D} W$ with $W \simeq D^{m}$ a free left $D$-module. The alternating form $()=,\mathcal{E}($,$) on V$ can be written

$$
\mathcal{E}\left(u_{1} \otimes w_{1}, u_{2} \otimes w_{2}\right)=h\left(u_{1}, u_{2} \Psi\left(w_{1}, w_{2}\right)\right)
$$

where $\Psi: W \times W \rightarrow D$ is an $\epsilon$-hermitian form on $W$. The sign $\epsilon$ of $\Psi$ is the opposite of that of $H$. We can also write

$$
\mathcal{L}_{i}=M_{n}\left(\mathcal{O}_{D}\right) \otimes_{\mathcal{O}_{D}} N_{i}
$$

where $N_{i}$ are $\mathcal{O}_{D}$-lattices in $W$. The perfect forms $\mathcal{E}: \mathcal{L}_{i} \times \mathcal{L}_{j} \rightarrow \mathbb{Z}_{p}$ induce $\Psi: N_{i} \times N_{j} \rightarrow$ $\mathcal{O}_{D}$ such that

$$
\mathcal{E}\left(u_{1} \otimes n_{1}, u_{2} \otimes n_{2}\right)=h\left(u_{1}, u_{2} \Psi\left(n_{1}, n_{2}\right)\right) .
$$

Then $\left\{N_{i}\right\}$ give a polarized chain of $\mathcal{O}_{D}$-lattices in $W$ for the form $\Psi$ and the point here is that there is a uniquely determined polarized lattice chain $N_{\bullet}$ that produces the polarized chain $\mathcal{L}_{\bullet}$ as above.

7.b.3. To proceed we assume that the group $G$ splits over a tamely ramified extension of $\mathbb{Q}_{p}$. In particular, the prime $p$ is at most tamely ramified in the center $F$ of $B$. Now let us explain how we can extend the above construction over the base $\mathbb{Z}_{p}[u]$.

$\left(\right.$ Case A) We set $\mathcal{O}(\mathcal{B})=M_{n}(\mathcal{O}(\mathcal{D})) \times M_{n}\left(\mathcal{O}(\mathcal{D})^{\text {opp }}\right)$ with $\mathcal{O}(\mathcal{D})$ over $W[v]$ as given in (4.5); denote again by $*$ the exchange involution on $\mathcal{O}(\mathcal{B})$. Recall we view $W[v]$ as a $\mathbb{Z}_{p}[u]$-algebra via $u \mapsto v^{e} \cdot\left(p \cdot \varpi^{-e}\right)$. Set $\underline{U}=\mathcal{O}(\mathcal{D})^{n} \otimes_{\mathcal{O}(\mathcal{D})} \underline{T}$ (a left $M_{n}(\mathcal{O}(\mathcal{D})$ )module) where $\underline{T} \simeq \mathcal{O}(\mathcal{D})^{m}$ and $\underline{\widetilde{U}}=\operatorname{Hom}_{\mathbb{Z}_{p}[u]}\left(\underline{U}, \mathbb{Z}_{p}[u]\right)$ which is then also naturally a left $M_{n}\left(\mathcal{O}(\mathcal{D})^{\text {opp }}\right)$-module. Set $\underline{W}=\underline{U} \oplus \underline{\widetilde{U}}$ which is a left $\mathcal{O}(\mathcal{B})$-module; then $\underline{W}$ also supports a unique perfect alternating $\mathbb{Z}_{p}[u]$-bilinear form ( , ) for which $\underline{U}, \underline{\widetilde{U}}$ are isotropic and $((u, 0),(0, \tilde{u}))=\tilde{u}(u)$ for all $u \in \underline{U}, \tilde{u} \in \underline{\tilde{U}}$. Now choose an $\mathcal{O}(\mathcal{D})$-lattice chain $\underline{\Gamma}_{\bullet}$ in $\mathcal{O}(\mathcal{D})^{m}=\oplus_{i=1}^{m} \mathcal{O}(\mathcal{D}) e_{i}$

$$
\underline{\Gamma}_{r}=X \cdot \underline{\Gamma}_{0} \subset \underline{\Gamma}_{r-1} \subset \cdots \subset \underline{\Gamma}_{0}=\mathcal{O}(\mathcal{D})^{m}
$$


such that $\underline{\Gamma}_{j}=\oplus_{j=1}^{m} X^{a_{j}} \mathcal{O}(\mathcal{D})$ for some $1 \geq a_{j} \geq 0$ which lifts the corresponding lattice chain $\Gamma_{j}$. Consider

$$
\underline{U_{\bullet}}=\mathcal{O}(\mathcal{D})^{n} \otimes_{\mathcal{O}(\mathcal{D})} \Gamma_{\bullet} .
$$

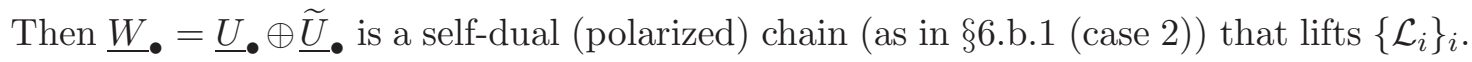
Consider

$$
\mathcal{G}(R)=\operatorname{Aut}_{\mathcal{O}(\mathcal{B}) \otimes_{\mathbb{Z}_{p}[u]} R}\left(\left\{\underline{W} \bullet \otimes_{\mathbb{Z}_{p}[u]} R\right\},(,,)\right)
$$

where the automorphisms of the chain are supposed to preserve the form $($,$) up to$ common similitude in $R^{*}$. As in 4.c.3, 4.c.11, we can see that $\mathcal{G}$ is one of the group schemes constructed in Theorem 3.1. By its construction above, the group scheme $\mathcal{G}$ is a closed subgroup scheme of $\operatorname{GSp}\left(\underline{W}_{\bullet}\right)$.

(Case B) Recall from 4.c.4 our notation of $\mathfrak{O}$ which could be $W[v], \mathfrak{R}$ or $\mathcal{O}(\mathcal{Q})$. The algebra $\mathfrak{O}$ specializes to $\mathcal{O}_{D}$ above under $v \mapsto \varpi_{0}$ (note here that in general the "base field" is $\left.F_{0}\right)$. Notice that the involution $d \mapsto \breve{d}$ of $\mathcal{O}_{D}$ has a canonical extension to an involution of $\mathfrak{O}$; we will denote this involution by the same symbol. Now to start our construction, first extend the form $H$ to a perfect form $\underline{H}: \mathfrak{D}^{n} \times \mathfrak{D}^{n} \rightarrow \mathfrak{O}$ of the type described in 4 s.c such that under $W[v] \rightarrow \mathcal{O}_{F_{0}}, v \mapsto \varpi_{0}$, we obtain a form isomorphic to $H$. For example, suppose we are in case (B1). Then depending on the type of the involution $*$, the form $H$ is either symmetric or alternating. In the alternating case, all perfect forms on $\mathcal{O}_{F}^{n}$ are isomorphic to the standard form and we can extend this over $W[v]$ as in 4. .c.5. In the symmetric case, the discriminant of the perfect form is in $W^{*} /\left(W^{*}\right)^{2} \simeq \mathcal{O}_{F}^{*} /\left(\mathcal{O}_{F}^{*}\right)^{2}$ and the Hasse invariant is trivial; this implies that there are only two cases to consider: the split case in which $H$ is isomorphic to the standard form and the quasi-split unramified case; these can be lifted as in the first cases of \$4.c.6. The other cases can be dealt with by similar arguments using 4. .c.9 and following the pattern explained in [55, Appendix to Ch. 3]. We will leave the details to the reader. Notice that since we are assuming that the form $H$ is perfect on $\mathcal{O}_{D}^{n}$ there are fewer cases to consider. On the other hand, we now also have to allow the anti-hermitian forms and also the quaternionic $\epsilon$-hermitian for the new involution as in $\$$ 4.c.12,

Define now a perfect form $\underline{h}: \mathfrak{O}^{n} \times \mathfrak{O}^{n} \rightarrow \mathbb{Z}_{p}[u]$ as follows: We set $\underline{h}=\operatorname{Tr}_{\mathfrak{O} / \mathbb{Z}_{p}[u]}\left(v^{1-e}\right.$. $\underline{H}$ ) if $\mathfrak{O}=W[v]$ or $\mathfrak{O}=\mathfrak{R}=W_{2}[v]$ (unramified case), $\underline{h}=\operatorname{Tr}_{\mathfrak{O} / \mathbb{Z}_{p}[u]}\left(v^{\prime 1-2 e} \cdot \underline{H}\right)$ if $\mathfrak{O}=$ $\mathfrak{R}=W\left[v^{\prime}\right]$ (ramified case), and finally $\underline{h}=\operatorname{Tr}_{W[v] / \mathbb{Z}_{p}[u]}\left(v^{1-e} \cdot \operatorname{Tr}^{0}\left(X^{-1} \underline{H}\right)\right)$ if $\mathfrak{O}=\mathcal{O}(\mathcal{Q})$.

This construction allows us to extend the involution $*$ on $\mathcal{O}_{B}=M_{n}\left(\mathcal{O}_{D}\right)$ to an involution on $\mathcal{O}(\mathcal{B})=M_{n}(\mathfrak{O})$ which we will also denote by $*$. Indeed, we can set

$$
A^{*}=\underline{C} \cdot{ }^{t} \breve{A} \cdot \underline{C}^{-1}
$$

where $\underline{C}$ is the matrix in $\mathrm{GL}_{n}(\mathfrak{O})$ that gives the perfect form $\underline{H}$. This satisfies $C=\epsilon \cdot{ }^{t} \breve{C}$.

Using 4. .c.9 together with 4 .c.12, we extend $\Psi$ above to an $\epsilon$-hermitian form $\underline{\Psi}$ on $\mathcal{U}=\mathfrak{O}\left[v^{ \pm 1}\right]^{m}$ for the involution $d \mapsto \breve{d}$ of the type described in 4 .C. As before, the form $\underline{\Psi}$ has the opposite parity of that of $\underline{H}$ (i.e if one is $\epsilon$-hermitian the other is $(-\epsilon)$-hermitian).

The self-dual $\mathcal{O}_{D}$-chain $N$ • gives a point $x$ in the building of the corresponding group over $\mathbb{Q}_{p}$. We can assume that $x$ belongs to the apartment of the standard maximal split 
torus; then we can give a self-dual $\mathfrak{O}$-lattice chain $\underline{N}$. in $\mathfrak{O}\left[v^{ \pm 1}\right]^{m}$ that extends the selfdual $\mathcal{O}_{D}$-chain $N_{\bullet}$. This can be done explicitly on a case-by-case basis by appealing to the list of cases in [55. Appendix to Ch. 3]. Alternatively, we can argue as follows: As in 3.a.3, 3.c the identification of apartments induced by $u \mapsto p$, gives a corresponding point $x_{\mathbb{Q}_{p}((u))}$ in the building of $\underline{G}_{\mathbb{Q}_{p}((u))}$ over $\mathbb{Q}_{p}((u))$; this corresponds to a self-dual $\mathbb{Q}_{p}[[u]]$-lattice chain. Now we can use the construction in Remark 4.2 to obtain the desired $\mathfrak{O}$-lattice chain over $\mathbb{Z}_{p}[u]$.

Now consider the tensor products $\underline{M}_{i}=\mathfrak{O}^{n} \otimes \mathfrak{O} \underline{N}_{i}$. These are free (left) $M_{n}(\mathfrak{O})$ modules; they are all contained in $\underline{M}_{i}\left[v^{-1}\right]=\left(\mathfrak{O}^{n} \otimes_{\mathfrak{O}} \mathfrak{D}^{m}\right)\left[v^{-1}\right]$. Define a $\mathbb{Z}_{p}[u]$-valued form $\underline{\mathcal{E}}$ on $\left(\mathfrak{O}^{n} \otimes_{\mathfrak{O}} \mathfrak{O}^{m}\right)\left[v^{-1}\right]$ by

$$
\underline{\mathcal{E}}\left(u_{1} \otimes n_{1}, u_{2} \otimes n_{2}\right)=\underline{h}\left(u_{1}, u_{2} \underline{\Psi}\left(n_{1}, n_{2}\right)\right) .
$$

We can check that the parity condition above implies that the form is alternating, i.e $\underline{\mathcal{E}}(x, y)=-\underline{\mathcal{E}}(y, x)$. Also, the form $\underline{\mathcal{E}}$ satisfies

$$
\underline{\mathcal{E}}\left(b^{*} m_{1}, m_{2}\right)=\underline{\mathcal{E}}\left(m_{1}, b m_{2}\right)
$$

for $b \in M_{n}(\mathfrak{O}), m_{1}, m_{2} \in \underline{M}_{i}\left[v^{-1}\right]$. (Notice that the form $\underline{\Psi}$ is uniquely determined by $\underline{\mathcal{E}}$.) As a result of this construction, we have given in particular, a self-dual chain $\left\{\underline{M}_{i}\right\}_{i}$ of $M_{n}(\mathfrak{O})$-lattices for the involution $*$ and the alternating form $\underline{\mathcal{E}}$ that specializes to the self-dual chain of $M_{n}\left(\mathcal{O}_{D}\right)$-lattices $\{\mathcal{L}\}$ of our initial data.

Now consider the group scheme $\mathcal{G}^{\prime}$ of automorphisms of the self-dual $\mathfrak{O}$-lattice chain $\left(\underline{N}_{i}\right)_{i}$ such that the corresponding automorphism of $\left(\underline{M}_{i}\right)_{i}$ respects the form $\underline{\mathcal{E}}$ up to a common similitude as above. By 4 .c.11 the connected component $\mathcal{G}$ of $\mathcal{G}^{\prime}$ is an example of the group schemes of Theorem 3.1. Our construction provides a group scheme homomorphism

$$
\underline{\rho}: \mathcal{G}^{\prime} \rightarrow \operatorname{GSp}\left(\underline{M}_{\bullet}\right)
$$

As in [55, Appendix to Ch. 3], using Morita equivalence we can see that this is a closed immersion. We can also see that $\underline{\rho}$ extends the natural symplectic representation $\rho: \mathbf{G} \rightarrow$ GSp obtained from the PEL data. It follows that the homomorphism $\underline{\rho}: \mathcal{G} \rightarrow \operatorname{GSp}\left(\underline{M}_{\bullet}\right)$ satisfies the assumptions of the previous section. (Here, we indeed have to allow that $\mathcal{G}$ may not be closed in $\operatorname{GSp}\left(\underline{M}_{\bullet}\right)$. An example is when $G$ is a ramified unitary similitude group on an even number of variables and $x$ a vertex corresponding to a single self-dual lattice for the corresponding hermitian form. Then the fiber over $u=0$ of the Zariski closure of $\underline{G}$ in $\operatorname{GSp}\left(\underline{M_{\bullet}}\right.$ ) has two connected components, see [50, 1.3].)

Using the above together with Proposition 7.1, we now see that in the case of PEL Shimura varieties of [55] we can identify $\mathrm{M}(G,\{\mu\})_{K}=M_{\mathcal{G}, \mu}$ with the Zariski closure of $G / P_{\mu}$ in the symplectic local model $\mathrm{M}\left(\mathrm{GSp}_{2 n}\right)_{M_{\bullet}} \otimes_{\mathcal{O}} \mathcal{O}_{E}$ of [20] under the standard symplectic representation $\rho: \mathbf{G} \rightarrow \mathrm{GSp}_{2 n} 6$

\footnotetext{
${ }^{6}$ We emphasize here that, in general, $\mathbf{G}$ is not connected and that $G=\mathbf{G}_{\mathbb{Q}_{p}}^{\circ}$.
} 
7.b.4. We will now explain how the work of Rapoport and Zink ([55]) combined with the above can be used to produce integral models of PEL Shimura varieties that afford a diagram as in (7.1). Then as a result of Theorem 0.1 , these models satisfy favorable properties, c.f. Theorem 0.2. Our explanation becomes more complicated when the group $\mathbf{G}_{\mathbb{Q}_{p}}$ is not connected, one reason being that our theory of local models has been set up only for connected groups. At first glance, the reader can assume that $\mathbf{G}_{\mathbb{Q}_{p}}$ is connected; then everything simplifies considerably.

We will continue to use some of the notations and constructions of [55]. Starting from the PEL data $\mathfrak{D}=\left(\mathbf{B}, \mathcal{O}_{\mathbf{B}},{ }^{*}, \mathbf{V},(), h,,\{\mathcal{L}\}, K^{p}\right)$ with corresponding group $\mathbf{G}$ and the choice of a prime $\mathfrak{P} \mid(p)$ of the reflex field $\mathbf{E}$, Rapoport and Zink define a moduli functor $\mathcal{A}_{K^{p}}$ over $\mathcal{O}_{E^{b}}=\mathcal{O}_{\mathbf{E}_{\mathfrak{P}}}$ (see [55, Definition 6.9], here $E^{b}=\mathbf{E}_{\mathfrak{P}}$ is the local reflex field). Here $K^{p}$ is a compact open subgroup of $\mathbf{G}\left(\mathbb{A}_{f}^{p}\right)$. When $K^{p}$ is small enough, this functor is representable by a quasi-projective scheme $\mathcal{A}_{K^{p}}$ over $\operatorname{Spec}\left(\mathcal{O}_{E^{b}}\right)$.

Recall $G^{b}=\mathbf{G}_{\mathbb{Q}_{p}}, G=\mathbf{G}_{\mathbb{Q}_{p}}^{\circ}$ (a connected reductive group); as usual, we assume these split over a tamely ramified extension of $\mathbb{Q}_{p}$. The Shimura data give a conjugacy class of cocharacters $\mu: \mathbb{G}_{\mathrm{m} \overline{\mathbb{Q}}_{p}} \rightarrow G_{\overline{\mathbb{Q}}_{p}}^{b}$; then $E^{b}$ is the field of definition of this conjugacy class. Denote by $K_{p}^{b}$ the stabilizer of the lattice chain $\{\mathcal{L}\}$ in $G^{b}\left(\mathbb{Q}_{p}\right)=\mathbf{G}\left(\mathbb{Q}_{p}\right)$. Set $\mathbf{K}^{b}=K^{p} \cdot K_{p}^{b}$. Then the generic fiber $\mathcal{A}_{K^{p}} \otimes_{\mathcal{O}_{E^{b}}} E^{b}$ contains the Shimura variety $S h_{\mathbf{K}^{b}} \otimes_{\mathbf{E}} E^{b}$ for $\mathbf{G}$, as a union of some of its connected components; $\mathcal{A}_{K^{p}} \otimes_{\mathcal{O}_{E^{b}}} E^{b}$ could also contain more Shimura varieties, which correspond to other forms of the group $\mathbf{G}$ (for example, when the Hasse principle fails c.f. [36]). Set $K_{p}^{\prime}=K_{p}^{b} \cap G\left(\mathbb{Q}_{p}\right)$ and denote by $K=K_{p}$ the parahoric subgroup of $G\left(\mathbb{Q}_{p}\right)$ that corresponds to $x(\mathcal{L})$. Recall we denote by $\mathcal{P}$ the corresponding (connected) smooth group scheme over $\mathbb{Z}_{p}$ and by $\mathcal{P}^{\prime}$ the smooth group scheme over $\mathbb{Z}_{p}$ determined by the stabilizer of $x(\mathcal{L})$ so that $K_{p}^{\prime}=\mathcal{P}^{\prime}\left(\mathbb{Z}_{p}\right) ; \mathcal{P}$ is the neutral component of $\mathcal{P}^{\prime}$. Then $K=K_{p}$ is a normal subgroup of finite index in $K_{p}^{\prime}$. (In most cases, we have $K_{p}^{\prime}=K_{p}, \mathcal{P}^{\prime}=\mathcal{P}$.) Also recall that we denote by $\mathcal{P}^{b}$ the smooth group scheme of $\mathcal{O}_{B}$-isomorphisms of the polarized multichain $\{\mathcal{L}\}$ up to common similitude; we have $\mathcal{P}^{b} \otimes_{\mathbb{Z}_{p}} \mathbb{Q}_{p}=G^{b}$

By [55], we have a smooth morphism of algebraic stacks over $\operatorname{Spec}\left(\mathcal{O}_{E^{b}}\right)$

$$
\varphi: \mathcal{A}_{K^{p}} \rightarrow\left[\mathrm{M}^{\text {naive }} / \mathcal{P}_{\mathcal{O}_{E^{b}}^{b}}\right]
$$

where $\mathrm{M}^{\text {naive }}$ is the "naive" local model that corresponds to our data (see loc. cit. Def. 3.27 , where this is denoted by $\left.\mathrm{M}^{\mathrm{loc}}\right)$. By its definition, $\mathrm{M}^{\text {naive }}$ is a closed subscheme of the symplectic local model $\mathrm{M}\left(\mathrm{GSp}_{2 n}\right)_{M_{\bullet}} \otimes_{\mathbb{Z}_{p}} \mathcal{O}_{E^{\mathrm{b}}}$ as above.

The generic fiber of $\mathrm{M}^{\text {naive }}$ is a projective homogeneous space over $E^{b}$ for the group $G^{b}$ (which is not always connected). Observe that each cocharacter $\mu: \mathbb{G}_{\mathrm{m}} \overline{\mathbb{Q}}_{p} \rightarrow G_{\overline{\mathbb{Q}}_{p}}^{b}$ lands in the neutral component $G_{\overline{\mathbb{Q}}_{p}}$. Let $\left\{\mu_{i}\right\}_{i}$ be a set of representatives (up to $G\left(\overline{\mathbb{Q}}_{p}\right)$ conjugation) of the cocharacters of $G_{\overline{\mathbb{Q}}_{p}}$ in the $G^{b}\left(\overline{\mathbb{Q}}_{p}\right)$-conjugacy class of $\mu$; we can write $\mu_{i}=\tau_{i} \mu \tau_{i}^{-1}$ with $\tau_{i} \in G^{b}\left(\overline{\mathbb{Q}}_{p}\right)$. Denote by $E_{i}$ the field of definition of the $G\left(\overline{\mathbb{Q}}_{p}\right)$ conjugacy class of $\mu_{i}$. Then $E^{b} \subset E_{i}$. The generic fiber $\mathrm{M}\left(G,\left\{\mu_{i}\right\}\right)_{K} \otimes_{\mathcal{O}_{E_{i}}} E_{i}=G_{E_{i}} / P_{\mu_{i}}$ is a homogeneous space for $G_{E_{i}}$. By the above and Proposition 7.1, we obtain closed 
immersions

$$
\iota_{i}: \mathrm{M}\left(G,\left\{\mu_{i}\right\}\right)_{K} \rightarrow \mathrm{M}^{\text {naive }} \otimes_{\mathcal{O}_{E^{b}}} \mathcal{O}_{E_{i}}
$$

of schemes over $\mathcal{O}_{E_{i}}$ which are equivariant for the action of the group scheme $\mathcal{P}_{\mathcal{O}_{E_{i}}}$. In fact, since $\mathcal{P}_{\mathcal{O}_{E_{i}}}^{\prime}$ is a closed subgroup scheme of $\operatorname{GSp}\left(M_{\bullet}\right)$, the action of $\mathcal{P}_{\mathcal{O}_{E_{i}}}$ on $\mathrm{M}\left(G,\left\{\mu_{i}\right\}\right)_{K}$ extends to an action of $\mathcal{P}_{\mathcal{O}_{E_{i}}}^{\prime}$ such that $\iota_{i}$ remains equivariant. Now let $\tilde{E}$ be a Galois extension of $\mathbb{Q}_{p}$ that splits $G$ and contains all the fields $E_{i}$. Then the base change $\mathrm{M}^{\text {naive }} \otimes_{\mathcal{O}_{E^{b}}} \tilde{E}$ is a disjoint union

$$
\mathrm{M}^{\text {naive }} \otimes_{\mathcal{O}_{E^{b}}} \tilde{E}=\bigsqcup_{i}\left(\mathrm{M}\left(G,\left\{\mu_{i}\right\}\right)_{K} \otimes_{\mathcal{O}_{E_{i}}} \tilde{E}\right)=\bigsqcup_{i} G_{E_{i}} / P_{\mu_{i}} \otimes_{E_{i}} \tilde{E}
$$

We obtain a morphism

$$
\iota: N:=\bigsqcup_{i} \mathrm{M}\left(G,\left\{\mu_{i}\right\}\right)_{K} \otimes_{\mathcal{O}_{E_{i}}} \mathcal{O}_{\tilde{E}} \rightarrow \mathrm{M}^{\text {naive }} \otimes_{\mathcal{O}_{E^{b}}} \mathcal{O}_{\tilde{E}}
$$

of schemes over $\mathcal{O}_{\tilde{E}}$. The scheme $N$ supports an action of $\mathcal{P}_{\mathcal{O}_{\tilde{E}}}^{\prime}$ while its generic fiber supports a compatible action of $G_{\tilde{E}}^{b}$; we can see that the action of $\mathcal{P}_{\mathcal{O}_{\tilde{E}}}^{\prime}$ on $N$ extends to an action of $\mathcal{P}_{\mathcal{O}_{\tilde{E}}}^{b}$ such that $\iota$ is $\mathcal{P}_{\mathcal{O}_{\tilde{E}}}^{b}$-equivariant. Both the source and target of $\iota$ (considered as schemes over $\left.\mathcal{O}_{E^{b}}\right)$ support an action of the Galois group $\Gamma=\operatorname{Gal}\left(\tilde{E} / E^{b}\right)$ and $\iota$ is $\Gamma$-equivariant (we can check these statements by looking at the generic fibers).

In this more general situation, we define the local model to be the quotient

$$
\mathrm{M}^{\mathrm{loc}}=N / \Gamma \text {. }
$$

This is a flat $\mathcal{O}_{E^{b}}$-scheme. The morphism $\iota$ gives

$$
\xi: \mathrm{M}^{\text {loc }}=N / \Gamma \rightarrow\left(\mathrm{M}^{\text {naive }} \otimes_{\mathcal{O}_{E^{b}}} \mathcal{O}_{\tilde{E}}\right) / \Gamma=\mathrm{M}^{\text {naive }}
$$

which we can check is $\mathcal{P}_{\mathcal{O}_{E^{b}}}^{b}$-equivariant and gives an isomorphism between generic fibers.

Remark 7.2. If $\mathbf{G}$ is connected, then $G=G^{b}$, there is only one $i$ and $E=E_{i}=E^{b}$ is the local reflex field as above. In that case, $\mathrm{M}^{\mathrm{loc}}=\mathrm{M}(G,\{\mu\})_{K}$.

Pulling back the smooth $\phi(7.10)$ along $\xi$ gives a cartesian diagram

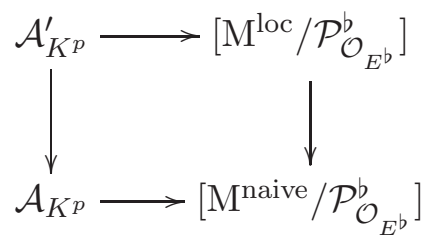

with both horizontal arrows smooth.

The schemes $\mathcal{A}_{K^{p}}^{\prime}$ and $\mathcal{A}_{K^{p}}$ have isomorphic generic fibers. Using the arguments in [36, §8] applied to $\mathcal{A}_{K^{p}}^{\prime} \otimes_{\mathcal{O}_{E^{b}}} E^{b}=\mathcal{A}_{K^{p}} \otimes_{\mathcal{O}_{E^{b}}} E^{b}$ we can now express this scheme as a union of Shimura varieties $S h_{\mathbf{K}^{b}}^{(j)}$ given by various forms $\mathbf{G}^{(j)}$ of the group $\mathbf{G} 7$ (These forms satisfy $\mathbf{G}^{(j)}\left(\mathbb{Q}_{v}\right) \simeq \mathbf{G}\left(\mathbb{Q}_{v}\right)$, for all places $\left.v \neq p\right)$. Therefore, $\mathcal{A}_{K^{p}}^{\prime}$ is a flat model over $\mathcal{O}_{E^{b}}$ for such a union of Shimura varieties.

\footnotetext{
${ }^{7}$ For this, we need to allow in our formalism Shimura varieties for some non-connected reductive groups.
} 
Since we assume that $p$ is odd, we have $p \nmid\left|\pi_{1}\left(G_{\text {der }}\right)\right|$. Theorem 8.1 and Proposition 8.2 below imply that the base changes $\mathrm{M}\left(G,\left\{\mu_{i}\right\}\right)_{K} \otimes_{\mathcal{O}_{E}} \mathcal{O}_{\tilde{E}}$ are normal. Since normality is preserved by taking invariants by a finite group, $\mathrm{M}^{\mathrm{loc}}=N / \Gamma$ is also normal. It follows that the strict henselizations of $\mathrm{M}^{\mathrm{loc}}$ at all closed points are normal and therefore also integral. We can now conclude, using the smoothness of $\phi: \mathcal{A}_{K^{p}}^{\prime} \rightarrow\left[\mathrm{M}^{\mathrm{loc}} / \mathcal{P}_{\mathcal{O}_{E^{b}}}^{b}\right]$, that the same is true for the strict henselizations of $\mathcal{A}_{K^{p}}^{\prime}$. It follows that the Zariski closures of $S h_{\mathbf{K}^{b}}^{(j)}$ in $\mathcal{A}_{K^{p}}^{\prime}$ do not intersect; denote these (reduced) Zariski closures by $\mathcal{S}_{\mathbf{K}^{b}}^{(j)}$. They are integral models of the Shimura varieties $S h_{\mathbf{K}^{b}}^{(j)}$. Then, for each $j$, the morphism

$$
\phi: \mathcal{S}_{\mathbf{K}^{\mathrm{b}}}^{(j)} \rightarrow\left[\mathrm{M}^{\mathrm{loc}} / \mathcal{P}_{\mathcal{O}_{E^{b}}}^{b}\right]
$$

obtained by restricting $\phi$, is also smooth. Hence, $\mathrm{M}^{\mathrm{loc}}$ is also a "local model" for the integral models $\mathcal{S}_{\mathbf{K}^{b}}^{(j)}$ of the Shimura varieties $S h_{\mathbf{K}^{b}}^{(j)}$. In most cases, the structure of $\mathrm{M}^{\text {loc }}$ and therefore also the local structure of $\mathcal{S}_{\mathbf{K}^{b}}^{(j)}$ can be understood using Theorem 0.1 . We will explain this below.

Remark 7.3. We can see as in [36] that, in the situation above, all the forms $\mathbf{G}^{(j)}$ that appear in the list satisfy $\mathbf{G}^{(j)}\left(\breve{\mathbb{Q}}_{p}\right) \simeq \mathbf{G}\left(\breve{\mathbb{Q}}_{p}\right)$.

(a) Suppose now that, in addition, at least one of the following two conditions is satisfied:

(p1) the special fiber of $\mathcal{P}^{b}$ is connected, i.e $K_{p}^{b}$ is parahoric, or

(p2) the sheaf of connected components $\mathcal{P}^{b} /\left(\mathcal{P}^{b}\right)^{\circ}$ is étale locally constant and its generic fiber is isomorphic (via the natural map) to $G^{b} /\left(G^{b}\right)^{\circ}$.

Then, the arguments of [36, Lemma 7.2 and $\S 8]$ extend to show that all the groups $\mathbf{G}^{(j)}$ also satisfy $\mathbf{G}^{(j)}\left(\mathbb{Q}_{p}\right) \simeq \mathbf{G}\left(\mathbb{Q}_{p}\right)$. (Indeed, then one can apply Lang's theorem as in the proof of Lemma 7.2 loc. cit. since the special fiber of $\left(\mathcal{P}^{b}\right)^{\circ}$ is connected.)

(b) Assume that, in addition to (p1) or (p2), G satisfies the Hasse principle, i.e $\mathrm{H}^{1}(\mathbb{Q}, \mathbf{G}) \rightarrow \prod_{v} \mathrm{H}^{1}\left(\mathbb{Q}_{v}, \mathbf{G}\right)$ is injective. Then the argument of [36, $\left.\S 8\right]$ shows that there is only one Shimura variety for the group $\mathbf{G}^{(j)}=\mathbf{G}$ that appears in the generic fiber of $\mathcal{A}_{K_{p}}$. In this case, we just take $\mathcal{S}_{\mathbf{K}^{b}}=\mathcal{A}_{K^{p}}^{\prime}$.

7.b.5. Here we explain how we can combine the local model diagram (17.12) with Theorem 0.1 to give results on integral models of PEL Shimura varieties. In particular, we show Theorem 0.2 .

a) The set-up simplifies drastically if $G^{b}$ is connected, i.e if $G=G^{b}$. This is the case when $\mathbf{G}$ does not have any orthogonal factors. Then $\mathrm{M}^{\text {loc }}=\mathrm{M}(G,\{\mu\})_{K}(\Gamma=\{1\})$ and $\xi=\iota$ is a closed immersion. Also, in this case the derived group $G_{\text {der }}$ is simplyconnected. We can then use Theorem 0.1 and the above directly to obtain information on the integral models $\mathcal{A}_{K^{p}}^{\prime}$ and $\mathcal{S}_{\mathbf{K}^{b}}^{(j)}$. Assume in addition that $K_{p}^{b}$ is parahoric, i.e that the special fiber of $\mathcal{P}^{b}=\mathcal{P}^{\prime}$ is connected. Then the existence of the local model diagram morphism $\phi$ of (7.12) together with the results in 98 , and in particular Theorem 0.1, implies, in a standard way, that the integral models $\mathcal{A}_{K^{p}}^{\prime}$ and $\mathcal{S}_{\mathbf{K}^{b}}^{(j)}$ satisfy the conclusions of the statement of Theorem 0.2 . (Notice here that in the statement of Theorem 0.2 
we refer somewhat ambiguously to the "Shimura variety $S h_{\mathbf{K}}$ defined by the PEL data $\mathfrak{D}$ ". If the Hasse principle is not satisfied, $S h_{\mathbf{K}}$ is by definition the generic fiber of $\mathcal{A}_{K^{p}}^{\prime}$ which is a union of several Shimura varieties; by the above, $\mathcal{S}_{\mathbf{K}^{b}}^{(j)}$ give integral models as in Theorem 0.2 for each one of them.)

b) Continue to assume that $G=G^{b}$. In general, if $\mathcal{P}^{\prime}=\mathcal{P}^{b}$ is not connected, $K_{p}^{\prime} / K_{p}$ is a non-trivial abelian group. The above allows us to understand the structure of integral models of Shimura varieties with $K_{p}^{\prime}$-level structure. With some extra work, one should be able to extend this and also produce local model diagrams for Shimura varieties with $K_{p}$-level structure. We will not discuss this in this paper. See [50, 1.3] for an example where a case of $K_{p}$-level structure for the ramified unitary group is discussed.

c) Let us now consider the general case, i.e allow $G \neq G^{b}$. First of all, let us remark that the scheme $\mathrm{M}^{\text {loc }}$ defined above coincides in various special cases with the "corrected" local model as considered in [47, 48], [50, [67, 51]. Indeed, first assume that $G=G^{b}, E=E^{b}$ as above. Then, for both our definition above and the definition in these references, $\mathrm{M}^{\text {loc }}$ is simply the Zariski closure of $G_{E} / P_{\mu}$ in $\mathrm{M}^{\text {naive }}$. Now let us allow $G \neq G^{b}$; such cases have not been studied extensively before and one needs to work a bit harder. Some (even) orthogonal similitude group cases where $G \neq G^{b}$ have been considered in [50, 67] (see [51]). In the split orthogonal case, $E=E^{b}=\mathbb{Q}_{p}$, and it makes sense to define the corrected local model as the Zariski closure of $G^{b} / P_{\mu}=\left(G / P_{\mu_{1}}\right) \sqcup\left(G / P_{\mu_{2}}\right)$ in $\mathrm{M}^{\text {naive}}$; it follows from [50, §8.2] that this Zariski closure is the disjoint union of the closures of $G / P_{\mu_{1}}$ and $G / P_{\mu_{2}}$. This coincides with the description of $\mathrm{M}^{\text {loc }}$ above and we have $\mathrm{M}^{\text {loc }}=\mathrm{M}\left(G,\left\{\mu_{1}\right\}\right)_{K} \sqcup \mathrm{M}\left(G,\left\{\mu_{2}\right\}\right)_{K}$. This local model was studied in the Iwahori case by Smithling in [67]. In general, the suggested correction of the naive local model in this orthogonal case [50] involves the so-called "spin condition" whose main virtue is that it also attempts to give a moduli theoretic interpretation. (The spin condition also makes sense in the quasi-split even orthogonal case $([50, \S 8.2],[51, \S 2.7])$. We conjecture that the corresponding local model always agrees with our definition above.)

As an example of a result we can obtain when $G \neq G^{b}$, let us suppose that $G^{b}$ is a split even orthogonal similitude group as above and that $\mathcal{L}$ gives a maximal self-dual lattice chain (the Iwahori case considered in [67]). Then the special fiber of $\mathcal{P}^{b}$ is connected. By (7.12) and the above, the local model $\mathrm{M}^{\mathrm{loc}}=\mathrm{M}\left(G,\left\{\mu_{1}\right\}\right)_{K} \sqcup \mathrm{M}\left(G,\left\{\mu_{2}\right\}\right)_{K}$ describes the singularities of corresponding integral models of "orthogonal" PEL Shimura varieties. Theorem 0.1 then also implies that these integral models are normal and have reduced special fibers with geometric components which are normal and Cohen-Macaulay. In the general case that $G \neq G^{b}$, one would need to study the quotient $N / \Gamma=\mathrm{M}^{\text {loc }}$ but we will leave this for another occasion.

d) For general Shimura varieties of abelian type, the existence of a suitable integral model and a local model diagram as above is the subject of joint work in progress by the first named author and M. Kisin [31]. 


\section{THE SPECIAL FIBERS OF THE LOCAL MODELS}

Here we show our main results on the structure of the local models, including Theorem 0.1 of the introduction.

8.a. Affine Schubert varieties and the $\mu$-admissible set. Let us review some aspects of the theory of Schubert varieties in the (generalized) affine flag varieties, following [16, 49]. In this section, we assume that $F^{\prime}=k((u)), \mathcal{O}^{\prime}=k[[u]]$, with $k$ algebraically closed, and let $G^{\prime}$ be a connected reductive group over $F^{\prime}$, split over a tamely ramified extension $\tilde{F}^{\prime}$ of $F^{\prime}$.

8.a.1. Let $S^{\prime}$ be a maximal $F^{\prime}$-split torus of $G^{\prime}$ and $T^{\prime}=Z_{G^{\prime}}\left(S^{\prime}\right)$ a maximal torus. Let $I=\operatorname{Gal}\left(\tilde{F}^{\prime} / F^{\prime}\right)$. Let $x \in \mathcal{B}\left(G^{\prime}, F^{\prime}\right)$ be a point in the building, which we assume lies in the apartment $\mathcal{A}\left(G^{\prime}, S^{\prime}, F^{\prime}\right)$ and let $\mathcal{P}_{x}^{\prime}$ be the corresponding parahoric group scheme over $k[[u]]$. Let $\operatorname{Gr}_{\mathcal{P}_{x}^{\prime}}$ be the affine Grassmannian. Then $L^{+} \mathcal{P}_{x}^{\prime}$ acts on $\operatorname{Gr}_{\mathcal{P}_{x}^{\prime}}$ via left multiplication. The orbits then are parameterized by $W^{\mathcal{P}_{x}^{\prime}} \backslash \widetilde{W^{\prime}} / W^{\mathcal{P}_{x}^{\prime}}$, where $\widetilde{W^{\prime}}$ is the Iwahori-Weyl group of $G^{\prime}$ and $W^{\mathcal{P}_{x}^{\prime}}$ is the Weyl group of $\mathcal{P}_{x}^{\prime} \otimes k$. For $w \in W^{\mathcal{P}_{x}^{\prime}} \backslash \widetilde{W^{\prime}} / W^{\mathcal{P}_{x}^{\prime}}$, let $S_{w}^{\mathcal{P}_{x}^{\prime}} \subset \operatorname{Gr}_{\mathcal{P}_{x}^{\prime}}$ denote the corresponding Schubert variety, i.e. the closure of the $L^{+} \mathcal{P}_{x}^{\prime}$ orbit through $w$. Then according to [16, [49, Thm. 8.4], if char $k \nmid\left|\pi_{1}\left(G_{\text {der }}^{\prime}\right)\right|$, where $G_{\text {der }}^{\prime}$ is the derived group of $G^{\prime}$, then $S_{w}^{\mathcal{P}_{x}^{\prime}}$ is normal, has rational singularities and is Frobenius-split if char $k>0$.

Let us also recall the structure of the Iwahori-Weyl group $\widetilde{W}^{\prime}$ of $G^{\prime}$. It is defined via the exact sequence

$$
1 \rightarrow T^{\prime}\left(\mathcal{O}^{\prime}\right) \rightarrow N^{\prime}\left(F^{\prime}\right) \rightarrow \widetilde{W^{\prime}} \rightarrow 1
$$

(where $T^{\prime}\left(\mathcal{O}^{\prime}\right)$ is the group of $\mathcal{O}^{\prime}$-valued points of the unique Iwahori group scheme for the torus $\left.T^{\prime}\right)$ and acts on $\mathcal{A}\left(G^{\prime}, S^{\prime}, F^{\prime}\right)$ via affine transformations. In addition, there is a short exact sequence

$$
1 \rightarrow \mathbb{X}_{\bullet}\left(T^{\prime}\right)_{I} \rightarrow \widetilde{W^{\prime}} \rightarrow W_{0}^{\prime} \rightarrow 1
$$

where $W_{0}^{\prime}=N^{\prime}\left(F^{\prime}\right) / T^{\prime}\left(F^{\prime}\right)$ is the relative Weyl group of $G^{\prime}$ over $F^{\prime}$. In what follows, we use $t_{\lambda}$ to denote the translation element in $\widetilde{W^{\prime}}$ given by $\lambda \in \mathbb{X}_{\bullet}\left(T^{\prime}\right)_{I}$ from the above map 8.14) 8. But occasionally, if no confusion is likely to arise, we will also use $\lambda$ itself to denote this translation element. A choice of a special vertex $v$ of $\mathcal{A}\left(G^{\prime}, S^{\prime}, F^{\prime}\right)$ gives a splitting of the above exact sequence and then we can write $w=t_{\lambda} w_{f}$ for $\lambda \in \mathbb{X}_{\bullet}\left(T^{\prime}\right)_{I}$ and $w_{f} \in W_{0}^{\prime}$.

Let us choose a rational Borel subgroup $B^{\prime}$ of $G^{\prime}$ containing $T^{\prime}$. This determines a set of positive roots $\Phi^{+}=\Phi\left(G^{\prime}, S^{\prime}\right)^{+}$for $G^{\prime}$. There is a natural map $\mathbb{X}_{\bullet}\left(T^{\prime}\right)_{I} \rightarrow \mathbb{X}_{\bullet}\left(S^{\prime}\right)_{\mathbb{R}}$. We define

$$
\mathbb{X}_{\bullet}\left(T^{\prime}\right)_{I}^{+}=\left\{\lambda \mid(\lambda, a) \geq 0 \text { for } a \in \Phi^{+}\right\}
$$

\footnotetext{
${ }^{8}$ Note that under the sign convention of the Kottwitz homomorphism in [37], $t_{\lambda}$ acts on $\mathcal{A}\left(G^{\prime}, S^{\prime}, F^{\prime}\right)$ by $v \mapsto v-\lambda$.
} 
Observe that the chosen special vertex $v$ and the rational Borel $B^{\prime}$ determine a unique alcove $C$ in $\mathcal{A}\left(G^{\prime}, S^{\prime}, F^{\prime}\right)$. Namely, we identify $\mathcal{A}\left(G^{\prime}, S^{\prime}, F^{\prime}\right)$ with $\mathbb{X}_{\bullet}\left(S^{\prime}\right)_{\mathbb{R}}$ by $v$ and then $C$ is the unique alcove whose closure contains $v$, and is contained in the finite Weyl chamber determined by $B^{\prime}$.

Let $W_{\text {aff }}^{\prime}$ be the affine Weyl group of $G^{\prime}$, i.e. the Iwahori-Weyl group of $G_{\mathrm{sc}}^{\prime}$, the simply-connected cover of $G_{\mathrm{der}}^{\prime}$. This is a Coxeter group. One has

$$
1 \rightarrow \mathbb{X}_{\bullet}\left(T_{\mathrm{sc}}^{\prime}\right)_{I} \rightarrow W_{\mathrm{aff}}^{\prime} \rightarrow W_{0}^{\prime} \rightarrow 1,
$$

where $T_{\mathrm{sc}}^{\prime}$ is the inverse image of $T^{\prime}$ in $G_{\mathrm{sc}}^{\prime}$. One can write $\widetilde{W^{\prime}}=W_{\text {aff }}^{\prime} \rtimes \Omega^{\prime}$, where $\Omega^{\prime}$ is the subgroup of $\widetilde{W}^{\prime}$ that fixes the chosen alcove $C$. This gives $\widetilde{W}^{\prime}$ a quasi Coxeter group structure; it makes sense to talk about the length of an element $w \in \widetilde{W}^{\prime}$ and there is a Bruhat order on $\widetilde{W^{\prime}}$. Namely, if we write $w_{1}=w_{1}^{\prime} \tau_{1}, w_{2}=w_{2}^{\prime} \tau_{2}$ with $w_{i}^{\prime} \in W_{\text {aff }}^{\prime}, \tau_{i} \in \Omega^{\prime}$, then $\ell\left(w_{i}\right)=\ell\left(w_{i}^{\prime}\right)$ and $w_{1} \leq w_{2}$ if and only if $\tau_{1}=\tau_{2}$ and $w_{1}^{\prime} \leq w_{2}^{\prime}$.

8.a.2. Now let us recall the definition of the $\mu$-admissible set in the Iwahori-Weyl group (cf. [38, see also [51]). We continue with the above notations. Let $\bar{W}^{\prime}$ be the absolute Weyl group of $G^{\prime}$, i.e. the Weyl group for $\left(G_{\tilde{F}^{\prime}}^{\prime}, T_{\tilde{F}^{\prime}}^{\prime}\right)$. Let $\mu:\left(\mathbb{G}_{m}\right)_{\tilde{F}^{\prime}} \rightarrow G^{\prime} \otimes_{F^{\prime}} \tilde{F}^{\prime}$ be a geometrical conjugacy class of 1-parameter subgroups. It determines a $\bar{W}^{\prime}$-orbit in $\mathbb{X}_{\bullet}\left(T^{\prime}\right)$. One can associate to $\mu$ a $W_{0}^{\prime}$-orbit $\Lambda$ in $\mathbb{X}_{\bullet}\left(T^{\prime}\right)_{I}$ as follows. Choose a Borel subgroup of $G^{\prime}$ containing $T^{\prime}$, and defined over $F^{\prime}$. This gives a unique element in this $\bar{W}^{\prime}$ orbit, still denoted by $\mu$, which is dominant with respect to this Borel subgroup. Let $\bar{\mu}$ be its image in $\mathbb{X}_{\bullet}\left(T^{\prime}\right)_{I}$, and let $\Lambda=W_{0}^{\prime} \bar{\mu}$. It turns out that $\Lambda$ does not depend on the choice of the rational Borel subgroup of $G^{\prime}$, since any two such $F^{\prime}$-rational Borel subgroups that contain $T^{\prime}$ are conjugate to each other by an element in $W_{0}^{\prime}$. For $\mu \in \mathbb{X}_{\bullet}\left(T^{\prime}\right)$, define the admissible set

$$
\operatorname{Adm}(\mu)=\left\{w \in \widetilde{W^{\prime}} \mid w \leq t_{\lambda}, \text { for some } \lambda \in \Lambda\right\},
$$

and more generally,

$$
\operatorname{Adm}^{\mathcal{P}_{x}^{\prime}}(\mu)=W^{\mathcal{P}_{x}^{\prime}} \operatorname{Adm}(\mu) W^{\mathcal{P}_{x}^{\prime}} .
$$

If char $k \nmid\left|\pi_{1}\left(G_{\text {der }}^{\prime}\right)\right|$, let us define a reduced closed subvariety of $\operatorname{Gr}_{\mathcal{P}_{x}^{\prime}}$ whose underlying set is given by

$$
\mathcal{A}^{\mathcal{P}_{x}^{\prime}}(\mu)=\bigcup_{w \in \operatorname{Adm}(\mu)} L^{+} \mathcal{P}_{x}^{\prime} w L^{+} \mathcal{P}_{x}^{\prime} / L^{+} \mathcal{P}_{x}^{\prime}=\underset{w \in W^{\mathcal{P}_{x}^{\prime}} \backslash \operatorname{Adm}^{\mathcal{P}_{x}^{\prime}}(\mu) / W^{\mathcal{P}_{x}^{\prime}}}{S_{w}^{\mathcal{P}_{x}^{\prime}}}
$$

In general, we can define a slightly different variety $\mathcal{A}^{\mathcal{P}_{x}^{\prime}}(\mu)^{\circ}$ as in [49, Sect. 10] (see also [76, Sect. 2.2]), which is isomorphic to $\mathcal{A}^{\mathcal{P}_{x}^{\prime}}(\mu)$ when char $k \nmid\left|\pi_{1}\left(G_{\text {der }}^{\prime}\right)\right|$. (Here, we write $\left|\pi_{1}\left(G_{\text {der }}^{\prime}\right)\right|$ for the order of the algebraic fundamental group of $\left.G_{\text {der }}^{\prime}(\overline{k((u))})^{s}\right)$. In fact, when char $k \nmid\left|\pi_{1}\left(G_{\text {der }}^{\prime}\right)\right|, \mathcal{A}^{\mathcal{P}_{x}^{\prime}}(\mu)^{\circ}$ is the translation of $\mathcal{A}^{\mathcal{P}_{x}^{\prime}}(\mu)$ back to the "neutral connected component" of $\operatorname{Gr}_{\mathcal{P}_{x}^{\prime}}$ by a certain element of $G^{\prime}(k((u)))$. The variety $\mathcal{A}^{\mathcal{P}_{x}^{\prime}}(\mu)^{\circ}$ depends only on $G_{\mathrm{ad}}^{\prime}$ and the image of $\mu$ under $G^{\prime} \rightarrow G_{\mathrm{ad}}^{\prime}$. From its definition (loc. cit.), if we have the decomposition $G^{\prime}=G_{1}^{\prime} \times G_{2}^{\prime}, \mu=\mu_{1}+\mu_{2}$ and $\mathcal{P}_{x}^{\prime}=\left(\mathcal{P}_{x}^{\prime}\right)_{1} \times\left(\mathcal{P}_{x}^{\prime}\right)_{2}$, then

$$
\mathcal{A}^{\mathcal{P}_{x}^{\prime}}(\mu)^{\circ} \simeq \mathcal{A}^{\left(\mathcal{P}_{x}^{\prime}\right)_{1}}\left(\mu_{1}\right)^{\circ} \times \mathcal{A}^{\left(\mathcal{P}_{x}^{\prime}\right)_{2}}\left(\mu_{2}\right)^{\circ}
$$


8.b. Special fibers. Let us return to the local models $M_{\mathcal{G}, \mu}$ and show Theorem 0.1 of the introduction. We will assume throughout that $p \nmid\left|\pi_{1}\left(G_{\mathrm{der}}\right)\right|$.

8.b.1. We denote by $\bar{M}_{\mathcal{G}, \mu}=M_{\mathcal{G}, \mu} \otimes_{\mathcal{O}_{E}} k_{E}$ the special fiber of $M_{\mathcal{G}, \mu} \rightarrow \operatorname{Spec}\left(\mathcal{O}_{E}\right)$ over the residue field $k_{E}$ of $\mathcal{O}_{E}$.

Theorem 8.1. Suppose that $p \nmid\left|\pi_{1}\left(G_{\mathrm{der}}\right)\right|$. Then the scheme $M_{\mathcal{G}, \mu}$ is normal. In addition, the special fiber $\bar{M}_{\mathcal{G}, \mu}$ is reduced, and each geometric irreducible component of $\bar{M}_{\mathcal{G}, \mu}$ is normal, Cohen-Macaulay and Frobenius split.

Notice that the set-up now is more general than in Theorem 0.1 , since here $\mu$ is not necessarily minuscule. Recall that by its construction, $\bar{M}_{\mathcal{G}, \mu}$ is a closed subscheme of $\mathrm{Gr}_{P_{k_{E}}}$. Clearly, it is enough to prove the theorem after base changing to $\breve{\mathcal{O}}_{E}$ with residue field $\bar{k}$. Then the second part of Theorem 8.1 that refers to the special fiber $\bar{M}_{\mathcal{G}, \mu}$ is a corollary of the aforementioned results of [16, 49] combined with Theorem 8.3 below which gives a precise description of the geometric special fiber $\bar{M}_{\mathcal{G}, \mu} \otimes_{k_{E}} \bar{k}$ as a union of affine Schubert varieties. We will first explain how this second part also implies the first part, i.e the normality of $M_{\mathcal{G}, \mu}$. This follows from Proposition 8.2 below together with the fact that the generic fiber of $M_{\mathcal{G}, \mu}$ is normal (since it is given by the single affine Schubert variety $X_{\mu}$ associated to $\mu$ ).

Proposition 8.2. Suppose that $Y \rightarrow \operatorname{Spec}(\mathcal{O})$ is a flat scheme of finite type with normal generic fiber and reduced special fiber. Then $Y$ is normal.

Proof. By Serre's criterion, it is enough to check that $Y$ satisfies properties (R1) and (S2). By assumption, these properties are satisfied by the generic fiber $Y \otimes_{\mathcal{O}} F$. Since the special fiber $\bar{Y}=Y \otimes_{\mathcal{O}} k$ is a reduced scheme of finite type and $k$ is perfect, $\bar{Y}$ is generically smooth over $k$. It follows that $Y$ is regular in codimension 1, i.e it satisfies (R1). It remains to show that $Y$ has depth $\geq 2$ at points of codimension $\geq 2$ which are supported on the special fiber. Since $Y$ is flat over $\operatorname{Spec}(\mathcal{O})$, the uniformizer $\varpi$ provides the start of a regular sequence; we can always obtain one additional element in this regular sequence since $\bar{Y}$ is reduced and hence it satisfies (S1).

8.b.2. Here we show the second part of Theorem 8.1. In what follows, the notations are as in 5.b.5. In particular, we have $P_{\bar{k}}=\mathcal{P}_{x_{\bar{k}((u))}}$; this is a parahoric group scheme which is obtained by base-changing $\mathcal{G}$ to $\bar{k}[[u]]$. (The corresponding reductive group is $G^{\prime}=G_{\bar{k}}=\mathcal{G} \times_{X} \operatorname{Spec}(\bar{k}((u)))$.) Recall that our construction of $\underline{G}$ over $\breve{\mathcal{O}}\left[u, u^{-1}\right]$ in $₫ 2$ produces an isomorphism between the Iwahori-Weyl groups $\widetilde{W}$ of $G \otimes_{F} \breve{F}$ and $\widetilde{W}^{\prime}$ of $G^{\prime}$. The constructions in 8 . above can also be applied to $G \otimes_{F} \breve{F}$ and $\{\mu\}$ to produce a $W_{0}$-orbit $\Lambda$ in $\mathbb{X}_{\bullet}(T)_{I} \subset \widetilde{W}$ and a subset $\operatorname{Adm}(\mu) \subset \widetilde{W}$. Using this identification, we will view $\Lambda$ and $\operatorname{Adm}(\mu)$ also as, respectively, a $W_{0}^{\prime}$-orbit in $\mathbb{X}_{\bullet}\left(T^{\prime}\right)_{I} \subset \widetilde{W}^{\prime}$, and a subset $\operatorname{Adm}(\mu) \subset \widetilde{W^{\prime}}$.

Theorem 8.3. Suppose that $p \nmid\left|\pi_{1}\left(G_{\mathrm{der}}\right)\right|$. Then we have

$$
\mathcal{A}^{P_{\bar{k}}}(\mu)=\bar{M}_{\mathcal{G}, \mu} \otimes_{k_{E}} \bar{k}
$$

as closed subschemes of $\mathrm{Gr}_{P_{\bar{k}}}$. 
Corollary 8.4. Suppose that $p \nmid\left|\pi_{1}\left(G_{\mathrm{der}}\right)\right|$ and that $x$ is special in $\mathcal{B}\left(G_{\breve{F}}, \breve{F}\right)$. Then the scheme $M_{\mathcal{G}, \mu}$ is Cohen-Macaulay and normal and the special fiber $\bar{M}_{\mathcal{G}, \mu}$ is geometrically irreducible and normal.

Proof. It is enough to show that the geometric special fiber $\bar{M}_{\mathcal{G}, \mu} \otimes_{k_{E}} \bar{k}$ is irreducible. Indeed, then by Theorem 8.1, the special fiber $\bar{M}_{\mathcal{G}, \mu}$ is Cohen-Macaulay and we can conclude that $M_{\mathcal{G}, \mu}$ is also Cohen-Macaulay. Normality follows as above. The irreducibility of $\bar{M}_{\mathcal{G}, \mu} \otimes_{k_{E}} \bar{k}$ follows from Theorem 8.3; Indeed, when $x$ is special, $W^{\mathcal{P}_{x}^{\prime}}=W_{0}^{\prime}$ and $W^{\mathcal{P}_{x}^{\prime}} \backslash \operatorname{Adm}^{\mathcal{P}_{x}^{\prime}}(\mu) / W^{\mathcal{P}_{x}^{\prime}}$ has only one extreme element in the Bruhat order, namely the image of $t_{\mu}$ (cf. [76, Prop. 6.15]).

Remark 8.5. a) Some special cases of Theorems 8.1 and 8.3 were known before (e.g [19], [20] for $\mathrm{GL}_{n}$ and $\mathrm{GSp}_{2 n}$ ). See [51] for a survey of these previous results. A description of the components of the special fiber of local models in terms of the $\mu$-admissible set as in Theorem 8.3 was first suggested by Kottwitz and Rapoport in [38].

b) We conjecture that when $p \nmid\left|\pi_{1}\left(G_{\text {der }}\right)\right|, M_{\mathcal{G}, \mu}$ is always Cohen-Macaulay, even if $x$ is not special. This conjecture has recently been proven in the split case by X. He [28].

Now let us discuss the proof of Theorem 8.3 .

Proof. We can assume that $F=\breve{F}$ and the residue field $k=\bar{k}$ is algebraically closed; then we are trying to show $\mathcal{A}^{P_{k}}(\mu)=\bar{M}_{\mathcal{G}, \mu}$. The proof is divided into two parts. The first part is to exhibit $\mathcal{A}^{P_{k}}(\mu)$ as a closed subscheme of $\bar{M}_{\mathcal{G}, \mu}$. Then we apply the coherence conjecture of Rapoport and the first author ([49]) shown in [76] to deduce the theorem.

Proposition 8.6. The scheme $\mathcal{A}^{P_{k}}(\mu)$ is naturally a closed subscheme of $\bar{M}_{\mathcal{G}, \mu}$.

Proof. Let $\lambda \in \Lambda$, where $\Lambda \subset \mathbb{X}_{\bullet}(T)_{I}$ is the $W_{0}$-orbit in $\mathbb{X}_{\bullet}(T)_{I}$ associated to $\mu$ as before. Let $t_{\lambda} \in \widetilde{W}$ be the corresponding element in the Iwahori-Weyl group. These elements then are extreme elements in $\operatorname{Adm}(\mu)$ under the Bruhat order of $\widetilde{W}$. To prove the lemma, then it is enough to show that $S_{t_{\lambda}}^{\mathcal{P}_{k}} \subset \bar{M}_{\mathcal{G}, \mu}$ for any $t_{\lambda} \in \Lambda$. This turns out to be a direct consequence of the following two lemmas.

Lemma 8.7. The scheme $\bar{M}_{\mathcal{G}, \mu}$ is invariant under the action of $L^{+} P_{k}$ on $\operatorname{Gr}_{P_{k}}$.

Proof. Recall from $\$ 5$.b.4 that $\mathcal{L}^{+} \mathcal{G}$ acts naturally on $\operatorname{Gr}_{\mathcal{G}, X}$. Now let $x: \operatorname{Spec}(\kappa) \rightarrow X$ be a point where $\kappa$ is either the residue field of $\mathcal{O}$ or the fractional field of $\mathcal{O}$. Then by definition $\mathcal{L}^{+} \mathcal{G} \times_{X} \operatorname{Spec}(\kappa) \simeq L^{+} \mathcal{G}_{\kappa, x}$, and the induced action of $\mathcal{L}^{+} \mathcal{G} \times_{X} \operatorname{Spec}(\kappa)$ on $\operatorname{Gr}_{\mathcal{G}, X} \times_{X} \operatorname{Spec}(\kappa)$ is just the local action of $L^{+} \mathcal{G}_{\kappa, x}$ on $\operatorname{Gr}_{\mathcal{G}_{\kappa, x}}$. Let $\left(\mathcal{L}^{+} \mathcal{G}\right)_{\mathcal{O}}:=$ $\mathcal{L}^{+} \mathcal{G} \times{ }_{X} \operatorname{Spec}(\mathcal{O})$ where the section $\operatorname{Spec}(\mathcal{O}) \rightarrow X$ is given by $u \mapsto \varpi$ as before. Then $\left(\mathcal{L}^{+} \mathcal{G}\right)_{\mathcal{O}}$ acts on $\operatorname{Gr}_{\mathcal{G}, \mathcal{O}}$. Over $E$, this is just the action of $\left(L^{+} \mathcal{G}_{F, \varpi}\right)_{E}$ on $\left(\operatorname{Gr}_{\mathcal{G}_{F, \varpi}}\right)_{E}=$ $\operatorname{Gr}_{\mathcal{G}_{F, \varpi}} \times \operatorname{Spec}(F) \operatorname{Spec}(E)$. Since $\bar{M}_{\mathcal{G}, \mu}$ is defined to be the Zariski closure of $\left(L^{+} \mathcal{G}_{F, \varpi}\right)_{E} \cdot\left[s_{\mu}\right]$, the special fiber carries the natural action of $L^{+} P_{k}=L^{+} \mathcal{G}_{k, 0}$.

To state the second Lemma, recall that $\operatorname{Gr}_{\mathcal{G}, \mathcal{O}}$ is ind-proper (Proposition [5.3). For $\lambda \in \Lambda$, let $\tilde{\lambda} \in \bar{W} \mu$ be a lift of $\lambda$. The $E$-point $\left[s_{\tilde{\lambda}}\right]$ of $\operatorname{Gr}_{\mathcal{G}, \mathcal{O}}$ (cf. 6 .a.1) gives rise to a unique $\mathcal{O}_{E}$-section of $\operatorname{Gr}_{\mathcal{G}, \mathcal{O}}$, still denoted by $\left[s_{\tilde{\lambda}}\right]$. Let 0 be the closed point of $\operatorname{Spec}\left(\mathcal{O}_{E}\right)$. 
Lemma 8.8. We have $\left[s_{\tilde{\lambda}}\right](0)=t_{\lambda}$ in $\mathrm{Gr}_{P_{k}}$.

Proof. Let $\mathcal{O}[u] \rightarrow \mathcal{O}[v]$ be given by $u \mapsto v^{e}$. Set

$$
\mathcal{T}=\operatorname{Res}_{\mathcal{O}[v] / \mathcal{O}[u]}\left(T_{H} \otimes \mathcal{O}[v]\right)^{\gamma},
$$

and let $\mathcal{T}^{0}$ be the neutral connected component of $\mathcal{T}$. Then by construction of the group scheme $\mathcal{G}$ in Theorem 3.1, $\mathcal{T}^{0}$ is a subgroup of $\mathcal{G}$. Let $\mathcal{L} \mathcal{T}, \mathcal{L} \mathcal{T}^{0}$ be the global loop groups over $X=\mathbb{A}_{\mathcal{O}}^{1}$, whose definitions are similar to (5.3). Exactly as in the proof of [76, Prop. 3.5] we see that a cocharacter $\nu$ of $G$ defined over $E$ gives rise to a map

$$
s_{\nu, \mathcal{O}_{E}}: \operatorname{Spec}\left(\mathcal{O}_{E}\right) \rightarrow \mathcal{L} \mathcal{T}^{0} \rightarrow \mathcal{L} \mathcal{G}
$$

such that: (i) the restriction of this map to $\operatorname{Spec}(E) \rightarrow \mathcal{L T}^{0} \rightarrow \mathcal{L G}$ is the point $s_{\nu}$ as in 6.a.1 and (ii) $s_{\nu, \mathcal{O}_{E}}(0) \in \mathcal{L T}^{0}(k)=T(k((u)))$ maps to $t_{\nu}$ under the Kottwitz homomorphism $T(k((u))) \rightarrow \mathbb{X}_{\bullet}(T)_{I}$. Clearly, these two statements together imply the lemma.

This now concludes the proof of Proposition 8.6.

Now we proceed with the second part of the proof of the theorem. To apply the coherence conjecture, we need to construct a natural line bundle on $\operatorname{Gr}_{\mathcal{G}, X}$. The construction is parallel to [76, Sect. 4] where we also refer the reader for more information. Let $\mathcal{V}_{0}=$ Lie $\mathcal{G}$ be the Lie algebra of $\mathcal{G}$. By [64, this is a free $\mathcal{O}[u]$-module of $\operatorname{rank} \operatorname{dim}_{F} G$. Then the adjoint representation $\mathrm{Ad}: \mathcal{G} \rightarrow \mathrm{GL}\left(\mathcal{V}_{0}\right)$ gives rise to a morphism

$$
\text { ad }: \operatorname{Gr}_{\mathcal{G}, X} \rightarrow \operatorname{Gr}_{\mathrm{GL}\left(\mathcal{V}_{0}\right), X}
$$

Over $\operatorname{Gr}_{\mathrm{GL}\left(\mathcal{V}_{0}\right), X}$, we have the determinant line bundle $\mathcal{L}_{\text {det }}$, defined as usual (for example, see [76, Sect. 4]). Let $\mathcal{L}=\mathcal{L}_{2 c}=\operatorname{ad}^{*}\left(\mathcal{L}_{\text {det }}\right)$ be the corresponding line bundle on $\operatorname{Gr}_{\mathcal{G}, X}$, which is ample. Let us denote by $\mathcal{L}_{k}$ the restriction of $\mathcal{L}$ to the special fiber $\operatorname{Gr}_{\mathcal{G}, \mathcal{O}} \otimes_{\mathcal{O}} k \simeq$ $\mathrm{Gr}_{P_{k}}$, and let $\mathcal{L}_{\bar{F}}$ be the restriction of $\mathcal{L}$ to the geometric generic fiber $\operatorname{Gr}_{\mathcal{G}, \mathcal{O}} \otimes_{\mathcal{O}} \bar{F} \simeq$ $\mathrm{Gr}_{\mathcal{G}_{F, \pi}} \otimes_{F} \bar{F} \simeq \mathrm{Gr}_{H} \otimes_{F} \bar{F}$.

Let $M_{\mathcal{G}, \mu, \bar{F}}$ be the geometric generic fiber of $M_{\mathcal{G}, \mu}$. Then for $n \gg 0$,

$$
\operatorname{dim}_{\bar{F}} \Gamma\left(M_{\mathcal{G}, \mu, \bar{F}}, \mathcal{L}_{\bar{F}}^{\otimes n}\right)=\operatorname{dim}_{k} \Gamma\left(\bar{M}_{\mathcal{G}, \mu}, \mathcal{L}_{k}^{\otimes n}\right) \geq \operatorname{dim}_{k} \Gamma\left(\mathcal{A}^{P_{k}}(\mu), \mathcal{L}_{k}^{\otimes n}\right),
$$

and the equality holds for $n \gg 0$ if and only if $\mathcal{A}^{P_{k}}(\mu)=\bar{M}_{\mathcal{G}, \mu}$. Now the coherence conjecture of [49] as proved in [76] implies that

$$
\operatorname{dim}_{\bar{F}} \Gamma\left(M_{\mathcal{G}, \mu, \bar{F}}, \mathcal{L}_{\bar{F}}^{\otimes n}\right)=\operatorname{dim}_{k} \Gamma\left(\mathcal{A}^{P_{k}}(\mu), \mathcal{L}_{k}^{\otimes n}\right)
$$

for any $n$ and this is enough to conclude the proof. For clarity, we explain here how to deduce the equality (8.19) from the results of [76]. First we assume that $G_{\text {der }}$ is simply-connected. Then we can write $G_{\text {der }}=\operatorname{Res}_{F_{1} / F} G_{1} \times \cdots \times \operatorname{Res}_{F_{m} / F} G_{m}$ as the decomposition into simple factors, where $G_{i}$ are almost simple, absolutely simple and simply-connected groups defined over $F_{i}$. Let $\left\{\mu_{i}\right\}$ be the corresponding conjugacy classes of cocharacters for $\operatorname{Res}_{F_{i} / F}\left(G_{i}\right)_{\text {ad }}$, where $\left(G_{i}\right)_{\text {ad }}$ is the adjoint group of $G_{i}$, and let $\left(P_{k}\right)_{i}$ be the corresponding parahoric group scheme over $F_{i}$. Then

$$
\mathcal{A}^{P_{k}}(\mu) \simeq \mathcal{A}^{P_{k}}(\mu)^{\circ} \simeq \prod_{i} \mathcal{A}^{\left(P_{k}\right)_{i}}\left(\mu_{i}\right)^{\circ}
$$


and under this isomorphism, $\mathcal{L}_{k} \simeq \mathcal{L}_{1} \otimes \cdots \otimes \mathcal{L}_{m}$, where $\mathcal{L}_{i}$ is ample on $\mathcal{A}^{\left(P_{k}\right)_{i}}\left(\mu_{i}\right)^{\circ}$ with central charge $2 h_{i}^{\vee}$. Here $h_{i}^{\vee}$ is the dual Coxeter number for $G_{i} \otimes_{F_{i}} \bar{F}$, and the central charge is defined in [49, Sect. 10] (also see [76, Sect. 2.2]). On the other hand, $H_{\text {der }}=$ $\prod H_{i}^{m_{i}}$, where $H_{i}$ is the split form for $G_{i}$ and $m_{i}=\left[F_{i}: F\right]$. Then $\mu_{i}=\mu_{i, 1}+\cdots+\mu_{i, m_{i}}$ for $\mu_{i, j}$ dominant coweights of $\left(H_{i}\right)_{\text {ad }}$. As usual, if $\mu$ is a coweight of a split group $M$, we denote $\overline{\mathrm{Gr}}_{M, \mu}$ the closure of the corresponding $L^{+} M$ orbit in $\mathrm{Gr}_{M}$, which is indeed the same as $\mathcal{A}^{M \otimes k[[u]]}(\mu)$. We similarly denote $\mathcal{A}^{M \otimes k[[u]]}(\mu)^{\circ}$ by $\overline{\mathrm{Gr}}_{M, \mu}^{\circ}$. We have

$$
M_{\mathcal{G}, \mu, \bar{F}} \simeq \overline{\mathrm{Gr}}_{H, \mu} \simeq \overline{\mathrm{Gr}}_{H_{\mathrm{ad}}, \mu}^{\circ} \simeq \prod \overline{\mathrm{Gr}}_{\left(H_{i}\right)_{\mathrm{ad}}, \mu_{i, j}}^{\circ}
$$

and under this isomorphism, $\mathcal{L}_{\bar{F}} \simeq \bigotimes_{i}\left(\mathcal{L}_{b, i}^{\otimes 2 h_{i}^{\vee}}\right)^{\bigotimes m_{i}}$, where $\mathcal{L}_{b, i}$ is the ample generator of the Picard group of each connected component of $\operatorname{Gr}_{\left(H_{i}\right)_{\text {ad }}}$ (which is isomorphic to $\operatorname{Gr}_{H_{i}}$ ). Now by [76, Theorem 2, Proposition 6.2], we have

$$
\operatorname{dim}_{k} \Gamma\left(\mathcal{A}^{\left(P_{k}\right)_{i}}\left(\mu_{i}\right)^{\circ}, \mathcal{L}_{i}^{\otimes n}\right)=\prod_{j=1}^{m_{i}} \operatorname{dim}_{\bar{F}} \Gamma\left(\overline{\operatorname{Gr}}_{\left(H_{i}\right)_{\mathrm{ad}}, \mu_{i, j}}^{\circ}, \mathcal{L}_{b, i}^{\otimes 2 n h_{i}^{\vee}}\right) .
$$

Combining this equality with the above gives (8.19) in this case.

Next, we consider the general case when $\operatorname{char}(k) \nmid\left|\pi_{1}\left(G_{\text {der }}\right)\right|$. Let

$$
1 \rightarrow S \rightarrow \tilde{G} \rightarrow G \rightarrow 1
$$

be a $z$-extension of $G$, i.e. $S$ is an induced central torus, and $\tilde{G}_{\text {der }}$ is simply-connected. We can further assume that $\tilde{G}$ is also tamely ramified. Then $\{\mu\}$ can be lifted to a geometric conjugacy class $\{\tilde{\mu}\}$ of $\tilde{G}$. Let us apply our construction to $\tilde{G}$ and the corresponding parahoric group scheme to obtain the global affine Grassmannian $\operatorname{Gr}_{\tilde{\mathcal{G}}, \mathcal{O}}$ over $\mathcal{O}$. We can also define $M_{\tilde{\mathcal{G}}, \tilde{\mu}}$. Observe that over $\bar{F}$, we have the natural map $\operatorname{Gr}_{\tilde{\mathcal{G}}, \bar{F}} \rightarrow \operatorname{Gr}_{\mathcal{G}, \bar{F}}$. Under this map, the line bundle $\mathcal{L}$ on $\operatorname{Gr}_{\mathcal{G}, \bar{F}}$ pulls back to the corresponding line bundle on $\operatorname{Gr}_{\tilde{\mathcal{G}}, \bar{F}}$ since the adjoint representation of $\tilde{G}$ factors through $G$. In addition, $M_{\tilde{\mathcal{G}}, \tilde{\mu}, \bar{F}}$ maps isomorphically to $M_{\mathcal{G}, \mu, \bar{F}}$. Therefore,

$$
\operatorname{dim}_{\bar{F}} \Gamma\left(M_{\mathcal{G}, \mu, \bar{F}}, \mathcal{L}_{\bar{F}}^{\otimes n}\right)=\operatorname{dim}_{\bar{F}} \Gamma\left(M_{\tilde{\mathcal{G}}, \tilde{\mu}, \bar{F}}, \mathcal{L}_{\bar{F}}^{\otimes n}\right) .
$$

Likewise, we have $\operatorname{Gr}_{\tilde{P}_{k}} \rightarrow \operatorname{Gr}_{P_{k}}$ which maps $\mathcal{A}^{\tilde{P}_{k}}(\tilde{\mu})$ isomorphically to $\mathcal{A}^{P_{k}}(\mu)$ (cf. [49, Sect. 6]); the corresponding line bundles $\mathcal{L}_{k}$ respect the pullback under this map. Then

$$
\operatorname{dim}_{k} \Gamma\left(\mathcal{A}^{P_{k}}(\mu), \mathcal{L}_{k}^{\otimes n}\right)=\operatorname{dim}_{k} \Gamma\left(\mathcal{A}^{\tilde{P}_{k}}(\tilde{\mu}), \mathcal{L}_{k}^{\otimes n}\right) .
$$

This allows us to reduce to the previous case.

\section{NeArby CyCles AND the CONJecture of Kottwitz}

In this chapter, we study the sheaves of nearby cycles of the local models. We extend work of Gaitsgory and Haines-Ngô (see Theorem 9.5) and among other results, we show Theorems 0.3 (Kottwitz's conjecture) and 0.4 of the introduction.

\section{9.a. The nearby cycles.}


9.a.1. We begin by briefly recalling some general facts. (For more details, see for example [29], [21].) Let $(S, s, \eta)$ be a Henselian trait, i.e. $S$ is the spectrum of a Henselian discrete valuation ring, $s$ is the closed point of $S$ with residue field $k(s)$, and $\eta$ is the generic point of $S$. For our purposes, we will always assume that $k(s)$ is either finite or algebraically closed. Let $\bar{\eta}$ be a geometric point over $\eta$ and $\bar{S}$ be the normalization of $S$ in $\bar{\eta}$. Let $\bar{s}$ be the closed point of $\bar{S}$. Let $I=\operatorname{ker}(\operatorname{Gal}(k(\bar{\eta}) / k(\eta)) \rightarrow \operatorname{Gal}(k(\bar{s}) / k(s)))$ denote the inertia group.

Let $\ell$ be a prime invertible in $\mathcal{O}_{S}$. For a scheme $X$, separated and of finite type over $s$, there is the natural category $\operatorname{Sh}_{c}\left(X \times{ }_{s} \eta, \overline{\mathbb{Q}}_{\ell}\right)$ whose objects are constructible $\overline{\mathbb{Q}}_{\ell}$-sheaves on $X_{\bar{s}}$, together with continuous actions of $\operatorname{Gal}(k(\bar{\eta}) / k(\eta))$, compatible with the action of $\operatorname{Gal}(k(\bar{\eta}) / k(\eta))$ on $X_{\bar{s}}$ via $\operatorname{Gal}(k(\bar{\eta}) / k(\eta)) \rightarrow \operatorname{Gal}(\bar{s} / s)$ (see [66. XIII, 1.2.4]). The natural functor $\operatorname{Sh}_{c}(X) \rightarrow \operatorname{Sh}_{c}\left(X \times{ }_{s} \eta\right)$ is a full embedding with essential image consisting of objects on which the inertia $I$ acts trivially (SGA 7, XIII, 1.1.3). The "bounded derived" category of $\operatorname{Sh}_{c}\left(X \times_{s} \eta, \overline{\mathbb{Q}}_{\ell}\right)$ is denoted by $\mathrm{D}_{c}^{b}\left(X \times_{s} \eta, \overline{\mathbb{Q}}_{\ell}\right)$. The usual perverse $t$-structure on $\mathrm{D}_{c}^{b}\left(X_{\bar{s}}, \overline{\mathbb{Q}}_{\ell}\right)$ is naturally lifted to $\mathrm{D}_{c}^{b}\left(X \times_{s} \eta, \overline{\mathbb{Q}}_{\ell}\right)$, and we have the corresponding category of perverse sheaves $\operatorname{Perv}\left(X \times_{s} \eta, \overline{\mathbb{Q}}_{\ell}\right)$. The natural functor $\mathrm{D}_{c}^{b}\left(X, \overline{\mathbb{Q}}_{\ell}\right) \rightarrow \mathrm{D}_{c}^{b}\left(X \times_{s} \eta, \overline{\mathbb{Q}}_{\ell}\right)$ is a full embedding, and its essential image consists of objects in $\mathrm{D}_{c}^{b}\left(X \times_{s} \eta, \overline{\mathbb{Q}}_{\ell}\right)$ on which $I$ acts trivially.

Recall that if $p: \mathfrak{X} \rightarrow S$ is a morphism of schemes which is separated and of finite type, then there is the so-called nearby cycle functor

$$
\mathrm{R} \Psi^{\mathfrak{X}}: \mathrm{D}_{c}^{b}\left(\mathfrak{X}_{\eta}, \overline{\mathbb{Q}}_{\ell}\right) \rightarrow \mathrm{D}_{c}^{b}\left(\mathfrak{X}_{s} \times_{s} \eta, \overline{\mathbb{Q}}_{\ell}\right),
$$

which restricts to an exact functor ([29, Sect. 4])

$$
\mathrm{R} \Psi^{\mathfrak{X}}: \operatorname{Perv}\left(\mathfrak{X}_{\eta}, \overline{\mathbb{Q}}_{\ell}\right) \rightarrow \operatorname{Perv}\left(\mathfrak{X}_{s} \times_{s} \eta, \overline{\mathbb{Q}}_{\ell}\right),
$$

Let $f: \mathfrak{X} \rightarrow \mathfrak{Y}$ be a morphism over $S$. There is a canonical natural transform $f_{!} R \Psi^{\mathfrak{X}} \rightarrow$ $R \Psi^{\mathfrak{Y}} f$ ! which is an isomorphism if $f$ is proper. In addition, there is a canonical natural transform $f^{*} R \Psi^{\mathfrak{Y}} \rightarrow R \Psi^{\mathfrak{X}} f^{*}$, which is an isomorphism if $f$ is smooth.

We will also occasionally use the vanishing cycle functor $([66, \mathrm{XII}])$

$$
\mathrm{R} \Phi^{\mathfrak{X}}: \mathrm{D}_{c}^{b}\left(\mathfrak{X}, \overline{\mathbb{Q}}_{\ell}\right) \rightarrow \mathrm{D}_{c}^{b}\left(\mathfrak{X}_{s} \times_{s} \eta, \overline{\mathbb{Q}}_{\ell}\right),
$$

which, roughly speaking, is defined via the distinguished triangle

$$
\mathcal{F}_{\bar{s}} \rightarrow \mathrm{R} \Psi^{\mathfrak{X}}\left(\mathcal{F}_{\eta}\right) \rightarrow \mathrm{R} \Phi^{\mathfrak{X}}(\mathcal{F}) \rightarrow .
$$

A theorem of Gabber (cf. [29, Sect. 4]) says that $\mathrm{R} \Phi^{\mathfrak{X}}[-1]$ is also perverse exact with the $t$-structure on $\mathrm{D}_{c}^{b}\left(\mathfrak{X}, \overline{\mathbb{Q}}_{\ell}\right)$ defined as in loc. cit.

Remark 9.1. As explained in [17, A. 2], if $X$ is an ind-scheme, of ind-finite type over a field, then $\mathrm{D}_{c}^{b}\left(X, \overline{\mathbb{Q}}_{\ell}\right)$ is defined as the direct limit of the corresponding category on finite dimensional closed subschemes. As the push-forward along closed immersions is perverse exact, this allows to also define a corresponding category $\operatorname{Perv}\left(X, \overline{\mathbb{Q}}_{\ell}\right)$. Similarly, we then have $\mathrm{D}_{c}^{b}\left(X \times_{s} \eta, \overline{\mathbb{Q}}_{\ell}\right)$ and $\operatorname{Perv}\left(X \times_{s} \eta, \overline{\mathbb{Q}}_{\ell}\right)$. If $\mathfrak{X}$ is of ind-finite type over $\mathcal{O}$, we can

\footnotetext{
${ }^{9}$ As usual, this category is not the "real" derived category of $\operatorname{Sh}_{c}\left(X \times_{s} \eta, \overline{\mathbb{Q}}_{\ell}\right)$, but is defined via a limit process. See [25, Footnote 2].
} 
also define the nearby cycles $R \Psi^{\mathfrak{x}}$ and the results in the above discussion appropriately extend to this case.

In what follows, without mentioning it explicitly, we will understand that any category of sheaves on an ind-scheme is defined as a direct limit as above.

9.a.2. Let us now return to our set up, so $S=\operatorname{Spec}(\mathcal{O})$, where $\mathcal{O}$ is the ring of integers of a $p$-adic field $F$ with residue field $k$. Let us fix a prime $\ell$, which is invertible in $\mathcal{O}$.

Let $X_{0}=\operatorname{Spec}(\mathcal{O}) \rightarrow X=\mathbb{A}_{\mathcal{O}}^{1}$ be the morphism given by $u \mapsto 0$ and let $P=P_{\mathcal{O}}$ be the group scheme over $\mathcal{O}[[t]]$ given by

$$
P=\mathcal{G} \times_{X} \operatorname{Spec}(\mathcal{O}[[t]]), \quad u \mapsto t .
$$

Then for $\kappa$ either the fraction field $F$ of $\mathcal{O}$, or the residue field $k$ of $\mathcal{O}, P \times_{\mathcal{O}[[t]]} \kappa[[t]]=P_{\kappa}$ is the parahoric group scheme over $\kappa[[t]]$ associated to the point $x_{\kappa((u))}$ in the building of $\mathcal{B}\left(G_{\kappa}, \kappa((u))\right)$ as in $\$ 3$. a , see also 5. b.5. Let us also consider

$$
\operatorname{Gr}_{P}:=\operatorname{Gr}_{\mathcal{G}, \mathcal{O}, 0}=\operatorname{Gr}_{\mathcal{G}, X} \times_{X} X_{0}
$$

given by this specialization along $u=0$. This is identified with the local affine Grassmannian $\operatorname{Gr}_{\mathcal{G}}$ over $\operatorname{Spec}(\mathcal{O})$ considered in 55 .b.1. The jet group $L^{+} P$ over $\mathcal{O}$ is defined as follows: for every $\mathcal{O}$-algebra $R$,

$$
L^{+} P(R)=P(R[[t]]) .
$$

Then $L^{+} P$ acts on $\mathrm{Gr}_{P}$.

Let $\operatorname{Spec}(\kappa) \rightarrow \operatorname{Spec}(\mathcal{O})$ be a perfect field-valued point. Then $\operatorname{Gr}_{P} \times_{\operatorname{Spec}(\mathcal{O})} \operatorname{Spec}(\kappa)=$ $\mathrm{Gr}_{P_{\kappa}}=\operatorname{Gr}_{P, \kappa}$ is the affine Grassmannian associated to $P_{\kappa}$, and when we base change the action of $L^{+} P$ on $\mathrm{Gr}_{P}$ under $\mathcal{O} \rightarrow \kappa$, we obtain the usual action of $L^{+} P_{\kappa}$ on $\mathrm{Gr}_{P_{\kappa}}$.

For simplicity, we set $\breve{P}=P \otimes_{\mathcal{O}} \breve{\mathcal{O}}$, similarly for the other (ind)-schemes. The $L^{+} \breve{P}_{\text {- }}$ orbits of $\mathrm{Gr}_{\breve{P}}$ are parametrized by certain double cosets in the extended Weyl group $\widetilde{W}$. For $w \in \widetilde{W}$, let $\stackrel{\circ}{S}_{w}$ be the corresponding orbit which is smooth over $\breve{\mathcal{O}}$, and let $S_{w}$ be the corresponding Schubert scheme over $\breve{\mathcal{O}}$. Recall that the group splits after an extension of $\breve{\mathcal{O}}$ of degree prime to $p$. We can see that if $\breve{\mathcal{O}} \rightarrow \kappa$ is as above, then there is a nilpotent immersion $S_{w, \kappa} \rightarrow S_{w} \otimes_{\breve{\mathcal{O}}} \kappa$ where $S_{w, \kappa}$ is the Schubert variety $S_{w, \kappa}$ in $\operatorname{Gr}_{P_{\kappa}}$ corresponding to $w$. (This immersion is an isomorphism if $p \nmid\left|\pi_{1}\left(G_{\text {der }}\right)\right|$. Indeed, then the Schubert varieties $S_{w, \kappa}$ are normal and the result follows using [49, Prop. 9.11].) We will often identify $\overline{\mathbb{Q}}_{\ell}$-sheaves on $S_{w} \otimes_{\breve{\mathcal{O}}} \kappa$ with the corresponding $\overline{\mathbb{Q}}_{\ell}$-sheaves on $S_{w, \kappa}$.

We denote $\mathrm{IC}_{w}$ to be the intersection cohomology sheaf on $S_{w}$, i.e., the intermediate extension of $\overline{\mathbb{Q}}_{\ell}\left[\operatorname{dim} S_{w}+1\right]\left(\operatorname{dim} S_{w} / 2\right)$ on $\stackrel{S}{S}_{w}$. (Here $\operatorname{dim} S_{w}$ is the relative dimension over $\breve{\mathcal{O}}$. For the definition of perverse sheaves on schemes over $\mathcal{O}$, we refer to [29, Sect. 4].) If $S_{w}$ is defined over the discrete valuation ring $\mathcal{O}^{\prime}$ with $\mathcal{O} \subset \mathcal{O}^{\prime} \subset \breve{\mathcal{O}}$, we will keep track of the action of $\operatorname{Gal}\left(\mathcal{O}^{\prime} / \mathcal{O}\right)$ on $\mathrm{IC}_{w}$, or equivalently, regard $\mathrm{IC}_{w}$ also defined over $\mathcal{O}^{\prime}$. For a $\kappa$-valued point of $\operatorname{Spec}(\breve{\mathcal{O}})$, the intersection cohomology sheaf on $S_{w, \kappa}$ is denoted by $\mathrm{IC}_{w, \kappa}$.

When $G=H \otimes_{\mathcal{O}} F$ with $H$ a split Chevalley group over $\mathcal{O}$ and $\mathcal{G}=H \times_{\operatorname{Spec}(\mathcal{O})} X$, then $\operatorname{Gr}_{P}$ is the affine Grassmannian $\mathrm{Gr}_{H}$ over $\mathcal{O}$ and $L^{+} P$ is $L^{+} H$. The $L^{+} H$-orbits of $\mathrm{Gr}_{H}$ are parameterized by conjugacy classes of one-parameter subgroups of $H$ and for 
$\mu \in \mathbb{X}_{\bullet}\left(T_{H}\right) \subset \widetilde{W}$, we denote $S_{\mu}$ by $\overline{\mathrm{Gr}}_{\mu}$. Similarly, we denote by $\mathrm{IC}_{\mu}$ the intersection cohomology sheaf on $\overline{\mathrm{Gr}}_{\mu}$.

9.a.3. Let $M_{\mathcal{G}, \mu, E}$ denote the generic fiber of $M_{\mathcal{G}, \mu}$. If $H$ is the split form of $G$, then $M_{\mathcal{G}, \mu, \tilde{F}}=M_{\mathcal{G}, \mu, E} \otimes_{E} \tilde{F}$ is a projective subvariety of $\operatorname{Gr}_{\mathcal{G}, \mathcal{O}} \otimes_{\mathcal{O}} \tilde{F} \simeq \mathrm{Gr}_{H} \otimes_{\mathcal{O}} \tilde{F}$ by Corollary 5.6. In general $M_{\mathcal{G}, \mu, E}$ is not smooth unless $\mu$ is minuscule. We denote $\mathcal{F}_{\mu}$ to be the intersection cohomology sheaf on the generic fiber $M_{\mathcal{G}, \mu, E}$. Then the pull back of $\mathcal{F}_{\mu}$ to $M_{\mathcal{G}, \mu, \tilde{F}}$ is isomorphic to $\mathrm{IC}_{\mu, \tilde{F}}$. Our goal is to establish a commutativity constraint for the nearby cycle

$$
\mathrm{R} \Psi_{\mu}:=\mathrm{R} \Psi^{M_{\mathcal{G}, \mu}}\left(\mathcal{F}_{\mu}\right) .
$$

Recall that we denote by $k_{E}$ the residue field of $\mathcal{O}_{E}$. We first need

Lemma 9.2. The perverse sheaf $\mathrm{R} \Psi_{\mu}$ on the special fiber $\bar{M}_{\mathcal{G}, \mu}=M_{\mathcal{G}, \mu} \otimes_{\mathcal{O}_{E}} k_{E} \subset$ $\mathrm{Gr}_{P, k_{E}}$ admits a natural $L^{+} P_{k_{E}}$-equivariant structure, i.e., $\mathrm{R} \Psi_{\mu}$ admits a $L^{+} P_{k_{E}} \otimes_{k_{E}} \bar{k}$ equivariant structure as perverse sheaves on $\mathrm{Gr}_{P, k_{E}} \otimes_{k_{E}} \bar{k}$, which is compatible with the action of $\operatorname{Gal}(\bar{F} / E)$ in an obvious sense (which will be clear from the proof).

Proof. Let $\mathcal{L}_{n}^{+} \mathcal{G}$ be the $n$-th jet group of $\mathcal{G}$, i.e. the group scheme over $X=\mathbb{A}_{\mathcal{O}}^{1}$, whose $R$-points classify pairs $(y, \beta)$ with $y: \operatorname{Spec}(R) \rightarrow X$ and $\beta \in \mathcal{G}\left(\Gamma_{y, n}\right)$, where $\Gamma_{y, n}$ is the $n$-th nilpotent thickening of $\Gamma_{y}$. In other words,

$$
\mathcal{L}_{n}^{+} \mathcal{G}(R)=\mathcal{G}\left(R[u-y] /(u-y)^{n+1}\right)
$$

(cf. (5.3) ). It is clear that $\mathcal{L}_{n}^{+} \mathcal{G}$ is smooth over $X$ and that the action of $\left(\mathcal{L}^{+} \mathcal{G}\right)_{\mathcal{O}_{E}}:=$ $\mathcal{L}^{+} \mathcal{G} \times_{X} \operatorname{Spec}\left(\mathcal{O}_{E}\right)$ on $M_{\mathcal{G}, \mu}$ factors through the action of $\left(\mathcal{L}_{n}^{+} \mathcal{G}\right)_{\mathcal{O}_{E}}:=\mathcal{L}_{n}^{+} \mathcal{G} \times_{X} \operatorname{Spec}\left(\mathcal{O}_{E}\right)$ for some sufficiently large $n$.

Let $m:\left(\mathcal{L}_{n}^{+} \mathcal{G}\right)_{\mathcal{O}_{E}} \times \mathcal{O}_{E} M_{\mathcal{G}, \mu} \rightarrow M_{\mathcal{G}, \mu}$ be the above action. Let $p:\left(\mathcal{L}_{n}^{+} \mathcal{G}\right)_{\mathcal{O}_{E}} \times \mathcal{O}_{E} M_{\mathcal{G}, \mu} \rightarrow$ $M_{\mathcal{G}, \mu}$ be the natural projection. Then there is a canonical isomorphism $m^{*} \mathcal{F}_{\mu} \stackrel{\sim}{\rightarrow} p^{*} \mathcal{F}_{\mu}$ as sheaves on $\left(\mathcal{L}_{n}^{+} \mathcal{G}\right)_{E} \times_{E} M_{\mathcal{G}, \mu}$ since the intersection cohomology sheaf $\mathcal{F}_{\mu}$ is naturally $\left(\mathcal{L}^{+} \mathcal{G}\right)_{E}$-equivariant. By taking nearby cycles, we have a canonical isomorphism

$$
\mathrm{R} \Psi^{\left(\mathcal{L}_{n}^{+} \mathcal{G}\right)_{\mathcal{O}_{E}} \times_{\mathcal{O}_{E}} M_{\mathcal{G}, \mu}}\left(m^{*} \mathcal{F}_{\mu}\right) \stackrel{\sim}{\rightarrow} \mathrm{R} \Psi^{\left(\mathcal{L}_{n}^{+} \mathcal{G}\right)_{\mathcal{O}_{E}} \times_{\mathcal{O}_{E}} M_{\mathcal{G}, \mu}}\left(p^{*} \mathcal{F}_{\mu}\right),
$$

which is equivariant with respect to the action of $\operatorname{Gal}(k(\bar{\eta}) / k(\eta))$. Since both $m$ and $p$ are smooth morphisms and taking nearby cycles commutes with smooth base change, we have

$$
m^{*} \mathrm{R} \Psi^{M_{\mathcal{G}, \mu}}\left(\mathcal{F}_{\mu}\right) \stackrel{\sim}{\rightarrow} p^{*} \mathrm{R} \Psi^{M_{\mathcal{G}, \mu}}\left(\mathcal{F}_{\mu}\right),
$$

compatible with the action of $\operatorname{Gal}(k(\bar{\eta}) / k(\eta))$. The cocycle condition of this isomorphism follows from the corresponding cocycle condition for $m^{*} \mathcal{F}_{\mu} \stackrel{\sim}{\rightarrow} p^{*} \mathcal{F}_{\mu}$. This then establishes the desired action and the lemma follows.

Definition 9.3. We let $\operatorname{Perv}_{L^{+} P_{k}}\left(\operatorname{Gr}_{P_{k}} \times_{k} F, \overline{\mathbb{Q}}_{\ell}\right)$ be the category whose objects are $(\mathcal{F}, \theta)$, where $\mathcal{F} \in \operatorname{Perv}\left(\operatorname{Gr}_{P_{k}} \times_{k} F, \overline{\mathbb{Q}}_{\ell}\right)$, and $\theta: m^{*} \mathcal{F} \stackrel{\sim}{\rightarrow} p^{*} \mathcal{F}$ is a $L^{+} P_{\bar{k}^{-}}$-equivariant structure on $\mathcal{F}$, which is compatible with the action of $\operatorname{Gal}(\bar{F} / F)$.

By the above Lemma, $\mathrm{R} \Psi_{\mu}=\mathrm{R} \Psi^{M_{\mathcal{G}, \mu}}\left(\mathcal{F}_{\mu}\right)$ is an object of $\operatorname{Perv}_{L^{+} P_{\varkappa}}\left(\operatorname{Gr}_{P_{\varkappa}} \times_{\varkappa} E, \overline{\mathbb{Q}}_{\ell}\right)$ for $\varkappa=k_{E}$. 
9.a.4. Let $w \in \widetilde{W}$. Recall that for a chosen reduced expression $\tilde{w}$ of $w$, there is the Demazure resolution $D_{\tilde{w}} \rightarrow S_{w}$, where $D_{\tilde{w}}$ is smooth proper over $\breve{\mathcal{O}}$, containing $\stackrel{\circ}{S}_{w}$ as a Zariski open subset, and $D_{\tilde{w}} \backslash \stackrel{S}{S}_{w}$ is a divisor with normal crossings relative to $\breve{\mathcal{O}}$.

Lemma 9.4. Let $F \subset F^{\prime} \subset \breve{F}, \mathcal{O}^{\prime}$ be the normalization of $\mathcal{O}$ in $F^{\prime}$, and $k^{\prime}$ be the residue field of $\mathcal{O}^{\prime}$. Write for simplicity $P^{\prime}=P \otimes_{\mathcal{O}} \mathcal{O}^{\prime}$. Assume that $S_{w}$ is defined over $\mathcal{O}^{\prime}$. Let

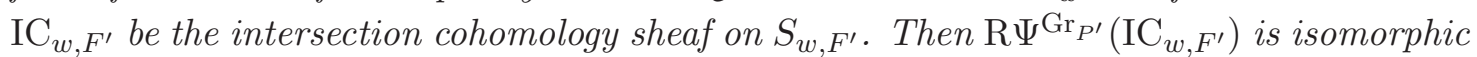
as an object of $\operatorname{Perv}\left(S_{w, k^{\prime}} \times{ }_{k^{\prime}} F^{\prime}, \overline{\mathbb{Q}}_{\ell}\right)$ to the intersection cohomology sheaf $\mathrm{IC}_{w, k^{\prime}}$ of $S_{w, k^{\prime}}$ (recall that we regard $\operatorname{Perv}\left(S_{w, k^{\prime}}, \overline{\mathbb{Q}}_{\ell}\right)$ as a full subcategory of $\operatorname{Perv}\left(S_{w, k^{\prime}} \times k_{k^{\prime}} F^{\prime}, \overline{\mathbb{Q}}_{\ell}\right)$ ).

Proof. The existence of $D_{\tilde{w}}$, together with the argument as in $[25, \S 5.2, \S 6.3]$, implies that the lemma holds for $F^{\prime}=\breve{F}$. Observe that we cannot apply the same argument directly to the case $F^{\prime} \subsetneq \breve{F}$ because $D_{\tilde{w}}$ is not necessarily defined over $\mathcal{O}^{\prime}$. Instead, we argue

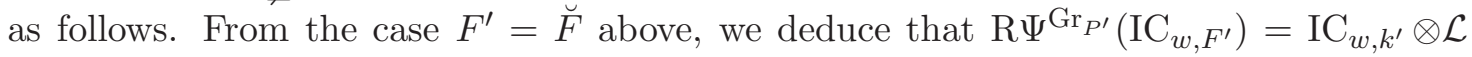
for some rank one local system on $S_{w, k^{\prime}}$ coming from $\operatorname{Spec}\left(k^{\prime}\right)$. On the other hand,

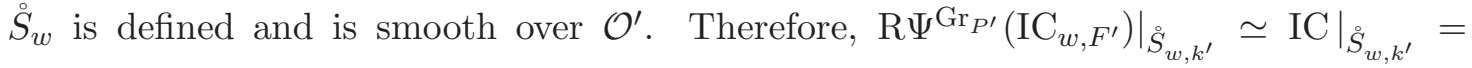
$\overline{\mathbb{Q}}_{\ell}\left[\operatorname{dim} S_{w, k^{\prime}}\right]\left(\left(\operatorname{dim} S_{w, k^{\prime}}\right) / 2\right)$. Hence, $\mathcal{L}$ is trivial.

\section{9.b. A commutativity constraint.}

9.b.1. Let $\mathrm{D}_{L^{+} P}\left(\mathrm{Gr}_{P}, \overline{\mathbb{Q}}_{\ell}\right)$ be the bounded $L^{+} P$-equivariant derived category of (constructible) $\overline{\mathbb{Q}}_{\ell}$-sheaves on $\mathrm{Gr}_{P}$ in the sense of Bernstein-Lunts [6]. Let us recall that $\mathrm{D}_{L+P}\left(\mathrm{Gr}_{P}, \overline{\mathbb{Q}}_{\ell}\right)$ is a monoidal category with structure given by the "convolution product" defined by Lusztig (see [17, 44]). Namely, we have the convolution diagram

$$
\operatorname{Gr}_{P} \times \operatorname{Gr}_{P} \stackrel{q}{\longleftarrow} L P \times \operatorname{Gr}_{P} \stackrel{p}{\longrightarrow} L P \times{ }^{L^{+}} P \operatorname{Gr}_{P}=: \operatorname{Gr}_{P} \tilde{\times} \operatorname{Gr}_{P} \stackrel{m}{\longrightarrow} \operatorname{Gr}_{P}
$$

where $p, q$ are natural projections and $m$ is given by the left multiplication of $L P$ on $\mathrm{Gr}_{P}$. Let $\mathcal{F}_{i} \in \mathrm{D}_{L^{+} P}\left(\operatorname{Gr}_{P}, \overline{\mathbb{Q}}_{l}\right), i=1,2$, and let $\mathcal{F}_{1} \tilde{\times} \mathcal{F}_{2}$ be the unique sheaf (up to a canonical isomorphism) on $L P \times{ }^{L^{+} P} \operatorname{Gr}_{P}$ such that

$$
p^{*}\left(\mathcal{F}_{1} \tilde{\times} \mathcal{F}_{2}\right) \simeq q^{*}\left(\mathcal{F}_{1} \otimes \mathcal{F}_{2}\right) .
$$

Then, by definition

$$
\mathcal{F}_{1} \star \mathcal{F}_{2}=m_{!}\left(\mathcal{F}_{1} \tilde{\times} \mathcal{F}_{2}\right)
$$

If $\operatorname{Spec}(\kappa) \rightarrow \operatorname{Spec}(\mathcal{O})$ is a field valued point, we have the corresponding monoidal category $\mathrm{D}_{L^{+} P_{\kappa}}\left(\operatorname{Gr}_{P_{\kappa}}, \overline{\mathbb{Q}}_{\ell}\right)$ defined in the same manner. Let $\operatorname{Perv}_{L^{+} P_{\kappa}}\left(\operatorname{Gr}_{P_{\kappa}}, \overline{\mathbb{Q}}_{\ell}\right)$ be the core of the perverse $t$-structure.

Observe that if $\mathcal{F}_{1}, \mathcal{F}_{2} \in \operatorname{Perv}_{L^{+} P_{k}}\left(\operatorname{Gr}_{P_{k}} \times_{k} F, \overline{\mathbb{Q}}_{\ell}\right)$, then there is a natural action of $\operatorname{Gal}(\bar{F} / F)$ on $\mathcal{F}_{1} \star \mathcal{F}_{2}$. (In fact, one can define the "derived category" $\mathrm{D}_{L^{+} P_{k}}\left(\operatorname{Gr}_{P_{k}} \times_{k} \eta, \overline{\mathbb{Q}}_{\ell}\right)$ so that $\mathcal{F}_{1} \star \mathcal{F}_{2}$ will be an object in $D_{L^{+} P_{k}}\left(\operatorname{Gr}_{P_{k}} \times_{k} \eta, \overline{\mathbb{Q}}_{\ell}\right)$. We will not use this concept in the paper.) 
9.b.2. In general, if $\mathcal{F}_{1}, \mathcal{F}_{2}$ are perverse sheaves, it is not always the case that $\mathcal{F}_{1} \star \mathcal{F}_{2}$ is perverse. However, the main result of this subsection is

Theorem 9.5. There is a canonical isomorphism

$$
c_{\mathcal{F}}: \mathrm{IC}_{w, \bar{k}} \star \mathrm{R} \Psi_{\mu} \stackrel{\sim}{\rightarrow} \mathrm{R} \Psi_{\mu} \star \mathrm{IC}_{w, \bar{k}}
$$

of perverse sheaves on $\mathrm{Gr}_{P_{\bar{k}}}$. In addition, if $S_{w, \bar{k}}$ is defined over $k^{\prime} \supset k_{E}$, this isomorphism respects the action of $\operatorname{Gal}\left(\bar{F} / E^{\prime}\right)$ on both sides, where $E^{\prime}=E F^{\prime} \subset \bar{F}$, and $F^{\prime}$ is the unique subfield in $\breve{F}$ with residue field $k^{\prime}$.

Remark 9.6. In the case $G=\mathrm{GL}_{n}$ or $\mathrm{GSp}_{2 n}$, and $x$ is in an alcove, i.e the parahoric group is an Iwahori, this is one of the main results of [25] (loc. cit. Proposition 22).

The proof is given by a mixed characteristic version of the arguments in [17, 16]. We need a version of the Beilinson-Drinfeld Grassmannian defined over $X=\mathbb{A}_{\mathcal{O}}^{1}$. For a scheme $y: S \rightarrow X$ we set,

$$
\operatorname{Gr}_{\mathcal{G}, X}^{\mathrm{BD}}(S)=\left\{\begin{array}{l|c}
\mathcal{E} \text { a } \mathcal{G} \text {-torsor on } X \times S, \\
\text { iso-classes of pairs }(\mathcal{E}, \beta) & \begin{array}{c}
\mathcal{B} \text { a trivialization of }\left.\mathcal{E}\right|_{(X \times S) \backslash \Gamma_{y} \cup(0 \times S)}
\end{array}
\end{array}\right\} .
$$

Here $X \times S=X \times_{\operatorname{Spec}(\mathcal{O})} S$. To prove that $\mathrm{Gr}_{\mathcal{G}, X}^{\mathrm{BD}}$ is indeed represented by an ind-scheme, one proceeds as in the proof of Proposition 5.5. Namely, it is standard ([5]) that $\mathrm{Gr}_{\mathrm{GL}_{n}, X}^{\mathrm{BD}}$ is represented by an ind-scheme. The general case follows from the fact that if $\mathcal{G} \rightarrow \mathrm{GL}_{n}$ is a closed embedding such that $\mathrm{GL}_{n} / \mathcal{G}$ is quasi-affine, then $\operatorname{Gr}_{\mathcal{G}, X}^{\mathrm{BD}} \rightarrow \operatorname{Gr}_{\mathrm{GL}_{n}, X}^{\mathrm{BD}}$ is a locally closed embedding.

Let us describe $\operatorname{Gr}_{\mathcal{G}, X}^{\mathrm{BD}}$ more explicitly. For this purpose, set $\stackrel{\circ}{X}=\operatorname{Spec}\left(\mathcal{O}\left[u, u^{-1}\right]\right) \hookrightarrow X$ and let $X_{0}=\operatorname{Spec}(\mathcal{O}) \hookrightarrow X$ be given by $u \mapsto 0$ as before.

The following isomorphisms are clear

$$
\left.\operatorname{Gr}_{\mathcal{G}, X}^{\mathrm{BD}}\right|_{X} \simeq \operatorname{Gr}_{P} \times\left._{\operatorname{Spec}(\mathcal{O})} \operatorname{Gr}_{\mathcal{G}, X}\right|_{X},\left.\quad \operatorname{Gr}_{\mathcal{G}, X}^{\mathrm{BD}}\right|_{X_{0}} \simeq \operatorname{Gr}_{P}
$$

Let us set $\operatorname{Gr}_{\mathcal{G}, \mathcal{O}}^{\mathrm{BD}}:=\operatorname{Gr}_{\mathcal{G}, X}^{\mathrm{BD}} \times_{X} \operatorname{Spec}(\mathcal{O})$, where $\varpi: \operatorname{Spec}(\mathcal{O}) \rightarrow X$ is given by $u \mapsto \varpi$. Observe that on

$$
\operatorname{Gr}_{\mathcal{G}, E^{\prime}}^{\mathrm{BD}}=\operatorname{Gr}_{\mathcal{G}, \mathcal{O}}^{\mathrm{BD}} \times_{\operatorname{Spec}(\mathcal{O})} \operatorname{Spec}\left(E^{\prime}\right) \simeq \operatorname{Gr}_{P_{E^{\prime}}} \times_{\operatorname{Spec}\left(E^{\prime}\right)} \operatorname{Gr}_{\mathcal{G}, E^{\prime}}
$$

we can form $\mathrm{IC}_{w, E^{\prime}} \otimes \mathcal{F}_{\mu}$ over $S_{w, E^{\prime}} \times_{\operatorname{Spec}\left(E^{\prime}\right)} M_{\mathcal{G}, \mu, E^{\prime}}$. (Here and in what follows, for simplicity, we write again $\mathcal{F}_{\mu}$ for the pull-back of $\mathcal{F}_{\mu}$ to the base change over $E^{\prime}$.)

Clearly, Theorem 9.5 is a consequence of the following.

Proposition 9.7. We have canonical isomorphisms in $\operatorname{Perv}_{L^{+} P_{k^{\prime}}}\left(\operatorname{Gr}_{P_{k^{\prime}}} \times_{k^{\prime}} E^{\prime}, \overline{\mathbb{Q}}_{\ell}\right)$ :

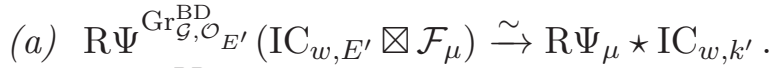

(b) $\mathrm{R} \Psi^{\mathrm{Gr}_{\mathcal{G}, \mathcal{O}_{E^{\prime}}}^{\mathrm{BD}}}\left(\mathrm{IC}_{w, E^{\prime}} \otimes \mathcal{F}_{\mu}\right) \stackrel{\sim}{\rightarrow} \mathrm{IC}_{w, k^{\prime}} \star \mathrm{R} \Psi_{\mu}$.

Proof. In order to simplify the notation, in this proof we will set $\mathcal{O}^{\prime}=\mathcal{O}_{E^{\prime}}$. 
We first prove (a). We define an ind-scheme over $X$ by attaching to every morphism $y: S \rightarrow X$,

$$
\operatorname{Gr}_{\mathcal{G}, X}^{\text {Conv }}(S)=\left\{\begin{array}{l|l}
\text { iso-classes of }\left(\mathcal{E}, \mathcal{E}^{\prime}, \beta, \beta^{\prime}\right) & \begin{array}{c}
\mathcal{E}, \mathcal{E}^{\prime} \text { are two } \mathcal{G} \text {-torsors on } X \times S, \\
\beta \text { a trivialization of }\left.\mathcal{E}\right|_{(X \times S) \backslash \Gamma_{y}}, \\
\beta^{\prime} \text { an isomorphism }\left.\left.\mathcal{E}^{\prime}\right|_{\dot{X} \times S} \simeq \mathcal{E}\right|_{\dot{X} \times S}
\end{array}
\end{array}\right\} .
$$

Observe that there is an natural projection $p: \operatorname{Gr}_{\mathcal{G}, X}^{\text {Conv }} \rightarrow \operatorname{Gr}_{\mathcal{G}, X}$ by forgetting $\left(\mathcal{E}^{\prime}, \beta^{\prime}\right)$, and a natural map $m: \operatorname{Gr}_{\mathcal{G}, X}^{\text {Conv }} \rightarrow \operatorname{Gr}_{\mathcal{G}, X}^{\mathrm{BD}}$ sending $\left(\mathcal{E}, \mathcal{E}^{\prime}, \beta, \beta^{\prime}\right)$ to $\left(\mathcal{E}^{\prime}, \beta \beta^{\prime}\right)$.

The map $p$ makes $\operatorname{Gr}_{\mathcal{G}, X}^{\text {Conv }}$ a fibration over $\operatorname{Gr}_{\mathcal{G}, X}$ with fibers isomorphic to $\operatorname{Gr}_{P}$. To see this, we define a fpqc sheaf $\widetilde{\mathrm{Gr}}_{\mathcal{G}, X}$ over $X$ by setting, for $S$ affine,

$$
\widetilde{\operatorname{Gr}}_{\mathcal{G}, X}(S)=\left\{\begin{array}{l|l}
\text { iso-classes of }\left(\mathcal{E}, \beta, \beta^{\prime}\right) \mid \begin{array}{c}
\mathcal{E} \text { a } \mathcal{G} \text {-torsor on } X \times S, \beta \text { a trivialization of } \\
\left.\mathcal{E}\right|_{(X \times S) \backslash \Gamma_{y}}, \beta^{\prime} \text { a trivialization of }\left.\mathcal{E}\right|_{D_{S}}
\end{array}
\end{array}\right\} .
$$

Then $L^{+} P$ acts on $\widetilde{\operatorname{Gr}}_{\mathcal{G}, X}$ by changing the trivialization $\beta^{\prime}$ and this makes $\widetilde{\operatorname{Gr}}_{\mathcal{G}, X}$ a $L^{+} P$ torsor over $\mathrm{Gr}_{\mathcal{G}, X}$. In addition,

$$
\operatorname{Gr}_{\mathcal{G}, X}^{\text {Conv }}=\widetilde{\operatorname{Gr}}_{\mathcal{G}, X} \times{ }^{L^{+} P} \operatorname{Gr}_{P}
$$

The map $m$ can be described as follows. We have

$$
\left.m\right|_{X}:\left.\left.\mathrm{Gr}_{\mathcal{G}, X}^{\mathrm{Conv}}\right|_{\tilde{X}} \simeq \mathrm{Gr}_{\mathcal{G}, X}^{\mathrm{BD}}\right|_{\tilde{X}},\left.\quad m\right|_{X_{0}}: \mathrm{Gr}_{P} \widetilde{\times} \mathrm{Gr}_{P} \rightarrow \mathrm{Gr}_{P}
$$

where $\operatorname{Gr}_{P} \widetilde{\times} \operatorname{Gr}_{P} \rightarrow \operatorname{Gr}_{P}$ is defined in (9.3).

By specialization along $u \mapsto \varpi$, we obtain corresponding ind-schemes over $\operatorname{Spec}(\mathcal{O})$. Let us further base change all the ind-schemes along $\mathcal{O} \rightarrow \mathcal{O}^{\prime}=\mathcal{O}_{E^{\prime}}$. In particular, we denote $\operatorname{Gr}_{\mathcal{G}, X}^{\text {Conv }} \times{ }_{X} \operatorname{Spec}\left(\mathcal{O}^{\prime}\right)$ by $\operatorname{Gr}_{\mathcal{G}, \mathcal{O}^{\prime}}^{\text {Conv }}$. Similarly, we write $\operatorname{Gr}_{\mathcal{G}, E^{\prime}}^{\text {Conv }}, \widetilde{\operatorname{Gr}}_{\mathcal{G}, E^{\prime}}$, etc. for the base change to $\operatorname{Spec}\left(E^{\prime}\right)$ also given by $u \mapsto \varpi$.

Regard $\mathrm{IC}_{w, E^{\prime}} \otimes \mathcal{F}_{\mu}$ as a sheaf on $\operatorname{Gr}_{\mathcal{G}, E^{\prime}}^{\text {Conv }} \simeq \operatorname{Gr}_{P_{E^{\prime}}} \times_{\operatorname{Spec}\left(E^{\prime}\right)} \operatorname{Gr}_{\mathcal{G}, E^{\prime}}$. Since taking nearby cycles commutes with proper push-forward, to show (a) it will be enough to show that there is a canonical isomorphism

$$
\mathrm{R} \Psi^{\mathrm{Gr} \mathrm{G}_{\mathcal{O}}^{\text {Conv }}}\left(\mathrm{IC}_{w, E^{\prime}} \otimes \mathcal{F}_{\mu}\right) \stackrel{\sim}{\rightarrow} \mathrm{R} \Psi_{\mu} \widetilde{\times} \mathrm{IC}_{w, k^{\prime}}
$$

of sheaves on $\operatorname{Gr}_{P_{k^{\prime}}} \sim \mathrm{Gr}_{P_{k^{\prime}}}$; here $R \Psi_{\mu} \widetilde{\times} \mathrm{IC}_{w, k^{\prime}}$ is the twisted product defined as in (9.3).

Let $L_{n}^{+} P$ be the $n$-th jet group of $P$ whose definition is similar to $\mathcal{L}_{n}^{+} \mathcal{G}$ in (9.1). (In fact, $L_{n}^{+} P=\mathcal{L}_{n}^{+} \mathcal{G} \times_{X} X_{0}$.) Choose $n$ sufficiently large so that the action of $L^{+} P$ on $S_{w}$ factors through $L_{n}^{+} P$. Let $\operatorname{Gr}_{\mathcal{G}, n, X}$ be the $L_{n}^{+} P$-torsor over $\operatorname{Gr}_{\mathcal{G}, X}$ that classifies $\left(\mathcal{E}, \beta, \beta^{\prime}\right)$ where $(\mathcal{E}, \beta)$ are as in the definition of $\operatorname{Gr}_{\mathcal{G}, X}$ and $\beta^{\prime}$ is a trivialization of the restriction of $\mathcal{E}$ over the $n$-th infinitesimal neighborhood $X_{n}$ of $X_{0} \subset X$. Set

$$
\operatorname{Gr}_{\mathcal{G}, n, \mathcal{O}_{E^{\prime}}}=\operatorname{Gr}_{\mathcal{G}, n, X} \times_{X} \operatorname{Spec}\left(\mathcal{O}^{\prime}\right), \quad \operatorname{Gr}_{\mathcal{G}, n, E^{\prime}}=\operatorname{Gr}_{\mathcal{G}, n, X} \times_{X} \operatorname{Spec}\left(E^{\prime}\right) .
$$

Then $\mathcal{F}_{\mu} \widetilde{\times} \mathrm{IC}_{w, E^{\prime}}$ is supported on

$$
\widetilde{\mathrm{Gr}}_{\mathcal{G}, E^{\prime}} \times{ }^{L^{+} P} S_{w} \simeq \mathrm{Gr}_{\mathcal{G}, n, E^{\prime}} \times{ }^{L_{n}^{+} P} S_{w} \subset \mathrm{Gr}_{\mathcal{G}, E^{\prime}}^{\text {Conv }} .
$$

Observe that over $E^{\prime}$, it makes sense to talk about $\mathcal{F}_{\mu} \widetilde{\times} \mathrm{IC}_{w, E^{\prime}}$ (as defined via (9.3)), which is canonically isomorphic to $\mathrm{IC}_{w, E^{\prime}} \otimes \mathcal{F}_{\mu}$. Therefore, (9.5) is equivalent to

$$
\mathrm{R}^{\mathrm{Gr}_{\mathcal{G}, \mathcal{O}^{\prime}}^{\mathrm{Conv}}}\left(\mathcal{F}_{\mu} \widetilde{\times} \mathrm{IC}_{w, E^{\prime}}\right) \simeq \mathrm{R} \Psi_{\mu} \widetilde{\times} \mathrm{IC}_{w, k^{\prime}}
$$


Let us denote the pullback of $\mathcal{F}_{\mu}$ to $\operatorname{Gr}_{\mathcal{G}, n, E^{\prime}}$ by $\widetilde{\mathcal{F}}_{\mu}$. Since $\operatorname{Gr}_{\mathcal{G}, n, X} \rightarrow \operatorname{Gr}_{\mathcal{G}, X}$ is smooth, $\mathrm{R} \Psi^{\mathrm{Gr}_{\mathcal{G}, n, \mathcal{O}^{\prime}}\left(\widetilde{\mathcal{F}}_{\mu}\right)}$ is canonically isomorphic to the pullback of $\mathrm{R} \Psi_{\mu}$, and by Lemma 9.4 .

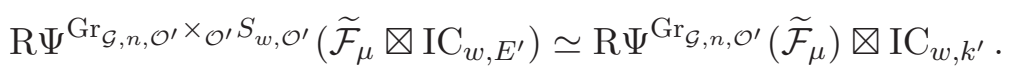

Observe that both sides are in fact $L_{n}^{+} P_{k^{\prime}}$-equivariant perverse sheaves, and the isomorphism respects to the equivariant structure (by the similar argument as in the proof of Lemma 9.2). We thus have (9.6) and therefore have finished the proof of (a).

Next we prove (b), which is similar. There is another convolution affine Grassmannian $\operatorname{Gr}_{\mathcal{G}, X}^{\text {Conv' }}{ }^{\prime}$, which represents the functor that associates every $X$-scheme $y: S \rightarrow X$,

$$
\operatorname{Gr}_{\mathcal{G}, X}^{\text {Conv' }}(S)=\left\{\begin{array}{c|c}
\mathcal{E}, \mathcal{E}^{\prime} \text { are two } \mathcal{G} \text {-torsors on } X \times S, \\
\beta \text { a trivialization of }\left.\mathcal{E}\right|_{X \times S}, \\
\beta^{\prime} \text { an isomorphism }\left.\left.\mathcal{E}^{\prime}\right|_{X \times S \backslash \Gamma_{y}} \simeq \mathcal{E}\right|_{X \times S \backslash \Gamma_{y}}
\end{array}\right\} .
$$

Clearly, we have $m^{\prime}: \operatorname{Gr}_{\mathcal{G}, X}^{\text {Conv }^{\prime}} \rightarrow \operatorname{Gr}_{\mathcal{G}, X}^{\mathrm{BD}}$ by sending $\left(y, \mathcal{E}, \mathcal{E}^{\prime}, \beta, \beta^{\prime}\right)$ to $\left(y, \mathcal{E}^{\prime}, \beta \beta^{\prime}\right)$. This is an isomorphism over $\stackrel{\circ}{X}$, and $\left.m^{\prime}\right|_{X_{0}}$ is again the local convolution diagram

$$
m: \operatorname{Gr}_{P} \widetilde{\times} \operatorname{Gr}_{P} \rightarrow \operatorname{Gr}_{P} .
$$

Again, regard $\mathrm{IC}_{w, E^{\prime}} \otimes \mathcal{F}_{\mu}$ as a sheaf on $\operatorname{Gr}_{\mathcal{G}, E^{\prime}}^{\text {Conv' }} \simeq \operatorname{Gr}_{P_{E^{\prime}}} \times_{E^{\prime}} \operatorname{Gr}_{\mathcal{G}, E^{\prime}}$. Again, as nearby cycles commute with proper push-forward, it is enough to prove that as sheaves on $\operatorname{Gr}_{P_{k^{\prime}}} \widetilde{\times} \operatorname{Gr}_{P_{k^{\prime}}}$,

$$
\mathrm{R} \Psi_{\mathcal{G}, \mathcal{O}^{\prime}}^{\mathrm{Gr}^{\mathrm{Cony}}}\left(\mathrm{IC}_{w, E^{\prime}} \otimes \mathcal{F}_{\mu}\right) \stackrel{\sim}{\rightarrow} \mathrm{IC}_{w, k^{\prime}} \widetilde{\times} R \Psi_{\mu} .
$$

Recall $\mathcal{L}_{n}^{+} \mathcal{G}$ is the $n$-th jet group of $\mathcal{G}$. This is smooth over $X$ and the action of $\left(\mathcal{L}^{+} \mathcal{G}\right)_{\mathcal{O}_{E}}=$ $\mathcal{L}^{+} \mathcal{G} \times_{X} \operatorname{Spec}\left(\mathcal{O}_{E}\right)$ on $M_{\mathcal{G}, \mu}$ factors through $\left(\mathcal{L}_{n}^{+} \mathcal{G}\right)_{\mathcal{O}_{E}}$ for some sufficiently large $n$.

Let us define the $\mathcal{L}_{n}^{+} \mathcal{G}$-torsor $\mathcal{Q}_{n}$ over $\operatorname{Gr}_{P} \times_{\operatorname{Spec}(\mathcal{O})} X$ as follows. Its $S$-points are quadruples $\left(y, \mathcal{E}, \beta, \beta^{\prime}\right)$, where $y: S \rightarrow X,(\mathcal{E}, \beta)$ are as in the definition of $\operatorname{Gr}_{P}$ (and therefore $\beta$ is a trivialization of $\mathcal{E}$ on $X \times S$ ), and $\beta^{\prime}$ is a trivialization of $\mathcal{E}$ over $\Gamma_{y, n}$, the $n$-th nilpotent thickening of the graph $\Gamma_{y}$ of $y$. Then we have the twisted product

$$
\mathcal{T}_{n}:=\left(\mathcal{Q}_{n} \times{ }_{X} \operatorname{Spec}\left(\mathcal{O}^{\prime}\right)\right) \times{ }^{\left(\mathcal{L}_{n}^{+} \mathcal{G}\right)_{\mathcal{O}^{\prime}}} M_{\mathcal{G}, \mu, \mathcal{O}^{\prime}} \subset \operatorname{Gr}_{\mathcal{G}, \mathcal{O}^{\prime}}^{\text {Conv' }}
$$

Over $E^{\prime}$, we can form the twisted product $\mathrm{IC}_{w, E^{\prime}} \widetilde{\times} \mathcal{F}_{\mu}$ on $\mathcal{T}_{n}$ as in (9.3), which is canonically isomorphic to $\mathrm{IC}_{w, E^{\prime}} \otimes \mathcal{F}_{\mu}$. By the same argument as in the proof of (a) (i.e. by pulling back everything to $\left.\left(\mathcal{Q}_{n} \times_{X} \operatorname{Spec}\left(\mathcal{O}^{\prime}\right)\right) \times M_{\mathcal{G}, \mu, \mathcal{O}^{\prime}}\right)$ we have

$$
\mathrm{R} \Psi^{\mathcal{T}_{n}}\left(\mathrm{IC}_{w, E^{\prime}} \widetilde{\times} \mathcal{F}_{\mu}\right) \stackrel{\sim}{\rightarrow} \mathrm{IC}_{w, k^{\prime}} \widetilde{\times} \mathrm{R} \Psi_{\mu} .
$$

Therefore, (9.7) holds and this completes the proof of the proposition.

Remark 9.8. Observe that in [17, the proof of the second statement of the proposition is considerably more difficult than the proof of the first one. Indeed, to prove the second statement, Gaitsgory used the fact that every $H$-torsor over $\mathbb{A} \frac{1}{k}$ admits a reduction to a Borel subgroup, whose counterpart for the general parahoric group schemes $\mathcal{G}$ over $\mathbb{A}_{\mathcal{O}}^{1}$ has not been documented. Instead, our approach exploits the extra flexibility provided by the use of the group schemes $\mathcal{G}$ and treats both cases in a parallel way.

\section{9.c. The monodromy of the nearby cycles.}


9.c.1. Let $M_{\mathcal{G}, \mu}$ be the generalized local model defined as before. This is a flat projective $\mathcal{O}_{E}$-scheme. To study the action of the inertia group $I_{E}=\operatorname{ker}\left(\operatorname{Gal}(\bar{F} / E) \rightarrow \operatorname{Gal}\left(\bar{k} / k_{E}\right)\right)$ ("monodromy") on the nearby cycles, it is convenient to first consider the restriction of this action to the inertia $I_{\tilde{F}}$, where, as we recall (see $\$ 2$.a), $\tilde{F}$ splits the group $G$.

Theorem 9.9. The action of the inertia $I_{\tilde{F}}$ on the nearby cycles $\mathrm{R} \Psi_{\mu}$ is unipotent.

Proof. Consider the base change

$$
\widetilde{M}_{\mathcal{G}, \mu}:=M_{\mathcal{G}, \mu} \otimes \mathcal{O}_{E} \mathcal{O}_{\tilde{F}}
$$

Let us set $\widetilde{\mathrm{R \Psi}}_{\mu}:=\mathrm{R} \Psi^{\widetilde{M}_{\mathcal{G}, \mu}}\left(\mathcal{F}_{\mu}\right)$. Recall that $\widetilde{\mathrm{R} \Psi_{\mu}}$ is canonically isomorphic to the sheaf $\mathrm{R} \Psi_{\mu}$ on $\bar{M}_{\mathcal{G}, \mu} \otimes_{k_{E}} \bar{k}$ but with its $\operatorname{Gal}(\bar{F} / F)$-action restricted to $\operatorname{Gal}(\bar{F} / \tilde{F})$.

To study the inertia action we can base change to $\breve{\mathcal{O}}$. Let $(S, s, \eta)$ be a strictly Henselian trait, i.e. $k(s)$ is separably closed. Suppose that $\mathfrak{X} \rightarrow S$ is a separated scheme of finite type. Let us recall that the nearby cycle $R \Psi^{\mathfrak{X}}$ can be canonically decomposed as

$$
\mathrm{R} \Psi^{\mathfrak{X}}=\left(\mathrm{R} \Psi^{\mathfrak{X}}\right)^{\mathrm{un}} \oplus\left(\mathrm{R} \Psi^{\mathfrak{X}}\right)^{\text {non-un }},
$$

where $\left(\mathrm{R} \Psi^{\mathfrak{X}}\right)^{\text {un }}$ is the unipotent part and $\left(\mathrm{R} \Psi^{\mathfrak{X}}\right)^{\text {non-un }}$ is the non-unipotent part (see [21, $\S 5])$. If $f: \mathfrak{X} \rightarrow \mathfrak{Y}$ is a proper morphism over $S$, then the isomorphism $f_{*} \mathrm{R} \Psi^{\mathfrak{X}} \simeq \mathrm{R} \Psi^{\mathfrak{Y}} f_{*}$ respects this decomposition. We refer to [21] for the details. From this discussion, by using the standard technique of using an Iwahori subgroup contained in our given parahoric, we see that Theorem 9.9 follows from

Proposition 9.10. Theorem 9.9 holds in the case $x \in A(G, A, F)$ lies in the alcove, i.e. when $P_{k}$ is an Iwahori group scheme.

Proposition 9.10 will be deduced as a consequence of the general theory of central sheaves on affine flag varieties together with the following lemma.

Lemma 9.11. Under the above assumptions and notations, the action of $\operatorname{Gal}(\bar{F} / \tilde{F} \breve{F})$ on the cohomology groups $\mathrm{H}^{*}\left(M_{\mathcal{G}, \mu, \tilde{F}},\left(\mathcal{F}_{\mu}\right)_{\tilde{F}}\right)$ is trivial.

Proof. Recall that if $H$ is the split form of $G$, then $M_{\mathcal{G}, \mu, \tilde{F}} \simeq \overline{\mathrm{Gr}}_{H, \mu, \tilde{F}}$ and $\left(\mathcal{F}_{\mu}\right)_{\tilde{F}} \simeq \mathrm{IC}_{\mu, \tilde{F}}$.

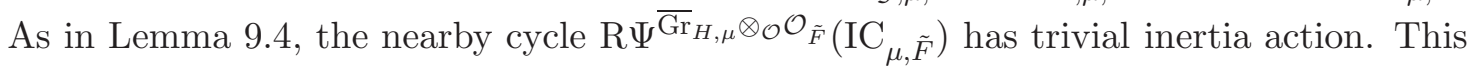
and proper base change implies the lemma.

Now, we prove Proposition 9.10. It is enough to show the statement for $\widetilde{\mathrm{R \Psi}}_{\mu}$. Decompose

$$
\widetilde{\mathrm{R}}_{\mu}=\left(\widetilde{\mathrm{R} \Psi}_{\mu}\right)^{\mathrm{un}} \oplus\left(\widetilde{\mathrm{R}}_{\mu}\right)^{\mathrm{non}-\mathrm{un}}
$$

By taking cohomology and using the above lemma, we obtain $\mathrm{H}^{*}\left(\mathrm{Gr}_{P_{k}},(\widetilde{\mathrm{R \Psi}})^{\text {non-un }}\right)=$ $(0)$. We claim that this already implies that $(\widetilde{\mathrm{R} \Psi})^{\text {non-un }}=(0)$. Indeed, recall that by Theorem 9.5, we have isomorphisms

$$
\widetilde{\mathrm{R} \Psi}_{\mu} \star \mathrm{IC}_{w, \bar{k}} \simeq \mathrm{R} \Psi^{\mathrm{Gr}_{\mathcal{G}, \mathcal{O}_{\tilde{F}}}^{\mathrm{BD}}\left(\mathrm{IC}_{w, E^{\prime}} \otimes \mathcal{F}_{\mu}\right) \simeq \mathrm{IC}_{w, E^{\prime}} \star \widetilde{\mathrm{R} \Psi}}
$$


compatible with the action of the inertia group $\operatorname{Gal}(\bar{F} / \tilde{F} \breve{F})$. Since the inertia action on $\mathrm{IC}_{w, \bar{k}}=\mathrm{R \Psi}^{\mathrm{Gr}_{\breve{P}}}\left(\mathrm{IC}_{w, \breve{F}}\right)$ is trivial, we obtain isomorphisms of perverse sheaves

$$
\begin{aligned}
& \left(\widetilde{\mathrm{R}}_{\mu}\right)^{\text {non }-\mathrm{un}} \star \mathrm{IC}_{w, \bar{k}} \simeq\left(\widetilde{\mathrm{R} \Psi}_{\mu} \star \mathrm{IC}_{w, \bar{k}}\right)^{\text {non }-\mathrm{un}} \\
& \simeq\left(\mathrm{IC}_{w, \bar{k}} \star \widetilde{\mathrm{R} \Psi} \mu\right)^{\text {non-un }} \simeq \mathrm{IC}_{w, \bar{k}} \star(\widetilde{\mathrm{R} \Psi})^{\text {non }-\mathrm{un}} .
\end{aligned}
$$

$([21, \S 5.3])$. In other words, $\left(\widetilde{\mathrm{R \Psi}}_{\mu}\right)^{\text {non-un }}$ is a central sheaf in $\operatorname{Perv}_{L^{+} P_{\bar{k}}}\left(\mathrm{Gr}_{P_{\bar{k}}}, \overline{\mathbb{Q}}_{l}\right)$ (cf. [2, 76]). In loc. cit., it is proven that central sheaves admit filtrations by the so-called Wakimoto sheaves on $\operatorname{Gr}_{P_{\bar{k}}}$. Hence, by [76, Corollary 7.9], $\mathrm{H}^{*}\left(\mathrm{Gr}_{P_{\bar{k}}},\left(\widetilde{\mathrm{R \Psi}}{ }_{\mu}\right)^{\text {non }- \text { un }}\right)=(0)$ implies that $\left(\widetilde{\mathrm{R \Psi}}_{\mu}\right)^{\text {non-un }}=(0)$. This concludes the proof of the Proposition and hence also of Theorem 9.9 .

9.c.2. Recall that we say that $x \in \mathcal{B}(G, F)$ is a very special vertex, if it is special in the sense of Bruhat-Tits in both $\mathcal{B}(G, F)$ and $\mathcal{B}\left(G_{\breve{F}}, \breve{F}\right)$. If there exists such a vertex then the group $G$ is quasi-split over $F$ (see [75, $\S 6]$ ).

Proposition 9.12. Assume that $x$ is a very special vertex. Then the action of the inertia $I_{\tilde{F}}$ on $\mathrm{R} \Psi_{\mu}$ is trivial.

Proof. Observe that when $x$ is a very special vertex, the hypercohomology functor

$$
\mathrm{H}^{*}: \operatorname{Perv}_{L^{+} P_{\bar{k}}}\left(\operatorname{Gr}_{P_{\bar{k}}}, \overline{\mathbb{Q}}_{\ell}\right) \rightarrow \operatorname{Vect}_{\overline{\mathbb{Q}}_{\ell}}
$$

is a faithful functor. This follows from the semisimplicity of $\operatorname{Perv}_{L^{+} P_{\bar{k}}}\left(\operatorname{Gr}_{P_{\bar{k}}}, \overline{\mathbb{Q}}_{\ell}\right)$, as is shown in [45, 75]. Then the proposition is a direct corollary of Lemma 9.11.

Remark 9.13. When $G=\operatorname{Res}_{K / F} \mathrm{GL}_{n}$, where $K$ is a finite extension of $F$ with Galois hull $\tilde{F}$ in $\bar{F}$, and $\mu$ is a Shimura (minuscule) cocharacter, Proposition 9.12 is shown in [47]. When $G$ is a ramified unitary similitude group and $\mu$ is a Shimura cocharacter, it is shown in [75]. In these two cases, one can also obtain an explicit description of the monodromy action of $I_{E}$ on $\mathrm{R} \Psi_{\mu}$. See [47, Sect. 7] (also Example 9.24 below) and [75, Theorem 6.2] respectively. We will return to the subject of the monodromy action on $\mathrm{R} \Psi_{\mu}$ in 9.d.4. There we will give a uniform but less explicit description, in all cases that $G$ is quasi-split and $x$ is very special.

\section{9.d. The semi-simple trace.}

9.d.1. As is explained in [25, §3.1], for any $X$ over $s$, and $\mathbb{F}_{q} \supset k(s)$, there is a map

$$
\tau^{\mathrm{ss}}: \mathrm{D}_{c}^{b}\left(X \times_{s} \eta, \overline{\mathbb{Q}}_{\ell}\right) \rightarrow \operatorname{Func}\left(X\left(\mathbb{F}_{q}\right), \overline{\mathbb{Q}}_{\ell}\right),
$$

called the semi-simple trace. This notion is due to Rapoport. Let us briefly recall its definition and refer to [25] for details.

Let $V$ be an $\ell$-adic representation of the Galois group $\operatorname{Gal}(k(\bar{\eta}) / k(\eta))$. An admissible filtration of $V$ is an increasing filtration $F_{\bullet} V$, stable under the action of $\operatorname{Gal}(k(\bar{\eta}) / k(\eta))$ and such that, for all $i$, the action of the inertia $I$ on $V_{i} / V_{i-1}$ factors through a finite quotient. For an admissible filtration of $V$, one defines

$$
\operatorname{Tr}^{\mathrm{ss}}(\sigma, V)=\sum_{i} \operatorname{Tr}\left(\sigma,\left(\operatorname{gr}_{i}^{F} V\right)^{I}\right),
$$


where $\sigma$ is the geometric Frobenius element. It is well-known that admissible filtrations always exist and that the semi-simple trace $\operatorname{Tr}^{\mathrm{ss}}(\sigma, V)$ does not depend on the choice of admissible filtration.

Next, if $\mathcal{F} \in \mathrm{Sh}_{c}\left(X \times_{s} \eta, \overline{\mathbb{Q}}_{\ell}\right)$, then for every $x \in X\left(\mathbb{F}_{q}\right)$, we define

$$
\tau_{\mathcal{F}}^{\mathrm{ss}}(x)=\operatorname{Tr}^{\mathrm{ss}}\left(\sigma_{x}, \mathcal{F}_{\bar{x}}\right),
$$

where $\bar{x}$ is a geometric point over $x$ and $\sigma_{x}$ is the geometric Frobenius of $\operatorname{Gal}(k(\bar{x}) / k(x))$. In general, if $\mathcal{F} \in \mathrm{D}_{c}^{b}\left(X \times{ }_{s} \eta, \overline{\mathbb{Q}}_{\ell}\right)$, then we set

$$
\tau_{\mathcal{F}}^{\mathrm{sS}}(x)=\sum_{i}(-1)^{i} \tau_{\mathcal{H}^{i} \mathcal{F}}^{\mathrm{ss}}(x) .
$$

It is known that taking the semi-simple trace gives an analogue of Grothendieck's usual sheaf-function dictionary. Namely, we can see that $\tau^{\text {ss }}$ factors through the Grothendieck group of $\mathrm{D}_{c}^{b}\left(X \times{ }_{s} \eta, \overline{\mathbb{Q}}_{\ell}\right)$. Also, if $f: X \rightarrow Y$ is a morphism over $s$, then

$$
f^{*} \tau_{\mathcal{F}}^{\mathrm{ss}}=\tau_{f^{*} \mathcal{F}}^{\mathrm{ss}}, \quad f_{!} \tau_{\mathcal{F}}^{\mathrm{ss}}=\tau_{f_{!} \mathcal{F}}^{\mathrm{ss}} .
$$

9.d.2. Now, consider $x \in \mathcal{B}(G, F)$ and let $\mathcal{P}_{x}$ be the parahoric group scheme as before. Let $G^{\prime}=\mathcal{G} \times{ }_{X} \operatorname{Spec}(k((u)))$ be the corresponding reductive group over $k((u))$ and suppose $\mathcal{P}_{x_{k((u))}}=\mathcal{G} \times_{X} \operatorname{Spec}(k[[u]])$ is a corresponding parahoric group scheme over $k[[u]]$. Let $\mathbb{F}_{q} \supset k$ and set $P_{q}^{\prime}:=\mathcal{P}_{x_{k((u))}}\left(\mathbb{F}_{q}[[u]]\right)$. Let $\mathcal{H}_{q}\left(G^{\prime}, P^{\prime}\right):=\mathcal{H}\left(G^{\prime}\left(\mathbb{F}_{q}((u))\right), P_{q}^{\prime}\right)$ be the Hecke algebra of bi- $P_{q}^{\prime}$-invariant, compactly supported locally constant $\overline{\mathbb{Q}}_{l}$-valued functions on $G^{\prime}\left(\mathbb{F}_{q}((u))\right)$ under convolution. This is an associative algebra (usually non-commutative). Let $\mathcal{Z}\left(\mathcal{H}_{q}\left(G^{\prime}, P^{\prime}\right)\right)$ denote its center.

Since $\mathrm{R} \Psi_{\mu}$ is an object in $\operatorname{Perv}_{L^{+} P_{k_{E}}}\left(\operatorname{Gr}_{P_{k}} \times_{k_{E}} E, \overline{\mathbb{Q}}_{\ell}\right)$, for every $\mathbb{F}_{q} \supset k_{E}$, we can consider $\tau_{\mathrm{R} \Psi_{\mu}}^{\mathrm{Ss}} \in \mathcal{H}_{q}\left(G^{\prime}, P^{\prime}\right)$. As in [25], we can see that Theorem 9.5 implies

Theorem 9.14. With the above assumptions and notations, $\tau_{\mathrm{R} \Psi_{\mu}}^{\mathrm{sS}} \in \mathcal{Z}\left(\mathcal{H}_{q}\left(G^{\prime}, P^{\prime}\right)\right)$.

In what follows, we explain how, in some cases, we can determine (or characterize) the central function $\tau_{\mathrm{R} \Psi_{\mu}}^{\mathrm{SS}}$.

9.d.3. Unramified groups. Here, we assume that $G$ is unramified. This means that the group $G$ is quasi-split and it splits over $\breve{F}$.

a) First assume that $x$ is hyperspecial. Then $\operatorname{Gr}_{P_{\bar{k}}}$ is isomorphic to the usual affine Grassmannian $\operatorname{Gr}_{H} \otimes \bar{k}$ of $H$. In this case, the geometric fiber $\bar{M}_{\mathcal{G}_{, \mu}} \otimes_{k_{E}} \bar{k}$ is isomorphic (up to nilpotents) to the Schubert variety $\overline{\mathrm{Gr}}_{\mu, \bar{k}} \subset \mathrm{Gr}_{H} \otimes \bar{k}$ corresponding to $\mu$. (If $p \nmid\left|\pi_{1}\left(G_{\text {der }}\right)\right|$, these are isomorphic by Theorem 8.3.) Let us denote the intersection cohomology sheaf on $\bar{M}_{\mathcal{G}, \mu}$ by $\overline{\mathcal{F}}_{\mu}$ so that $\left(\overline{\mathcal{F}}_{\mu}\right)_{\bar{k}} \simeq \mathrm{IC}_{\mu, \bar{k}}$. By Proposition 9.12, we know that $\mathrm{R} \Psi_{\mu}$ is a Weil sheaf on $\overline{\mathrm{Gr}}_{\mu, \bar{k}}$. By Lemma 8.7 and Lemma 8.8, $\left(\overline{\mathcal{F}}_{\mu}\right)_{\bar{k}}$ is a subquotient of $\left(\mathrm{R} \Psi_{\mu}\right)_{\bar{k}}$ as Weil sheaves and when forgetting the Weil structure over $k_{E}$, it is a direct summand since $\operatorname{Perv}_{L^{+} P_{\bar{k}}}\left(\operatorname{Gr}_{P_{\bar{k}}}, \overline{\mathbb{Q}}_{\ell}\right)$ is a semi-simple category. Then it follows from

$$
\operatorname{dim}_{\overline{\mathbb{Q}}_{\ell}} \mathrm{H}^{*}\left(\mathrm{IC}_{\mu}\right)=\operatorname{dim}_{\overline{\mathbb{Q}}_{\ell}} \mathrm{H}^{*}\left(\mathcal{F}_{\mu}\right)=\operatorname{dim}_{\overline{\mathbb{Q}}_{\ell}} \mathrm{H}^{*}\left(\mathrm{R} \Psi_{\mu}\right),
$$

that we have

Proposition 9.15. $\mathrm{R} \Psi_{\mu} \simeq \overline{\mathcal{F}}_{\mu}$ as Weil sheaves. 
If $\mu$ is a minuscule coweight and $x$ is hyperspecial, then $\bar{M}_{\mathcal{G}, \mu}$ is smooth. If we set $d=\operatorname{dim} \bar{M}_{\mathcal{G}, \mu}=2(\rho, \mu)$ then, in particular, we have

$$
\mathrm{R} \Psi_{\mu} \simeq \overline{\mathbb{Q}}_{\ell}[d](d / 2)
$$

(the constant sheaf on $\bar{M}_{\mathcal{G}, \mu}$ up to cohomological shift and Tate twist).

For simplicity, write $K=P_{q}^{\prime}$ and denote $\mathcal{H}_{q}\left(G^{\prime}, P^{\prime}\right)$ by $\operatorname{Sph}_{q}$ (the notation stands for the spherical Hecke algebra) which is then already a commutative algebra. It is wellknown that the function

$$
A_{\mu}:=\tau_{\mathcal{F}_{\mu}}^{\mathrm{ss}} \in \operatorname{Sph}_{q}
$$

is given by the Lusztig-Kato polynomial (at least when $G$ is split). Of course, in the case that $\mu$ is minuscule, $A_{\mu}=(-1)^{2(\rho, \mu)} q^{(\rho, \mu)} 1_{K s_{\mu} K}$, where $1_{K s_{\mu} K}$ is the characteristic function of the double coset $K s_{\mu} K$.

b) Next we let $x$ be a general point in $\mathcal{B}(G, F)$. We can then obtain the following statement which was conjectured by Kottwitz and previously proven in the cases $G=\mathrm{GL}_{n}$ and $\mathrm{GSp}_{2 n}$ by Haines and Ngô ([25], see also [59]).

Theorem 9.16. (Kottwitz's conjecture) Assume that $G$ is unramified. Then with the notations above, $\tau_{\mathrm{R} \Psi_{\mu}}^{\mathrm{ss}}$ is the unique element in the center $\mathcal{Z}\left(\mathcal{H}_{q}\left(G^{\prime}, P^{\prime}\right)\right)$, whose image under the Bernstein isomorphism $\mathcal{Z}\left(\mathcal{H}_{q}\left(G^{\prime}, P^{\prime}\right)\right) \stackrel{\sim}{\rightarrow} \operatorname{Sph}_{q}$ is $A_{\mu}$.

Proof. See [24, Theorem 3.1.1] for the Bernstein isomorphism in this case. We first show the result when $x$ is in an alcove, i.e the parahoric subgroup is an Iwahori. Then the proof follows, exactly as is explained in [25], from Theorem 9.14 and Proposition 9.15, by using (9.8) and the fact that taking nearby cycles commutes with proper push-forward. We refer the reader to loc. cit. for more details. The general parahoric case now follows similarly by first finding an Iwahori subgroup contained in the parahoric and then using the compatibility of the Bernstein isomorphism with change in parahoric subgroup ([24, 3.3.1]).

Remark 9.17. Assume that $x$ lies in an alcove, i.e the parahoric subgroup is an Iwahori, and that $\mu$ is minuscule. Using Bernstein's presentation of the Iwahori-Hecke algebras (again at least for $G$ split), it is possible to give explicit formulas for the functions $\tau_{R \Psi_{\mu}}^{\mathrm{Ss}}$. See [25] and 22] for such formulas and various other related results.

9.d.4. Quasi-split groups; the special vertex case. Now, we assume that $G$ is quasi-split (but can be split only after a ramified extension). We will restrict here to the case that $x$ is a very special vertex in $\mathcal{B}(G, F)$, i.e it is special and remains special in $\mathcal{B}\left(G_{\breve{F}}, \breve{F}\right)$ in the sense of Bruhat-Tits. As in this case the special fiber $\bar{M}_{\mathcal{G}, \mu}$ is not necessarily smooth, the nearby cycle $R \Psi_{\mu}$ can be complicated. Our goal is to determine the semi-simple trace $\tau_{\mathrm{R} \Psi_{\mu}}^{\mathrm{ss}}$ but before that we need to understand the monodromy action on $\mathrm{R} \Psi_{\mu}$.

We will start by describing $\widetilde{\mathrm{R} \Psi}_{\mu}$ explicitly. By Proposition $9.12, \widetilde{\mathrm{R}}_{\mu}$ is a Weil sheaf on $\operatorname{Gr}_{P_{\bar{k}}}$.

Let us briefly recall the (ramified) geometric Satake correspondence as established in [45. 75. Let $H^{\vee}$ be the dual group of the split form $H$ of $G$ over $\overline{\mathbb{Q}}_{\ell}$ in the sense of 
Langlands (i.e. the root datum of $H^{\vee}$ is dual to the root datum of $H$ ). Then the Galois $\operatorname{group} \Gamma=\operatorname{Gal}(\tilde{F} / F)$ (and therefore $\left.I=I_{F}\right)$ acts on $H^{\vee}$ via pinned automorphisms (after choosing some pinning). Recall the notation $G^{\prime}=\mathcal{G} \times{ }_{X} \operatorname{Spec}(k((u)))$ and $P_{k}=$ $\mathcal{G} \times_{X} \operatorname{Spec}(k[[u]])$; our constructions allow us to identify the actions of $I_{F}$ and $I_{k((u))}$ on $H^{\vee}$ obtained from $G$ and $G^{\prime}$ respectively: Indeed, the action of $I_{F}$ (resp. $\left.I_{k((u))}\right)$ on $H^{\vee}$ is determined by $I_{F} \rightarrow \operatorname{Out}(G)$ (resp. $\left.I_{k((u))} \rightarrow \operatorname{Out}\left(G^{\prime}\right)\right)$. These homomorphisms factor through the corresponding tame inertia quotients which as in $\$ 2 . b$ are identified with the fundamental group of $\breve{\mathcal{O}}\left[u^{ \pm 1}\right]$. However, by our construction, $\operatorname{Out}(G)=\operatorname{Out}(\underline{G})=$ $\operatorname{Out}\left(G^{\prime}\right)$, and both $I_{F} \rightarrow \operatorname{Out}(G)$ and $I_{k((u))} \rightarrow \operatorname{Out}\left(G^{\prime}\right)$ are obtained as specializations of $\pi_{1}\left(\operatorname{Spec}\left(\breve{\mathcal{O}}\left[u^{ \pm 1}\right]\right), \operatorname{Spec}(\bar{F})\right) \rightarrow \operatorname{Out}(\underline{G})$.

The geometric Satake correspondence of [45, 75] is an equivalence of tensor categories

$$
\mathcal{S}_{\bar{k}}: \operatorname{Rep}\left(\left(H^{\vee}\right)^{I}\right) \stackrel{\sim}{\rightarrow} \operatorname{Perv}_{L^{+} P_{\bar{k}}}\left(\operatorname{Gr}_{P_{\bar{k}}}, \overline{\mathbb{Q}}_{\ell}\right)
$$

such that the composition $\mathrm{H}^{*} \circ \mathcal{S}_{\bar{k}}$ is isomorphic to the natural forgetful fiber functor $\operatorname{Rep}\left(\left(H^{\vee}\right)^{I}\right) \rightarrow \operatorname{Vect}\left(\overline{\mathbb{Q}}_{l}\right)$. On the other hand, $\operatorname{Gr}_{\mathcal{G}, \mathcal{O}} \otimes_{\mathcal{O}} \tilde{F}$ is just $\operatorname{Gr}_{H, \tilde{F}}$ for the split group $H$ and by [45] there is a full embedding

$$
\mathcal{S}_{\tilde{F}}: \operatorname{Rep}\left(H^{\vee}\right) \rightarrow \operatorname{Perv}_{\left(\mathcal{L}^{+} \mathcal{G}\right)_{\tilde{F}}}\left(\operatorname{Gr}_{\mathcal{G}, \mathcal{O}} \otimes_{\mathcal{O}} \tilde{F}, \overline{\mathbb{Q}}_{\ell}\right) .
$$

This maps $V_{\mu}$, the highest weight representation of $H^{\vee}$ corresponding to $\mu \in \mathbb{X} \bullet(T)$, to $\mathrm{IC}_{\mu, \tilde{F}}$, so that $\mathcal{S}_{\tilde{F}}\left(V_{\mu}\right)=\mathrm{IC}_{\mu, \tilde{F}}$. Again, the composition $\mathrm{H}^{*} \circ \mathcal{S}_{\tilde{F}}$ is isomorphic to the forgetful functor.

Theorem 9.18. The composition

$$
\begin{aligned}
& \left(\mathcal{S}_{\bar{k}}\right)^{-1} \circ \mathrm{R \Psi}^{\mathrm{Gr}_{\mathcal{G}, \mathcal{O}} \otimes \mathcal{O}_{\tilde{F}} \circ \mathcal{S}_{\tilde{F}}:} \\
& \quad \operatorname{Rep}\left(H^{\vee}\right) \rightarrow \operatorname{Perv}_{\left(\mathcal{L}^{+} \mathcal{G}\right)_{\tilde{F}}}\left(\operatorname{Gr}_{\mathcal{G}, \mathcal{O}} \otimes_{\mathcal{O}} \tilde{F}, \overline{\mathbb{Q}}_{\ell}\right) \rightarrow \operatorname{Perv}_{L^{+} P_{\bar{k}}}\left(\operatorname{Gr}_{P_{\bar{k}}}, \overline{\mathbb{Q}}_{\ell}\right) \rightarrow \operatorname{Rep}\left(\left(H^{\vee}\right)^{I}\right)
\end{aligned}
$$

is isomorphic to the natural restriction functor

$$
\operatorname{Res}: \operatorname{Rep}\left(H^{\vee}\right) \rightarrow \operatorname{Rep}\left(\left(H^{\vee}\right)^{I}\right) \text {. }
$$

In particular, $\widetilde{\mathrm{R \Psi}}_{\mu} \simeq \mathcal{S}_{\bar{k}}\left(\operatorname{Res}\left(V_{\mu}\right)\right)$.

Proof. Since this is a statement about geometric sheaves, we can assume that the residue field of $\tilde{F}$ is algebraically closed (i.e. we replace $\tilde{F}$ by $\tilde{F} \breve{F}$ in $\bar{F}$ ). In addition, we can ignore the Tate twist in what follows.

Consider the ramified cover $\mathbb{A}_{\mathcal{O}_{\tilde{F}}}^{1} \rightarrow \mathbb{A}_{\mathcal{O}}^{1}$ given by $\mathcal{O}[u] \rightarrow \mathcal{O}_{\tilde{F}}[v], u \mapsto v^{e}$. Let us denote the base change

$$
\widetilde{\operatorname{Gr}}_{\mathcal{G}, X}:=\operatorname{Gr}_{\mathcal{G}, X} \times \mathbb{A}_{\mathcal{O}}^{1} \mathbb{A}_{\mathcal{O}_{\tilde{F}}}^{1} .
$$

We will consider the sub ind-schemes of $\widetilde{\mathrm{Gr}}_{\mathcal{G}, X}$ given by specializing $\mathcal{O}_{\tilde{F}}[v]$ along different directions.

(a) The map $\mathcal{O}_{\tilde{F}}[v] \rightarrow \tilde{F}, v \mapsto \tilde{\varpi}$ gives rise to a closed embedding $i_{v=\tilde{\omega}}: \operatorname{Gr}_{\mathcal{G}, \mathcal{O}} \otimes \mathcal{O}_{\tilde{F}} \rightarrow$ $\widetilde{\mathrm{Gr}}_{\mathcal{G}, X}$. We denote the open embedding $\operatorname{Gr}_{\mathcal{G}, \mathcal{O}} \otimes \tilde{F} \rightarrow \operatorname{Gr}_{\mathcal{G}, \mathcal{O}} \otimes \mathcal{O}_{\tilde{F}}$ by $j_{v=\tilde{\varpi}}$.

(b) The map $\mathcal{O}_{\tilde{F}}[v] \rightarrow \tilde{F}[v]$ gives rise to $j_{\tilde{\varpi} \neq 0}:{\widetilde{\operatorname{Gr}_{\mathcal{G}, \mathbb{A}}}}_{\tilde{F}} \rightarrow{\widetilde{\operatorname{Gr}_{\mathcal{G}}, X}}$. We denote $\widetilde{\mathrm{Gr}}_{\mathcal{G}, \mathbb{G}_{m \tilde{F}}} \rightarrow \widetilde{\mathrm{Gr}}_{\mathcal{G}, \mathbb{A}_{\tilde{F}}^{1}}$ by $j_{\tilde{F}}$. 
(c) The map $\mathcal{O}_{\tilde{F}}[v] \rightarrow \bar{k}[v], \tilde{\varpi} \mapsto 0$ gives rise to $i_{\tilde{\varpi}=0}: \widetilde{\mathrm{Gr}}_{\mathcal{G}, \mathbb{A}_{\bar{k}}^{1}} \rightarrow \widetilde{\operatorname{Gr}}_{\mathcal{G}, X}$. By its

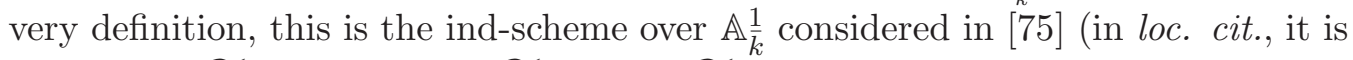
denoted by $\left.\widetilde{\mathrm{Gr}}_{\mathcal{G}}\right)$. We denote $\widetilde{\mathrm{Gr}}_{\mathcal{G}, \mathbb{G}_{m \bar{k}}} \rightarrow{\widetilde{\mathrm{Gr}_{\mathcal{G}, \mathbb{A}} \frac{1}{\bar{k}}}}$ by $j_{\bar{k}}$.

(d) The map $\mathcal{O}_{\tilde{F}}[v] \rightarrow \mathcal{O}_{\tilde{F}}, v \mapsto 0$, gives rise to $i_{v=0}: \operatorname{Gr}_{P_{\mathcal{O}_{\tilde{F}}}} \rightarrow{\widetilde{\operatorname{Gr}_{\mathcal{G}, X}}}_{\text {, and its open }}$, a

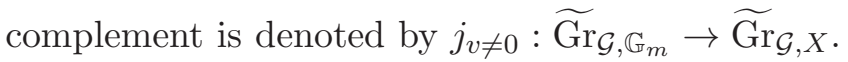

(e) The map $\mathcal{O}_{\tilde{F}}[v] \rightarrow \bar{k}, v \mapsto 0, \varpi \mapsto 0$ gives rise to $i: \operatorname{Gr}_{P_{\bar{k}}} \rightarrow \widetilde{\operatorname{Gr}}_{\mathcal{G}, X}$.

Since the reductive group scheme $\underline{G}$ constructed in Sect. 2 splits after the base change $\mathcal{O}\left[u^{ \pm 1}\right] \rightarrow \mathcal{O}_{\tilde{F}}\left[v^{ \pm 1}\right]$, we have

$$
\widetilde{\operatorname{Gr}}_{\mathcal{G}, \mathbb{G}_{m}} \simeq\left(\mathrm{Gr}_{H} \times \mathbb{G}_{m}\right) \otimes_{\mathcal{O}} \mathcal{O}_{\tilde{F}}
$$

Then for $\mu \in \mathbb{X}_{\bullet}(T)$, we can regard $\mathrm{IC}_{\mu} \otimes \overline{\mathbb{Q}}_{\ell}[1]$, which is a perverse sheaf on $\mathrm{Gr}_{H} \times \mathbb{G}_{m}$, as a perverse sheaf on $\widetilde{\operatorname{Gr}}_{\mathcal{G}, \mathbb{G}_{m}}$. Similarly, we have $\operatorname{IC}_{\mu, \kappa} \otimes \overline{\mathbb{Q}}_{\ell}[1]$ over $\widetilde{\operatorname{Gr}}_{\mathcal{G}, \mathbb{G}_{m \kappa}}$ for $\kappa=\bar{k}$ or $\tilde{F}$.

By the construction of $\mathcal{S}_{\bar{k}}$ in 75 , there is a canonical isomorphism

$$
\mathrm{R} \Psi^{\widetilde{\mathrm{Gr}}_{\mathcal{G}, \mathbb{1} \frac{1}{\bar{k}}}}\left(\mathrm{IC}_{\mu, \bar{k}} \otimes \overline{\mathbb{Q}}_{\ell}[1]\right) \simeq \mathcal{S}_{\bar{k}}\left(\operatorname{Res} V_{\mu}\right)
$$

Therefore, the theorem will follow if we can construct a canonical isomorphism

$$
\mathrm{R} \Psi^{\mathrm{Gr}_{\mathcal{G}, \mathcal{O}} \otimes \mathcal{O}_{\tilde{F}}}\left(\mathcal{S}_{\tilde{F}}\left(V_{\mu}\right)\right) \simeq \mathrm{R} \Psi^{\widetilde{G r}_{\mathcal{G}, \mathbb{A}} \frac{1}{k}}\left(\mathrm{IC}_{\mu, \bar{k}} \otimes \overline{\mathbb{Q}}_{\ell}[1]\right)
$$

In what follows, we will make use of the following standard lemma. (See also [75, Corollary 2.3]).

Lemma 9.19. Let $p: \mathfrak{X} \rightarrow S$ be as in 9.a.1 with $S$ a strictly henselian trait. Let $\mathcal{F}$ be a perverse sheaf on $\mathfrak{X}_{\eta}$. If the inertia action on $\mathrm{R} \Psi^{\mathfrak{X}}(\mathcal{F})$ is trivial, then

$$
\mathrm{R} \Psi^{\mathfrak{X}}(\mathcal{F}) \simeq{ }^{p} \mathrm{H}^{0} i^{*} j_{*} \mathcal{F} \simeq{ }^{p} \mathrm{H}^{1} i^{*} j_{*} \mathcal{F} \simeq i^{*} j_{! *} \mathcal{F},
$$

where ${ }^{p} \mathrm{H}^{*}$ stands for perverse cohomology.

Proof. Here, as usual, we denote by $i$ (resp. $j$ ) the natural closed (resp. open) immersion of the closed (resp. generic) fiber of $p: \mathfrak{X} \rightarrow S$ into $\mathfrak{X}$. Since the inertia action is trivial, from the distinguished triangle

$$
i^{*} j_{*} \mathcal{F} \rightarrow \mathrm{R} \Psi^{\mathfrak{X}}(\mathcal{F}) \stackrel{0}{\rightarrow} \mathrm{R} \Psi^{\mathfrak{X}}(\mathcal{F}) \rightarrow
$$

(see for example [29, (3.6.2)]) we obtain that $i^{*} j_{*} \mathcal{F}$ is supported in perverse cohomological degree 0 and 1 , and both cohomology sheaves are isomorphic to $\operatorname{R} \Psi^{\mathfrak{X}}(\mathcal{F})$. But $i^{*} j_{!_{*}} \mathcal{F}=$ ${ }^{p} \mathrm{H}^{1} i^{*} j_{*} \mathcal{F}$. The lemma follows.

Using this we see that we can reduce (9.10) to proving an isomorphism

$$
{ }^{p} \mathrm{H}^{0} i^{*} j_{\bar{k} *}\left(\mathrm{IC}_{\mu, \bar{k}} \otimes \overline{\mathbb{Q}}_{\ell}\right) \stackrel{\sim}{\rightarrow}{ }^{p} \mathrm{H}^{0} i^{*} j_{v=\tilde{\varpi} *} \mathcal{S}_{\tilde{F}}\left(V_{\mu}\right) .
$$

Observe that there is a natural map $i_{v=\tilde{\varpi}}^{*} j_{v \neq 0 *}\left(\operatorname{IC}_{\mu} \otimes \overline{\mathbb{Q}}_{\ell}\right)[-1] \rightarrow j_{v=\tilde{\varpi} *} \mathcal{S}_{\tilde{F}}\left(V_{\mu}\right)$, which is the adjunction of $j_{v=\tilde{w}}^{*} i_{v=\tilde{w}}^{*} j_{v \neq 0 *}\left(\mathrm{IC}_{\mu} \otimes \overline{\mathbb{Q}}_{\ell}\right)[-1] \stackrel{\sim}{\rightarrow} \mathcal{S}_{\tilde{F}}\left(V_{\mu}\right)$. Similarly, we have a map

$$
i_{\widetilde{\varpi}=0}^{*} j_{v \neq 0 *}\left(\mathrm{IC}_{\mu} \otimes \overline{\mathbb{Q}}_{\ell}\right)[-1] \rightarrow j_{\bar{k} *}\left(\mathrm{IC}_{\mu, \bar{k}} \otimes \overline{\mathbb{Q}}_{\ell}\right) \text {. }
$$

The key observation, which we will prove later, is that 
Lemma 9.20. The map (9.13) is an isomorphism.

From this, we obtain the following correspondence

$$
{ }^{p} \mathrm{H}^{0} i^{*} j_{\bar{k} *}\left(\mathrm{IC}_{\mu, \bar{k}} \otimes \mathbb{Q}_{\ell}\right) \leftarrow{ }^{p} \mathrm{H}^{0} i^{*} j_{v \neq 0 *}\left(\mathrm{IC}_{\mu} \otimes \overline{\mathbb{Q}}_{\ell}\right)[-1] \rightarrow{ }^{p} \mathrm{H}^{0} i^{*} j_{v=\tilde{\varpi} *} \mathcal{S}_{\tilde{F}}\left(V_{\mu}\right),
$$

with the first arrow an isomorphism. Therefore, we can invert the first arrow of (9.14) and obtain an arrow as in (9.12). To show that this gives an isomorphism, it is enough to show that it induces an isomorphism on cohomology since as we mentioned before, $\mathrm{H}^{*}: \operatorname{Perv}_{L^{+} P_{\bar{k}}}\left(\operatorname{Gr}_{P_{\bar{k}}}, \overline{\mathbb{Q}}_{\ell}\right) \rightarrow \operatorname{Vect}\left(\overline{\mathbb{Q}}_{\ell}\right)$ is faithful.

Let $f: \widetilde{\operatorname{Gr}}_{\mathcal{G}, X} \rightarrow \mathbb{A}_{\mathcal{O}_{\tilde{F}}}^{1}$ be the structure map, which is ind-proper. Therefore the (derived) push-forward $f_{*}$ commutes with any pullback and pushforward. Since over $\left(\mathbb{G}_{m}\right)_{\mathcal{O}_{\tilde{F}}} \subset \mathbb{A}_{\mathcal{O}_{\tilde{F}}}^{1}, f_{*}\left(\mathrm{IC}_{\mu} \otimes \overline{\mathbb{Q}}_{\ell}\right)$ is just constant, with stalks isomorphic to $V_{\mu}[1]$, it is immediately seen that

$$
f_{*} i^{*} j_{\bar{k} *}\left(\mathrm{IC}_{\mu, \bar{k}} \otimes \mathbb{Q}_{\ell}\right) \simeq f_{*} i^{*} j_{v \neq 0 *}\left(\mathrm{IC}_{\mu} \otimes \overline{\mathbb{Q}}_{\ell}\right)[-1] \simeq f_{*} i^{*} j_{v=\tilde{\varpi} *} \mathcal{S}_{\tilde{F}}\left(V_{\mu}\right) \simeq V_{\mu} .
$$

Observe that the triangle (9.11) implies that $i^{*} j_{\bar{k} *}\left(\mathrm{IC}_{\mu, \bar{k}} \otimes \mathbb{Q}_{\ell}\right)={ }^{p} \mathrm{H}^{0}\left(i^{*} j_{\bar{k} *}\left(\mathrm{IC}_{\mu, \bar{k}} \mathbb{Q}_{\ell}\right)\right)+$ ${ }^{p} \mathrm{H}^{1}\left(i^{*} j_{\bar{k} *}\left(\mathrm{IC}_{\mu, \bar{k}} \otimes \mathbb{Q}_{\ell}\right)\right)[-1]$ as objects in $\mathrm{D}_{L^{+} P_{\bar{k}}}\left(\operatorname{Gr}_{P_{\bar{k}}}, \overline{\mathbb{Q}}_{\ell}\right)$ (and similarly for $i^{*} j_{v=\tilde{\omega} *} \mathcal{S}_{\tilde{F}}\left(V_{\mu}\right)$ ). Therefore the isomorphism $\mathrm{H}^{*}\left(i^{*} j_{\bar{k} *}\left(\mathrm{IC}_{\mu, \bar{k}} \mathbb{Q}_{\ell}\right)\right) \simeq \mathrm{H}^{*}\left(i^{*} j_{v=\tilde{w} *} \mathcal{S}_{\tilde{F}}\left(V_{\mu}\right)\right)$ implies that the natural map (9.14) induces the isomorphism

$$
\mathrm{H}^{*}\left({ }^{p} \mathrm{H}^{0} i^{*} j_{\bar{k} *}\left(\mathrm{IC}_{\mu, \bar{k}} \otimes \mathbb{Q}_{\ell}\right)\right) \simeq \mathrm{H}^{*}\left({ }^{p} \mathrm{H}^{0} i^{*} j_{v=\tilde{\varpi} *} \mathcal{S}_{\tilde{F}}\left(V_{\mu}\right)\right)
$$

and the theorem follows.

It remains to prove Lemma 9.20, This will follow if we can show:

Lemma 9.21. Consider the natural structure map $\widetilde{\mathrm{Gr}}_{\mathcal{G}, X} \rightarrow \mathcal{O}_{\tilde{F}}$. Then:

(i) The vanishing cycle $\mathrm{R} \Phi^{\widetilde{\mathrm{Gr}}_{\mathcal{G}, X}}\left(j_{v \neq 0 *}\left(\mathrm{IC}_{\mu} \nabla \overline{\mathbb{Q}}_{\ell}\right)\right)$ is trivial.

(ii) The natural map

$$
\mathrm{R} \Psi^{\widetilde{\mathrm{Gr}}_{\mathcal{G}, X}}\left(j_{\tilde{F} *}\left(\mathrm{IC}_{\mu, \tilde{F}} \nabla \overline{\mathbb{Q}}_{\ell}\right)\right) \rightarrow j_{\bar{k} *} \mathrm{R} \Psi^{\widetilde{\mathrm{Gr}}_{\mathcal{G}, \mathbb{G}_{m}}}\left(\mathrm{IC}_{\mu, \tilde{F}} \otimes \overline{\mathbb{Q}}_{\ell}\right) \simeq j_{\bar{k} *}\left(\mathrm{IC}_{\mu, \bar{k}} \otimes \overline{\mathbb{Q}}_{\ell}\right)
$$

is an isomorphism.

Indeed, the natural map (9.13) factors as

$$
i_{\tilde{\varpi}=0}^{*} j_{v \neq 0 *}\left(\mathrm{IC}_{\mu} \otimes \overline{\mathbb{Q}}_{\ell}\right)[-1] \rightarrow \mathrm{R} \Psi^{\widetilde{\operatorname{Gr}}_{\mathcal{G}, X}}\left(j_{\tilde{F}_{*}}\left(\mathrm{IC}_{\mu, \tilde{F}} \otimes \overline{\mathbb{Q}}_{\ell}\right)\right) \rightarrow j_{\bar{k} *}\left(\mathrm{IC}_{\mu, \bar{k}} \otimes \overline{\mathbb{Q}}_{\ell}\right) .
$$

(To see these two maps coincide, apply the adjunction between $i_{\tilde{\varpi}=0}^{*}$ and $i_{\tilde{\varpi}=0 *}$.) Now by Part (i) of the lemma, the first arrow is an isomorphism, and by Part (ii) of the lemma, the second arrow is an isomorphism. This shows that Lemma 9.21 implies Lemma 9.20.

Finally, we prove Lemma 9.21. This statement can be regarded as a global analogue of Lemma 9.4. Since there is no resolution of $\widetilde{\mathrm{Gr}}_{\mathcal{G}, X}$ satisfying the conditions in [25, $\S 5.2]$, we need a different argument. First, we prove (i). According to Gabber's theorem, since $j_{v \neq 0 *}\left(\mathrm{IC}_{\mu} \otimes \overline{\mathbb{Q}}_{\ell}\right)[1]$ is perverse, $\mathrm{R} \Phi^{\widetilde{\operatorname{Gr}}_{\mathcal{G}, X}}\left(j_{v \neq 0 *}\left(\mathrm{IC}_{\mu} \otimes \overline{\mathbb{Q}}_{\ell}\right)\right)$ is a perverse sheaf. On the other hand, by [25, §5.2],

$$
j_{\bar{k}}^{*} \mathrm{R} \Phi^{\widetilde{\mathrm{Gr}}_{\mathcal{G}, X}}\left(j_{v \neq 0 *}\left(\mathrm{IC}_{\mu} \otimes \overline{\mathbb{Q}}_{\ell}\right)\right)=\mathrm{R} \Phi^{\widetilde{\mathrm{Gr}_{\mathcal{G}, \mathbb{G}_{m}}}}\left(j_{v \neq 0}^{*} j_{v \neq 0 *}\left(\mathrm{IC}_{\mu} \otimes \overline{\mathbb{Q}}_{\ell}\right)\right)=(0) .
$$


Therefore, $\mathrm{R} \Phi^{\widetilde{\operatorname{Gr}}_{\mathcal{G}, X}}\left(j_{v \neq 0 *}\left(\mathrm{IC}_{\mu} \nabla \overline{\mathbb{Q}}_{\ell}\right)\right)$ is a perverse sheaf on $\operatorname{Gr}_{P_{\bar{k}}}$, which is $L^{+} P_{\bar{k}}$-equivariant by the same argument as in Lemma 9.2. To show it is trivial, it is enough to prove that its cohomology vanishes. To do this we can apply $f_{*}$ with $f: \widetilde{\operatorname{Gr}}_{\mathcal{G}, X} \rightarrow \mathbb{A}_{\mathcal{O}_{\tilde{F}}}^{1}$ as above, and we are done.

To prove (ii), as the nearby cycle functor commutes with Verdier duality (cf. [29, Theorem 4.2]), it is enough to prove the dual statement that the natural map

$$
j_{\bar{k} !}\left(\mathrm{IC}_{\mu, \bar{k}} \otimes \overline{\mathbb{Q}}_{\ell}\right) \rightarrow \mathrm{R} \Psi^{\widetilde{\mathrm{Gr}}_{\mathcal{G}, X}} j_{\tilde{F} !}\left(\mathrm{IC}_{\mu, \tilde{F}} \otimes \overline{\mathbb{Q}}_{\ell}\right)
$$

is an isomorphism. But the vanishing cycle $\mathrm{R} \Phi^{\widetilde{G r}_{\mathcal{G}, X}}\left(j_{v \neq 0 !}\left(\mathrm{IC}_{\mu} \otimes \overline{\mathbb{Q}}_{\ell}\right)\right)$ is trivial by a similar argument as above, and therefore

$$
\begin{aligned}
\mathrm{R} \Psi^{\widetilde{\mathrm{Gr}}_{\mathcal{G}}, X} j_{\tilde{F} !}\left(\mathrm{IC}_{\mu, \tilde{F}} \otimes \overline{\mathbb{Q}}_{\ell}\right) & \simeq i_{\tilde{\omega}=0}^{*} j_{v \neq 0 !}\left(\mathrm{IC}_{\mu} \nabla \overline{\mathbb{Q}}_{\ell}\right)[-1] \simeq \\
& \simeq j_{\bar{k} !} i_{\tilde{\omega}=0}^{*}\left(\mathrm{IC}_{\mu} \otimes \overline{\mathbb{Q}}_{\ell}\right)[-1] \simeq j_{\bar{k} !}\left(\mathrm{IC}_{\mu, \bar{k}} \nabla \overline{\mathbb{Q}}_{\ell}\right) .
\end{aligned}
$$

This completes the proof of Lemma 9.21 and the theorem.

Remark 9.22. It is possible that the theory of nearby cycles over a higher dimensional base, see for example [43], could provide an different approach to the above arguments.

Let us use Theorem 9.18 to determine the inertia action on $\mathrm{R} \Psi_{\mu}$. Let $H^{\vee} \rtimes \operatorname{Gal}(\bar{F} / E)$ be the Langlands dual group of $G_{E}$. Then according to [58, $\mathrm{H}^{*}\left(\mathcal{F}_{\mu}\right)$ carries a canonical action of $H^{\vee} \rtimes \operatorname{Gal}(\bar{F} / E)$, that factors through $H^{\vee} \rtimes \operatorname{Gal}(\tilde{F} / E)$, whose underlying representation of $H^{\vee}$ is just $V_{\mu}$ and whose underlying representation of $\operatorname{Gal}(\tilde{F} / E)$ is (a certain twist of) the natural action of $\operatorname{Gal}(\tilde{F} / E)$ on $\mathrm{H}^{*}\left(\mathcal{F}_{\mu}\right)$. In addition, when we restrict this action to $I_{E}$, the twist disappears. Therefore, $V_{\mu}$ is naturally a $\left(H^{\vee}\right) \rtimes I_{E}$-module. Since nearby cycles commute with proper push-forward, we have $\mathrm{H}^{*}\left(\mathrm{R} \Psi_{\mu}\right) \simeq \operatorname{Res}\left(V_{\mu}\right)$ as a representation of $\left(H^{\vee}\right)^{I_{F}} \times I_{E}$. Therefore, we obtain that

Theorem 9.23. Under the isomorphism $\mathcal{S}_{\bar{k}}\left(\operatorname{Res}\left(V_{\mu}\right)\right) \stackrel{\sim}{\rightarrow} \mathrm{R} \Psi_{\mu}$ obtained from Theorem 9.18, the action of the inertia $I_{E}$ on $\mathrm{R} \Psi_{\mu}$ corresponds to the action of $I_{E} \subset\left(H^{\vee}\right)^{I_{F}} \times I_{E}$ on $\operatorname{Res}\left(V_{\mu}\right)$.

Example 9.24. Let us consider the following example, which was discussed in [47] (note that here we use different notation). Let $G=\operatorname{Res}_{K / F} \mathrm{GL}_{n}$ and $\mathcal{P}_{x}=\operatorname{Res}_{\mathcal{O}_{K} / \mathcal{O}_{F}} \mathrm{GL}_{n}$, where $K / F$ is a totally tamely ramified extension of degree $d$. In this case, we have $\mathrm{Gr}_{P_{k}} \simeq \mathrm{Gr}_{\mathrm{GL}_{n}}$, and $\mathrm{Gr}_{\mathcal{G}, \mathcal{O}} \otimes_{\mathcal{O}} \tilde{F} \simeq\left(\mathrm{Gr}_{\mathrm{GL}_{n}}\right)^{d}$. In addition, $H^{\vee}=\mathrm{GL}_{n}^{d}$, and $\left(H^{\vee}\right)^{I}=\mathrm{GL}_{n}$, embedded in $H^{\vee}$ via the diagonal embedding.

Let $\tilde{F}$ be the Galois closure of $K / F$. Choose an ordering of the set of embeddings $\phi: \tilde{F} \rightarrow \bar{F}$ which allows us to identify $\operatorname{Gal}(\tilde{F} / F)$ with a subgroup of the symmetric group $S_{d}$. Let $\mu$ be a minuscule coweight of $G$. We can write $\mu=\left(\mu_{1}, \mu_{2}, \ldots, \mu_{d}\right)$ with $\mu_{i}: \mathbb{G}_{\mathrm{m}} \tilde{F} \rightarrow\left(\mathrm{GL}_{n}\right)_{\tilde{F}}$; if $E$ is the reflex field of $\mu$, the $\operatorname{Galois} \operatorname{group} \operatorname{Gal}(\tilde{F} / E)$ is the subgroup of those $\sigma \in S_{d}$ with $\mu_{\sigma(i)}=\mu_{i}$. In this case,

$$
\operatorname{Res}\left(V_{\mu}\right)=V_{\mu_{1}} \otimes \cdots \otimes V_{\mu_{d}} .
$$


as a representation of $\mathrm{GL}_{n}=\left(H^{\vee}\right)^{I}$. Therefore, Theorem 9.18 gives

$$
\widetilde{\mathrm{R \Psi}}_{\mu} \simeq \mathrm{IC}_{\mu_{1}} \star \mathrm{IC}_{\mu_{2}} \star \cdots \star \mathrm{IC}_{\mu_{d}} .
$$

Indeed, this has been proved in [47, Theorem 7.1]. As the action of $\operatorname{Gal}(\tilde{F} / E)$ on $V_{\mu_{1}} \otimes$ $\cdots \otimes V_{\mu_{d}}$ commutes with the action of $\left(H^{\vee}\right)^{I_{F}}$, we can write

$$
V_{\mu_{1}} \otimes \cdots \otimes V_{\mu_{d}}=\bigoplus_{\lambda \leq \mu_{1}+\cdots+\mu_{d}} M_{\lambda} \otimes V_{\lambda}
$$

where each $M_{\lambda}$ is a representation of $\operatorname{Gal}(\tilde{F} / E)$. Therefore, Theorem 9.23 now implies that

$$
\mathrm{R} \Psi_{\mu} \simeq \bigoplus_{\lambda \leq \mu_{1}+\cdots+\mu_{d}} M_{\lambda} \otimes \mathrm{IC}_{\lambda}
$$

and the action of $\operatorname{Gal}(\tilde{F} / E)$ on $\mathrm{R} \Psi_{\mu}$ is through the action on each $M_{\lambda}$. This decomposition was conjectured in [47, Remark 7.4]. Actually, it seems that our techniques can be extended to obtain this even when $K / F$ is wildly ramified but we prefer to leave this for another time.

9.d.5. Finally, let us determine the (semi-simple) trace $\tau_{\mathrm{R} \Psi_{\mu}}^{\mathrm{SS}}$ in the case that $G$ is quasisplit (but not unramified) and $x$ is very special.

We first recall the following extension of the geometric Satake Langlands isomorphism (cf. [75, Theorem 0.2]). Recall $P_{k}=\mathcal{P}_{x_{k((u))}}$, a parahoric group scheme over $k[[u]]$. The affine flag variety $\operatorname{Gr}_{P_{k}}$ is defined over $k$. Let $\mathfrak{P}_{x}^{0}$ be the category of $L^{+} P_{k}$-equivariant, semi-simple perverse sheaves on $\operatorname{Gr}_{P_{k}}$, pure of weight zero. Then there is an equivalence

$$
\mathcal{S}_{k}: \operatorname{Rep}\left(\left(H^{\vee}\right)^{I} \rtimes \operatorname{Gal}(\bar{k} / k)\right) \stackrel{\sim}{\rightarrow} \mathfrak{P}_{x}^{0},
$$

where we consider $\left(H^{\vee}\right)^{I} \rtimes \operatorname{Gal}(\bar{k} / k)$ as a pro-algebraic group over $\overline{\mathbb{Q}}_{\ell}$ and $\operatorname{Rep}\left(\left(H^{\vee}\right)^{I} \rtimes\right.$ $\operatorname{Gal}(\bar{k} / k))$ is its category of algebraic representations. Let $\bar{\mu} \in\left(\mathbb{X}_{\bullet}(T)_{I}\right)^{\sigma}$ so that the corresponding Schubert variety in $\mathrm{Gr}_{P_{k}}$ is defined over $k$. Let $\mathrm{IC}_{\bar{\mu}}$ be its intersection cohomology sheaf and $A_{\bar{\mu}}$ be the function obtained by taking the Frobenius trace on the stalks of $\mathrm{IC}_{\bar{\mu}}$. Let $W_{\bar{\mu}}=\mathrm{H}^{*}\left(\mathrm{IC}_{\bar{\mu}}\right)$. Via the above geometric Satake isomorphism, $W_{\bar{\mu}}$ is naturally a representation of $\left(H^{\vee}\right)^{I} \rtimes \operatorname{Gal}(\bar{k} / k)$.

Recall that in [75], we call a smooth irreducible representation of $G^{\prime}=\mathcal{G} \times{ }_{X} \operatorname{Spec}(k((u)))$ over $F^{\prime}=k((u))$ to be "unramified" if it has a vector fixed by $P_{k}^{\prime}=\mathcal{P}_{x_{k((u))}}(k[[u]])$. To each such representation, we attach a Langlands parameter

$$
\operatorname{Sat}(\pi): W_{F^{\prime}} \rightarrow{ }^{L} G=H^{\vee} \rtimes \operatorname{Gal}\left(\bar{F}^{\prime s} / F^{\prime}\right),
$$

where $W_{F^{\prime}}$ is the Weil group of $F^{\prime}$, such that $\operatorname{Sat}(\pi)(\gamma)=(1, \gamma)$ for $\gamma \in I_{F^{\prime}}$. Let $\Phi$ be a lift of the Frobenius to $W_{F^{\prime}}$. Then as is shown in loc. cit., up to conjugation, we

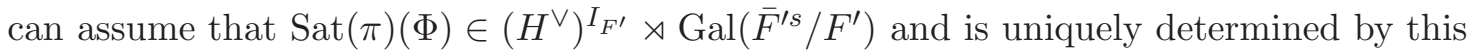

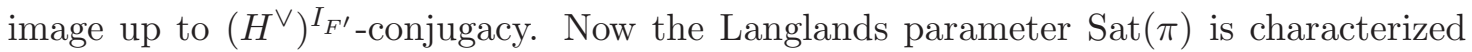
by the following identity.

$$
\operatorname{tr}\left(\pi\left(A_{\bar{\mu}}\right)\right)=\operatorname{tr}\left(\left(\operatorname{Sat}(\pi)(\Phi), W_{\bar{\mu}}\right)\right.
$$


Now, since by Proposition 9.12 the inertia action on $\mathrm{R} \Psi_{\mu}$ factors through a finite quotient, we have

$$
\tau_{\mathrm{R} \Psi_{\mu}}^{\mathrm{sS}}(x)=\operatorname{tr}^{\mathrm{ss}}\left(\sigma_{x},\left(\mathrm{R} \Psi_{\mu}\right)_{\bar{x}}\right)=\operatorname{tr}\left(\sigma_{x},\left(\mathrm{R} \Psi_{\mu}\right)_{\bar{x}}^{I}\right) .
$$

We determine $\mathrm{R} \Psi_{\mu}^{I}$ as a Weil sheaf on $\mathrm{Gr}_{P_{\bar{k}}}$. As nearby cycles commute with proper pushforward, the cohomology $\mathrm{H}^{*}\left(\mathrm{R} \Psi_{\mu}^{I}\right)=\mathrm{H}^{*}\left(\mathcal{F}_{\mu}\right)^{I}$ is pure of weight zero. Therefore, $\mathrm{R} \Psi_{\mu}^{I}$ is just the direct sum of intersection cohomology sheaves on $\mathrm{Gr}_{P_{\bar{k}}}$, equipped with the natural Weil structure. By the above discussion and the results of the previous section, we obtain that $\tau_{R \Psi_{\mu}}^{\mathrm{ss}} \in \mathcal{H}_{q}\left(G^{\prime}, K^{\prime}\right)$ is the unique function such that

$$
\operatorname{tr}\left(\tau_{R \Psi_{\mu}}^{\mathrm{ss}}(\pi)\right)=\operatorname{tr}\left(\operatorname{Sat}(\pi)(\sigma), V_{\mu}^{I}\right) .
$$

This agrees with the prediction of Haines and Kottwitz.

\section{Appendix: Homogeneous spaces}

In this section, we study the representability of certain quotients of group schemes over a two-dimensional base. The results are used in Chapter 5 to show the ind-representability of the global affine Grassmannian $\operatorname{Gr}_{\mathcal{G}, X}$.

10.a. We assume that $A$ is an excellent Noetherian regular ring of Krull dimension 2. If $G, H$ are smooth group schemes over $A$ and $H \hookrightarrow G$ is a closed group scheme immersion then by Artin's theorem ([3]), the fppf quotient $G / H$ is represented by an algebraic space which is separated of finite presentation and in fact smooth over $A$.

Lemma 10.1. Suppose that $G_{1} \hookrightarrow G_{2} \hookrightarrow G_{3}$ are closed group scheme immersions and $G_{1}, G_{2}, G_{3}$ are smooth over $A$. The natural morphism

$$
G_{3} / G_{1} \rightarrow G_{3} / G_{2}
$$

is an fppf fibration with fibers isomorphic to $G_{2} / G_{1}$. Suppose that $G_{2} / G_{1}$ is quasi-affine (affine). If $G_{3} / G_{2}$ is a scheme, then so is $G_{3} / G_{1}$. If in addition $G_{3} / G_{2}$ is quasi-affine (resp. affine), then so is $G_{3} / G_{1}$.

Proof. The first statement follows from the fact that fppf descent is effective for quasiaffine schemes. In fact, by our assumption, $G_{3} / G_{1} \rightarrow G_{3} / G_{2}$ is a quasi-affine morphism. If $G_{3} / G_{2}$ is quasi-affine, then $G_{3} / G_{1}$ is also quasi-affine (affine) since its structure morphism to $A$ is a composition of quasi-affine (resp. affine) morphisms and as such is also quasi-affine (resp. affine).

Proposition 10.2. Let $\mathcal{G}, \mathcal{H} \rightarrow S=\operatorname{Spec}(A)$ be two smooth affine group schemes with connected fibers. Assume that $\mathcal{H}$ is a closed subgroup scheme of $\mathcal{G}$.

$\operatorname{Set} \mathcal{G}=\operatorname{Spec}(B)$, so that $B$ is an $A-H o p f$ algebra. Then there is a free finitely generated $A$-module $M=A^{n}$ with $\mathcal{G}$-action (i.e a $B$-comodule) and a projective $A$-submodule $W \subset$ $M$ which is a locally a direct summand, such that:

i) There is a $\mathcal{G}$-equivariant surjection $\operatorname{Sym}_{A}^{\bullet}(M) \rightarrow B$ and the $\mathcal{G}$-action on $M$ gives a group scheme homomorphism $\rho: \mathcal{G} \hookrightarrow \mathrm{GL}(M)$ which is a closed immersion. 
ii) The representation $\rho$ identifies $\mathcal{H}$ with the subgroup scheme of $\mathcal{G}$ that stabilizes $W$.

Proof. Write $\mathcal{G}=\operatorname{Spec}(B), \mathcal{H}=\operatorname{Spec}\left(B^{\prime}\right)$ and let $p: B \rightarrow B^{\prime}$ be the ring homomorphism that corresponds to $\mathcal{H} \subset \mathcal{G}$. Observe that $B, B^{\prime}$ are Hopf algebras over $A$. We will often refer to the $B$-comodules for the Hopf algebra $B$ as "modules with $\mathcal{G}$-action". Since $\mathcal{G}, \mathcal{H}$ are smooth with connected geometrical fibers both $B$ and $B^{\prime}$ are projective $A$-modules by Raynaud-Gruson [57, Proposition 3.3.1]. We start with two lemmas.

Lemma 10.3. Let $P$ be a projective $A$-module and $N \subset P$ be a finitely generated $A$ submodule. Then the A-torsion submodule of $P / N$ is finitely generated.

Proof. As $P$ is a direct summand of a free $A$-module, we can assume that $P=A^{I}$ itself is free, with a basis $\left\{e_{i} ; i \in I\right\}$. Let $n_{1}, \ldots, n_{t}$ be a set of generators of $N$, and write $n_{i}=\sum a_{i j} e_{j}$. Then $J=\left\{j \in I \mid \exists i\right.$ such that $\left.a_{i j} \neq 0\right\}$ is a finite set and $A^{I} / N=A^{J} / N \oplus A^{I-J}$. The conclusion follows.

Lemma 10.4. Let $P$ be a projective $A$-module and suppose that $N \subset P$ is a finitely generated $A$-submodule. If $P / N$ is torsion free, then $N$ is a projective A-module.

Proof. Observe that $N$ is $A$-torsion free. Consider the double dual $N^{\vee \vee}$. We have $N \subset$ $N^{\vee \vee} \subset P$, and $N^{\vee \vee} / N \subset P / N$ is torsion. Therefore, $N=N^{\vee \vee}$, which is projective by our assumption that $A$ is regular and two-dimensional.

Now let us prove the proposition. Observe first [70, Cor. 3.2] and its proof imply (i) of the proposition (In other words, [70 implies that such a $\mathcal{G}$ is linear, i.e a closed subgroup scheme of $\mathrm{GL}_{n}$.) To obtain the proof of the whole proposition we have to refine this construction from [70] to account for the subgroup scheme $\mathcal{H}$.

Let $V \subset B$ be a finitely generated $A$-submodule with $\mathcal{G}$ action that contains both a set of generators of $I=\operatorname{ker}\left(B \rightarrow B^{\prime}\right)$ and a set of generators of $B$ as an $A$-algebra. Let $p(V)$ be the image of $V$ under $p: B \rightarrow B^{\prime}$. The following diagram is a commutative diagram of modules with $\mathcal{G}$-action

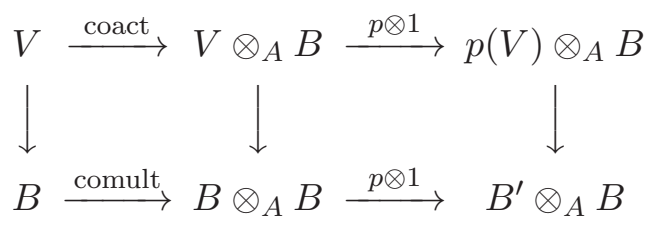

where $\mathcal{G}$ acts on $V \otimes_{A} B, p(V) \otimes_{A} B, B \otimes_{A} B, B^{\prime} \otimes_{A} B$ via the actions on the second factors.

The image of $N:=(p \otimes 1) \cdot \operatorname{coact}(V)$ in $p(V) \otimes_{A} B$ is a finite $A$-submodule with $\mathcal{G}$-action. Let $\epsilon: B \rightarrow A$ be the unit map which splits the natural $A \subset B$. Let $M$ be the image of $N$ under $B^{\prime} \otimes B \stackrel{1 \otimes \epsilon}{\rightarrow} B^{\prime}$. Observe that $M$ is a finite $A$-module, but is not necessarily $\mathcal{G}$-stable. By [62, Prop. 2], we can choose a finite $\mathcal{G}$-stable $A$-module $\tilde{M}$ in $B^{\prime}$ containing $M$. By Lemma 10.3, we can enlarge $\tilde{M}$ if necessary to assume that $B^{\prime} / \tilde{M}$ is torsion free (so $\tilde{M}$ is projective over $A$ by the Lemma 10.4). We regard $\tilde{M}$ as a $\mathcal{G}$-stable submodule of $B^{\prime} \otimes_{A} B$ via $\tilde{M} \subset B^{\prime}=B^{\prime} \otimes_{A} A \subset B^{\prime} \otimes_{A} B$ (this is indeed a $\mathcal{G}$-stable submodule since the inclusion $A \subset B$ is $\mathcal{G}$-equivariant). Let $\tilde{N}=\tilde{M}+N$. Then $\tilde{N}$ is a finite $\mathcal{G}$-stable 
submodule of $B^{\prime} \otimes_{A} B$, and under the map $1 \otimes \epsilon: B^{\prime} \otimes_{A} B \rightarrow B^{\prime},(1 \otimes \epsilon)(\tilde{N})=\tilde{M}$. Observe that the torsion submodule $t\left(\left(B^{\prime} \otimes_{A} B\right) / \tilde{N}\right) \subset\left(B^{\prime} \otimes_{A} B\right) / \tilde{N}$ is a module with $\mathcal{G}$-action and maps to zero under $\left(B^{\prime} \otimes_{A} B\right) / \tilde{N} \stackrel{1 \otimes \epsilon}{\rightarrow} B^{\prime} / \tilde{M}$. Let $\tilde{N}^{\prime}$ be the preimage of $t\left(\left(B^{\prime} \otimes_{A} B\right) / \tilde{N}\right)$ under $B^{\prime} \otimes_{A} B \rightarrow\left(B^{\prime} \otimes_{A} B\right) / \tilde{N}$. From Lemma 10.3, $\tilde{N}^{\prime}$ is finite $\mathcal{G}$-stable $A$-module, and $(1 \otimes \epsilon)\left(\tilde{N}^{\prime}\right)=\tilde{M}$. In addition, $\tilde{N}^{\prime}$ is locally free since $\left(B^{\prime} \otimes_{A} B\right) / \tilde{N}^{\prime}$ is torsion free.

Let $\tilde{V}$ be the $\mathcal{G}$-stable $A$-submodule of $B$ given by the fiber product

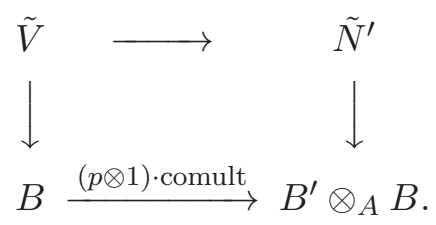

Observe that $(p \otimes 1) \cdot$ comult : $B^{\prime} \rightarrow B^{\prime} \otimes_{A} B$ is injective. Therefore, $\tilde{V}$ is an $A$-submodule of $\tilde{N}^{\prime}$ and therefore it is finitely generated over $A$. In addition, $\tilde{N}^{\prime} \supset N, V \subset \tilde{V}$. Since $B / \tilde{V} \hookrightarrow\left(B^{\prime} \otimes_{A} B\right) / \tilde{N}^{\prime}$ is torsion free, $\tilde{V}$ is projective. Observe that

$$
B \stackrel{(p \otimes 1) \cdot \text { comult }}{\longrightarrow} B^{\prime} \otimes_{A} B \stackrel{1 \otimes \epsilon}{\rightarrow} B^{\prime}
$$

is just the projection $p$. Therefore, $p(\tilde{V})=\tilde{M}$.

Therefore, we obtain the following commutative diagram

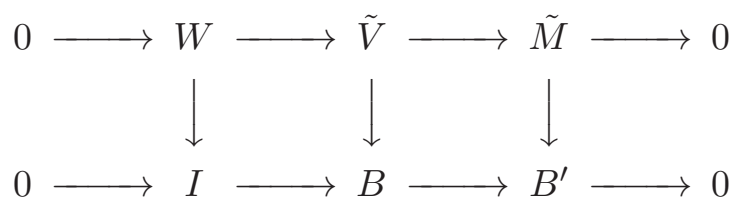

with the first row finitely generated projective $A$-modules. Notice that $\tilde{V} \supset V$ contains a set of generators of the $B$-ideal $I$ and a set of $A$-algebra generators of $B$. Hence, we obtain a closed immersion $\mathcal{G} \rightarrow \operatorname{GL}(\tilde{V})$ of group schemes and we can see that $\mathcal{H}$ can be identified with the closed subgroup scheme of $\mathcal{G}$ that preserves the direct summand $W \subset M:=\tilde{V}$. By replacing $M$ by $M \oplus M^{\prime}$ and $W$ by $W \oplus M^{\prime}$ where $M^{\prime}$ is a finitely generated projective $A$-module with trivial $\mathcal{G}$-action, we can assume that $M$ is $A$-free as desired.

Corollary 10.5. Suppose that $\mathcal{H} \subset \mathcal{G}$ are as in Proposition 10.2. Then the fppf quotient $\mathcal{G} / \mathcal{H}$ is representable by a quasi-projective scheme over $A$.

Proof. By Artin's theorem the fppf quotient $\mathcal{G} / \mathcal{H}$ is represented by an algebraic space over $A$. The algebraic space $\mathcal{G} / \mathcal{H}$ is separated of finite type and even smooth over $A$, the quotient $\mathcal{G} \rightarrow \mathcal{G} / \mathcal{H}$ is also smooth. Take $M$ and $W$ as in Proposition 10.2 and set $P:=\wedge^{\mathrm{rk} W} M$ and $L=\wedge^{\mathrm{rk} W} W \subset \wedge^{\mathrm{rk} W} M=A^{r}$, where $r=\left(\begin{array}{l}\operatorname{rank}(M) \\ \operatorname{rank}(W)\end{array}\right)$. Then, $\mathcal{H}$ is the stabilizer of $[L]$ in $\operatorname{Proj}\left(\wedge^{\mathrm{rk} W} M\right)=\mathbb{P}_{A}^{r-1}$. We obtain a morphism $f: \mathcal{G} \rightarrow \mathbb{P}_{A}^{r-1}$. This gives a monomorphism $\bar{f}: \mathcal{G} / \mathcal{H} \rightarrow \mathbb{P}_{A}^{r-1}$ which is a separated quasi-finite morphism of algebraic spaces. By [33, 6.15], $\mathcal{G} / \mathcal{H}$ is a scheme and we can now apply Zariski's main theorem to $\bar{f}$. We obtain that $\bar{f}$ is a composition of an open immersion with a finite 
morphism and we can conclude that $\mathcal{G} / \mathcal{H}$ is quasi-projective. (See [13, proof of Thm. 2.3.1] for a similar argument).

Remark 10.6. General homogeneous spaces over Dedekind rings are schemes [1, but this is not always the case when the base is a Noetherian regular ring of dimension 2; see [56, X]. In loc. cit. Raynaud asks if $\mathcal{G} / \mathcal{H}$ is a scheme when both $\mathcal{G}$ and $\mathcal{H}$ are smooth and affine over a normal base and $\mathcal{H}$ has connected fibers. The above Corollary gives a partial answer to this question.

Corollary 10.7. Suppose that $\mathcal{G}$ is a smooth affine group scheme with connected fibers over $A$. Then there $n \geq 1$ and a closed subgroup scheme embedding $\mathcal{G} \hookrightarrow \mathrm{GL}_{n}$, such that the fppf quotient $\mathrm{GL}_{n} / \mathcal{G}$ is represented by a smooth quasi-affine scheme over $A$.

Proof. By Proposition 10.2 applied to $\mathcal{G}$ and $\mathcal{H}=\{e\}$, we see that there is a closed subgroup scheme embedding $\rho: \mathcal{G} \hookrightarrow \mathrm{GL}_{m}$ (this follows also directly from [70]). Now apply Corollary 10.5 and its proof to the pair of the group $\mathrm{GL}_{m}$ with its closed subgroup scheme $\mathcal{G}$. We obtain a $\mathrm{GL}_{m}$-representation $\rho^{\prime}: \mathrm{GL}_{m} \rightarrow \mathrm{GL}_{r}=\mathrm{GL}(M)$ that induces a locally closed embedding $\mathrm{GL}_{m} / \mathcal{G} \hookrightarrow \mathbb{P}^{r-1}$. Denote by $\chi: \mathcal{G} \rightarrow \mathbb{G}_{\mathrm{m}}=\operatorname{Aut}_{A}(L)$ the character giving the action of $\mathcal{G}$ on the $A$-line $L$ (as in the proof of Corollary [10.5) and consider $\mathcal{G} \rightarrow \mathrm{GL}_{m} \times \mathbb{G}_{\mathrm{m}}$ given by $g \mapsto\left(\rho^{\prime}(g), \chi^{-1}(g)\right)$. Consider the quotient $\left(\mathrm{GL}_{m} \times \mathbb{G}_{\mathrm{m}}\right) / \mathcal{G}$; to prove it is quasi-affine it is enough to reduce to the case that $A$ is local. Then $L$ is free, $L=A \cdot v$, and $\mathcal{G}$ is the subgroup scheme of $\mathrm{GL}_{m} \times \mathbb{G}_{\mathrm{m}}$ (acting by $\left.(g, a) \cdot m=a \rho^{\prime}(g)(m)\right)$ that fixes $v$. This gives a quasi-finite separated monomorphism $\left(\mathrm{GL}_{m} \times \mathbb{G}_{\mathrm{m}}\right) / \mathcal{G} \rightarrow \mathbb{A}^{r}$ and so by arguing as in the proof of Corollary 10.5 we see that $\left(\mathrm{GL}_{m} \times \mathbb{G}_{\mathrm{m}}\right) / \mathcal{G}$ is quasi-affine. Consider now the standard diagonal block embedding $\mathrm{GL}_{m} \times \mathbb{G}_{\mathrm{m}} \hookrightarrow \mathrm{GL}_{m+1}$. The quotient $\mathrm{GL}_{m+1} /\left(\mathrm{GL}_{m} \times \mathbb{G}_{\mathrm{m}}\right)$ is affine and we can conclude using Lemma 10.1.

\section{REFERENCES}

[1] S. Anantharaman, Schémas en groupes, espaces homogènes et espaces algébriques sur une base de dimension 1, Sur les groupes algébriques, Soc. Math. France, Paris, 1973, pp. 5-79. Bull. Soc. Math. France, Mém. 33.

[2] S. Arkhipov and R. Bezrukavnikov, Perverse sheaves on affine flags and Langlands dual group, Israel J. Math. 170 (2009), 135-183, With an appendix by Bezrukavrikov and Ivan Mirković.

[3] M. Artin, Versal deformations and algebraic stacks, Invent. Math. 27 (1974), 165-189.

[4] A. Beauville and Y. Laszlo, Un lemme de descente, C. R. Acad. Sci. Paris Sér. I Math. 320 (1995), no. 3, 335-340.

[5] A. Beilinson and V. Drinfeld, Quantization of Hitchin's integrable system and Hecke eigensheaves, Preprint, http://www.math.utexas.edu/users/benzvi/BD/hitchin.pdf.

[6] J. Bernstein and V. Lunts, Equivariant sheaves and functors, Lecture Notes in Mathematics, vol. 1578, Springer-Verlag, Berlin, 1994.

[7] F. Bruhat and J. Tits, Groupes réductifs sur un corps local. II. Schémas en groupes. Existence d'une donnée radicielle valuée, Inst. Hautes Études Sci. Publ. Math. (1984), no. 60, 197-376.

[8] _ Schémas en groupes et immeubles des groupes classiques sur un corps local, Bull. Soc. Math. France 112 (1984), no. 2, 259-301.

[9] Groupes algébriques sur un corps local. Chapitre III. Compléments et applications à la cohomologie galoisienne, J. Fac. Sci. Univ. Tokyo Sect. IA Math. 34 (1987), no. 3, 671-698. 
[10] _ Schémas en groupes et immeubles des groupes classiques sur un corps local. II. Groupes unitaires, Bull. Soc. Math. France 115 (1987), no. 2, 141-195.

[11] V. Chernousov, P. Gille, and A. Pianzola, Torsors over the punctured affine line, Amer. J. Math. vol. 134, no. 6., 2012.

[12] J.-L. Colliot-Thélène, Résolutions flasques des groupes linéaires connexes, J. Reine Angew. Math. 618 (2008), 77-133.

[13] B. Conrad, Reductive group schemes, Notes for the SGA3 Summer School, Luminy 2011, http://math.stanford.edu/ ${ }^{\sim}$ conrad/papers/luminysga3.pdf.

[14] P. Deligne, Travaux de Shimura, Séminaire Bourbaki, 23ème année (1970/71), Exp. No. 389, Springer, Berlin, 1971, pp. 123-165. Lecture Notes in Math., Vol. 244.

[15] B. Edixhoven, Néron models and tame ramification, Compositio Math. 81 (1992), no. 3, 291-306.

[16] G. Faltings, Algebraic loop groups and moduli spaces of bundles, J. Eur. Math. Soc. (JEMS) 5 (2003), no. $1,41-68$.

[17] D. Gaitsgory, Construction of central elements in the affine Hecke algebra via nearby cycles, Invent. Math. 144 (2001), no. 2, 253-280.

[18] P. Gille, Torseurs sur la droite affine, Transform. Groups 7 (2002), no. 3, 231-245.

[19] U. Görtz, On the flatness of models of certain Shimura varieties of PEL-type, Math. Ann. 321 (2001), no. $3,689-727$.

[20] _ On the flatness of local models for the symplectic group, Adv. Math. 176 (2003), no. 1, 89-115.

[21] U. Görtz and T. Haines, The Jordan-Hölder series for nearby cycles on some Shimura varieties and affine flag varieties, J. Reine Angew. Math. 609 (2007), 161-213.

[22] T. Haines, Test functions for Shimura varieties: the Drinfeld case, Duke Math. J. 106 (2001), no. 1, 19-40.

[23] - Introduction to Shimura varieties with bad reduction of parahoric type, Harmonic analysis, the trace formula, and Shimura varieties, Clay Math. Proc., vol. 4, Amer. Math. Soc., Providence, RI, 2005, pp. 583-642.

[24] _ The base change fundamental lemma for central elements in parahoric Hecke algebras, Duke Math. J. 149 (2009), no. 3, 569-643.

[25] T. Haines and B. C. Ngô, Nearby cycles for local models of some Shimura varieties, Compositio Math. 133 (2002), no. 2, 117-150.

[26] T. Haines and M. Rapoport, On parahoric subgroups, Appendix to [4], 2008.

[27] T. Haines and S. Rostami, The Satake isomorphism for special maximal parahoric Hecke algebras, Represent. Theory 14 (2010), 264-284.

[28] X. He, Normality and Cohen-Macaulayness of local models of Shimura varieties, Preprint. arXiv:1202.4119, 2012.

[29] L. Illusie, Autour du théorème de monodromie locale, Astérisque (1994), no. 223, 9-57, Périodes p-adiques (Bures-sur-Yvette, 1988).

[30] N. Jacobson, A note on hermitian forms, Bull. Amer. Math. Soc. 46 (1940), 264-268.

[31] M. Kisin and G. Pappas, Integral models for Shimura varieties with parahoric level structure, in preparation.

[32] M. Kneser, Galois-Kohomologie halbeinfacher algebraischer Gruppen über p-adischen Körpern. II, Math. Z. 89 (1965), 250-272.

[33] D. Knutson, Algebraic spaces, Lecture Notes in Mathematics, Vol. 203, Springer-Verlag, Berlin, 1971.

[34] R. Kottwitz, Shimura varieties and twisted orbital integrals, Math. Ann. 269 (1984), no. 3, $287-300$.

[35] _ Stable trace formula: cuspidal tempered terms, Duke Math. J. 51 (1984), no. 3, 611-650.

[36] _ Points on some Shimura varieties over finite fields, J. Amer. Math. Soc. 5 (1992), no. 2, 373-444.

[37] _ Isocrystals with additional structure. II, Compositio Math. 109 (1997), no. 3, 255-339. 
[38] R. Kottwitz and M. Rapoport, Minuscule alcoves for $\mathrm{GL}_{n}$ and $\mathrm{GSp}_{2 n}$, Manuscripta Math. 102 (2000), no. 4, 403-428.

[39] E. Landvogt, A compactification of the Bruhat-Tits building, Lecture Notes in Mathematics, vol. 1619, Springer-Verlag, Berlin, 1996.

[40] _ Some functorial properties of the Bruhat-Tits building, J. Reine Angew. Math. 518 (2000), $213-241$.

[41] M. Larsen, Maximality of Galois actions for compatible systems, Duke Math. J. 80 (1995), no. 3, 601-630.

[42] Y. Laszlo and C. Sorger, The line bundles on the moduli of parabolic G-bundles over curves and their sections, Ann. Sci. École Norm. Sup. (4) 30 (1997), no. 4, 499-525.

[43] G. Laumon, Vanishing cycles over a base of dimension $\geq 1$, Algebraic geometry (Tokyo/Kyoto, 1982), Lecture Notes in Math., vol. 1016, Springer, Berlin, 1983, pp. 143-150.

[44] G. Lusztig, Singularities, character formulas, and a q-analog of weight multiplicities, Analysis and topology on singular spaces, II, III (Luminy, 1981), Astérisque, vol. 101, Soc. Math. France, Paris, 1983, pp. 208-229.

[45] I. Mirković and K. Vilonen, Geometric Langlands duality and representations of algebraic groups over commutative rings, Ann. of Math. (2) 166 (2007), no. 1, 95-143.

[46] G. Pappas, On the arithmetic moduli schemes of PEL Shimura varieties, J. Algebraic Geom. 9 (2000), no. 3, 577-605.

[47] G. Pappas and M. Rapoport, Local models in the ramified case. I. The EL-case, J. Algebraic Geom. 12 (2003), no. 1, 107-145.

[48] _ Local models in the ramified case. II. Splitting models, Duke Math. J. 127 (2005), no. 2, $193-250$.

[49] _ Twisted loop groups and their affine flag varieties, Adv. Math. 219 (2008), no. 1, 118-198, With an appendix by T. Haines and M. Rapoport.

[50] L L L L Lal models in the ramified case. III. Unitary groups, J. Inst. Math. Jussieu 8 (2009), no. 3, $507-564$.

[51] G. Pappas, M. Rapoport, and B. Smithling, Local models of Shimura varieties, I. Geometry and combinatorics., to appear in the "Handbook of Moduli", arXiv:1011.5551.

[52] G. Philippe and A. Pianzola, Torsors, Reductive Group Schemes and Extended Affine Lie Algebras, Preprint. arXiv 1109.3405 (to appear, Memoirs of the AMS).

[53] G. Prasad and J-K. Yu, On finite group actions on reductive groups and buildings, Invent. Math. 147 (2002), no. 3, 545-560.

[54] M. Rapoport, A guide to the reduction modulo p of Shimura varieties, Astérisque (2005), no. 298, 271-318, Automorphic forms. I.

[55] M. Rapoport and Th. Zink, Period spaces for p-divisible groups, Annals of Mathematics Studies, vol. 141, Princeton University Press, Princeton, NJ, 1996.

[56] M. Raynaud, Faisceaux amples sur les schémas en groupes et les espaces homogènes, Lecture Notes in Mathematics, Vol. 119, Springer-Verlag, Berlin, 1970.

[57] M. Raynaud and L. Gruson, Critères de platitude et de projectivité. Techniques de "platification" d'un module, Invent. Math. 13 (1971), 1-89.

[58] T. Richarz and X. Zhu, Appendix to 75].

[59] S. Rostami, Kottwitz's nearby cycles conjecture for a class of unitary Shimura varieties., Preprint. arXiv:1112.0074.

[60] P. Scholze, The Langlands-Kottwitz method and deformation spaces of p-divisible groups, J. Amer. Math. Soc. 26 (2013), no. 1, 227-259.

[61] P. Scholze and S. W. Shin, On the cohomology of compact unitary group Shimura varieties at ramified split places, J. Amer. Math. Soc. 26 (2013), no. 1, 261-294.

[62] J.-P. Serre, Groupes de Grothendieck des schémas en groupes réductifs déployés, Inst. Hautes Études Sci. Publ. Math. (1968), no. 34, 37-52. 
[63] _ Galois Cohomology, fifth ed., Lecture Notes in Mathematics, vol. 5, Springer-Verlag, Berlin, 1994.

[64] C. S. Seshadri, Triviality of vector bundles over the affine space $K^{2}$, Proc. Nat. Acad. Sci. U.S.A. 44 (1958), 456-458.

[65] SGA3, Schémas en groupes. III: Structure des schémas en groupes réductifs, Séminaire de Géométrie Algébrique du Bois Marie 1962/64 (SGA 3). Dirigé par M. Demazure et A. Grothendieck. Lecture Notes in Mathematics, Vol. 153, Springer-Verlag, Berlin, 1962/1964.

[66] SGA7I-II, Groupes de monodromie en géométrie algébrique. I-II, Lecture Notes in Mathematics, Vol. 288, Vol. 340, Springer-Verlag, Berlin, 1972-1973, Séminaire de Géométrie Algébrique du BoisMarie 1967-1969 (SGA 7 I-II), Dirigé par A. Grothendieck (I), P. Deligne and N. Katz (II). Avec la collaboration de M. Raynaud et D. S. Rim.

[67] B. Smithling, Topological flatness of orthogonal local models in the split, even case. I, Math. Ann. 350 (2011), no. 2, 381-416.

[68] T. A. Springer, Linear algebraic groups, second ed., Modern Birkhäuser Classics, Birkhäuser Boston Inc., Boston, MA, 2009.

[69] R. Steinberg, Endomorphisms of linear algebraic groups, Memoirs of the American Mathematical Society, No. 80, American Mathematical Society, Providence, R.I., 1968.

[70] R. W. Thomason, Equivariant resolution, linearization, and Hilbert's fourteenth problem over arbitrary base schemes, Adv. in Math. 65 (1987), no. 1, 16-34.

[71] J. Tits, Reductive groups over local fields, Automorphic forms, representations and $L$-functions (Proc. Sympos. Pure Math., Oregon State Univ., Corvallis, Ore., 1977), Part 1, Proc. Sympos. Pure Math., XXXIII, Amer. Math. Soc., Providence, R.I., 1979, pp. 29-69.

[72] T. Tsukamoto, On the local theory of quaternionic anti-hermitian forms, J. Math. Soc. Japan 13 (1961), 387-400.

[73] W. C. Waterhouse and B. Weisfeiler, One-dimensional affine group schemes, J. Algebra 66 (1980), no. 2, 550-568.

[74] J.-K. Yu, Smooth models associated to concave functions in Bruhat-Tits theory, Preprint, 2002, http://www.math.purdue.edu/ jyu/prep/model.pdf.

[75] X. Zhu, The geometric Satake correspondence for ramified groups, Preprint. arXiv:1107.5762.

[76] _ On the coherence conjecture of Pappas and Rapoport, Preprint. arXiv:1012.5979.

Dept. of Mathematics, Michigan State University, E. Lansing, Mi 48824, USA

E-mail address: pappas@math.msu.edu

Dept. of Mathematics, Northwestern University, Evanston, IL 60208, USA

E-mail address: xinwenz@math.northwestern.edu 